\title{
Instrumentality in the Expanded Field of Music Composition
}

\author{
Kevin William Davis
}

A dissertation presented to the faculty of the University of Virginia in candidacy for the degree of Doctor of Philosophy

Department of Music

University of Virginia

May 2017 
Copyright (C) 2017 by Kevin William Davis. All rights reserved. 


\section{Abstract}

This dissertation develops a materialist musical analysis of instrumentality in music composition. Building on the work of Bruno Latour, Martin Heidegger, James J. Gibson, and others, I develop a theory of musical instrumentality that lays the groundwork for alternative readings of often-marginalized compositional practices. To develop this concept of instrumentality and the materialist musical hermeneutic it implies, I draw from theoretical discourses concerned with objects, tools, equipment, and instruments before proceeding to musical instruments specifically. By presenting the musical instrument as an integral part of the compositional frame, I point towards a materialist theory of music, providing an alternative framework for contemporary practices.

Chapter 1 introduces a basic conceptual framework and summarizes the state of scholarship regarding instrumentality and composition. Chapter 2 examines the emergence of music that foregrounds musical instrumentality, focusing on the high modernist period of $1957-1969$ or "the long sixties." This music is interpreted as embodying the central crisis of subject-object relations in modernism as defined by Latour. I show how instrumentality is in flux during this period, indicative of a problematic relationship while also pointing towards possible resolutions. Chapter 3 analyzes the tools of music and instrumentality from a Heideggerian perspective, focusing on and critiquing his Broken Tool analogy. Chapter 4 considers a specifically musical instrumentality, drawing ideas from ecological psychology and phenomenology, to consider the processes of instrumentalization and reinstrumentalization. Chapter 5 contextualizes my creative work in regards to these ideas. 


\section{Acknowledgments}

I would like to first express my gratitude to Ted Coffey, my dissertation chair. His insights and encouragement throughout this process were vital. I would also like to thank my committee, Matthew Burtner, Karl Hagstrom Miller, and Christa Robbins. Each of them brought important ideas to this dissertation. I am indebted to other members of the UVA faculty who provided feedback, including Judith Shatin, Michael Puri, Luke Dahl, Michelle Kisliuk, Fred Maus, Scott DeVeaux, and Rita Felski. I am also grateful to Kamran Ince, Robert Reigle, and Pieter Snapper and the rest of the faculty and staff of MIAM in Istanbul, who helped me lay the groundwork for this research.

This document grew largely out of a seminar on enactive music by Juraj Kojs. In that seminar, I also got to know my doctoral cohort: Chris Peck, Braxton Sherouse, and Paul Turowski. Throughout my doctoral work, each of them has offered critical perspectives, kept me focused and inspired, and provided invaluable personal support. I am also indebted to Victor Szabo, Bonnie Jones, Brian Hulse, and Peter Bussigel, each of whom introduced me to aspects of philosophy and theory that became integral to this research.

I would also like to thank Ryan Maguire, Jon Bellona, and Kristina Warren for their enthusiasm and willingness to help me work through these ideas. Eli Stine, Travis Thatcher, and Jon contributed technical assistance throughout this research. I am appreciative of Tyler Miller for his detailed proofreading. Lastly, I would like to thank my family - my father Gary and my brother Jeff who have long been supportive of my work and my unconventional path through life. 


\section{Dedication}

For my mother 


\section{Table of Contents}

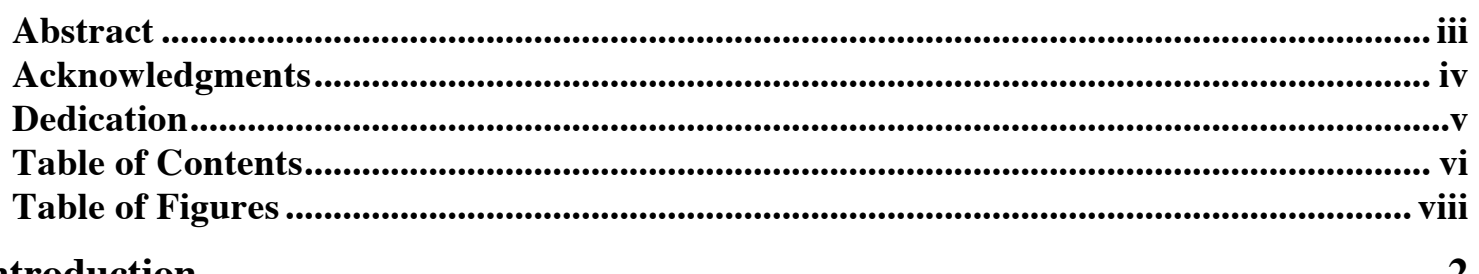

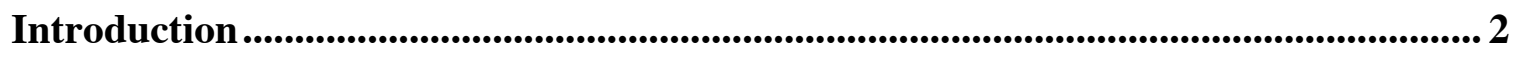

1. The Case for Instrumentality ..............................................................................5 5

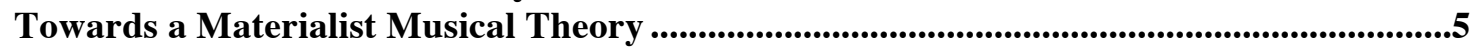

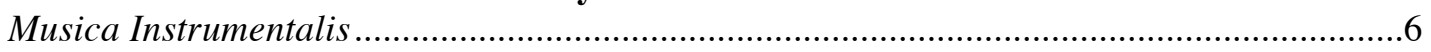

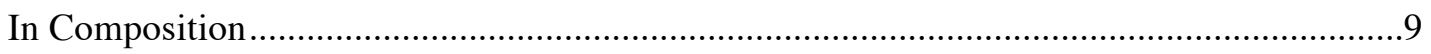

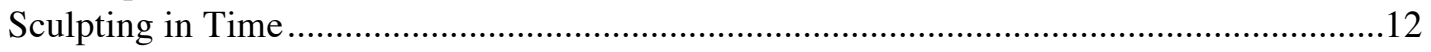

Acts of Composition .......................................................................................................................17

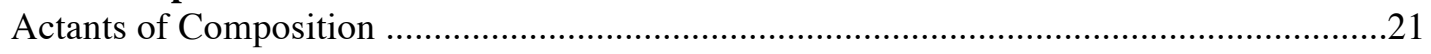

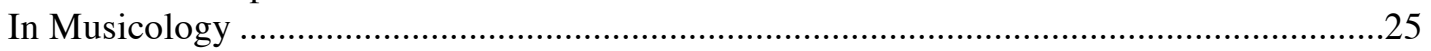

Philosophical Musicology: The Composite Body .............................................................28

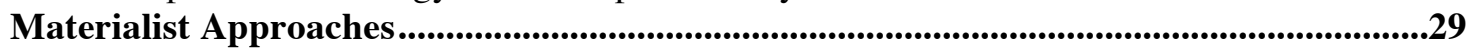

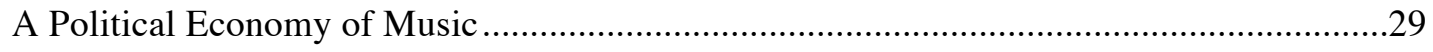

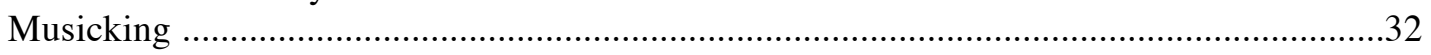

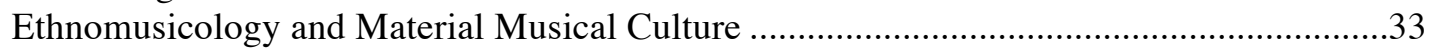

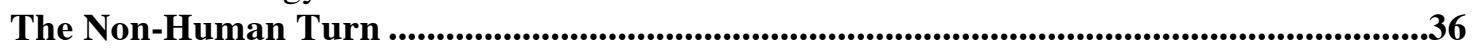

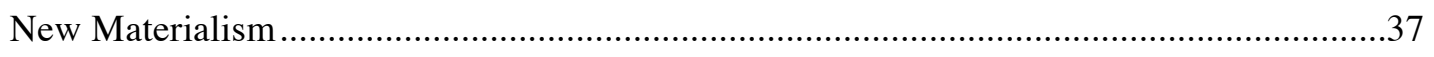

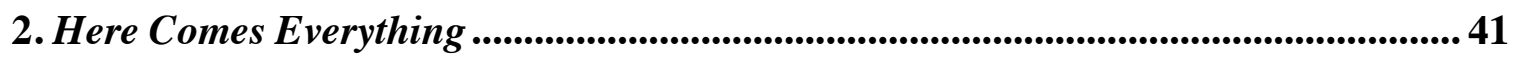

Modernist Aesthetics and the Total Configuration ....................................................................41

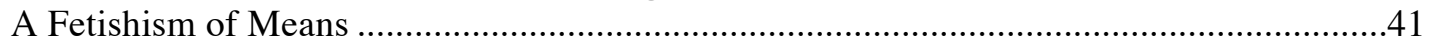

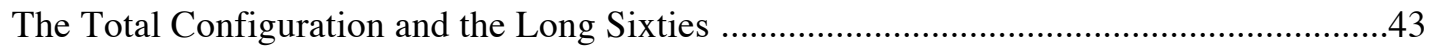

An Age of Wire and String ....................................................................................................45

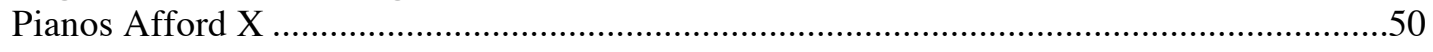

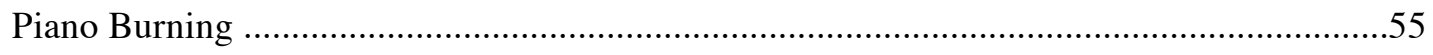

The Isolated Instrument ......................................................................................................59

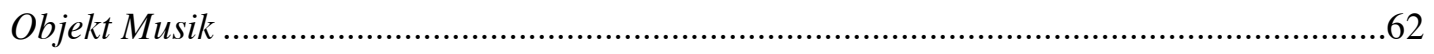

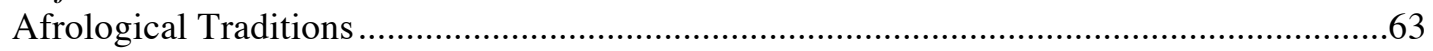

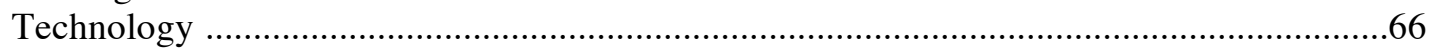

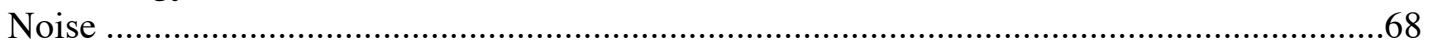

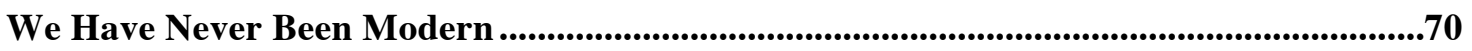

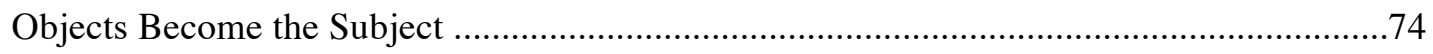

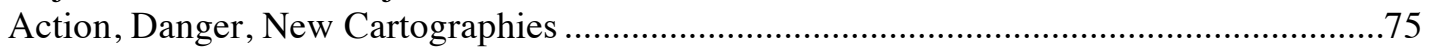

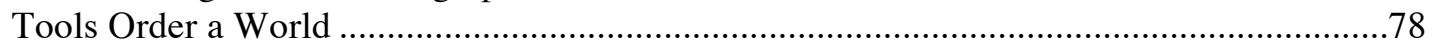

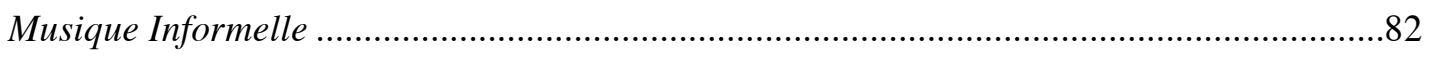

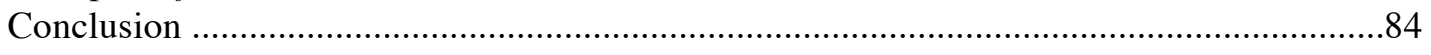

3. The Broken Tool .......................................................................................................... 85

Un-readiness-to-hand ...............................................................................................................85

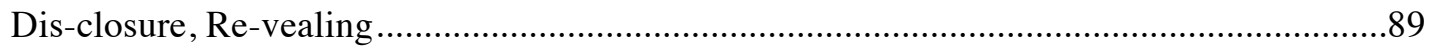




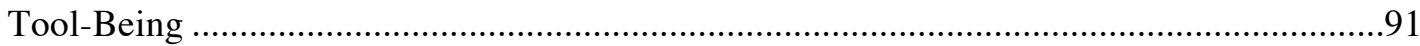

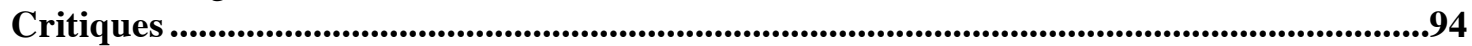

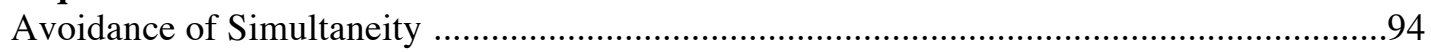

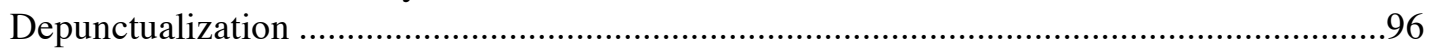

Techne, Technique, Technology; or Technology Reflects Us ..............................................99

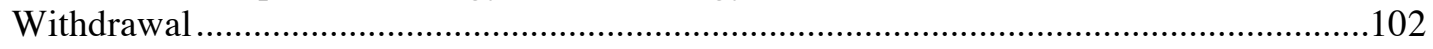

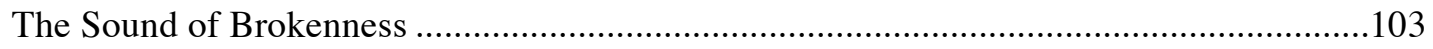

4. Instruments in the Expanded Field ...................................................................... 107

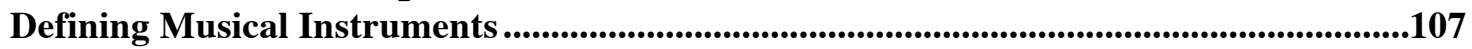

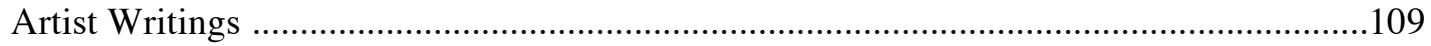

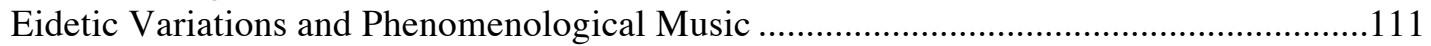

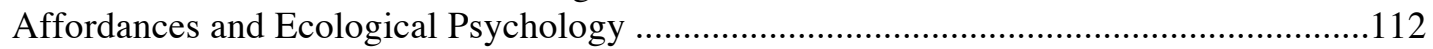

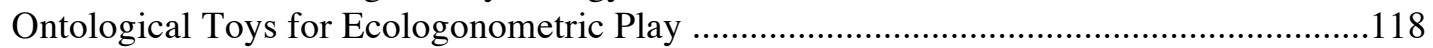

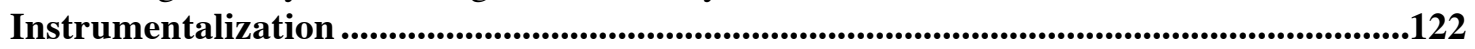

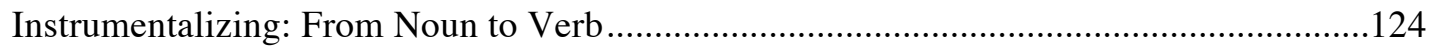

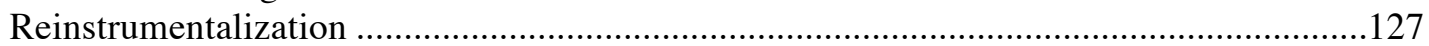

Representations of Reterritorialization .......................................................................................129

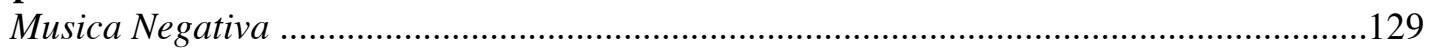

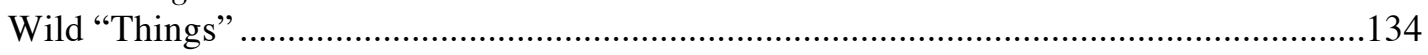

Musical Instrumentality ...........................................................................................................136

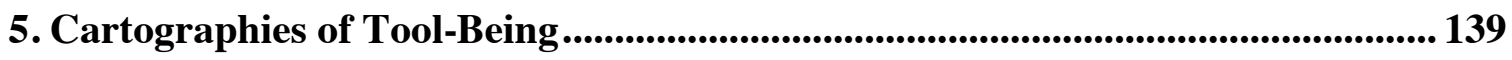

Reinstrumentalization as Composition ...........................................................................................139

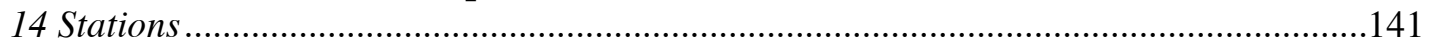

Notated Compositions ...................................................................................................................145

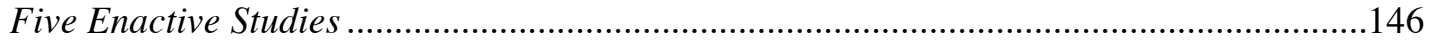

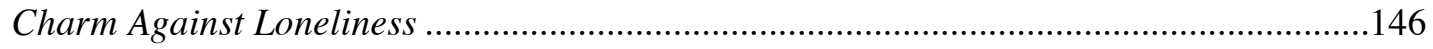

Embracing Mistranslation: Three PFR-3 Poems by Jackson Mac Low ..............................147

Appendices ................................................................................................................................. 152

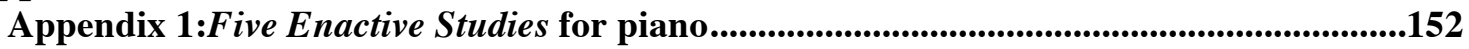

Appendix 2: Charm Against Loneliness .............................................................................................158

Appendix 3: Three PFR-3 Poems by Jackson Mac Low .............................................................183

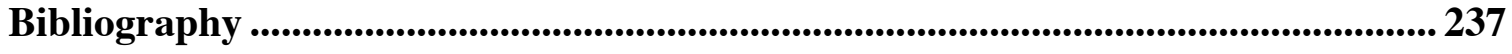




\section{Table of Figures}

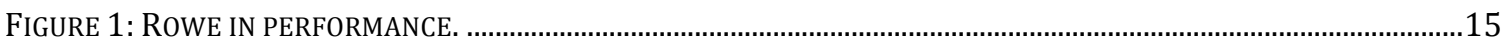

FIGURE 2: TOSHIMARU NAKAMURA AND HIS NO-INPUT MIXING BOARD SETUP. ............................................................16

FIGURE 3: LINEAR FLOW CHART OF CONVENTIONAL MUSICAL MEDIATIONS. .................................................................2

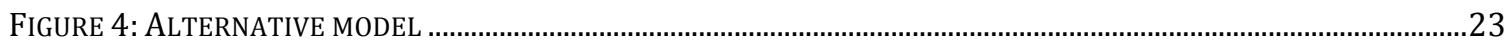

FIGURE 5: EXCERPT FROM TOSHI ICHIYANAGI'S MUSIC FOR PIANO NO. 7 (1961) .........................................................46

FIGURE 6: LABANOTATION EXAMPLE....................................................................................................................4

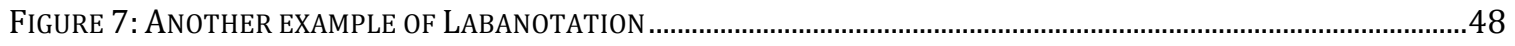

FIGURE 8: EXCERPT FROM STOCKHAUSEN'S KLAVIERSTÜCKE X (1961) ..................................................................50

FIGURE 9: JIMMIE DURANTE IN PERFORMANCE .................................................................................................... 53

Figure 10: OPEnING OF SYlVANo BuSSOTtI's FIVE PIECES FOR DAVID TUDOR, PIECE 4 .............................................54

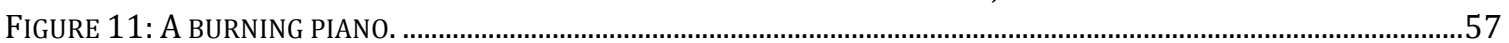

FIGURE 12: JACKSON POLLOCK'S LAVENDER MIST: NUMBER 1, 1950 (1950)...........................................................72

FIGURE 13: EXCERPT FROM CAGE'S CONCERT FOR PIANO AND ORCHESTRA (1960)......................................................76

FIGURE 14: GEORGE MACIUNAS 12 PIANO COMPOSITIONS FOR NAM JUNE PAIK (1960) …….....................................78

FIGURE 15: GORDON MUMMA'S MEDIUM SIZE MOGRAPH 1962 ........................................................................79

FIGURE 16: CORNELIUS CARDEW'S MEMORIES OF YOU (1964) .....................................................................................

FIGURE 17: IMPROVISER BONNIE JONES' NETWORK OF INSTRUMENTALIZED DELAY PEDALS ......................................127

FIGURE 18: JIMI HENDRIX AT THE MONTEREY PoP FESTIVAL ..................................................................................134

FIGURE 19: A PICTURE OF ONE OF THE "STATIONS" ........................................................................................ 142

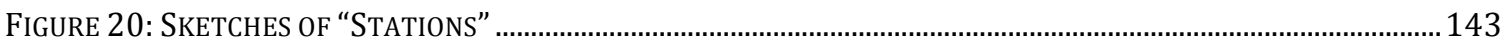

FIGURE 21: PICTURE OF ANOTHER "STATION".................................................................................................. 144

FIGURE 22: AN EARLY GESTURAL REPRESENTATION OF THE SENTENCE “THE PLANETS SHINE” .......................... 149

FIGURE 23: EXAMPLE OF STOPPED HARMONICS IN STANZA 5 FROM FROM “FROM 'THE”” .......................................... 150 
“The world's an untranslatable language without words or parts of speech.

It's a language of objects

Our tongues can't master,

But which we are ardent subjects of."

—Charles Wright, from "The Ghost of Walter Benjamin Walks at Midnight"

"It is therefore not surprising that the soul, having acquired through its nature a body similar to things that set an instrument in motion, which are sinews and breath, is moved with them when they are moved."

-Aristides Quintilianus, On Music

"The object should teach you what it wants to hear."

-David Tudor 


\section{Introduction}

In front of me is an object. It is an object I know very well. Its presence is comfortable, its contours familiar. When seated, part of it rests in contact with my chest, to the left of my sternum; the other part rests on the thumb of my left hand, raised to a height near my chin. I can adjust the balance of this weight effortlessly between chest and hand. I can easily hold up the entire thing with just my left thumb, as it has a metal rod that supports it from the floor. It almost floats.

My left and right knees stabilize the weight asymmetrically, acting unconsciously, gyroscopically. This object that rests against my body effortlessly seems impossibly light; hollow, but just heavy enough to feel substantial. I focus on the curvature of wood just above my right knee. It is an elegant shape, an arc, but one that serves an ergonomic

function: leaving space for the potential movement of the bow, which I hold on the strings with my right hand. The bow feels balanced, despite its odd length. I can sense its tension. It is a circuit. It feels like a part of me. I have to remember to remember it. It is a circuit built to intersect another circuit. I drop my arm and relax. The weight of my arm rests and is transferred through the wood, through the hair, into steel strings, wound tight with just the slightest give.

From here, the cello is a field of relations, a network of correspondences. An object made up of objects, mediated by physics. A thing made of metal, wood, and string, in an equilibrium. It has its own ecology, related to its materials, its design, and the physical world that it inhabits. It also has its own history-including the collective one 
and my own: an economy. An ecologonomy?

The cello is a composite body in a plane of immanence; it is a field of relations: of objects, of forces in flux; of time, of wood, of metal, of tension, of hair, of air, of windings, of friction, of glue, of plastic; of gravity, of thought; of luthiers and designers; of notation, theorists, composers; of audiences.

Consider the bridge: it is a transducer of energy. It must withstand the pressure of the strings (as must the top) while transferring and amplifying energy and not deforming over time. A material and a shape were chosen for this, the height of $16^{\text {th }}$ century technology. There are mercurial sculptural details. Many are unnecessary, indicative of its culture of origin. As a result of its function, design, and decoration, it has a personality, properties of its own; designed with a certain a purpose and functions in mind it also affords new relations, even within its intended context.

When I think of collaboration with the non-human, I think of a sculptor who told me his vocation was decided the day he hit a rock and it "hit him back." I think he meant that in that moment that rock was telling him something, via the chisel and hammerabout itself; about himself; about them, together. Revealing its properties. But when I perform, is the cello the stone? Or is it the hammer? Or is it something altogether different?

Perhaps it is not the difference, but rather the fusion of "subject" and "object" in this scenario that is meaningful. Perhaps the words we use do not adequately describe the complex and subtle ecosystem of musical creation. Maybe the cello is the chisel, or the hammer. Or maybe it is the rock that hits me back. Or perhaps I am the stone being hit, carved by the instrument over time, through the practice of music. Music has always 
been the collaboration between the physical and the imagined, the palpable and the ineffable. It is always a negotiation between the human and the non-human, and instrumentalization its mechanism.

I prepare to play-to act, to make a sound. What I do next embraces the unrepeatable haecceity of this moment. To act here is to participate in the unexpected interactions of objects, forces, and substances within this assemblage. To explore the unbridgeable gulf between the sense of and structure of this object, pointing to towards the inexhaustible nature of music; of sound; of things; of Being. 


\section{The Case for Instrumentality}

“...each instrument, each tool, theoretical or concrete, implies a sound field, a field of knowledge, an imaginable and explorable universe."

- Jacques Attali ${ }^{1}$

\section{Towards a Materialist Musical Theory}

A consideration of musical instrumentality offers an alternative to the dominant narratives of contemporary music and many of its critical and analytical paradigms. Most histories of sound art, improvisation, or other experimental musical practices trace a similar lineage, from Luigi Russolo to John Cage, the often-credited liberators of noise and sound. ${ }^{23}$ Yet sound and noise are resultant phenomena. Both are the result of some physical transfer of energy through a medium or media. Musical sound, in particular, is typically the result of an actor engaging with some instrument. This is true whether it is Sergei Rachmaninoff playing a C\#4 on a grand piano or electromagnetic impulses moving through a speaker coil. The material components of musical sound result from

\footnotetext{
${ }^{1}$ Jacques Attali, Noise: The Political Economy of Music, Theory and History of Literature Volume 16, trans. Brian Massumi (Minneapolis: University of Minnesota Press, 1985), 133.

${ }^{2}$ See Luigi Russolo, The Art of Noise (futurist manifesto), trans. Robert Filliou, Great Bear Pamphlet, Something Else Press, 1967. Accessed February 3, 2017, UbuWeb, http://www.ubu.com/historical/gb/russolo_noise.pdf.

${ }^{3}$ See for instance John Cage, "The Future of Music: Credo," in Silence: Lectures and Writings, (Middletown, CT: Wesleyan University Press, 1973), 3-6.
} 
actions, activities, or processes, which are then translated, transferred, or transformed through or by some instrument, into a medium. This materialist triad can be pictured like this:

$$
\text { Action-Instrument-Sound }{ }^{4}
$$

No musical event can not pass through some version of this performative crucible, no matter what else is occurring, or how displaced or hidden these elements might be, regardless of what expression might be intended or what message is received. It is the prism through which all music is reflected. ${ }^{5}$

For all of the other expressive, historical, cultural, and cognitive elements at play in music making, these materialist elements — despite their multiple manifestations, configurations, and varied distributions - form the phenomenal core of musical events. In materialist terms, this triad is the core of all performed music. This is true despite wide variations in hierarchical stuctures and the inherent malleability of musical assemblages. Though this performative triad exists within a complex contexture, and is capable of elaborate feedbacks, the basic temporal directedness of this process is unavoidable, and the role of the musical instrument itself within it, essential.

\section{Musica Instrumentalis}

Despite the clear importance of these materialist elements, traditional Western notions of

\footnotetext{
${ }^{4}$ The body is of course frequently the link between action and instrument. However, I am trying to build a model that also accommodates electronic music and that also can consider the body and voice as instrumentalized.

${ }^{5}$ Non-cochlear music or imagined scores complicate this matter, but only by subtracting sound from the equation.
} 
music consistently diminish the significance of musical instruments, instrumentality, or indeed many of the materialist or non-human agents within our complex musical ecosystems. This has even included an avoidance, devaluing, or erasure of the body and contributions of the performers themselves. This prejudice against instruments and instrumentalists has its roots in antiquity, in foundational music theorists such as Boethius, Saint Augustine, and Pythagoras. The foundation myths of Western music depend on music being a disembodied phenomenon, ${ }^{6}$ with instrumentalists (instrumentis agitur) occupying the lowest rung of the ladder, a step below composers (fingit carmina), with all of the above being below those able those able to judge music through reason and speculation (instrumentorum opus carmenque diiudicat). Boethius offers a parallel sort quasi-cosmological hierarchy of music overall: Musica mundana - music of the spheres/world, Musica humana - harmony of human body and spiritual harmony, Musica instrumentalis - instrumental music. ${ }^{7}$ Following Pythagoras, the physical manifestation and its vessels were less important than the divine proportions that produced them.$^{8}$ Eventually, there is a kind of medieval erasure of the body in music, which finds its fulfillment in Guido of Arezzo (inventor of solfeggio) through the creation of a theoretical instrument for pure musical contemplation and understanding, another major step towards a minimizing of contact with vibrant, sounding matter. ${ }^{9}$

This avoidance is more problematic than it might seem at first glance. In contemporary scholarship, the dismissal of materialist components can serve to

\footnotetext{
${ }^{6}$ Peter Szendy, Phantom Limbs: On Musical Bodies, (New York: Fordham University Press, 2016), 21-27.

${ }^{7}$ Leslie Blasius, "Mapping the Terrain," in The Cambridge History of Western Music Theory, ed. Thomas Christensen (Cambridge: Cambridge University Press, 2006), 27-38.

${ }^{8}$ Szendy, 2016, 23.

${ }^{9}$ Szendy, 2016, 23.
} 
marginalize certain cultures and promote authoritarian or even racist points-of-view. ${ }^{10}$

Georgina Born, in speaking about the ontology of the musical work makes a point about the musical object that is equally applicable to the instrumental object itself and the performative labor that activates it:

...the embeddedness of the work in broader social relations of class, gender, race and nation, its dependence on patronage or market exchange: none are understood as immanent in the musical object; all are disavowed and denied. ${ }^{11}$

Though Born is speaking in this instance about the musical object in the broadest sense, her point is equally applicable to musical instruments and instrumentality. Born identifies these rigid distinctions between music, musician, and instrument-especially subjectobject relations and human and non-human agencies - as indicative of problematic aspects of both modernism in the broad sense and of much contemporary composition. The ontology of the musical work to Born is dependent upon a hierarchical assemblage and concepts of authorship that diminish the worth of objects, objectifications (recordings), socialization, and performance. ${ }^{12}$ At issue here is not whether there are essential aspects of music that are exclusive of action, instrument, or sound. Nor is the goal of positing instrumentality to diminish the perspectives of artist, listener, or culture. It is instead to recognize how problematic it can be to make a rigid distinction between musical meaning, the physical world, and the tools that make music.

Within music and sound studies, the need for scholarship to accommodate

\footnotetext{
${ }^{10}$ See Georgina Born, “On Musical Mediation: Ontology, Technology and Creativity,” Twentieth-Century Music, 2/1, 28; and George Lewis, "Improvised Music After 1950: Afrological and Eurological Perspectives,' in Audio Culture, eds. Christoph Cox and Daniel Warner (Continuum: New York and London, 2004), 282. Bruno Latour and Timothy Morton even believe that this inability to think the nonhuman has caused, among other things, our current environmental catastrophe. (Bruno Latour, lecture at University of Virginia, 18 September 2016 and Timothy Morton, Hyperobjects: Philosophy and Ecology After the End of the World, (Minneapolis, MN: University of Minnesota Press, 2013).

${ }^{11}$ Born, "On Musical Mediation: Ontology, Technology and Creativity," 28.

${ }^{12}$ Born, "On Musical Mediation: Ontology, Technology and Creativity," 26-27.
} 
instrumentality within composition is more urgent than ever as the corpus of recorded music has grown exponentially, to astronomical proportions, with no end in sight. Technology has allowed instrumentality to subliminally flower into a major component of music of the past century as amplification and recording afford both detailed and archival capturing of the most intricate instrumental actions and interactions. Despite this, there are few new developments in analysis or theory to guide the interested composer, scholar, or critic. This need is especially acute in work in which performance, composition, and production are intertwined (e.g. digitally aided or algorithmic music). Especially urgent for music composition is a conceptual infrastructure that would provide pedagogical and aesthetic paradigms to assist the teaching of composition in a truly contemporary manner. As we in our field grapple with the plurality of contemporary practice, rethinking the nature of instrumentality is especially important to the composition and teaching of contemporary music.

\section{In Composition}

"An open-ness to the totality of sounds implies a tendency away from traditional musical structures towards informality. Governing this tendency - reining it in - are various thoroughly traditional musical structures such as saxophone, piano, violin, guitar, etc., in each of which reposes a portion of the history of music."

- Cornelius Cardew, "Towards an Ethic of Improvisation"13

The topic of instruments and their relationship to music composition specificallyespecially in regards to experimental, indeterminate, or improvised works - is also underexplored. In composition instrumentality is often invisible, to the diminishment of compositional understanding. Though instruments have their own separate discourses

\footnotetext{
${ }^{13}$ Treatise Handbook: Including Bun No. 2, Volo Solo (London, New York: Edition Peters, 1971).
} 
amongst makers, performers, acousticians, orchestrators, and historians, a valuation of instrumentality either theoretically or as compositional material is nascent and within the field of composition, discussions are rare. Musical instruments, like other types of equipment, often withdraw from our view, their effects hidden from us by our very intimacy with them. Rarely are musical instruments acknowledged as musical material in their own right, or still less as important structural features of music. This remains true despite complimentary musicological and historical interventions, such as a focus on the music-making body, sound, or gesture. Much of this scholarship opens the door for materialist analyses while still obscuring their materialist basis.

Many composers and experimental musicians of the Cold War era (1947-1991), particularly in the United States, engaged with the project of exploring the obscured or unused properties of instruments. Often discussed as extended technique, this was as often as not a by-product of experimentation with indeterminacy, notation, performance, electronic music, action, and other experimental methods. There are a multiplicity of highly instrument-oriented and/or object-based practices, beginning in earnest in the late 1950s continuing until the present day: genres like free improvisation, indeterminate composition, action-based music, and installation art, but also Sonorism, music involving movement-based or some highly complex notation systems; jazz, rock, and more experimental forms, like certain noise music or the "American phenomenological school" of composers, like James Tenney, Pauline Oliveros, and Alvin Lucier. ${ }^{14}$

Within the postwar European compositional avant-garde, notated compositions with a focus on experimental notation and extreme instrumental resources was a common

\footnotetext{
${ }^{14}$ This term comes from Tildy Bayar, "Music Inside Out: Spectral Music's Chords of Nature," in the conference proceedings of "Spectral World Musics," eds. Robert Reigle and Paul Whitehead, October 2008,112 .
} 
feature of music from the late 1950s until the early 1970s, and has subsequently returned in waves within certain genres and sub-genres from then until now. In Anglo-American experimentalism of all stripes, from jazz to composition to avant-improvisation and noise music, the material characteristics and sometimes-unpredictable affordances of the instrumental interface have been of paramount importance. Typically going unnoticed in this narrative are African-American musics, in which individual approaches to the instrumental interface and the value of the phenomenal moment were commonplace, and are inseparable from musical structure and meaning..$^{15}$ That these contributions are often underrated and generally ignored in narratives of experimental music makes them no less essential.

These possibilities evolved in parallel to - and in many cases brought on or inspired by — technological advances, such as sound recording and reproduction, amplification, and later sound synthesis. ${ }^{3}$ The latter quarter of the twentieth century saw not only the development of techniques and repertoire for new instruments based on traditional instruments (like the synthesizer, electric guitar, or drum machine), the adaptation of instruments from one culture to another (blues, jazz, rock), but also, in more experimental or institutional practices, of augmented instruments, instrumentalized objects, and the invention of radically new instrumental interfaces. Simultaneously, unprecedented virtuosity, non-traditional notation, and the development of radical alterations or extensions of normative instrumental technique, both on the part of composers and performers, continued apace throughout the century, with unconventional use of conventional instruments becoming a paradoxically commonplace facet of many

\footnotetext{
${ }^{15}$ Lewis, “Improvised Music After 1950: Afrological and Eurological Perspectives," 273-274.
} 
contemporary practices. There are now many composers, improvisers, instrument makers, and programmers whose work consists in large part of exploring and/or interrogating instrumentality.

In many experimental and avant-garde musics, the material character of the instrument and the resultant sounds of such instrumental explorations have themselves become essential components, or indeed in some cases, the music's primary raison d'être. Such instrument-centered or object-based work foregrounds the transformation of action or process - translated through an instrument, an instrumentalized object, or assemblage of objects - into sound. This approach exists in various forms not only in avant-garde circles but also in popular, commercial, and academic environments alike, in both notated and non-notated practices. This is especially significant for traditional musical instruments, as their context with each of these developments is gradually redefined, giving their continued usage new shades of meaning, while its contexture - the networks in which such instruments can interact - also expands. In such music, instrumental exploration is often inseparable from structure. In some cases, it quite literally is structure.

\section{Sculpting in Time ${ }^{16}$}

Consider for instance the music of guitarist and improviser Keith Rowe. Emerging out of the AMM in England in the mid-sixties, Rowe is considered a pioneer in free improvisation and the inventor of tabletop guitar. Working in collaboration with musicians like Eddie Prevost and Cornelius Cardew, Rowe developed a distinctive

\footnotetext{
${ }^{16}$ This section title borrowed from Andrei Tarkovsky, Sculpting in Time: Reflections on the Cinema (New York: Alfred A. Knopf, 1987).
} 
practice of prepared guitar. Rowe coaxes sounds out of the instrument with a combination of traditional and non-traditional objects and implements. This includes things like bows, screws, springs, mechanical devices such as fans, but also devices like a radio, which interact with the magnetic pickups and the electromagnetic environment. It is both sonic and visual, a result of unexpected juxtapositions.

Rowe states that it was painting that inspired his approach to music. He specifically mentions American abstract expressionist painter Jackson Pollock, stating that in order to create a truly American type of painting, Pollock had to change the physical orientation of the canvas by putting it on the floor, thereby divorcing himself from European painting in one fell swoop. ${ }^{17}$ Rowe also speaks of creating a gap between oneself and the instrument, literally and figuratively, by getting the guitar away from his stomach and on to the table. This simultaneously foregrounds the inherent materialist, mechanical aspects of the instrument - but also creates a less expressive, more reflective space, focused less on sentiment and more on phenomena: a pointing away from the self, and instead outwards towards the world..$^{18}$ Similarly to Pollock, this shift in materials necessitated a shift in the very nature of what composition means. For Pollock, a floor-based drip painting was a type of psychic seismograph, the composition of which still had to be contained and composed. In such work a premium is placed on different elements and their own logics - like color, shape, viscosity, density, and so on. Similarly for Rowe there are instrumental, physical, and procedural logics that creates the sounds.

So what does composition mean, if anything, in this context? And how do we

\footnotetext{
17 "Keith Rowe - Prepared Guitar," YouTube video, 9:36, posted by "evilpaul," July 12, 2006, https:/www .youtube.com/watch?v=HnUVpiFHhmM. See also "Keith Rowe," interview with Keith Rowe, Paris Transatlanic, accessed January 27, 2017, http://www.paristransatlantic.com/magazine/interviews/rowe.html.

18 "Keith Rowe - Prepared Guitar," 2006.
} 
account for the role the guitar plays? Improvisations, however sophisticated, are rarely interpreted as a compositional activity despite claims from improvisers regarding the framing of some of their work as explicitly compositional. ${ }^{19}$ Rowe's entire musical practice takes place physically within the field of the electric guitar. In this sense, the guitar itself is inseparable from the composition. It forms the entire frame of the work. The physical structure and design logics define the resultant sound world.

The instrument here plays a central role in the musical structure. ${ }^{20}$ The lack of imposition of a pre-existing compositional syntax or scripted musical architecture allows and encourages enactive processes to become phenomenally heard. Composition here operates from the other side of the fabric: not making the image and coloring in the lines, but corralling and compiling the lines and shapes through actions in time, creating an emergent structure. ${ }^{21}$ This is no less "compositional" than any other music. However the positioning of composition, and therefore of the instrumental object in relation to the music's sounding result is significantly different. In such music, the presence of the instrument, and the very nature of musical instrumentality, becomes more and more important to understanding the work. As Christian Wolff said about saxophonist Evan Parker's music, this is music that is "grounded in the instrument's physical, acoustic construction." 22

\footnotetext{
${ }^{19}$ See "Evan Parker," in The Ashgate Research Companion to Experimental Music, ed. James Sunders (Surrey, England and Burlington, VT: Ashgate, 2009), 335.

${ }^{20}$ Instruments in these kinds of situations have been called a type of inherent score, though this terminology is problematic. See Enrique Thomás, "Musical Instruments as Scores: A Hybrid Approach," in Second Annual Conference for Technologies for Music Notation and Representation (TENOR 2016, Anglia Ruskin University, Cambridge, UK: Anglia Ruskin University, 2016), http://tenor2016.tenorconference.org/papers/16_Tomas_tenor2016.pdf.

${ }^{21}$ Nicholas Cook, Music, Imagination, and Culture (Oxford, New York: Clarendon Press, 1990), 122.

${ }^{22}$ Christian Wolff, Cues: Writings \& Conversations = Hinweise: Schriften und Gespräche, (Köln: MusikTexte, 1998), 404.
} 


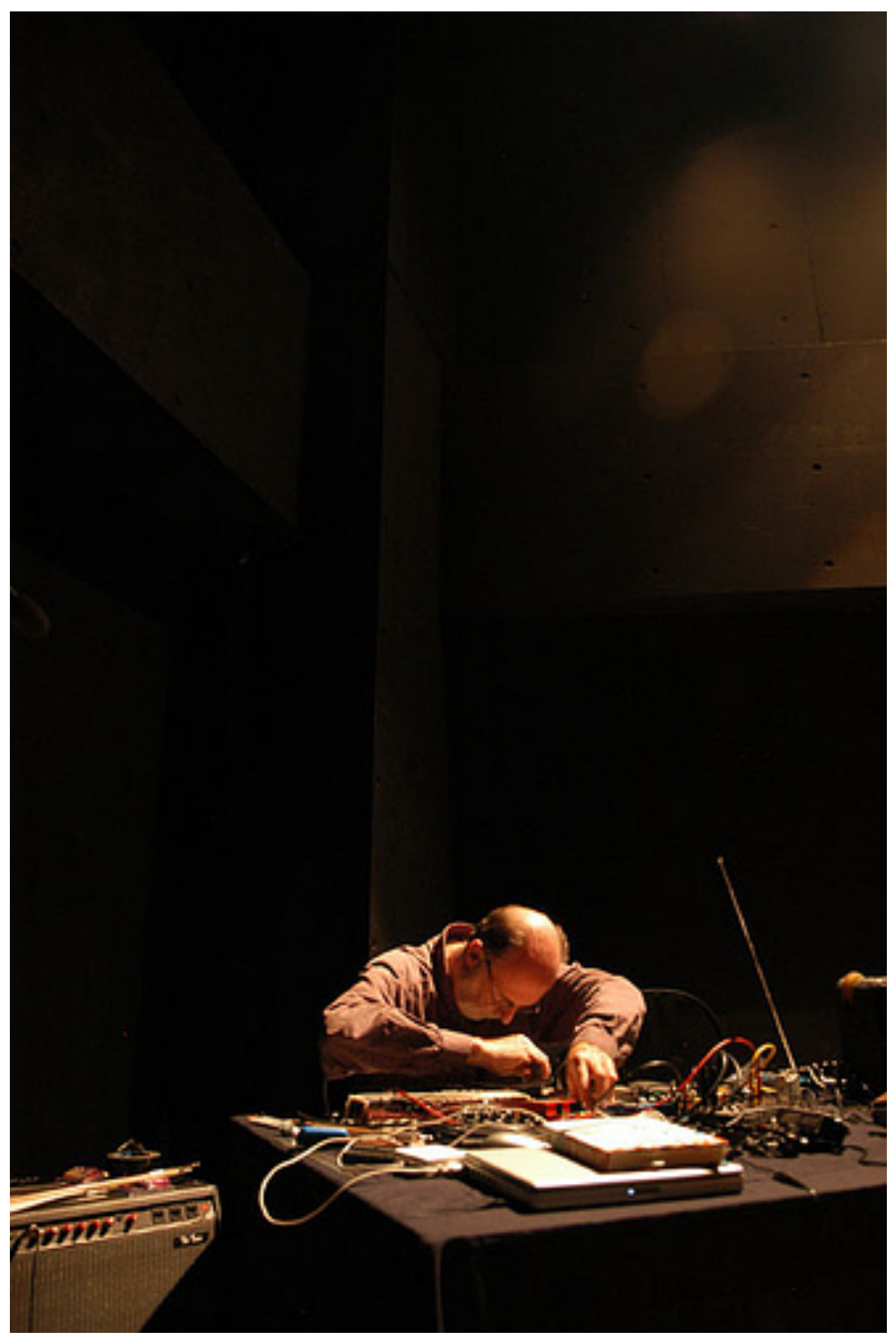

FIGURE 1: ROWE IN PERFORMANCE. 23

A more recent example of this would be the no-input mixing board music of Toshimaru

Nakamura, and entire musical practice based on feedback. In this case the technique has

${ }^{23}$ Downloaded from https://upload.wikimedia.org/wikipedia/commons/a/ad/Rowe_Solo.jpg in March 2017. 
transcended the instrument and travelled to another medium, the humble mixer - an industrially produced device originally used to conglomerate and amplify, is now instrumentalized. Here we are far beyond the realm of extended technique. We are closer to what can be thought of as an instrumentalization, an engagement with the hidden musical properties of a mixer. Nakamura's field of activity is linked to this instrument; it is an exploration of the previously hidden musical properties of this instrumentalized piece of equipment through iterative layers of feedback. Recognizing instrumentality in this scenario is essential to establishing the meaning and value that this music possesses.

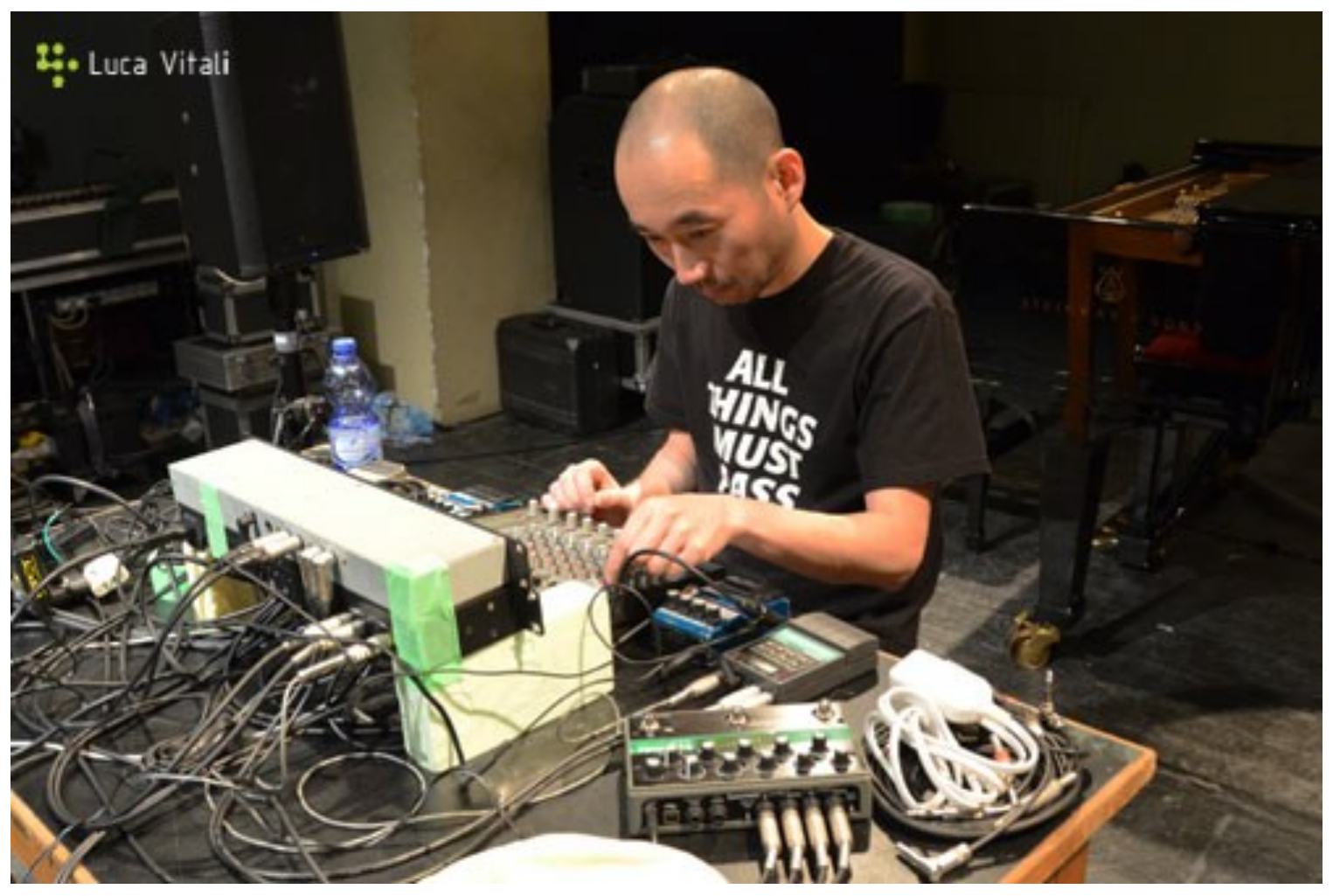

FIGURE 2: TOSHIMARU NAKAMURA AND HIS NO-INPUT MIXING BOARD SETUP. ${ }^{24}$

\footnotetext{
${ }^{24}$ Image downloaded from http://modisti.com/13/wp-content/uploads/2013/06/nakamura.jpg March 2016.
} 


\section{Acts of Composition}

In Music and Discourse, Jean-Jacques Nattiez states that the musical work is not merely a text or even a whole composed of structures (the implicit approaches of musicology and music theory respectively) but rather a musical work is "constituted by the procedures that have engendered it (acts of composition), and the procedures to which it gives rise: acts of interpretation and perception." 25 This powerful thesis gives us a framework for how we might think about music and its composition differently. Nattiez contends that no single perspective can give us a full understanding of a musical work. Accordingly, he develops a tripartite semiotic scheme of musical conceptualization - neutral/immanent, poietic, and esthesic. ${ }^{26}$ In essence, the immanent level is the level of sonic fact such as it can be detected. It is also the purely structural level, insofar as we are capable of objectively accessing musical facticity. The poietic is the level of authorial intent and or action, such as it exists - individual, distributed, or otherwise. The esthetic is the level of interpretation or understanding, within the perceptual or cultural domain. These divisions, far from separating the work into different boxes, gives us a better understanding of the nuances of each component while also sketching a picture of how these different levels interact with each other.

Nattiez claims that all acts of composition are poietic. ${ }^{27}$ But the wide range of acts that can constitute composition, even within a conventional framework, complicates this matter. (In fact, I suspect they are more wide ranging than Nattiez intended.) For

\footnotetext{
${ }^{25}$ Jean-Jacques Nattiez, Music and Discourse: Toward a Semiology of Music, trans. Carolyn Abbate (Princeton, NJ: Princeton University Press, 1990), ix.

${ }^{26}$ Nattiez, 1990, 16-18.

${ }^{27}$ Nattiez, 1990, 92.
} 
instance, later on in Music and Discourse, in an attempt to grapple with acousmatic music within this tripartite scheme, Nattiez elaborates further on his concept of the poetic. He writes: 'By 'poetic' I understand describing the link among the composer's intentions, his creative procedures, his mental schemas, and the result of this collections of strategies; that is, the components that go into the work's material embodiment." ${ }^{28}$ Such a question is intimately related with the question of just what a musical work is, fruitful terrain for many scholars. ${ }^{29}$ Of specific interest for our current purposes is the idea that a musical work includes "all procedures that engendered it" and the labeling of all such procedures "acts of composition." Though in general there remain some underlying assumptions that a composition is something that is ontologically separate from its ultimate structuresomething that precedes and is separate from its immanent manifestation ("A composition" as opposed to "composition") —Nattiez's scheme opens the door for a theoretical understanding of more ambiguous musical activities, which might involve distributed authorship or the explicit compositional presence of other materialist components, like instruments, notation, or space.

Sculpture has dealt with a similar problem. The identity of sculpture in the face of multiple, rapidly expanding practices in the late 1970s (particularly sculpture's forays into architecture and land art) caused confusion to what sculpture actually was. ${ }^{30}$ Postwar sculpture was at that time dealing directly with a parallel problem to the expanded field of music, namely the profusion of materials and the theoretical possibility of any of them

\footnotetext{
${ }^{28}$ Nattiez, 1990, 92.

${ }^{29}$ See for instance Lydia Goehr, The Imaginary Museum of Musical Works: An Essay in the Philosophy of Music, (Oxford: Clarendon Press, 1992); Nicholas Cook, Music, Imagination, and Culture, (Oxford, New York: Clarendon Press, 1990); and Roman Ingarden, The Work of Music and the Problem of Its Identity, trans. Adam Czerniawski, ed. Jean G. Harrell, (Berkeley: University of California Press, 1986).

${ }^{30}$ Rosalind Krauss, "Sculpture in the Expanded Field," in October Volume 8, Spring, 1979, 33.
} 
being subsumed into the discipline.$^{31}$ However, most sculpture is interpreted as a manifestation of some material condition, however complex. As a result, the ground for consideration of sculptural work is intimately involved with materials and readings of cause and effect (through foreground or obfuscation), which are often ignored in music. The long-standing discussions and debates within visual art regarding medium specificity are proof of this. These ideas make sculpture's processes and the significance of objecthood more significant in the sculpture's interpretation. The making of a sculpture is often thought of as a negotiation with the non-human, whether directly (as in stone carving, welding, or throwing lead) or indirectly (as in land art, Cristo's running fence, quasi-architectural practices, or even performance). By contrast, music's relationship to materiality is historically problematic. Compare the debates around medium specificity with music's inability to find a consensus position on the definitions of "musical objects" or even instruments themselves for that matter..$^{32}$ The ephemerality of sound and the amorphous nature of musical objects or the objet sonore of Pierre Schaeffer has proven problematic for musicians, composers, and scholars alike. ${ }^{33}$ In addition, music has the further inherent problems of performativity, means of distribution, reproducibility, semiology, semiography, and more. Material objects and their effects are is less clear, though surely just as present. Perhaps for this reason, sculptural pedagogy and criticism

\footnotetext{
${ }^{31}$ I am using this term in a similar fashion to Chris Peck, not to refer to Krauss' taxonomy of late 1970s sculpture that had expanded to landscape and architecture, but to more refer more broadly to the extreme plurality of modalities of music making. See Chris Peck, "Composition in the Expanded Field of Performance: Experimental Music in Collaboration with Contemporary Dance" (PhD dissertation, University of Virginia, April 2017).

${ }^{32}$ See Mandy Suzanne Wong, "Sound Objects: Speculative Perspectives" (PhD dissertation, University of California Los Angeles, 2012) for the problematic history of the sound object. See also Chapter 4 of this dissertation for examples of instrument definitions.

${ }^{33}$ Antoine Hennion, The Passion for Music: A Sociology of Mediation, (London, New York: Routledge, 2016), 1-13; Pierre Schaeffer, In Search of a Concrete Music, trans. Christine North and John Dack (Berkeley and Los Angeles: University of California Press, 2012), 131-146; and Wong, "Sound Objects," 5.
} 
are more advanced than music in regards to considering and theorizing its relationship to its materials and to materiality. ${ }^{34}$

Unfortunately, in music composition, the questions posed within the discipline in response to the encroachment of this expanded field have been less successful than in visual art. In a similar way to Krauss' overriding interior logic of the monument within sculpture, music has an overriding interior logic of the work. Yet the borders of what constitutes a compositional work have proven to be far more limited than visual art within composition. Certain types of authorship are privileged. The result of this has been a retention of outdated ideas regarding what is and what is not a musical work, which serves to further obscure the materialist elements. The traditional Western prioritizing of the notatable in art music only compounds this problem. Any technique or activity off the beaten path of Western Art Music renders notation representationally problematic. Most unconventional techniques or practices involving instrumentalized objects are therefore often described as "extended" or just plain irrelevant. This has the effect of obscuring the compositional nature of many now longstanding creative practices in multiple genres. ${ }^{35}$

\footnotetext{
${ }^{34}$ Peter Ablinger on noise in music: "As is so often the case, the visual arts discourse on the matter is decades ahead of the music discussion." Peter Ablinger, "Black Square and Bottle Rack," in Noise in and as Music, eds. Aaron Cassidy and Aaron Einbond, (Huddersfield: Huddersfield University Press, 2013), 58. See also Antoine Hennion, "The Cultural Study of Music: A Critical Introduction," in Music and Mediation: Towards a New Sociology of Music (London: Routledge, 2003), 82.

${ }^{35}$ For instance, David Tudor's circuit designs and interactive performative environments simultaneously constitute both instrument design and a composer's practice. Nevertheless the locale of "composition" in this framework remains institutionally and critically problematic. Tudor might be regarded one of the first explicit theorists of the possibilities of musical object in the compositional frame. In Rainforest I-IV (19661973) for instance, Tudor articulated a desire to give loudspeakers the freedom to discover a voice for themselves (see This idea of setting objects to free to make their sounds forms an interesting corollary to the Cageian injunction to let sounds be themselves. A related and very explicit example of this would be Alvin Lucier's Music On A Long Thin Wire (1977) and Opera for Objects (2003) two of many pieces that explore the properties of instrumentalized objects.
} 


\section{Actants of Composition}

It is easy to think of musical instruments as merely a medium or as passive intermediaries. Typically, instruments are thought of as an extension of the body or, more broadly, as a kind of filter of action. ${ }^{36}$ However, I take as axiomatic in this dissertation that musical instruments are not transparent intermediaries, but function as significant mediators of action. Moreover, a critical accounting of instruments and instrumentality is crucial to a full understanding of many experimental and avant-garde practices.

A useful concept in the contextualization of musical instruments and the materialist elements of music overall is the concept of actants. An actant is a term from Bruno Latour's actor-network theory that simply refers anything that acts or shifts actions ${ }^{37}$ This includes things, objects, materials, or forces. Actants are any elements of a network or assemblage that have some effect, regardless of "agency" in the traditional, subject-oriented sense. Essentially, the term actant is a way to avoid the idea of prematurely diminishing the contribution of elements of a network or assemblage through an avoidance of anthropocentric intentionality involved in the word actor. By contrast, an actor is an actant endowed with character, basically a special type of actant. ${ }^{38}$ This way, the idea of explicitly anthropocentric intentionality involved in the word actor can be avoided. Actant subsumes actor. Simultaneously actant allows the idea of instruments, equipment, and objects as social or possessing qualities that affect the assemblage.

The point of this is not to suggest an "absurd symmetry" between humans and

\footnotetext{
${ }^{36}$ See Curt Sachs, The History of Musical Instruments, (New York: W. W. Norton and Company, 1940). See also Tellif Kvite, "What is a Musical Instrument?' Svensk tidskrift för musikforskning, Volume 90, Issue 1, 2008, 45-46.

${ }^{37}$ Madeleine Akrich and Bruno Latour, "A Summary of a Convenient Vocabulary for the Semiotics of Human and Nonhuman Assemblies," in Shaping Technology/Building Society (Cambridge, MA and London: 1992), 259. Though this article is co-authored, the terminology is clearly Latour's.

${ }^{38}$ Akrich and Latour, "A Summary of Convenient Vocabluary," 259.
} 
non-humans, but not to impose a priori some spurious asymmetry among human intentional action and the material world of causal relations.$^{39}$ Imagining actants does not diminish the social, but in fact can extend the concept of what the social can be. ${ }^{40}$ To think of musical instruments as actants forces us to reimagine the human and non-human as engaged in a network of complex, non-linear inter-mediations, in which subject and object are not so clearly differentiated.${ }^{41}$ Such a model allows a dehierarchicalization of relations to more clearly see both the complexity of the social and the interrelatedness of the non-human.

Music itself in this context as can even be reimagined as a network of human and non-human mediators. Sociologist of music Antoine Hennion, for instance, rethinks art as defined by its mediations:

Mediations are neither mere carriers of the work, nor substitutes which dissolve its reality; they are the art itself, as is particularly obvious in the case of music: when the performer places a score on his music stand, he plays that music, to be sure, but music is just as much the very fact of playing; mediations in music have a pragmatic status - they are the art which they reveal and cannot be distinguished from the appreciation they generate..$^{42}$

Hennion claims music can be thought of gaining its very meaning through its complex series of mediations. The mediations are the art itself. If we think of music as collections or assemblages of human and non-human elements in a field of mediation, then the role of the musical instrument seems important to account for. In musical performance,

\footnotetext{
${ }^{39}$ Bruno Latour, Reassembling the Social (Oxford, New York: Oxford University Press), 76.

${ }^{40}$ To paraphrase Latour's analysis of seatbelts, a seatbelt is not an object: it is thousands of people and institutions trying to keep a driver from harm (see Bruno Latour, "Where are the Missing Masses? The Sociology of a Few Mundane Artifacts," in Shaping Technology/Building Society (Cambridge, MA and London: 1992). 225-258.

${ }^{41}$ In the words of Latour, ANT is a theory less about how to study things, but rather "how not to study them - or rather, how to let the actors have some room to express themselves." See Latour, Reassembling the Social, 142.

${ }^{42}$ Hennion, A Passion for Music, 7.
} 
instruments are actants with their own substantial, powerful effects and meanings.

Musical instruments in this abstract sense are transformers of action within an assemblage of the material and non-material, an actant among other actants and actors that translate action into sound.

One way to picture the materialist triad within a network of actants would be to imagine a type of materialist semiological model. A musical arrangement such as the traditional composer-performer-listener model can be seen below:

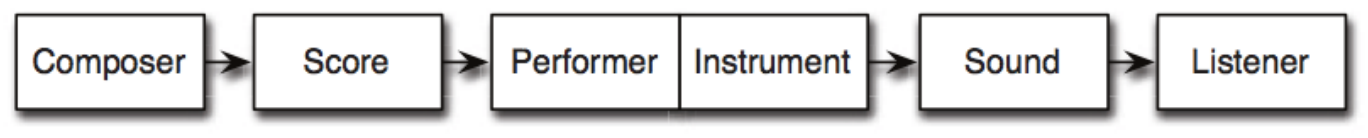

FIGURE 3: LINEAR FLOW CHART OF CONVENTIONAL MUSICAL MEDIATIONS. ${ }^{43}$

And an alternative model that accommodates non-score-based or improvised music can be seen here: ${ }^{44}$

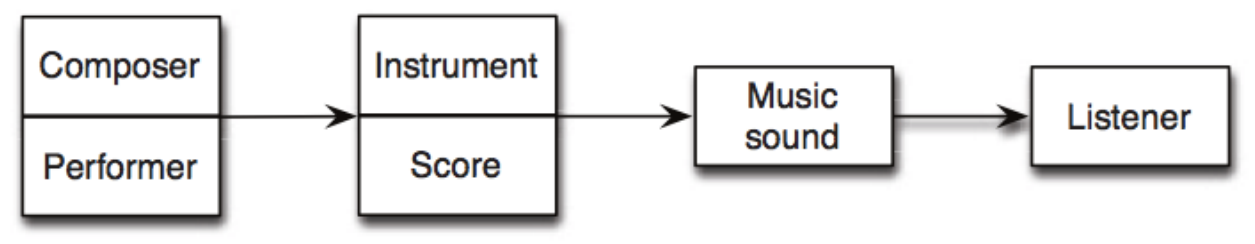

FIGURE 4: ALTERNATIVE MODEL 45

These conventional schematics of musical production (which, significantly, do not accommodate feedback) provide a picture the role musical instrumentality can play in music. They also indicate how instrumentality might have more or less focus in different

\footnotetext{
${ }^{43}$ Tellef Kvifte, "What Is a Musical Instrument?," Svensk Tidskrift För Musikforskning 90, no. 1 (2008), 50 .

${ }^{44}$ Action is not included in these models (a serious omission) but it is considered implicitly as an adjunct of the composer, score, and instrument categories. The distributed nature of action, and its ability to cut through these categories while retaining intent is part of its interest.

${ }^{45}$ Kvifte, "What Is a Musical Instrument?," 50.
} 
scenarios. Whatever the configuration and ratio, sound production, action, and ultimately music are inseparable from and dependent upon the instrument and some form of instrumentality.

The advantage for a materialist musical theory integrating the idea of actants is clear: to think musical instruments as actants can help create a more complex and nuanced concept of how distributed agency functions and set the stage for a clearer picture of instrumentality. Integral to this is to permit less concern with explicit authorial, subjective intent, and instead imagining composition as a nexus of human and nonhuman factors that collaborate in bringing musical structure into being.

Within such an expanded field of musical composition, it would seem that instruments occupy a rarely considered, interstitial space. It is typical to consider musical instruments as passive carriers of compositional, authorial, or performative intent - as a prosthesis, or extension of the musician or as passive conduits for musical thought. ${ }^{46} \mathrm{I}$ would, however, like to question this view and make a case for importance of the nonhuman in this scenario. Far from being inert carriers of intent, tools such as musical instruments are complex manifestations of the human and the non-human, inextricably enmeshed in a network of contexture. A consideration of instrumentality allows for an expansion of what is construed as compositional activity.

The focus in musical scholarship is typically on either the poietic intent of the creators or on the esthesic - the perceptual or interpretive side. Some work has been done on imminent level analysis, though such work less common. However, even these categories are not truly satisfactory. The nature of such music is hybridized and

\footnotetext{
${ }^{46}$ Matthais Haenisch, "Materiality and Agency in Improvisation: Andrea Neumann's 'Inside Piano,"” in Noise in and as Music, eds. Aaron Cassidy and Aaron Einbond (Huddersfield: University of Huddersfield Press, 2013), 149.
} 
distributed. In acoustic music especially, the instrument is simultaneously the ground or conduit of the activity (an immanent fact as it were) and a possible poietic "actant." ${ }^{47}$ On esthesic side - the side of interpretation - the instrument is an immanent fact that gives rise to normative expectations and the possibility of their subversion. If we allow an object like a musical instrument some type of agency, we can position it as the modulator of the poetic and the immanent. It is the intriguing border area between the poetic and the immanent, and the immanent and the esthesic that seems worthy of further critical investigation.

\section{In Musicology}

There has recently been some pushback on some related issues within from musical scholarship. Regarding the elements of the materialist triad (action_instrument-sound), sound and its quarrelsome cousin noise have already received thorough treatmentsscientifically, aesthetically, and at a variety of points in between.$^{48}$ Action in music also has received attention, though to a lesser degree than sound. ${ }^{49}$ Musicologists and composers grounded in ecological psychology (like Eric Clarke, Newton Armstrong, and Juraj Kojs who we will see in Chapter 4) have made important contributions to conceiving action in music. Their work is especially notable for the introduction of the concept of affordances into music.

In a broad sense, the ecological model upon which these concepts are based seems

\footnotetext{
${ }^{47}$ In contemporary music, the nature or absence of instrumentality is also significantly found in its negative, as the absence of overt instrumentality as in acousmatic music.

${ }^{48}$ See below.

${ }^{49}$ This topic will be revisited in detail Chapter 4.
} 
ideally positioned to account for the materialist elements present in the ecosystem of sound and composition. However, in practice, ecological psychology generally ignores such elements. While this work certainly opens the door for discussions of instruments and instrumentality, it focuses either on the actions of the performer on the one hand or on the experience of the listener or audience within the ecological frame, thus sidestepping the performative or compositional perspective. However such work is still a very important basis for conceiving instrumentality. Action, instrument, and sound are of course intimately related, though of course not at all equivalent in all cases. Connections to concepts of action are essential, even if this is not the primary objective here. Furthermore, action is an important common ground between Heidegger, Latour, and ecological psychology, and is moreover integral to how they conceive of objects. In conventional musicology, the presence of the body, embodiment, and physicality has been recently established. ${ }^{50}$ Aspects of this originate in the influential writings of Julia Kristeva and Roland Barthes, who famously suggested a theoretical valence for the "grain of the voice." ${ }^{\text {51 }}$ Despite drawing attention to the need to attach an aesthetic value to the unique haecceity of the performative moment and the instrumentalized voice, this concept has not produced many actionable analytical methods. However, in this search for a concept of instrumentality, establishing a parallel "grain of the instrument" is a good place to start.

Other work, like Elisabeth LeGuin's Boccherini's Body, attempts to establish a place for the oddness of bodily and gestural transference in classical performance; or, as

\footnotetext{
${ }^{50}$ For a summary of recent work on embodiment, see Fred Maus, "Somaesthetics of Music," in Action, Criticism, \& Theory for Music Education Volume 9, No. 1, January 2010, 9-25, http://act.maydaygroup.org/articles/Maus9_1.pdf.

${ }^{51}$ Roland Barthes, "The Grain of the Voice," in Image Music Text, trans. Stephen Heath (London: Fontana Press, 1977), 179-189.
} 
she puts it, a type of "carnal musicology." ${ }^{52}$ LeGuin's carnal musicology serves as a good example of this materialist blind spot. Boccherini's Body explores the embodied, temporally vexing experience of reviving a long-dead composer's gestures from the past, via notation and the body, and bringing them into living performance. However, despite chapter titles such as "Cello-and-Bow Thinking," the instrument, unfortunately, plays a minimal role here: first as a sketchpad for musical ideas preceding notation in Boccherini's case, and second as merely a hurdle to navigate as the musicking body translates gesture back into sound. Yet the instrument-the method, vehicle, and landscape of this transformation -is still mostly a blank. LeGuin's focus is on the intimate transaction involved in the musical transmutation of gesture, foregrounding an almost erotic phenomenal experience. Section titles such as "Historicizing the Terms of Embodiment" or "Carnality and the Compositional Process" bear this out..$^{53}$

A more systematic approach to understanding performativity, which has strong implications for instrumentality, can be seen in the analytical and theoretical work of contemporary theorists like Nick Cook, who makes the case for the recognition of performance as creative act. ${ }^{54}$ Other related work includes Joseph Straus' mostly musictheoretical concepts of musical normativity and disability in Extraordinary Measures, Tomi Hahn's research into enactive processes and performance, and David Sudnow's classic quasi-phenomenological account of learning jazz piano, Ways of the Hand.$^{55}$

\footnotetext{
${ }^{52}$ Elisabeth Le Guin, Boccherini's Body: An Essay in Carnal Musicology (Berkeley: University of California Press, 2006), 3. Intriguingly, this term echoes a similar one, "carnal phenomenology," found in object-oriented philosopher Graham Harman's work (See Chapter 3).

${ }^{53}$ LeGuin, Boccherini's Body.

${ }^{54}$ Nicholas Cook, Beyond the Score: Music as Performance (New York: Oxford University Press, 2013).

${ }^{55}$ David Sudnow, Ways of the Hand: The Organization of Improvised Conduct (Cambridge, MA: Harvard University Press, 1978).
} 
These are of indirect interest as the role of the instrument itself is only dealt with indirectly.

In the final analysis, within musicology, the musical instrument has yet to be properly considered in even the largely marginal ways that the music making body has, despite the emergence of these multiple new discourses around the body, embodiment, performance, and action. The significance of instruments and instrumentality in music, especially paramount in avant-garde and experimental music in which the instrument is unmistakably foregrounded, is rarely recognized. If the instrumentalized voice is accorded a grain, I posit that musical instruments - that unusual liminal zone between the human and non-human on which so much music depends - deserves one as well.

\section{Philosophical Musicology: The Composite Body}

More radical, and perhaps more promising, are musicologist Amy Cimini’s ideas regarding musical analysis and performance. Cimini, writing in reference to the confluence of thought between philosophers Giles Deleuze and Baruch Spinoza, claims we cannot think of bodies, minds, and instruments as qualitatively different and that therefore the realm of musical instruments cannot be separated from the "composite body" of music making:

"Listeners, performers, instruments, spaces, musical forms, and sonorous vibration are ontologically united, distinguished only on the basis of their capacity to effect and be effected by another. Studying a scene of listening and/or performance Spinozistically would entail unpacking the mutually affective play between and amend these capacities. Performance and/or listening then becomes nothing more and nothing less than the joining together of many always already ontologically united ideas to form a large, extremely complex composite body held together by an equally complex network of compatible, yet infinitely variable, ratios of motion to rest. The creation of the composite body allows the 
heterogeneous elements that compose it to express shared, emergent affinities that otherwise remain unrealized - affinities that, in a musically complex body would express mutual affectivities between, for example, player and instrument, listener and instrument, instrument and air, sound particle and wave." ${ }^{, 36}$

This Spinozistic-Deleuzian model, though quite metaphysical in nature, nevertheless summarizes a type of approach that can more effectively accommodate establishing a value of instrumentality. The Spinozistic "composite body" Cimini describes opens up the possibility of de-heirarchicalized relations between heterogeneous elements of an assemblage. It also presents a framework for thinking music, and by necessity musical instruments, ontologically. Thinking in terms of such a model, we take advantage of the possibilities for Spinozistic musicology and composite body that Cimini suggests. And, indeed, it can helpfully point towards a larger concept of how to reimagine composition in the expanded field.

\section{Materialist Approaches}

"There are three bodies: the body of the performer, the body of the instrument, the body of knowledge." ${ }^{57}$

\section{A Political Economy of Music}

There have been rare writers that consider musical instruments and instrumentality as essential, and moreover inseparable from the vital issues of what contemporary composition means. Economist and one-time musicologist Jacques Attali presents just such a case in his eccentric, quixotic, yet highly influential book Noise: The Political

\footnotetext{
${ }^{56}$ Amy Cimini, "Giles Deleuze and the Musical Spinoza," in Sounding the Virtual: Gilles Deleuze and the Theory and Philosophy of Music, eds. Brian Clarence Hulse and Nick Nesbitt (Farnham, England, Burlington, VT: Ashgate, 2010), 137.

${ }^{57}$ John Corbett, "Ephemera Underscored: Writing Around Free Improvisation," in Jazz Among the Discourses (Durham: Duke University Press, 1995), 226.
} 
Economy of Music..$^{58}$ In this book, he presents musical instruments not only as a potential physical and conceptual site of resistance and change but also as an arena of research. In his words, music should remind us that: "each instrument, each tool, theoretical or concrete, implies a sound field, a field of knowledge, an imaginable and explorable universe. ${ }^{\prime 59}$ Music to Attali is a prophetic reflection of the world and of our economic systems, and musical instruments are a site where new codes begin to take shape and the old codes are destroyed. Musical instruments literally make noise. Attali also sees their creation and control as a repression of noise, a channeling of it. In the cultural sphere, their decontexualization and reinstrumentalization in the cultural sphere is also a type noise.

Steve Waxman, another author for whom instruments are a concern, utilizes Attali's ideas as a jumping-off point in his book on the electric guitar, Instruments of Desire. He interprets Attali to mean that instruments give us the necessary tools to theorize about music through music itself..$^{60}$ Waxman amalgamates both Attali and John Corbett, stating that musical instruments: “... are the nexus at which the abstract codes of music-making meet the material acts through which music is produced." ${ }^{\circ 1}$ This statement contains two very important ideas. First is the idea of the instrument as a site of what can be called the composite body - the musical instruments as discrete, physical objects that have an intimate, transformative relationship with the human body and the body of

\footnotetext{
${ }^{58}$ Attali, Noise: The Political Economy of Music, Theory and History of Literature Volume 16, trans. Brian Massumi (Minneapolis: University of Minnesota Press, 1985).

${ }^{59}$ Attali, Noise, 133.

${ }^{60}$ Steve Waksman, Instruments of Desire: The Electric Guitar and the Shaping of Musical Experience (Cambridge, MA: Harvard University Press, 2000), 10-12.

${ }^{61}$ Waksman, Instruments of Desire, 11.
} 
cultural knowledge, both of music at large and of the instrument specifically. ${ }^{62}$ Any account of instrumentality must therefore account for this complex interplay of this composite body. Metaphorically, we might think of musical instruments like a complex number in mathematics: they have imaginary, non-physical components (their notation, their history, their implied body schema) and real components (their presence in physical reality; their sense data). Any accounting of instrumentality is incomplete without combing both. There must be recognition of instruments both as a site of material acts of music and as recordings of a material history of acts themselves, a type of "congealed labor" temporarily frozen in a fixed form ${ }^{63}$ For performance through an instrument is of course a material act, but the building itself is also an act of design and construction.

Both of these elements point to what is interesting about them musical instruments as tools within music. However Attali's understanding of instruments is couched in highly relational terms of postmodern discourse. Though he strongly advocates a materialist dimension for music - he was an economist after all - he never does quite arrive. Written in 1985 within the French poststructuralist milieu, there is a definite focus on discourse and language. This should come as no surprise. Materialist concerns in academic discourse were at a nadir around the time of this book's composition, in favor of poststructuralist and deconstructionist perspectives based on language, discourse, and subjectivity. Perhaps though this is one reason why Noise has remained in academic circulation despite its flaws. In clearing out a theoretical space for instruments, Attali suggests the possibility of a neo-materialist reappraisal of musical

\footnotetext{
${ }^{62}$ Corbett, "Ephemera Underscored," 226.

${ }^{63}$ Bruno Latour, Pandora's Hope: Essays on the Reality of Science Studies (Cambridge, MA and London: Harvard University Press, 1999), 189.
} 
instruments, while furthermore identifying the role they could play in less market-driven forms of music composition.

A key equivalence in Attali that points to here is between "instrument" and "tool". If we allow that instruments are a type of tool, then what kind of tool are they? And why is it that they are so often ignored within musical scholarship? Music is of course not alone in this underestimation of the significance of its own tools, far from it. There is starting to be a widespread recognition across a number of fields that our understanding of tool usage and technology - and within these I include instruments generally and musical instruments specifically —is wanting. There are however interesting recent trends in that can perhaps shed light on musical instruments, instrumentality, and their role within the interrelated domains of action, instrument, and sound.

\section{Musicking}

Paradoxically, it is the attempt to open up music into a more complex, multi-laminar, social endeavor-encapsulated Christopher Small's term musicking - that opens up a theoretical basis that better accounts for a materialist reading. For instance, Small postulates:

To music is to take part, in any capacity, in a musical performance, whether by performing, by listening, by rehearsing or practicing, by providing material for performance (what is called composing), or by dancing. ${ }^{64}$

From this perspective it seems a small but essential step to consider the non-human elements, such as objects, instruments, architecture, and even physical forces, as

\footnotetext{
${ }^{64}$ Christopher Small, Musicking: The Meanings of Performing and Listening (Hanover, NH: University Press of New England, 1998), 8.
} 
significant in this scenario. Small claims that anything that organizes and acts in a performance matters, expanding the definition of music to encompass all contributing acts as relational, which in turn metaphorically model ideal relationships "between person and person, between individual and society, between humanity and the natural world and even perhaps the supernatural world. ${ }^{\circ 5}$ In his focus on acts, and its implications of differentiation between human and non-human elements, Small misses the ways in which, in much music, the relationship between individual and society or humanity and the natural world is not serving as a metaphor, but in some cases actually form the structure and the substance of the music. If we allow ourselves to consider musical instruments as dynamic, active, social objects, a materialist musical theory seems not only a reasonable but also a necessary intervention.

\section{Ethnomusicology and Material Musical Culture}

Within musical scholarship, it is ethnomusicology points most promisingly towards other possibilities. Despite the fact that organology has largely abdicated any ontological or compositional considerations in favor of taxonomies and histories,${ }^{66}$ Curt Sachs' monumental History of Musical Instruments briefly but admirably attempts to summarize the entire human history of musical instrument usage. Sachs places the instrumental impulse as originating inherently as an extension of the body, citing the unavoidable expressivity of movement and gesture ${ }^{67} \mathrm{He}$ however has little interest in any other materialist effects. More usefully, Alan P. Merriam, in his Anthropology of Music, insists

\footnotetext{
${ }^{65}$ Small, Musicking, 13.

${ }^{66}$ Margaret J Kartomi, On Concepts and Classifications of Musical Instruments, Chicago Studies in Ethnomusicology (Chicago: University of Chicago Press, 1990).

${ }^{67}$ Curt Sachs, The History of Musical Instruments (New York: W. W. Norton \& Company, Inc, 1940), 26.
} 
that it is incumbent upon ethnomusicologists to consider "musical material culture" in their analysis. ${ }^{68}$ This includes not only taxonomies, but also topics like special treatment of instruments, symbolic meanings, and sculptural implications, as well as means of instrumental production and other economic aspects. ${ }^{69}$

Paul Théberge, in his superb analysis of music and technology Any Sound You Can Imagine builds on Merriam's idea, utilizing components of Merriam's analytical model. ${ }^{70}$ While stating a number of reservations about Merriam's overall analytic, Théberge nevertheless makes use of this concept of material musical culture in his own work regarding music technology in the 1970s and 1980s, while suggesting that this consideration of material musical culture is fundamental to the understanding any music, especially when it allows a "working backwards" from materialist elements to the level of musical concepts. ${ }^{71}$ Théberge notes that musical instruments: "take part in a dynamic interplay with musical concepts at the most fundamental level. Material culture and abstract systems of musical thought and organization thus form a dialectal relationship of the utmost important in music-making." ${ }^{2}$

More closely aligned with establishing what he calls a new organology is the work of Allen Roda. He suggests an essentially organological intervention but of a highly theoretical variety, stating:

"I propose that by studying the intimacy of [...] sonic relationships, the physical experience of bodies interacting, and the cultural and intellectual knowledge that

\footnotetext{
${ }^{68}$ Alan P Merriam, The Anthropology of Music (Evanston, IL: Northwestern University Press, 1964$), 45$.

${ }^{69}$ Merriam, Anthropology of Music, 45.

${ }^{70}$ Paul Théberge, Any Sound You Can Imagine: Making Music/Consuming Technology, Music/culture (Hanover, NH: Wesleyan University Press, published by University Press of New England, 1997), 157166.

${ }^{71}$ Théberge, Any Sound You Can Imagine, 162-163. Théberge claims it is too functionalist, to linear-and indeed notes that Merriam himself underuses these resources in his own analyses.

72 Théberge, Any Sound You Can Imagine, 163. This model is remarkably similar to Manuel DeLanda's concept of the assemblage, drawn from the philosophy of Giles Deleuze and Felix Guitarri.
} 
musical instrument embody and transfer, the musical instrument - human relationship could be a unique realm of analysis for a new organology that both draws from and contributes to an interdisciplinary approach to the human/non-human relationship. In order to understand the relationship between humans and musical instruments it will be necessary for organologists to use tools and methodologies from other disciplines such as the anthropology of material culture, actor network theory, and phenomenology."73

Roda stops short of positing a concept of instrumentality or advocating an ontological intervention per se (and certainly is not interested in issues related to composition) but his suggestion that we adopt tools and methodologies from actor-network theory and phenomenology clearly point towards both the a desire for something more comprehensive.

Ethnomusicologist Andy Nercessian articulates a similar concern. In his book The Duduk and National Identity in Armenia, Nercessian briefly tackles similar problems of organology. Nercessian, frustrated with the typical focus in ethnomusicological literature on physical characteristics as opposed to acoustic ones, proposes an instrumental "trialectic" which views a musical instrument as best understood as a combination of (1) its materialist considerations; (2) the context in which is has meaning; and (3) the subject, audience, or beholder of the instrument. ${ }^{74}$ Nercessian's concept of materiality and the ways instruments effect musical discourse is limited however. His desire to focus on acoustic characteristics immanent in the instrument's construction is important, but he seems ultimately uninterested in teasing out the implications of such elements. ${ }^{75}$

Perhaps the most profound and poetic attempt in this regard has been Paul

\footnotetext{
${ }^{73}$ Allen Roda, "Toward a New Organology”, November 21, 2007, http://allenroda.com/toward_alla_new_organology/)."

${ }^{74}$ Andy Nercessian, The Duduk and National Identity in Armenia (Lanham, MD: Scarecrow Press, 2001), 7-16.

${ }^{75}$ Nercessian, Duduk and National Identity. The subsequent book after the introduction says very little about the materiality or instrumentality of the Duduk.
} 
Berliner's Soul of Mbira. Relentlessly circling around the multifarious manifestations of both instrument itself and it practitioners, Berliner examines every part of the culture, construction, and music related to the Mbira. The instrument is presented as an object that resides in a complex domain, at once a device and a conduit; a test, a challenge; a technology and an old friend; a field of activity, a place; totem and a symbol, both in sound and object. A musical instrument a tool that is both created and creates an inner space.$^{76}$ There is no type of philosophical or ontological discussion in Soul of Mbira. Instead we have a comprehensive, participatory account hat points to the indissolubility of the material and non-material elements, a confirmation of Latour's assertion about anthropologists being the main pre-practioners of bridging the subject-object and natureculture divides..$^{77}$

\section{The Non-Human Turn}

"To balance our accounts of society, we simply have to turn our exclusive attention away from humans and look also at nonhumans. Here they are, the hidden and despised social masses who make up our morality. They knock at the door of sociology, requesting a place in the accounts of society as stubbornly as the human masses did in the nineteenth century. What our ancestors, the founders of sociology, did a century ago to house the human masses in the fabric of social theory, we should do now to find a place in a new social theory for the nonhuman masses that beg us for understanding." ${ }^{178}$

That such a fractured discourse in musical scholarship has yet to coalesce into a focused consideration of the significance of the materials of musical production, and far less one

\footnotetext{
${ }^{76}$ Paul Berliner, The Soul of Mbira: Music and Traditions of the Shona People of Zimbabwe, Perspectives on Southern Africa; 26 (Berkeley: University of California Press, 1978).

${ }^{77}$ Bruno Latour, We Have Never Been Modern, 7. "In works produced by anthropologists abroad, you will not find a single trait that is not simultaneously real, social and narrated." However he also later states in this book that anthropologists typically stumble over technology and science, and therefore the anthropological model is unable to account for the modern, which is inherently filled with technology and "quasi-objects" See Latour. We Have Never Been Modern, 91.

${ }^{78}$ Bruno Latour, "Where Are the Missing Masses? The Sociology of a Few Mundane Artifacts," in Shaping Technology, Building Society: Studies in Sociotechnical Change, ed. Wiebe E. Bijker (Cambridge, MA and London: The MIT Press, 1992), 227.
} 
specifically geared towards musical instruments and instrumentality, is no surprise.

However, recent developments in philosophy, psychology and critical theory have shed new light on the significance of "things"-objects, instruments, and technology ${ }^{79}$. Scholar Richard Grusin traces a recent "non-human turn" in a wide variety of twentieth century studies, including actor-network theory ("Bruno Latour's career long project to articulate technical mediation non-human agency, and the politics of things") but also affect theory, animal studies, assemblage theory, recent brain sciences, new media theory, speculative realism, object-oriented philosophy, and systems theory. ${ }^{80}$ Not included in Guisin's list, but work is also significant if not well known, are theorists of technology, such as Bernard Stiegler (whose philosophy of technology is a continuation of Jacques Derrida), Jacques Simondon, theorist of technology Martin Feenberg; and political scientist Jane Bennett turned analyst of things and "vibrant matter."

\section{New Materialism}

This constellation of thinkers bound together by materialist concerns are grouped together as movement called New Materialism. Definitions of just what this encompasses differ, but very generally is a trend in the humanities that is a response to the cultural turn in the 1970s that privileged language, discourse, culture and values. The history of the term goes back to the 1990s:

"Manuel DeLanda and Rosi Braidotti-independently of one another-first started using "neo-materialism" or "new materialism" in the second half of the 1990s, for a cultural theory that does not privilege the side of culture, but focuses on what Donna Haraway (2003) would call "naturecultures" or what Bruno Latour simply referred to as "collectives" ([1991] 1993). The term proposes a cultural theory that radically rethinks the dualisms so central to our (post-)modern thinking and always starts its

\footnotetext{
${ }^{79}$ See Jane Bennett, Vibrant Matter: A Political Ecology of Things (Durham: Duke University Press, 2010).

${ }^{80}$ Richard Grusin, "Introduction," in The Nonhuman Turn, ed. Richard Grusin, Center for 21st Century Studies; 21st Century Studies (Minneapolis, London: University of Minnesota Press, 2015), vii - xxix.
} 
analysis from how these oppositions (between nature and culture, matter andmind, the human and the inhuman) are produced in action itself."

It is a cross-disciplinary push back against the forces of post-structuralism through reclaiming creative power of matter, objects, and things were ignored in favor of a postmodernist, deconstructionist discourse.

The crux of these New Materialisms can be thought of as a denial of an Enlightenment idea that underpins historical modernism. A situation arises in the thought of Emmanuel Kant who, as the neo-Heideggerian, anti-correlationist argument goes, brackets all existence as a subset of human knowledge..$^{82}$ Therefore ontology (what is in the world) and epistemology (what we know) are separate and do not effect one another. ${ }^{83}$ In other words, if all of our understanding of the world is posited on our own perception, then ontology is irrelevant and things-in-themselves unknowable. Everything then is a type of epistemology. As the argument goes, things-in-themselves are unreachable and can never be known, and, in a very real way, are not important.

A common thread amongst such New Materialisms then is that correlationism is largely responsible for the almost complete erasure of ontological thought until recently. ${ }^{84}$ While I am suspicious about that levels to which some thinkers expand this argument, it nevertheless true that this Kantian influence is central to modernism writ large. As a result, it is difficult to fully think about objects, to think things, and to allow them a more dynamic and complex, even creative existence. It also seems clear that such a lines of

\footnotetext{
${ }^{81}$ Rick Dolphijn, Iris van der Tuin, and OAPEN, New Materialism: Interviews and Cartographies (Open Humanitites Press [Imprint], 2012), 93.

${ }^{82}$ See Graham Harman, "Brief SR/OOO Tutorial," Object-Oriented Philosophy, n.d., https:/doctorzamalek2.wordpress.com/2010/07/23/brief-srooo-tutorial/. See also Graham Harman, Prince of Networks: Bruno Latour and Metaphysics, Anamnesis (Prahran, Vic: re.press, 2009), 123-127.

83 "Newmaterialistscartographies - New Materialism," accessed April 21, 2017, https://newmaterialistscartographies.wikispaces.com/New+Materialism.

${ }^{84}$ Rick Dolphijn, Iris van der Tuin, and OAPEN, "Interview with Quentin Meillassoux," in New Materialism: Interviews and Cartographies (Open Humanities Press [Imprint], 2012), 71-75.
} 
thought, dominant in modernism, seem to have placed artificial borders between the human and non-human realms in pointless and misleading ways. In the words of Manuel DeLanda, neo-materialist intervention attempts to implicitly and explicitly ward off modernist oppositions and show us how "organic and inorganic matter, in their entanglement, create the new." ${ }^{\circ 5}$

Elements of these neo-materialist trends present an intriguing basis for the development of a concept of musical instrumentality. Specifically, in this dissertation I borrow from the bodies of knowledge and thinkers that are foundational to the current neo-materialist thinking, that point most directly towards a neo-materialist reappraisal of the creative properties of the instrument itself. Of special interest then are theories that involve instruments, tools, equipment, and objects.

In particular, Heidegger and Latour share related approaches focus on a type of "techno-phenomenology" that seeks to overcome the subject-object divide that characterizes modernism. ${ }^{\mathbf{8 6}}$ For the purposes of musical instrumentality, these can be divided up into three interrelated domains: (1) The concepts of actants, quasi-objects, and hybrid actors from actor-network theory, primarily the work of Bruno Latour, which foregrounds epistemological problems of objects within a cultural critique of modernism

\footnotetext{
${ }^{85}$ Rick Dolphijn, Iris van der Tuin, and OAPEN, "Interview with Manuel DeLanda," in New Materialism: Interviews and Cartographies (Open Humanitites Press [Imprint], 2012), 43.

${ }^{86}$ There are a number of recent articles that draw attention to the similarities, differences, and overlaps between the ontology of Heidegger and Latour - from social science: Søren Riis, "The Symmetry Between Bruno Latour and Marin Heidegger: The Technique of Turning a Police Officer into a Speed Bump," Social Studies of Science 38, No. 2 (April 2008), 287-301; Jeff Kochan, "Latour's Heidegger," in Social Studies of Science, Vol. 40, No. 4 (August 2010), 579-598; and Arianne Conty, "Techno-Phenomenology: Martin Heidegger and Bruno Latour on How Phenomena Come to Presence," South African Journal Of Philosophy 32, no. 2 (2013): 211-326; from architecture and design an example is Neil Leach, "Digital Tool Thinking: Object Oriented Ontology versus New Materialism" (Acadia 2016: Posthuman Frontiers: Data, Designers and Cognitive Machines, University of Michigan Taubman College, Ann Arbor, Michigan (October 27-29), 344-51, http://papers.cumincad .org/data/works/att/acadia16_344.pdf. This general overlap also occupies a good portion of Graham Harman, Prince of Networks: Bruno Latour and Metaphysics, Anamnesis (Prahran, Vic: re.press, 2009).
} 
that seeks to establish a new, hybrid "natureculture" relationships which problematize the subject-object relationship; (2) Martin Heidegger's ontological theories of tools/equipment and his neo-Heideggerian disciples and detractors; and (3) Concepts drawn largely from ecological psychology. All of these are broadly augmented by ideas from phenomenology and musical scholarship, especially Adorno. For the sake of convenience, I will use the term New Materialism/neo-materialism rather broadly to refer to this combination of actor-network theory, neo-Heidegerrianism, and other related trends listed above. Together, I will to show how these ideas provide a more practical methodology for reframing and recontextualizing musical instruments in the compositional process, resulting in a functional instrumentality. Interpreting music as an assemblage of mediators, or assemblage of actants, can provide a ground for understanding instrumentality and its relationship to composition to our increasingly technologized, and disciplinarily Balkanized, contemporary musical environment.

It is my hope that this neo-materialist concept of instrumentality can both provide a different reading of the value of the experimental and avant-garde, but also help us embrace more diverse musical practices and their place within contemporary composition. I hope to show how such falsely rarified work is in fact more in tune with the outside world than often imagined. It is not my intent to diminish other strategies or perspectives. To paraphrase of Rita Felski, through undertaking a materialist analysis rather than pitting it against other hermeneutical strategies, I hope to build better bridges between them ${ }^{87}$ Like Felski, I do not seek to shut out the larger world but, in fact, to let it in.

\footnotetext{
${ }^{87}$ Rita Felski, Uses of Literature, Blackwell Manifestos (Malden, MA, Oxford: Blackwell Pub, 2008), 13.
} 


\section{Here Comes Everything Modernist Aesthetics and the Total Configuration}

\section{A Fetishism of Means}

"Do you think I care about your wretched fiddle when the spirit takes hold of me?"

- Comment attributed to Ludwig Van Beethoven

"So much the worse for wood when it finds itself a violin." - Arthur Rimbaud, in a letter to Paul Verlaine

One of Theodor Adorno's principal criticisms of Igor Stravinsky in The Philosophy of New Music is that in Stravinsky's music, the instruments take precedence over the composition thereby producing music of a lesser, purely material character ${ }^{88}$ This criticism, one in a fusillade of criticisms launched in Stravinsky's general direction, is directed specifically towards the compositions The Soldier's Tale and The Rite of Spring, but runs through Adorno's writings not only on Stravinsky but on modern music and art in general. This attitude represents a formalization of the typical point of view regarding musical instruments: that they are passive intermediaries of expression. Adorno's overall

\footnotetext{
${ }^{88}$ Adorno, Theodor W. Philosophy of New Music, trans. Robert Hullot-Kentor (Minneapolis: University of Minnesota Press, 2006), 128-129.
} 
position here and elsewhere is quite clear: such a focus on instruments, their sounds, and their materiality results in a "fetishizing." ${ }^{\circ 9}$ Musical instruments and their sounds can only be a medium, as opposed to a valid material. He places them in direct opposition to compositional structure and musical meaning, in a dialectical opposition of the fetishized sound and the demands of compositional structure..$^{90}$ A priori, Adorno rejects the inherent value of musical instruments and indeed in sound or noise.

In a later work, Aesthetic Theory, Adorno goes even further, writing off all art that uses "crudely physicalistic procedures in the material" to "become what art cannot becanvas and pure tones." ${ }^{11}$ This statement-itself a crude caricature of the art practices came about in the late 1950s and 1960s - misunderstands and misrepresents the profoundly new aesthetics that began in the postwar years, aesthetics on which some the most enduring music and art of the twentieth and twenty-first century is based. Indeed, the accession of the materials of production and very processes of art into the realm of subject matter is a central concern of modernist art and a lasting part of its legacy. ${ }^{92}$ Adorno blames this critical malfunction on Stravinsky's "animosity against the anima," which does not relate to the human being, but seeks to investigate something existent in itself as he says, resulting in a disrelation of subject and object. ${ }^{93}$

\footnotetext{
89 "The means - the instrument - are hypostatized: This takes precedence over the music," Adorno, Philosophy of New Music, 128. This attitude is also seen clearly in Adorno, Theodor W, Gretel Adorno, and Rolf Tiedeman. Aesthetic Theory. Theory and History of Literature. Minneapolis: University of Minnesota Press, 1997.

90 Théberge, Any Sound You Can Imagine, 190.

${ }^{91}$ Adorno, Aesthetic Theory, 103.

92 J. M Bernstein, Against Voluptuous Bodies: Late Modernism and the Meaning of Painting (Stanford, CA: Stanford University Press, 2006), 2.

${ }^{93}$ See Adorno, Philosophy of New Music, 129. Ironically, Stravinsky himself as a composer seemed to have little to no interest in, or outright hostility towards, either performers or instruments as contributors to the creative process. Quite the opposite: similarly to Schoenberg, viewed them antagonistically, as a necessary evil. See Bruce Ellis Benson, The Improvisation of Musical Dialogue, (Cambridge and New York:

Cambridge University Press, 2003), 12-13.
} 
Despite Adorno's disapproval, the past century of music is full of examples of musical practices that overtly or covertly prioritize the creative use of musical instruments, instrumentalized objects, or instrumentality itself as their primary subject matter ${ }^{94}$ It is worth considering that the value of such music lies precisely in what Adorno articulates as a negative: the disrelation of subject and object. Far from a fetishizing, it is precisely such an approach to the materials of art-in this case instruments themselves - that has engendered a multitude of new practices and technologies which artists and musicians have since explored.

\section{The Total Configuration and the Long Sixties}

J. M. Bernstein, in attempting to define modernist painting, says that:

"In a remarkable series of transformations, the representational content of paintings came to be marked, shadowed, or resisted through being displayed or through embodied features unique to the practice of painting: the brush stroke, the properties of the pigment, the flat surface, the shape of the support, the specific properties of color and line..$^{95}$

This "foregrounding and incorporating the components of painterly practice" is analogous to modernist musical practices, particularly in the late 1950s. Similarly to visual art, formerly obscured elements of musical practice also come more and more to the forefront as material in these postwar years. Any component of art making can be utilized as material. Any element of composition, notation and performance-and by extension sounds and the actions and instruments that make them - can be focused on and utilized. This is the logical consequence of the modernist enterprise and its drive

\footnotetext{
${ }^{95}$ Bernstein, Against Voluptuous Bodies, 2
} 
towards abstraction and formalism. This is the historical phenomenon Michael Nyman describes in his book Experimental Music when he explains: "experimental music exploits a [musical] instrument not simply as a means of making sounds in the accepted fashion but as a total configuration - the difference between 'playing the piano' and 'the piano as a sound source." ${ }^{\prime 96}$ Though he was speaking specifically in regards to AngloAmerican musical experimentalism of the 1960s and 1970s, such an approach is equally applicable to elements of the European compositional avant-garde and to U.S. Jazz, if to a lesser degree.

The emergence of instrument as total configuration in what I will term "the long sixties" (1957-1969) ${ }^{97}$ embodies the contradictions inherent in modernism that were deeply embedded in the modernist project. The total configuration is evidence of the quixotic project of high modernism, a curious combination of an all-out assault on normativity, often cloaked in the garb of objectivity and formalism. The turn into the sixties was the apex of high modernist urge towards a formalist, utopian, almost context free, “objective” art. As Graham Harman summarizes Latour's attitude towards modernism: "modernity is the impossible attempt to create a radical split between objective natural fact and arbitrary human perspective." 98

The experimental music of the long sixties (1957-1969) is representative of this crisis. The rift between all of the elements of the materialist triad and musical expression was, at the turn into the sixties, collapsing. This is the beginning of what we see for instance in Abstract Expressionist paintings, such as Jackson Pollock or Mark Rothko.

\footnotetext{
${ }^{96}$ Michael Nyman, Experimental Music: Cage and Beyond, 2nd edition; Music in the Twentieth Century (Cambridge, U.K, New York: Cambridge University Press, 1999), 20.

${ }^{97}$ This term borrowed from from Bruce J. Schulman, The Seventies: The Great Shift in American Culture, Society, and Politics, 2001 and Nadine Hubbs, Country Music for the Resistance.

${ }^{98}$ Harman, Prince of Networks, 31.
} 
There is no more separation between canvas/painting/paint on the one hand and emotion/structure/subject matter on the other. The subject-object divide is no longer sustainable. The need for a strict separation of subject and object, so important to Adorno, is identified by Bruno Latour as the problematic underpinning of the modernist project writ large..$^{99}$

\section{An Age of Wire and String}

This period of modernist art is the crucible from which the instrument-as-object ultimately emerges. An example of this can be seen in the early work of Japanese composer Toshi Ichiyanagi. In 1961 he was living in New York City and associating with John Cage and Fluxus artists. In his series of compositions Music for Piano No.l - No. 7 , all manner of playing techniques and indeterminate notations are developed ${ }^{100}$ However, No. 7 stands out in particular. Certainly there is indeterminacy involved here. But there is also something more. This piece, through its notation, requires the performer to reevaluate not only one's relationship to musical notation (and possibly performance and music for some) but indeed to the instrument itself. Notice for instance that the notation is read down the page vertically (as Japanese is traditionally read) instead of left to right as is typical of European languages. This is more reflective of one's actual physical orientation towards the keyboard while playing.

\footnotetext{
${ }^{99}$ Bruno Latour, We Have Never Been Modern, trans. Catherine Porter (Cambridge, Mass: Harvard University Press, 1993), 1-13. This argument essentially encompasses the entire book.

100 Nyman, Experimental Music, 111-112.
} 


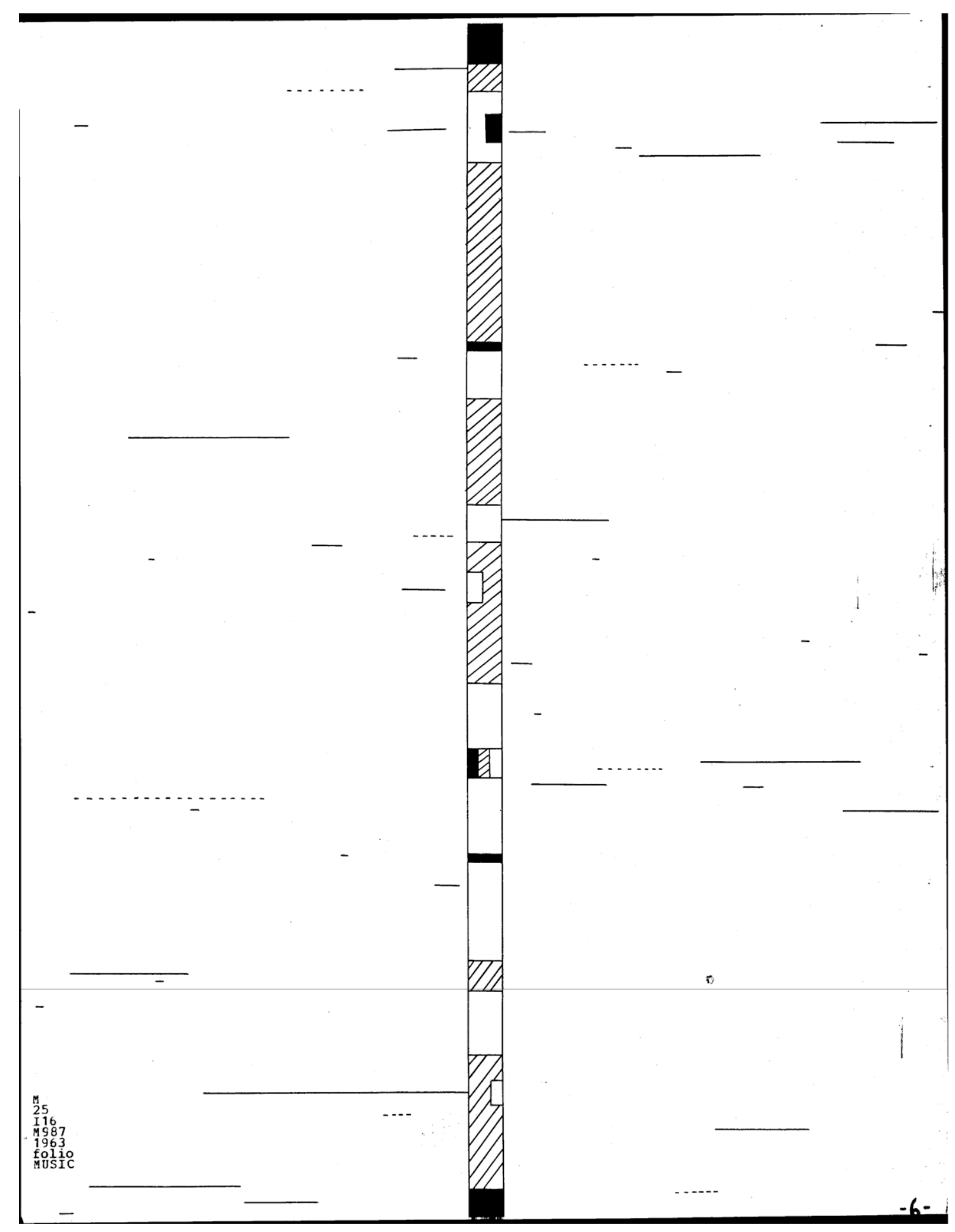

FigURE 5: EXCERPT FROM TOSHI ICHIYANAGI's MUSIC FOR PIANO NO. 7 (1961) 
This is also a similarity it shares with Labanotation, a form of dance notation developed a few decades earlier, where the physical layout mirrors the human body, as seen in the following example.
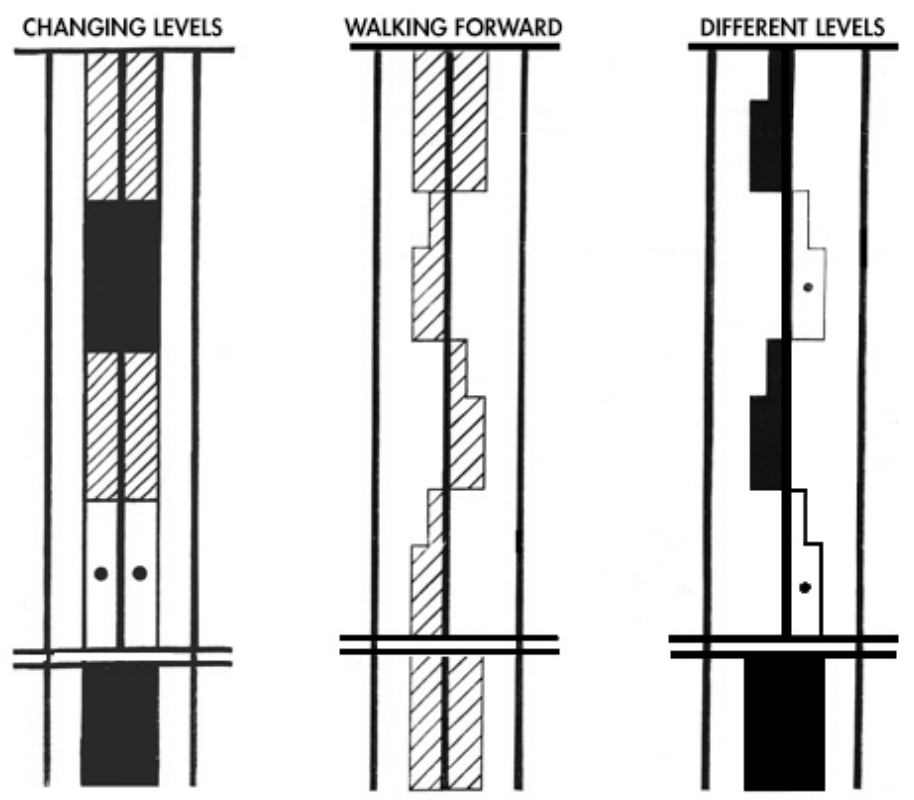

FIGURE 6: LABANOTATION EXAMPLE. ${ }^{101}$

These similarities between these two notations are as compelling as they are impossible to ignore. As Ichiyanagi was familiar with Merce Cunningham (evidenced by his piece IBM for Merce Cunningham of 1960), it is unlikely this similarity was incidental. In both cases, such notational re-mappings point towards the emergence of alternative cartographies of the performative and the instrumental body. The final result is no less than an interrogation of this composite body schema - the body of the performer, the landscape of the instrumental body, and the body of knowledge of piano playing and composition.

${ }^{101}$ Image downloaded from http://www.is-movementarts.com/labanotation/. 


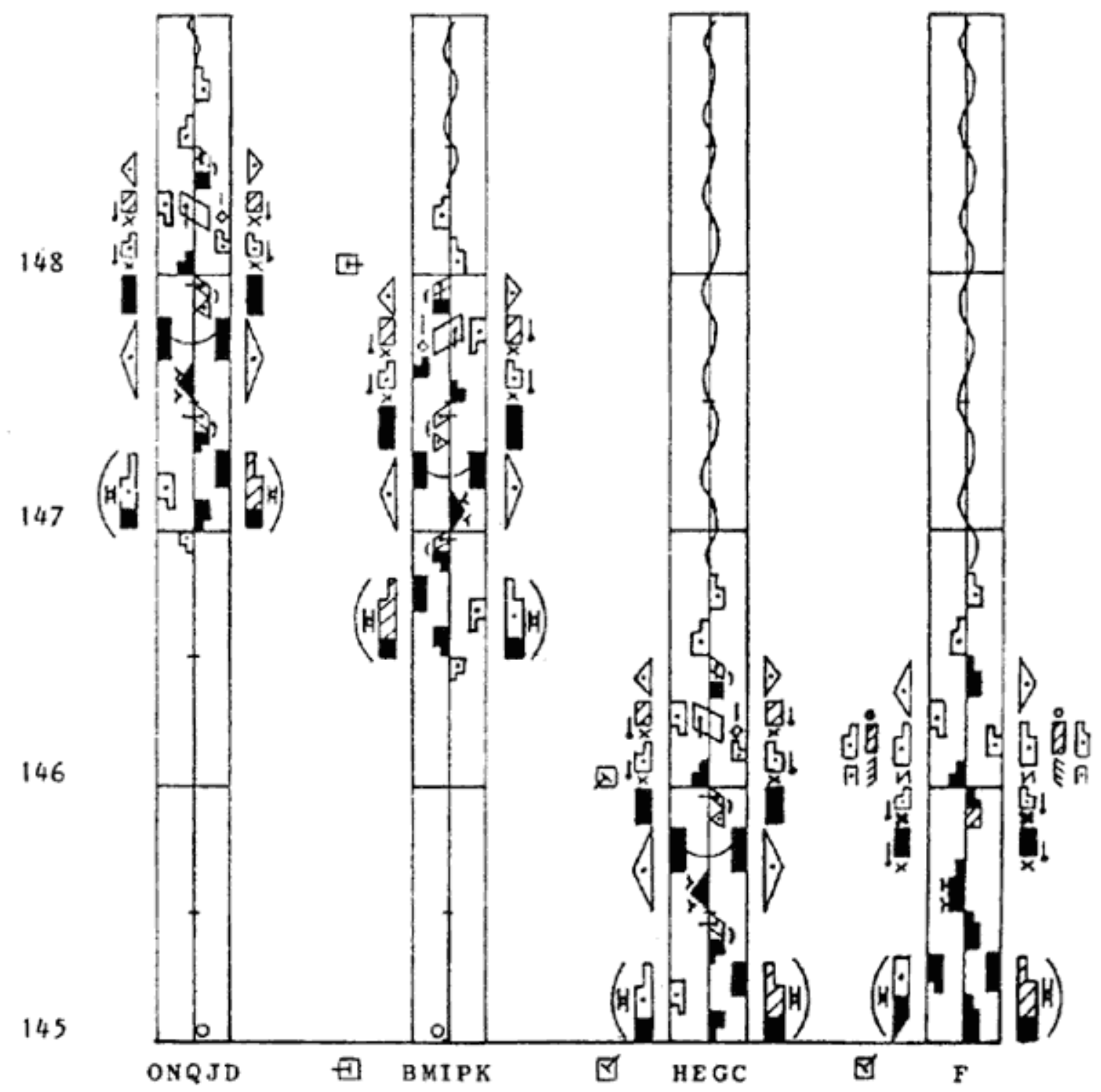

FIGURE 7: ANOTHER EXAMPLE OF LABANOTATION ${ }^{102}$

Unlike his mentor and colleague John Cage, Ichiyanagi had a very direct

connection to Japanese aesthetics. ${ }^{103}$ The Japanese concept of $m a$, or between-ness, was important to Ichiyanagi. $M a$ has been described as central to Japanese artistic subjectivity, which centers on the space between or the gap between the individual and

\footnotetext{
${ }^{102}$ Image downloaded from http://www.sportsbabel.net/2008/11/labanotation.htm

${ }^{103}$ Yayoi Uno Everett, "Toshi Ichiyanagi and the Art of Indeterminacy I Post," accessed April 22, 2017, http://post.at.moma.org/content_items/90-toshi-ichiyanagi-and-the-art-of-indeterminacy.
} 
people on the one hand and the objects the person interacts with on the other ${ }^{104}$ Related to this, Japanese aesthetics scholar Onishi Yoshinori identifies the essence of Japanese art pantomimic, since the artist strives to represent an awareness of the lack of distinction between nature, art, and life; as such, art represents an approach to the world which corresponds to actual existence, which "somehow does not discriminate between subject and object." ${ }^{105}$

Just down the street in New York City, but a world away culturally, Cecil Taylor was pursuing his innovative, improvisatory hybrid of jazz and classical music. Through an incongruous synthesis of his idols such as Duke Ellington and Béla Bartók, Taylor pursued increasingly alarming assaults on the piano keyboard throughout the $1960 \mathrm{~s} .{ }^{106}$ Operating in a space opened up by pianists Bud Powell, Thelonious Monk, Lenny Tristano, in addition to other instrumentalists from Bebop and free jazz, his uncompromising, iconoclastic approach made him both symbol of progress and pariah. However, as Taylor's body of recorded work increased and he gravitated further away from tonal structures we gradually hear more and more of the sound of the piano's totality. From playing inside of the instrument to micro-choreographies of his hands and arms on the keys, spliced together with moments of fragmentary melody and brittle harmonic markers, we begin to hear at certain moments something like the sound of the human body filtered through the keyboard's configuration. As with the cluster-based compositions of the early twentieth century, this music begins to reveal the actual structure of keyboard design and construction through a translation of micro-

\footnotetext{
${ }^{104}$ Quoted in Luciana Galliano, “Toshi Ichiyanagi, Japanese Composer and 'Fluxus'," Perspectives of New Music, Volume 44, No. 2 (Summer, 2006), 257.

${ }^{105}$ Galliano, "Toshi Ichiyanagi," 257.

${ }^{106}$ https://www .theguardian.com/music/musicblog/2010/apr/14/cecil-taylor-jazz-piano
} 
choreographies of the hand into action and gesture into sound.

Across the Atlantic in 1961, Karlheinz Stockhausen was completing his Klavierstücke $X$. Premiered by Frederic Rzewski in 1962 it consists in an all-out assault on the keyboard punctuated with large periods of resonance and silence. Sliding hand clusters, arm clusters, and extreme dynamic can be seen, all in close succession. The impression in performance is assault, violence, and violation. Despite a very different aesthetic point of origin compared to Taylor or Ichiyanagi, there are remarkable points of overlap, a similarity both in execution and in aggregate result. Physicality and the instrumental interface are front and center in this music. From this, the instrument as object emerges. The structure of this instrumental object comes to structure the sonic discourse on both the local and global levels.

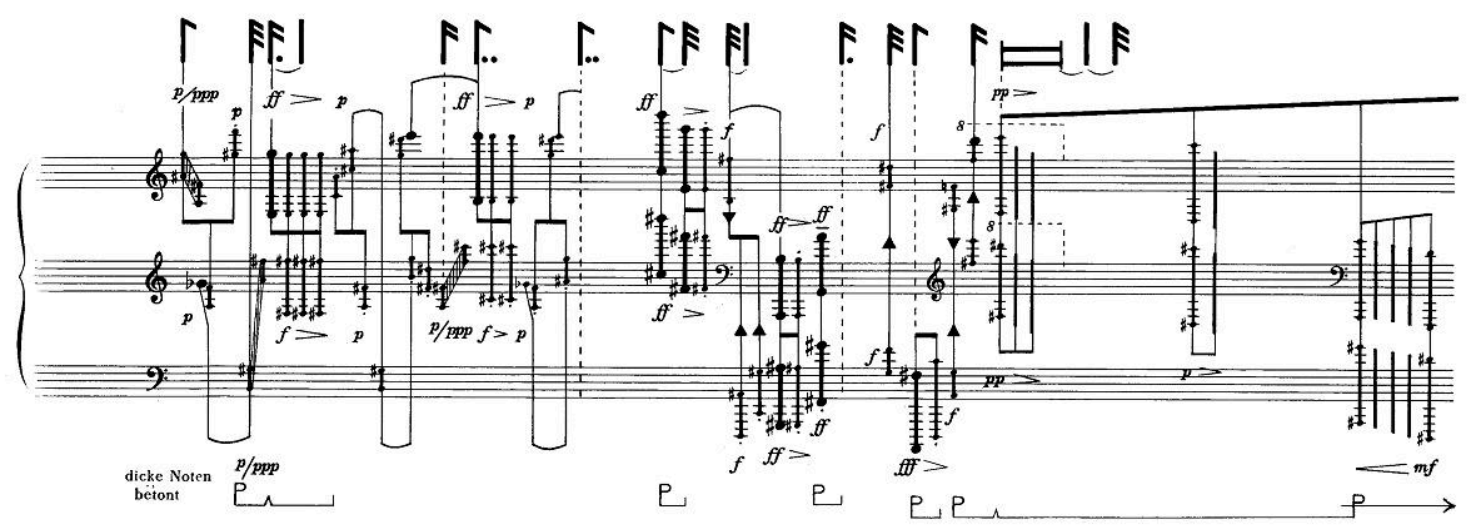

FIGURE 8.: EXCERPT FROM STOCKHAUSEN'S KLAVIERSTÜCKE X (1961).107

\section{Pianos Afford X}

The piano is an ideal instrument for such interrogations. It is one of the most object-like of musical instruments. They are large and unwieldy, with industrially produced mechanisms and often elaborate cabinetry. Unlike most other instruments, the piano

\footnotetext{
${ }^{107}$ Karlheinz Stockhausen, Klavierstücke X, Nr. 4, Universal Edition, 1967. Accessed April 22, 2017.
} 
mostly lives in the space where it is played. Indeed many pianos probably spend the majority of their lives un-played, functioning largely as a "complex piece of audio furniture." ${ }^{108}$ Its essential mechanism is percussive, a sharp and violent attack. As a result, it has always been uncomfortably situated organologically, classified somewhere between percussion, string, and keyboards. It is nevertheless the exemplar of bourgeois music making; it represents the triumph of machine and industry into the homes of the masses. ${ }^{109}$ Then and before, the piano was a kind of radio before radio. The initiated could interface with this machine, facilitating the performance and new symphonies and popular music alike. It was and still is for many the supreme compositional tool..$^{110}$ But the piano is also problematic. Its striated, grid-like tone world dominates our very conceptions of what we imagine music to be: tonal, tempered, and of discrete pitch. The reality of sound kept at an arm's length, partitioned. Noise is sequestered and hidden away. It domesticates the world of pitch, indeed of music itself.

Even before this turn into the sixties, there was, especially in American music, a long tradition of piano abuse and misuse. This begins with Charles Ives' preposterously massive chords in works like the Concord Piano Sonata (composed 1911-1915, though published in 1919), which suggests using piece of wood for a cluster chord. Stride pianists like James P. Johnson and Willie "The Lion” Smith brought a new percussiveness and physicality to the piano. The mostly forgotten composer (and incidentally, most popular American concert pianist of his day), Leo Ornstein, in pieces like Wild Men's Dance (1913-14) and Suicide in an Airplane (1913) ups the ante

\footnotetext{
108 Annea Lockwood, "How To Prepare a Piano," in Sound Scripts: Proceedings of the Inaugural Totally Huge New Music Festival Conference 2005, vol. 1, 2006, 2.

${ }^{109}$ Craig H Roell, The Piano in America, 1890-1940 (Chapel Hill: University of North Carolina Press, 1989), 3-17.

${ }^{110}$ Even in the virtual world of the laptop, the configuration of the piano keyboard still refuses to die.
} 
considerably, unleashing flurries of cluster-based violence. Later, beginning 1916, Henry Cowell experiments with tone clusters, and in 1925 composed now well-known pieces like Tides of Mananahan, with its extensive use of arm clusters, and The Banshee, famous for playing the strings inside the piano.

These percussive, pianistic, and compositional innovations converge in Cage's music for prepared piano, the Music of Changes, and then oddly coalesce into the infamous 4'33". Conceived in 1947 or 48 and premiered by David Tudor in 1952, 4'33" was in fact not originally intended for piano. ${ }^{111}$ Nevertheless, since its premiere by David Tudor, the piece has remained associated with the instrument and, and has become dependent upon the presence of the piano for its effect. The physical presence of the piano is essential to the modern understanding of this iconic piece. Might it also be no coincidence that 4'33" was allegedly conceived at the same time that comedian and entertainer Jimmy Durante integrated total piano destruction into his stage act? ${ }^{112}$ Said Cage: "I didn't wish it to appear, even to me, as something easy to do or as a joke. I wanted to mean it utterly and be able to live with it." ${ }_{113}$ This is in seeming contrast to Durante, who certainly expected laughs. However, there is also something quite serious about Durante's act, just as there was something disturbing underlying his dismemberment of the English language. There is a presence of the deconstruction of tools of communication in service of something else. Is 4'33” really so different? It isn't it also a little funny?

\footnotetext{
111 4'33” was inspired by Robert Rauchenberg's white paintings of 1951, as quoted in a 1973 interview with Allan Gillmore and Roger Shattuck in Richard Kostelanetz, Conversing With Cage (New York: Limelight Editions, 1988), 71.

${ }^{112}$ LIFE Magazine, September 20, 1948, 14-15. See also Anthony DiFlorio, Jimmy Durante A Piano Is a Delicate Thing 44 70, accessed April 22, 2017, https://www .youtube.com/watch?v=sGiT-uJR4ZY.

${ }^{113}$ David Revill, The Roaring Silence: John Cage, a Life, 1st U.S. ed (New York: Arcade Pub, 1992$), 164$.
} 


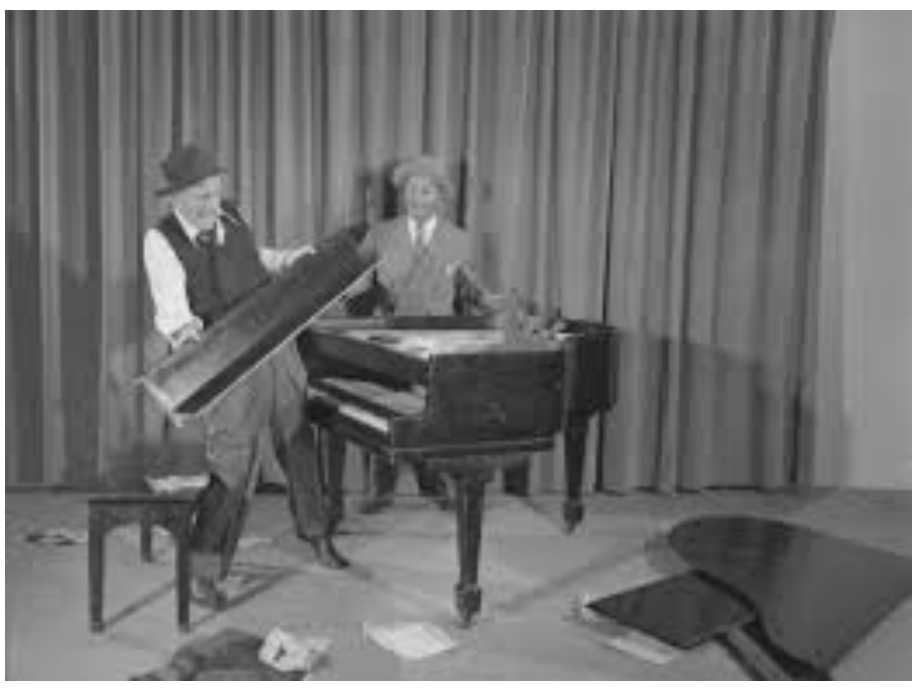

FiguRE 9: JIMMIE DURANTE IN PERFORMANCE ${ }^{114}$

From Ives and Ornstein, from Cowell to Cage and Durante, Busoni to Boulez, there is a gradual progression towards the outer limits of traditional piano technique and then, in the turn into the sixties, a substantial jump far beyond, forcing a confrontation with the very objecthood of the piano. Very little in these previous works, save perhaps Cage and Durante, prepare us for the radical, yet nuanced reevaluation of the instrument required by Ichiyanagi, the dialectic of post-bop piano and sheer choreographic physicality of Taylor's improvising, or the relentlessly extreme pianism demanded by Stockhausen.

In this transition into the 1960 s, the presence of the piano begins to be felt in a different way, and not only from within the compositional avant-garde. Composers, performers, and audiences seem to be reevaluating their relationship to this object. The cultural relationship with the piano in the late 1950s and early 1960s is clearly under duress. It is fractured, problematic, broken. Lines of communication are cut off between performer, instrument, and audience. For instance, consider this example below by Sylvano Bussotti from 1959:

\footnotetext{
${ }^{114}$ Image taken from LIFE magazine, September 20, 1948, 14-15.
} 


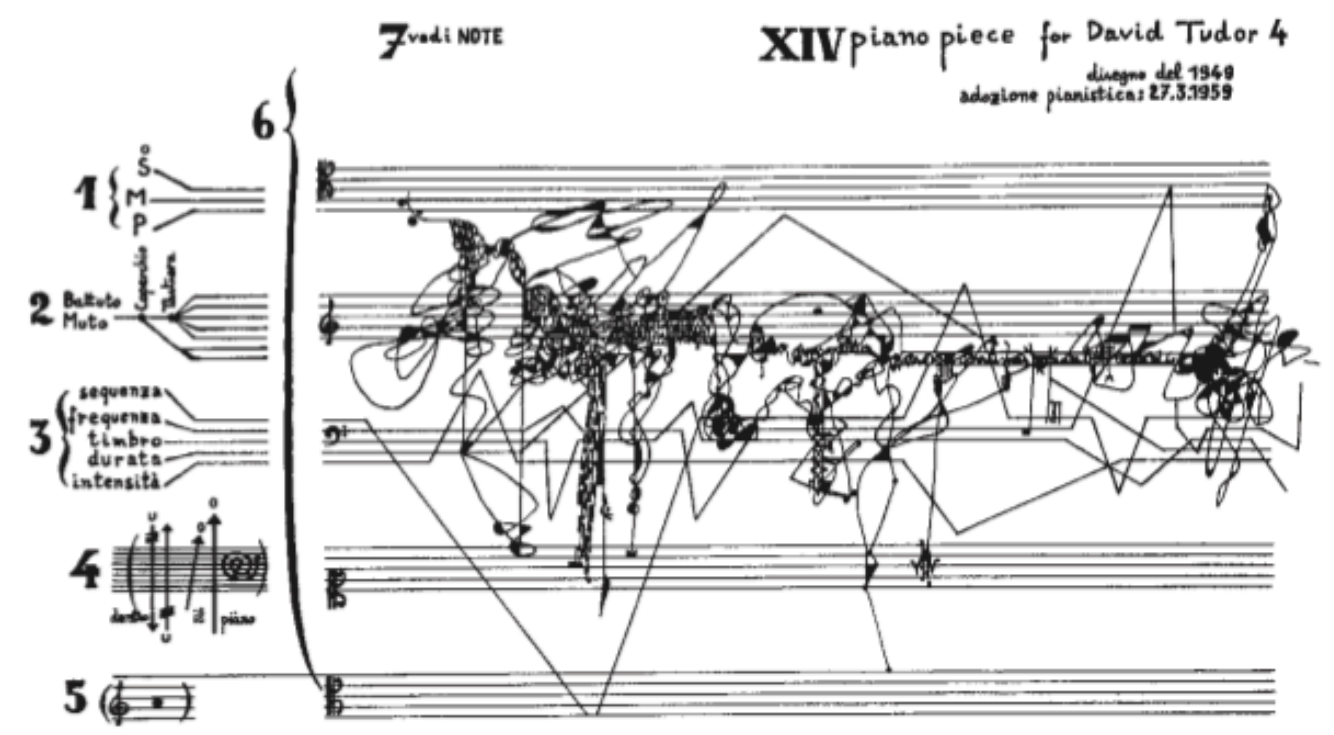

Figure 10: Opening of Sylvano BussotTi's Five PIECES For DAVID TUdor, Piece 4115

Such a work in one fell swoop deconstructs everything about the composer-notationperformer-instrument-audience-sound exchange. From this explosive moment of brokenness and disintegration (or-to use Deleuzian terminology - deterritorialization) there emerges a wealth of different practices focusing on different particular aspects of this deconstruction. Such work resides at a deconstructive nexus, representative of a comprehensive breakdown.

Such activities indeed were not limited to the musical avant-garde. In August 1961, the Music Trades Magazine published a report on an unusual fad on college campuses called "Piano Smashing." 116 It had its beginnings in England at the Derby and District College of Technology and eventually spread throughout other campuses in England and the United States. ${ }^{117}$ At Caltech, a Piano Reduction Study Group was

\footnotetext{
${ }^{115}$ Image taken from Ronald Bogue, "Scoring the Rhizome: Bussotti’s Musical Diagram," Deleuze Studies Volume 8, no. 4 (2014), 480.

${ }^{116}$ As quoted in LIFE magazine, March 8, 1963, 43-45.

${ }^{117}$ LIFE magazine, 43-45.
} 
organized, to reduce the piano, "in the shortest possible time, to such a state that is may be passed through an aperture of $20 \mathrm{~cm}$. in diameter," using only hammers that weighed less than 7 kilograms, or 15.4 pounds. In 1961, Caltech held the record time of 10 minutes and 44.4 seconds. ${ }^{118}$ Explained Caltech Piano Reducer Robert W. Diller, head of the team: "Piano reduction has psychological implications which are pretty dear to us. It's a satire on the obsolescence of today's society. We are sending out a brochure to see if we can get competition started all over the world. We'll start with the Paris Conservatory and the Julliard School of Music.” LIFE magazine reported: "the Caltech boys had nevertheless started something which could set relations between science and culture back 100 years." 119

\section{Piano Burning}

The long sixties are conveniently bookended by two very different, yet significant incidences of pianistic immolation: Jerry Lee Lewis' apocryphal piano burning of $1957^{120}$ and Annea Lockwood's “permanent preparation” of the piano 1968. ${ }^{121}$ These two extreme acts bracket an entire epoch in which new pianistic cartographies are mapped, reterritorializing the formerly staid landscape of wire and string. This is clearly a moment of reevaluation, of experimentation, and of struggle. Between these two events lies a decade that begins with radical questioning and exploration of all aspects of music,

\footnotetext{
${ }^{118}$ LIFE magazine, 43-45.

${ }^{119}$ LIFE magazine, 43-45.

${ }^{120}$ See for instance Robert Palmer, “The Devil and Jerry Lee Lewis,” Rolling Stone (December 13 1979), http://www.rollingstone.com/music/news/the-devil-and-jerry-lee-lewis-19791213. There is quite a bit of disagreement about whether this actually happened. See also Chris Heath in GQ, "The New Jerry Lee Lewis Biography is Definitely Undefinitive," (October 27, 2014), http://www.gq.com/story/the-new-jerrylee-lewis-biography-is-definitively-undefinitive.

${ }^{121}$ Other significant piano burners are Alejandro Jodorowsky, Yosuke Yamashita, Arman, Nam June Paik, and Chiharu Shiota.
} 
performance, and notation, often all at the same time.

Their differences, however, are telling. Jerry Lee Lewis' act (if it actually happened) was rebellious, performative. It was intended to upstage Chuck Berry, to assert dominance. The fact that he played while it was burning, while poetic, was ultimately just the icing on the cake. Lockwood's piano burning has a different resonance. It comes out of an era of burning effigies, American flags, and "the status quo" as Lockwood puts it. ${ }^{122}$ Central however to Lockwood is that the event had a sound and a performative presence, in addition to the appearance. She went so far as to wrap microphones in asbestos at the first performance. ${ }^{123}$ It was not only about theater. It was also a sonic event.

It was not the destruction which fascinated me. I am interested in something less predictable, arising from the gradual action of natural forces - fire, water, wind, plants, and earth - on an instrument designed for maximum control. I am interested in process. ${ }^{124}$

Lockwood's Piano Burning is different. It comes from a composer, form inside the compositional tradition. It was a process that relinked the piano to the outside world from which it came, to the realm of the non-human. It was a complex gesture and a terminus. The destruction of a grand piano has been interpreted as a gesture towards the deconstruction of the musical work and the cultural infrastructure that it represents. ${ }^{125}$ However, I suggest an interpretation that rests more on the reevaluation of the relationship between tool and user that opened up vast new musical possibilities. Through these experiments, aspects of the piano specifically the nature of musical instrumentality in general is gradually revealed. And, in a broader sense, such work asked challenging questions about instruments and instrumentality, which were both the fulfillment and

\footnotetext{
${ }^{122}$ Lockwood, How to Prepare a Piano, 21.

${ }^{123}$ Lockwood, How to Prepare a Piano, 20.

${ }^{124}$ Lockwood, How to Prepare a Piano, 21.

${ }^{125}$ Hans Belting, The Invisible Masterpiece (London: Reaktion, 2001), 394.
} 
destruction of the project of modernity, which largely rested on the rigid separation of subject and object.

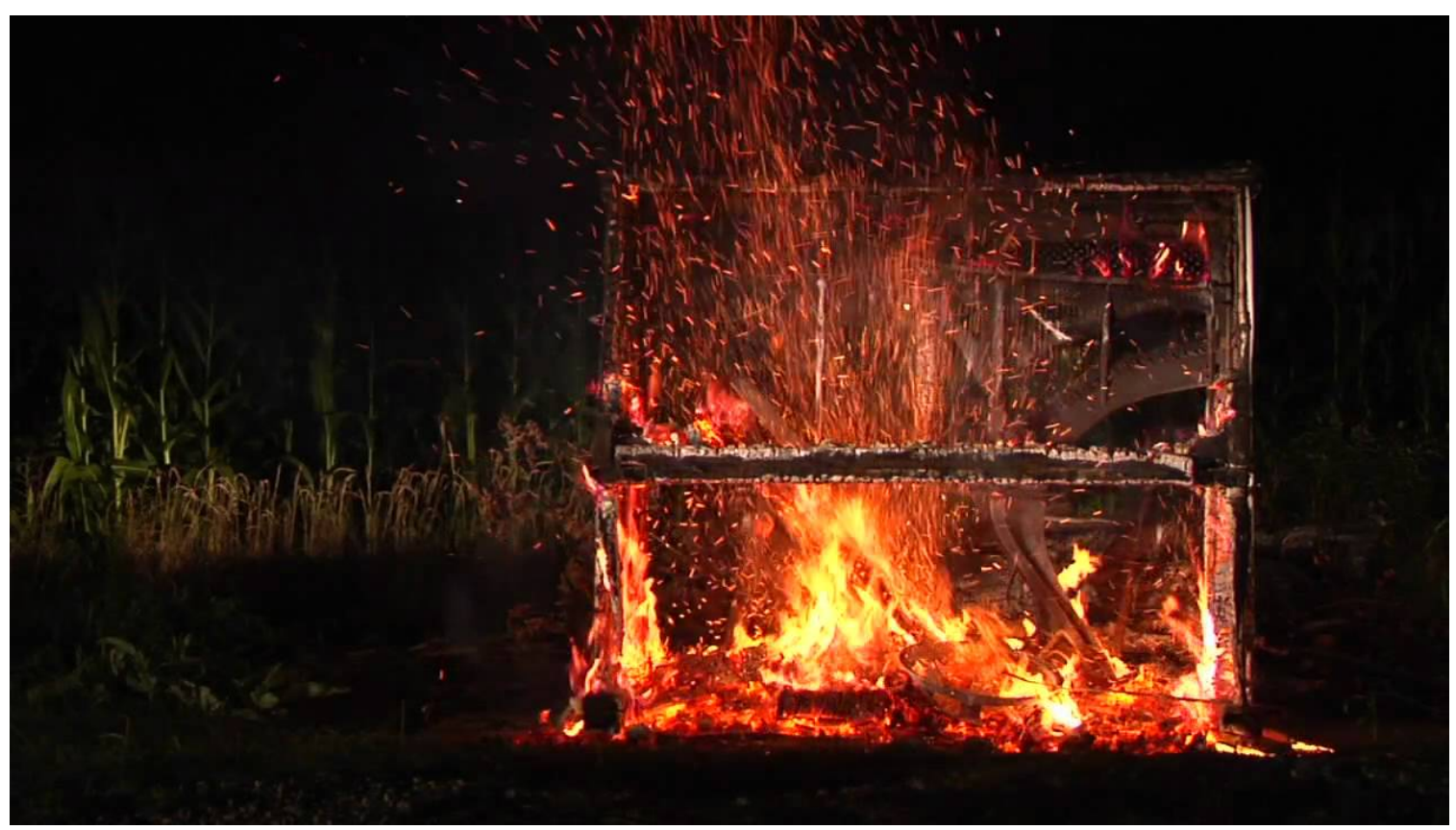

FIGURE 11: A BURNING PIANO. 126

Yet the musical instrument is no typical object. If we just examine the diversity of materials that make up musical instruments as opposed to say paintbrushes, the comparison seems quite uneven. There is also the physical complexity, design, and craft of most instruments; the cultural associations, the way they are of different sizes - some like toys, some similar in size to people, some are more like industrial machines, or like an organ, some merge with a room. Musical instruments have a distinct ontological presence within art making, due to their many variations and their presence in performative contexts, that makes them a very different sort of tool than a paintbrush (or

\footnotetext{
${ }^{126}$ Still taken from Michael Evans, Piano Burning (HD), accessed May 8, 2017, https://www.youtube.com/watch?v=WTyMmzqBjys.
} 
a chisel, chainsaw, arc-welder) on the one hand, and a computer (or staff paper) on the other. The tools of art can structure and entire discourse, an entire world. ${ }^{127}$ But in the case of instruments, the tools also point outwards to the world. A paintbrush, while certainly facilitating and constructing a world, tells me limited amount about, say the wood, metal, and squirrel hair that comprises it. However, with a cello, for instance, one can hear the evidence of the wood, the metal, the air, and friction in action. The effort, the forces, the evidence of the instrument's structure in the phenomenal moment is an important part of the instrument as tool.

That composers and musicians did not reflect upon the material developments of modernism as explicitly as in the world of the plastic arts is understandable. Composers and musicians for many years had preferred dwelling on either issues of expression or on specific and detailed elements of compositional structure. ${ }^{128}$ Music composition has typically been framed as what is written, as opposed to the actual material things, people, or processes making the music. Further complicating matters is the fact that embodied elements of live music have always been more phenomenologically present in ways that are typically obscured in traditional forms of painting and sculpture - scenarios where the maker's hand was often hidden. In addition, sound, in its production, perception, and its complex mechanics, can be difficult to understand or visualize. Phenomenologically, it lacks a degree of tangibility. Due to its performative live-ness and the endless contingencies regarding the division of musical labor, manifestations of musical practice are far more complex and difficult to detect than in conventional painting or sculpture. This is a result of complexity of human and non-human interrelationships in musical

\footnotetext{
${ }^{127}$ See Bernard Stiegler, Chapter 3.

${ }^{128}$ There are of course exceptions, normally in the realm of electronics or microtonal instruments, figures like Busoni or Russolo in Europe, and Varese or Partch in the United States.
} 
performance and the opacity of the final result.

\section{The Isolated Instrument}

So how did we arrive at this point of instrument as total configuration? One explanation for this development can be traced back to the dissolution of tonality leading to extreme rationalism and subsequent parameterization emerging form serialism's obsession with the isolated tone-the punkta. M. J. Grant in her book Serialist Aesthetics describes how the postwar European avant-garde searched for new sounds, timbres, and instruments on the one hand and strategies for restructuring musical composition as permutations of elementary structural units on the other. ${ }^{129}$ Grant suggests that this modern music (European concert music of the postwar years) had as its central figures both Webern and Debussy. ${ }^{130}$ This is representative of dialogue between tone-color/timbre on the one hand, and the ramifications of music that proceeds not from the scale or the triad, but the punkta, or "the isolated tone", on the other. Grant views this division as fundamental to both the acoustic and electronic within the powerful theoretical orbit of Darmstadt and the influential journal Die Reihe. This division is roughly comparable to the parallel history of electronic music, represented by the animating forces of musique concrète on the one side, and the parametric, structuralist approach of Elektronische Musik on the other. ${ }^{131}$ This drive in electronic music is accompanied by a rejection of the limitations of traditional instrumentation and results in an embrace of the aesthetic value of the sound itself. Ironically, is this very focus, intended to create a movement away from traditional

\footnotetext{
${ }^{129}$ M. J. Grant, Serial Music, Serial Aesthetics: Compositional Theory in Post-War Europe, Music in the Twentieth Century (New York: Cambridge University Press, 2001).

${ }^{130}$ Grant, Serial Music, Serial Aesthetics, 103-127.

${ }^{131}$ Grant, Serial Music, Serial Aesthetics, 55-57.
} 
instruments that in fact wound up encouraging new possibilities in the media they rejected..$^{132}$

These ideas achieve a synthesis in Schoenberg's concept of Klangfarbe. This is typically translated as timbre literally meaning tone-color, but Schoenberg's meaning is actually less clear-he uses the term to indicate not just a tone or an overall timbre but closer to something like a sound event, encompassing a the phenomenal moment. Schoenberg viewed timbre as a higher category of music, of which pitch is only one factor. ${ }^{133}$ In the non-electronic world, any Klangfarbe is intimately tied together with gesture and movement directed towards some instrument. As postwar serialism proceeded to parameterize pitch, rhythm, form, and timbre, we see a gradual emergence of something like the "isolated gesture." Complex gestural activities, movements, or techniques become parameterized and subsequently utilized in a generative fashion. This tendency begins in the late fifties and becomes clearly present in the mid-sixties to the early seventies.

Stockhausen was the first composer to truly fuse musique concrète and Elektronische Musik, as well as one of the first to explicitly build a musical language beginning with the punkta, building to the punktuelle. This contributes directly to the emergence of Sonorism and the influence of parametric compositional techniques. Early Fluxus composers like John Cage and Toshi Ichiyanagi begin with a similar parametric approach, though nothing quite as elaborate as Stockhausen's or Kagel's. Later Fluxus

\footnotetext{
132 "Instrumental music is redefined by electronic music: this leads to a previously unsuspected function for the instrumentalist. His characteristic criteria for the realisation of sound become serial factors in composition. The scale of degrees of approximation, corresponding to the mensural determinacy of the notation, become formative qualities." Karlheinz Stockhausen, "Introduction to the PIANO PIECES," taken from a printed supplement from the 1957 Darmstadt Summer Courses, trans. Richard Toop, April 4, 2014, http://web.archive.org/web/20140404172922/http://www.stockhausen.org/intro_piano_pieces.html. ${ }^{133}$ Arnold Schoenberg, Theory of Harmony (Berkeley: University of California Press, 1978), 421-422.
} 
composers take all of this a step further, but as a movement, Fluxus overall ultimately seemed to have little taste for explicit parameterization, preferring instead text scores, actions, happenings, and indeterminacy.

Simultaneously, there are other composers in the late 1950s, like Iannis Xenakis, who though unprecedented usage of parameterization and mathematical calculation, manage to create highly gestural types of engagement with traditional instruments by proxy (see the glissandi in Metastasis or the extreme choreography necessary to execute a piece like ST-4). The work of the Sonorist composers, exemplified in the early works of Krzysztof Penderecki, happen around the same period (the late 50s to early 1960s) but take a slightly different approach to the parameterization of instrumental materials. Working with a table of correspondences of primary materials - wood, metal, hair, leather-Penderecki developed a compositional style based on material juxtaposition. ${ }^{134}$ These are in evidence in his orchestral work Flouresceces. Though overshadowed by Threnody for the Victims of Hiroshima, Penderecki's Quartetto per Archi was a landmark work. Composed in 1959, it contains many notational innovations and string techniques that have become commonplace - measured seconds instead of free time, a mixture of specific and abstract symbols, moments of gestural, indeterminate pitch, and the invention of new symbols. All of these are significant. This is a result of the techniques of his composing style at the time that resulted in music that is essentially more on timbre, instrumental color, and masses of sound. Interestingly, this style was based on a materials-based approach where different classes of sound producing materials were put into a quasi-serialized structure. ${ }^{135}$ So, while this work is notationally important, its

\footnotetext{
134 Danuta Mirka, Sonoristic Structuralism of Krzysztof Penderecki (Music Academy in Katowice, 1997).

${ }^{135}$ Mirka, Sonic Structuralism.
} 
essential focus is less on action based notation and gesture and more on composing with blocks of timbre.

\section{Objekt Musik}

These factors contribute to the eventual emergence of what might be thought of as "the isolated instrument." Or, if this isolated tone is the German Punkta then perhaps the isolated instrument should be called the Objekt? The idea of the abstracted instrument, or the instrument becoming unmoored from its traditional context, and in that process displaying components of its objecthood. Subsequently, there is a foregrounding of instrumentality. In serialism, there is the implication of every element possessing a general equivalence. By creating the strict hierarchy of a series, it has the paradoxical effect of eliminating the more hierarchical tonal contexture and, inevitably, points towards the isolated tone. So too does the isolated instrument become the sum total of its sound-making capabilities.

An aesthetic emerges in the late 1950s that begins to treat all sounds that an instrument can make with equal significance, despite the intentions of the instrument's designers or the history of its technique. In the broader cultural sense, there are also major trends in parallel art forms that had a significant impact on post-war music. Visual art, performance, and dance were essential, especially Abstract Expressionism. The unbroken line from Dada and Duchamp, especially insofar as Ready-mades which birth the found-object tradition in visual art, influenced both Fluxus the work of the Judson dance theater. This Objekt Musik is an investigation into the possibilities of the isolated 
instrument sets the stage for the musical instrument as total configuration and instrumentality as a focus.

These developments in modernist art were reflections of the modernist project writ large. Recall Graham Harman's description of Latour's modernity as the impossible attempt to create a radical split between objective natural fact and arbitrary human perspective. ${ }^{136}$ There is among composers an acceptance of a distance between sound (objective natural fact) and meaning (arbitrary human perspective). However, this radical subject-object (music-sound) split winds up having the effect of revealing instruments physical objects - as having the potential for being the musical subject. The appearance of the isolated instrument and the total configuration represents a very literal example of this modernist split. It is especially ironic that it was Adorno that was often utilized in defense of such instrument-oriented practices of the sixties. When Adorno admonished composers to use the most "historically advanced state of their materials" in the 1950 s and 1960s composers mistakenly took this to mean the exploration of the instrument itself as a resource. As composer Brian Ferneyhough observes:

"The 'development of material' had thus curiously come to mean precisely the opposite of that which Adorno had been advocating, and the transformation of the instrument by means of its sonic deformation and alienation was to emerge as possibly the major defining innovative attribute governing thinking on the shaping of instrumentally induced sound in the 1960s." ${ }^{137}$

\section{Afrological Traditions}

Generally ignored in the narratives of experimental musical modernism are what George

\footnotetext{
${ }^{136}$ Harman, Prince of Networks, 31.

${ }^{137}$ Brian Ferneyhough, "Shaping Sound," in Sound, eds. Patricia Kruth and Henry Stobart, The Darwin College Lectures, 2000, 160.
} 
Lewis terms "Afrological" traditions. ${ }^{138}$ In cultural centers like New York City, Chicago, and Kansas City: not just the new sounds and rhythm of postwar industrial life and its emerging technologically aided soundscape but also the polyglot diversity of urban American life. This is especially true regarding the music of African Americans, whose musical priorities were often different than their Euro-American counterparts. Such music, like the early New Orleans jazz of Louis Armstrong and Jelly Roll Morton, often trafficked in the non-notatable elements of idiosyncratic virtuosity, subtleties of rhythm and articulation, and a prizing of individual instrumental approach, a finding your "sound."

Afrological approach is intimately linked with a radical re-evaluation of possibilities percussion in the early twentieth century. Ferneyhough also shrewdly observes that percussion and percussive thinking opens the door for many innovations to follow, both on the performer and listener side. Ferneyhough sees pieces like Edgard Varèse's Ionization as not only introducing a new sound world and engendering awareness of instrumental possibility but also as a radical outgrowth of motivic thinking. He views pieces like Varese's Ionisation as revolutionary in this regard, and as one of the central pillars of experimental modernism. Varèse's music proved a decisive influence on the subsequent percussion-oriented music of John Cage. Varèse's influence, along with Cowell's, opened up the possibility for not just new sounds, but for any sound, instrument, or object to be incorporated into a musical context. Cage postwar music represents a fusion of the expanded sound world of composers like Varèse and Cowell, integrated with the structuralist thinking engendered by serialism. This lineage of musical

\footnotetext{
${ }^{138}$ George Lewis, "Improvised Music after 1950: Afrological and Eurological Perspectives," in Audio Culture, eds. Christoph Cox and Daniel Warner (New York and London: Continuum, 2004), 274
} 
thought developed by Schoenberg and Anton Webern that results in the parametric deconstruction of all aspect of composition that emerged in the 1950s and became prevalent in the $1960 \mathrm{~s}$. The

The Afrological tradition considers performance, practice, instrumental research, and indeed the unique cognitive mapping that an improviser develops over time, especially with their instrument or instruments all form what we can think of "acts of composition." George Lewis discusses this in different terms, claiming that an Afrological notion of an improviser's "sound" is analogous to a composer's "style" and that this sound is inherently phenomenal in nature, as opposed to formalizable. ${ }^{139}$ Afrological systems are more associated with improvisation, unique performances, and "social instrumentality." ${ }^{140}$ Within this Afrological approach, the value of the instrument itself as the compositional frame, and its performative and cultural affordances increases exponentially. To accept this suggests a different way of thinking about the relationship between composition and instrumentality.

That such highly influential developments ultimately end up quickly allowing any object to be instrumentalized is no surprise. This goes back to Cage's Imaginary Landscapes series and as early as 1939 with the last one in 1952. An interesting example of such an explicit use of non-instrumental objects in a compositional context would be Cage's famous performance of "Water Walk" (1959) on the live television show "I've Got a Secret" in 1960. (The secret was that Cage was playing a musical composition). It is hard to imagine the implicit thesis of "Water Walk" as anything other than that all

\footnotetext{
${ }^{139}$ Lewis, "Improvised Music after 1950," 282.

${ }^{140}$ Lewis, "Improvised Music after 1950," 275.
} 
things, including household objects and elemental substances, like water or heat, can be repurposed and instrumentalized and used within a musical context.

Ferneyhough credits the rise of percussion to the "powerful influence of vernacular musics, particularly jazz" and the influence of the cosmopolitan, "urban" sounds of North America in the inter-war years. ${ }^{141}$ Ferneyhough is right to see this as an important factor pieces like Ionisation. In the case of Water Walk especially, the influence of vaudeville and American novelty composers and entertainers like Spike Jones is as undeniable as it is incongruous. In general, however, the influence of jazz and "vernacular musics" 142 as he calls them still goes largely uncredited in the Eurocentric narrative. Nevertheless, we can see clearly how this integration of any object into music recontextualizes the possibilities of conventional instruments and furthermore how traditional musical instruments become a field for different sorts of action.

\section{Technology}

There is also the extensive matter of such musics and their relationship to recording technology and the commercial music industry. Freed from the barrier of notation, in terms of instrumentality, these technological possibilities allowed a new type of secondary orality, a literacy of timbre and instrumental nuance to emerge. ${ }^{143}$ Significantly, recording technology allowed the means of dissemination of Afrological music, to be represented in non-live performance to listeners. Following in the wake of these

\footnotetext{
${ }^{141}$ Ferneyhough, "Shaping Sound," 154. The word "urban" is also typically interchangeable with the early twentieth century African-American community. I imagine it not chosen by accident by Ferneyhough.

${ }^{142}$ Ferneyhough, "Shaping Sound," 154.

${ }^{143}$ The term "secondary orality" is from Walter J. Ong, Orality and Literacy: 30th Anniversary Edition (Routledge, 2013), 11.
} 
Afrological musics, free jazz, rock, noise, become the eventual homes for this exploration.

Included within this is the impact of technologies such as amplification and other sound reinforcement that popular musics tended to use. This operates in conjunction with the massive impact of sound recording, which occurs outside of just the small world of musique concrète Ferneyhough mentions. Recording technology allowed genres and practices that depended on the phenomenological moment of performance to be better understood (like blues or gamelan music) or to flourish and be disseminated as texts (like jazz). Indeed, recording technology altered the fundamental definitions of music and sound. By ignoring the dialectical relationship between recording technology and jazz and popular music, the effects of technology in changing how sound is conceptualized are underestimated, how the "grain of the instrument" becomes valued and how instrumental specificity can be captured and become something developed in its own right. This is important even if there is not a direct relationship between vernacular, commercial and popular music on the one hand and music composition in the traditional sense on the other. Music technology, like photography and painting, allowed acoustic music to become more of itself instead of being beholden to previous paradigms. Technology produced an "other" that allowed us to see musical instruments for the sounding objects that they actually are.

It can also be argued that the roots of this run even deeper than just recording technology itself. Due to ideas like Fourier analysis and technologies of sound reproduction, combined with the lack of need for a resonating body, the anthropocentric definition of music is replaced by a hybridized one in which human capacities are already 
connected with technical media. ${ }^{144}$ Recording fundamentally redefines the creative potential of music, sound, and instruments. And, importantly for a materialist musical theory, musical logic is supplanted with physical logic. ${ }^{145}$ This includes the logic of physics and technology but in this new context, these are intimately tied to instruments and meaning. They produce what Friedrich Kittler calls the music of the Media Age, or “'Other music' ...a music which would no longer derive its power from alliances with the medium of language and its 'meanings'." ${ }^{146}$ These cultural and technological changes are intimately tied together. Music based on sound, which can take or leave semiotics, sets the stage for materialist musical practices more broadly.

\section{Noise}

As the twentieth century unfolds, there is an unprecedented proliferation of new musical instruments, musical technologies, sound making-devices, augmentative extensions of pre-existing instruments, and instrumentalized objects. There all of these developments can be considered types of noise in the Attalian sense. As Paul Hegarty notes in Noise/Music, all of these technological challenges to the sound world "recalled something that had been lost: that instruments are machines." ${ }_{147}$ Though they are often conceived of as vehicular machines for human ideas or feelings, in the strictest sense they are "physical machines for displacing air ...via a huge variety of material forms." ${ }^{148}$ It is only

\footnotetext{
${ }^{144}$ Melle Jan Kromhaut, “'Antennas Have Long Since Invaded Our Brains': Listening to the 'Other Music' in Friedrich Kittler," in Thresholds of Listening: Sound, Technics, Space, ed. Sander Van Maas (New York: Fordham University Press, 2015), 95-96. In this passage Kromhaut combines ideas from theorists Friedrich Kittler and Wolfgang Ernst regarding technology, instruments, sound, and music.

${ }^{145}$ Kromhout, “Antennas,” 99.

${ }^{146}$ Kittler quoted in Kromhaut, 103.

${ }^{147}$ Paul Hegarty, Noise/Music: A History (New York and London: Continuum, 2007), 27.

${ }^{148}$ Hegarty, Noise/Music, 27.
} 
when "synthetic" instruments come along that we began to see what traditional instruments actually are..$^{149}$

The gradual diminishment of instrumental mystique and the demotion of prestige and skill via the promotion of disruptive noise and the expansion of the musical to all sound have paradoxically brought about a renaissance of traditional musical instruments. We can hear the noise that had previously been obscured. Indeed, much extended technique can be interpreted as noise in the most complex sense: culturally (what is she doing that to that instrument?), acoustical (scratch tones, for instance, reveal the noise component of string playing that was hidden there all along), or motor noise (taking advantage of the inconsistency of the human body, as opposed to hiding from it). Once alternatives to conventional musical instruments emerge, many traditional instruments stop serving their previous functions. In that process, they begin to reveal more of what they inherently are. Post-modern, post-electronic music reinvigorates traditional instruments, now freed to reveal their formerly hidden noise, continuing with the discovery of reservoirs of noise within even the most traditional of instruments. It also has the interesting effect, similarly to tape music and early electronic music of foregrounding the body and the instrument. The body is displaced or gone altogether in musique concrète but is foregrounded through the possibility of its absence. The same is true for the "missing" musical instrument. This has the result of altering the status of instrumentality in such music. Now the speakers, the machines, and even the performance space itself take center stage.

\footnotetext{
${ }^{149}$ Hegarty, Noise/Music, 27.
} 


\section{We Have Never Been Modern}

The total configuration indicates a historical fissure representative of what we will see in the next chapter as Unzuhandenheit or un-readiness-to-hand. Something about musical instruments and their relationship to performers and audiences, especially in notated music, had fundamentally broken. It would be easy to suppose that this is just a function of artists chafing at the limitations of old instruments and therefore pushing them to their limits. Yet the longstanding interest in artistic practices that originates in this moment indicates something more significant than just rebellion. The isolated instrument, the total configuration as material, and the instrument itself becoming the subject matter of the work is evidence of a breakdown, resulting in a blurring of the subject-object distinction. This moment is a pure expression the paradox at the heart of modernism. This cultural breakdown can be seen as originating from modernism's simultaneous promotion of the materials of art to subject matter, while at the same time struggling with the inability of imagining hybrid actors.

Hybrid actors are an idea from Bruno Latour's. ${ }^{150}$ In this book, Latour posits the idea that true modernity would involve the ability to think beyond the subject-object and/or nature-culture binary and recognize the complex, interrelated nature of society and technology (or nature, politics, and discourse). To Latour, objects and subjects are primitive pincers with which to grasp reality. ${ }^{151}$ Such truly ecological thinking, according to Latour, that takes the reality of networks of human and non-human actants, including

\footnotetext{
${ }^{150}$ This term borrowed from Haenisch, "Materiality and Agency in Improvisation," 150, 155. Latour, in We Have Never Been Modern, calls these hybrid objects 'quasi-objects'. In many other places, he speaks of hybrids and hybrid networks. One could also just simply call such objects "actants" but I prefer hybrid actors when it concerns conceptualizing extremely complex entities like Nature, Culture, or the environment - things that are not typically referred to as objects.

${ }^{151}$ Bruno Latour, "Another Way to Compose the Modern World," (112th AAA Annual Meeting, Chicago, IL, 2013), 1-6.
} 
hybrid actors like musical instruments - into account, has never really existed, and indeed still eludes us. Thus, we have never been modern. Science and modernity are synonymous to Latour, modernity being the era where the separation between subject and object were the precondition for the practicing of science, while in practice their segregation facilitated their intermingling:

"By segregating the world of the non-human (science) from that of the human (politics) in theory, and undoing this separation in practice, the creation of quasi-objects and quasisubjects was facilitated. However, there was no theoretical base which situated these hybrids ontologically, as a result of which the modern thinker could not fathom them. These hybrids were never really acknowledged as such, but existed only as a mixture, as a derivation of the pure poles. Just because modernity has never observed her own principles of purity in practice, one could say that we have never been modern." ${ }^{152}$

In Wambach's analysis, Latour recognizes this paradoxical moment of change where the separation of subject and object led to their joining.

In contrast to hard science, social science, or philosophy (granting perhaps the very important exceptions of Heidegger, Merleau-Ponty, and anthropology), it is in the domain of art and music that such ideas - that we might provisionally call ecological ideas, in the broadest sense - seem to have found their first and fullest expression. The painting of Jackson Pollock is a representative example. For there is no Lavender Mist without the emergence the hybrid actor, the network of correspondences between house paint, paintbrush, hand, body, gravity, canvas, barn, art gallery, vision...on and on. Lavender Mist is at once the full expression of modernist art and the source of its undoing. Jackson Pollock is not painting nature; in his words, he is nature. He assumes his place as a hybrid actor in an assemblage of objects, almost none of which are nonsocial. Or, alternatively, all of which are "natural" somehow or other. As Pollock himself

\footnotetext{
${ }^{152}$ Judith Wambacq, "Subject-Object in Martin Heidegger, Bruno Latour and Manuel De Landa," Constant Verlag, accessed April 21, 2017, http://www.constantvzw.org/verlag/spip.php?article79\#.
} 
said in response to whether he worked from nature: "I am nature." 153 The inability to categorize the two is the point: the full hybridization of the objects in this assemblage is a sign of the success of the work.

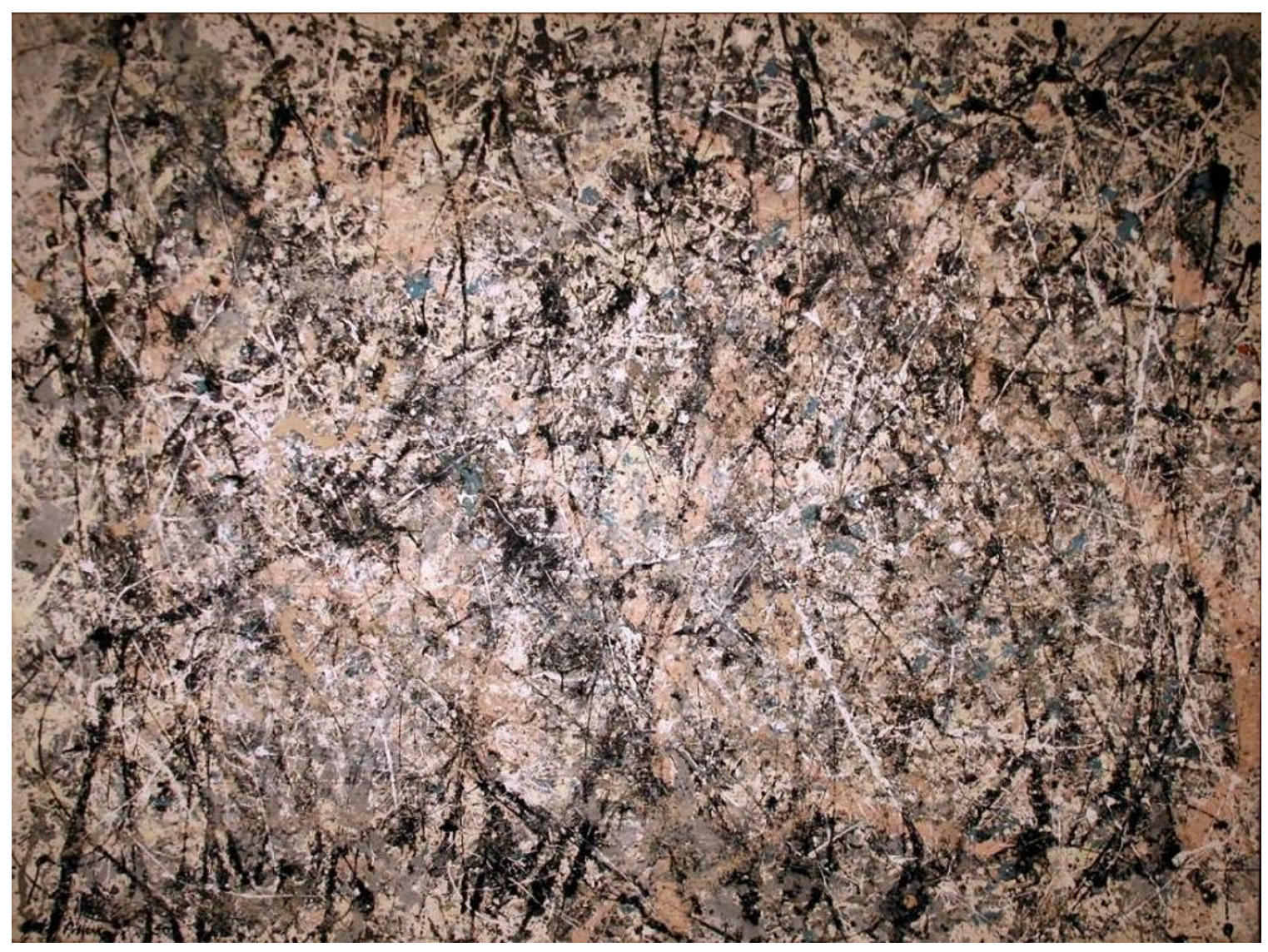

FiguRE 12: JACKSON POLLOCK'S LAVENDER Mist: NumBer 1, 1950 (1950).

One interpretation of Pollock's mature style is that the subject matter of the painting had become painting itself. However this was not exactly true: what is the subject matter of painting quickly becomes the process of painting, the tools of painting, the transference of action mirrored by the painting. The painting becomes a recording, evidence of a phenomenological confrontation. The substance, the paint, and the

${ }^{153}$ Attributed to Lee Krasner, "Oral History Interview with Lee Krasner, 1964 Nov. 2-1968 Apr. 11,” Text, accessed April 24, 2017, https://www.aaa.si.edu/collections/interviews/oral-history-interview-lee-krasner12507. 
processes become more and more significant. The performative triad here is analogous to music: action - instrument (tool) — sound (color/shape/drip, etc), and how they interact. The painting is a rendering of composite phenomenal moments.

In this development, the music of the turn into the sixties was not too far behind, if less self-consciously analyzed. Composition's confusion within itself between score, text, and music is somewhat to blame. But despite this, the instruments of music in this moment can be seen as asserting a different sort of presence posited on shifting paradigms of music and sound. This points to the importance of this work we are examining in the long sixties overall. In Benjamin Piekut's words: “The fundamental ontological shift that makes experimentalism as an achievement is that from representationalism to performativity." 154 If the main shift in experimentalism was from representation to performativity, then the value of instrumentality must increase accordingly. Instruments become one node in a hybrid network of physical, performative, and virtual spaces, of the types of hybrid actors/actants integral to art making. This is also a result of the "experimental sound ideal"; as defined by Judith Lochhead, the experimental sound ideal foregrounds a combination of science-style experimentation and a focus on the uniqueness of an event, or haecceity. ${ }^{155}$ Instrument-based practices provided just such phenomenal uniqueness. As we see in the seventies, improvised practices form ideal native territory for such music. But such an understanding of the materialist elements of the work was often rendered invisible through a combination of the conventional expectations of narrative and expression and the inherent

\footnotetext{
${ }^{154}$ Benjamin Piekut, Experimentalism Otherwise: The New York Avant-Garde and Its Limits, California Studies in 20th-Century Music (Berkeley: University of California Press, 2011), 7.

${ }^{155}$ Judith Lockhead, "Controlling Liberation: David Tudor and the 'Experimental' Sound Ideal" (The Art of David Tudor, Getty Research Institute, 2001), 1-6.
} 
phenomenological complexity of the sound making process. In Latour's thought, modernism, posited on a division of subject and object, in certain cases unwittingly embodied the synthesis of the human and non-human.

\section{Objects Become the Subject}

Latour's positing of hybrid actors and actants point towards a how to reimagine the subject-object dichotomy. American Experimentalism and European avant-gardism both open up conceptual space for the transformation of music into an assemblage of actants, of networks and feedback, recognizing hybrid actors as musical instruments integral to art making. In much African American music, this is just as true, or more. Yet both are problematic conceptually, as they are difficult to define from a materialist point of view. Sound, though a physical event, is invisible, ineffable; moreover, it is typically scientifically defined. Noise is by turns a scientific or social construct. Action, whose effects at least are somewhat clear in practice, is even more problematic in theory. The ontological status of sound, noise, and action are in flux even now and are often difficult to conceptualize. The far less theorized "instrument as total configuration" is a ubiquitous feature of the experimental and the avant-garde, which is still rarely theorized.

Within this already conceptually fraught environment of many experimental and avant-garde practices in the long 60s, the objects of art themselves become the implicit subject. Yet aversions or even antagonisms towards materialist interpretations of music obscure such developments. In music overall, reciprocal object-subject relations were not well understood. To imagine music in this way required a type of ecological thinking that 
did not yet exist, or that only existed in embryonic form. It was simultaneously created by modernism and invisible to it.

These forces combine into a nascent sort of "objectism" in music. This became an important area of work that engendered countless musical practices. Such musical objectism, even if it was not recognized as such, offered an alternative to the dominant aesthetic paradigms. And indeed has facilitated a number of musics that can be fruitfully interpreted as a phenomenological investigation into the relationship between instruments and the very act of music. Such Objekt Musik/objectism not only questions performative and compositional paradigms, but in so doing inevitably asks ontological questions about the nature of music and the nature of instrumentality. Through musics that are the result of collisions and translations, a network of asymmetrically relating objects, people, and environments in assemblages, there is recognition of contexture that ultimately points to an interrogation of the dominant subject-object relations.

\section{Action, Danger, New Cartographies}

One example of how instrumental object edges its way into the frame as the subject can be found in John Cage's Concert for Piano and Orchestra of 1957-58. This enigmatic, mostly graphic score contains sheet after sheet of unordered pages in what would to the uninitiated seem like inscrutable instructions. Most of these pages contain some sort of notational reference to staff notation-notes, note heads, staff lines, some sort of $x-y$ axis, interspersed with other elements. Then there is simply this: 


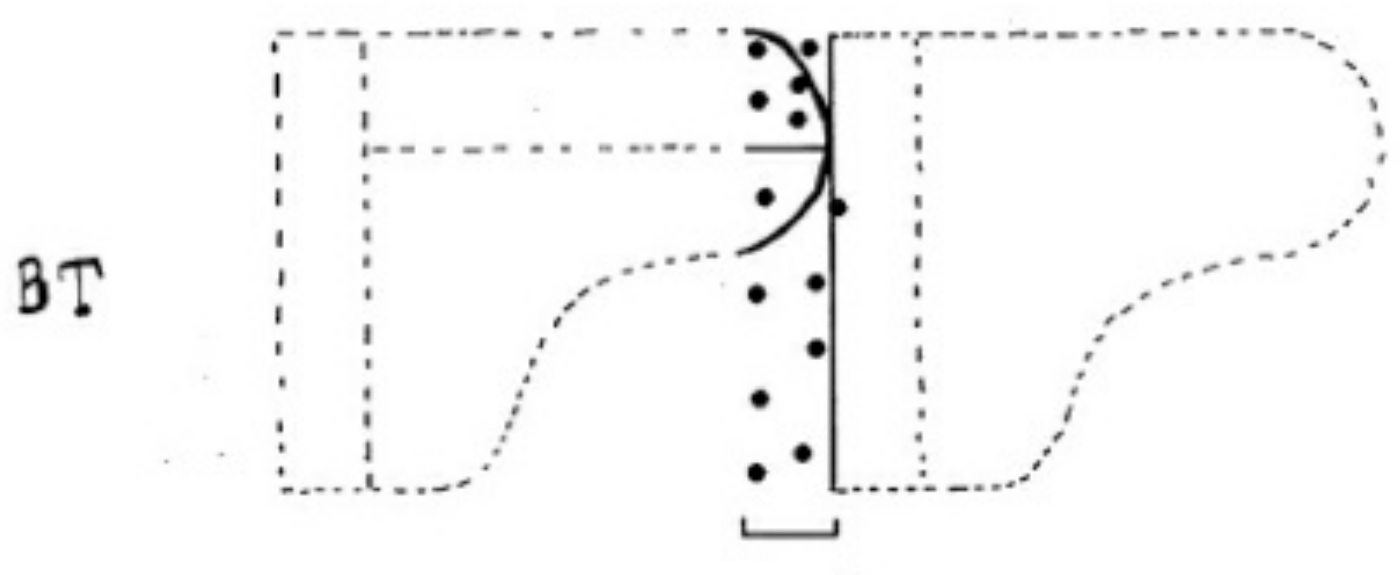

FigURE 13: EXCERPT FROM CAGE'S CONCERT FOR PIANO AND ORCHESTRA (1960) $)^{156}$

What is it is the performer is to make of this image? A request for engagement with the keyboard or body of a grand piano seems plausible. But why are there two of them? Should the piano be physically moved? Or is this a riff on the $x-y$ axis of conventional notation, indication of moving through time? And why does the end of one grand piano abut the other one?

As the long sixties continue, there are many permutations of this general theme, involving extreme extended technique, performance art, text scores, conceptual work, as well as into the realm of action, indeterminacy, and theater. In all of these, a musical instrument, or even more broadly the very concept of instrumentality, begins to play a central role. Ichiyanagi and Cage's fellow Fluxus traveller Lemonte Young for instance advocates a very muscular in his approach in his Piano Piece for Terry Riley \#1 of 1960. The instructions are simply:

Push the piano up to a wall and put the flat side flush against it.

\footnotetext{
${ }^{156}$ Image downloaded from http://www.newmusicbox.org/articles/an-audience-of-performers-part-1/ March 2017.
} 
Then continue pushing into the wall.

Push as hard as you can.

If the piano goes through the wall, keep pushing in the same direction regardless of new obstacles and continue to push as hard as you can whether the piano is stopped against an obstacle or moving. The piece is over when you are too exhausted to push any longer. ${ }^{157}$

It could be argued that Young's piano piece is certainly not the most sonically or conceptually interesting piece of his. Yet we should not be so quick to write off Young. In this piece, we witness another iteration of the instrument as an object, as an entityreflective both of what Heidegger would call its of its ontic presence (its presence within its context), which can also be interpreted as pointing outwards its ontological presence (its being in the greatest sense, its reality that lies beyond it). It creates radically new genres and practices, and ultimately has seismic effects on what music is and what it means. Like other object trouvé and found object art, such work interrogates the border between art and the outside world.

Often the instrument itself is all that ties the work to the tradition of musical performance or musical composition at all. An example is Fluxus founder George Maciunas' 12 Piano Compositions for Nam June Paik. Taking place in the context of works like Dick Higgins’ Danger Music series and George Brecht’s influential Piano piece No. 1 (1962) (“A Vase of Flowers on (to) a piano”), we see pieces that musicalize multiple aspects of a piano's existence, foregrounding its instrumentality..$^{158}$

\footnotetext{
${ }^{157}$ Taken from Mark Alburger, "Lemonte Young to 1960," 21st Century Music 10, no. 3 (n.d.), 9.

${ }^{158}$ James Martin Harding and John Rouse, Not the Other Avant-Garde: The Transnational Foundations of Avant-Garde Performance / C Ed. James M. Harding and John Rouse, Theater--Theory/text/performance (Ann Arbor: The University of Michigan Press, 2006), 227.
} 
12 PIANO COMPSITIONS FOR NAM JUNE PAIK, by George Maciunas, Jan.2,1962

Composition no.1 let piano movers carry piano into the stage

Composition no.2 tune the piano

Composition no.3 paint with orange paint patterns over piano

Composition no.4 with a straight stick the length of a keyboard sound all keys together

Composition no.5 place a dog or cat (or both) inside the piano and play Chopin

Composition no.6 stretch 3 highest strings with tuning key till they burst

Composition no.7 place one piano on top of another (one can be smaller)

Composition no.8 place piano upside down and put a vase with flowers over the sound box

Composition no.9 draw a picture of the piano so that the audience can see the picture

Composition no.10 write piano composition no.10' and show to audience the sign

Composition no.11 wash the piano, waz and polish it well

Composition no.12 let piano movers carry piano out of the stage

Figure 14: GEORGE MACIUNAS 12 PiAnO COMPOSITIONS FOR NAM JUNE PAIK (1960)159

Even more influential example is Philip Corner's Piano Activities (1962). ${ }^{160}$ A

performance in Germany in 1962 that resulted in the total destruction of the instrument

proved to be a defining moment for Fluxus, putting the comparative conservatism of the

European avant-garde into sharp relief. Corner was opposed to the highly destructive

interpretation of his piece in principle, which was more intended to get at hidden sounds

inside the instrument. ${ }^{161}$ Though he had reservations about the unintended level of

violence of the performance, he had to admit that the recording sounded pretty good.

\section{Tools Order a World}

In many cases, the instrument itself comes order the world of the music. Following the thread of the piano, we see cartographies of these transformations in Gordon Mumma's

MEDUIM SIZE MOGRAPH 1962. The score consists of choreographic notation (read

\footnotetext{
${ }^{159}$ Image downloaded from https://www.moma.org/collection/works/127428?locale=en, March 2017.

${ }^{160}$ For a contemporary performance of Piano Activities, see https://vimeo.com/111810763.

${ }^{161}$ Gunnar Schmidt, "Piano Activities," Piano Activities, translated from the German by Philip Corner, accessed April 24, 2017, http://piano-activities.de/englindex.html. The information on this website is a translation by Corner of a passage from the book by Gunnar Schmidt, Klavierzerstörungen in Kunst Und Popcultur (Bonn: Reimer, 2013).
} 
vertically) that provides a map of physical movement. ${ }^{162}$ Through the schematic representations of the piano frame and where to strike it, a different landscape of the piano emerges. The very act of mapping in such a way reorders this world (what we will see later as a 're-vealing' in the Heideggerian sense).

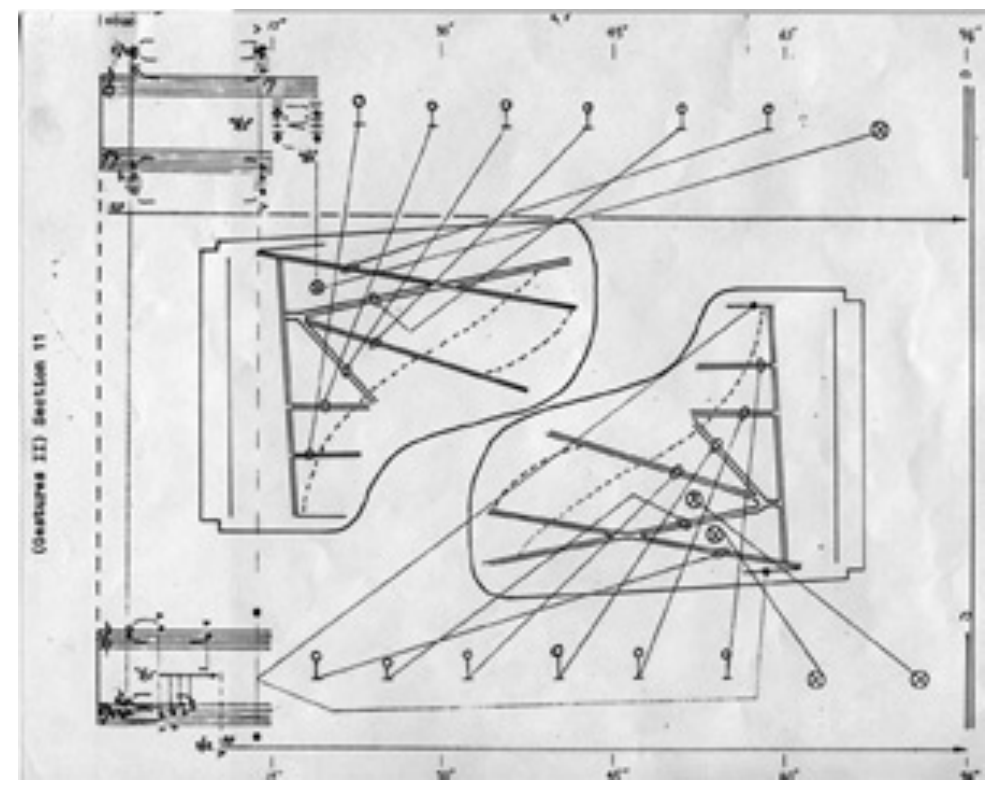

FIGURE 15: GORDON MUMMA'S MEDIUM SIZE MOGRAPH 1962.163

An extreme point on this continuum is Cornelius Cardew's Memories of You from 1964. Borrowing directly from Cage's Concert for Piano and Orchestra, the score is simply images of a grand piano shape from above with indications as to where to make a sound at, on, or near the instrument. ${ }^{164}$ This score is of course open to many sorts of interpretations. Whatever interpretation is chosen however it would be difficult to not engage with the tool-being of the instrument in some significant way.

\footnotetext{
${ }^{162}$ Gordon Mumma, "Gordon Mumma: MEDUIM SIZE MOGRAPH 1962," Leonardo Music Journal 21 (December 2011): 77-78.

${ }^{163}$ Downloaded from Mumma's website http://brainwashed.com/mumma/scores.htm, November 2016.

${ }^{164}$ Tony Harris, The Legacy of Cornelius Cardew, (Chapter 4 [no page])
} 


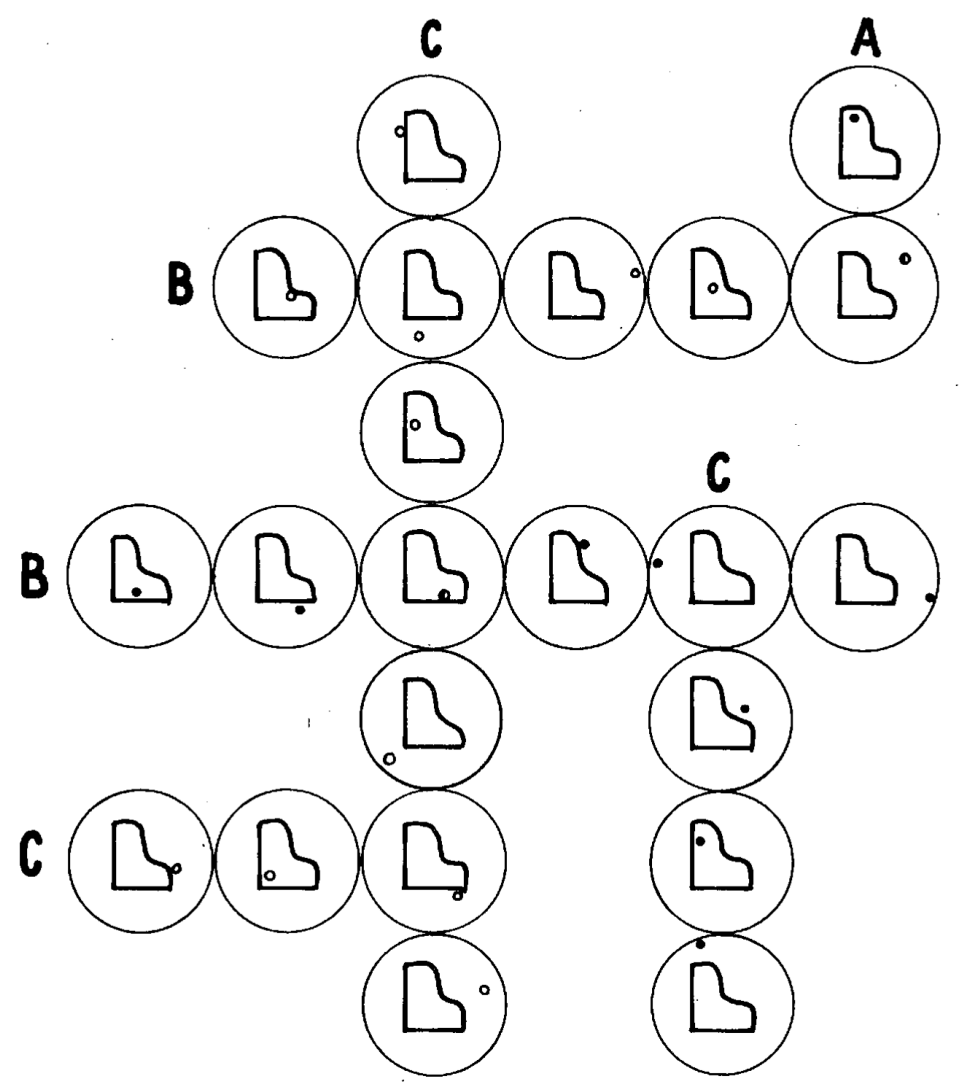

UE 14171 L

In all of these works we see how indeterminacy and experimentalism are instrumental in

${ }^{165}$ Cornelius Cardew, Memories of You: Piano Solo (London: Universal Edition, 1967). 
the transformation of instrumentality. These works change the very frame of what composition can be, of what composes the sounds. It integrates both performer and instrument into the compositional frame. It forms a trialectic by which notation, instrument, and composition all reveal new aspects of one another. From this emerges music rooted in the terroir of the instrumental ground, long denied its value.

In these pieces, we have reached a point far beyond extended technique. The presence of the piano as an object in a more complex sense emerges: its presence as tool, as machine, as industrial artifact; an awareness of the components that make it up, as wood, as furniture; its reality as a bourgeois symbol-all are felt in a significant way through their sonic traces. This is John Corbett's triple body, or the composite experiential musical body in action: the body of the performer, the body of the instrument, and the body of knowledge. All three bodies are involved here. It is the contribution of the instrument that is most difficult to apprehend however. As Latour reminds us over and over, we have a difficult time understanding the complex role that actants play in such a multi-dimensional pursuit as music. The sounds of object-oriented music are the results of an engagement with both the previously known and unknown properties of the thing. Something about the essence of the piano reveals itself in such a transformation. In works such as Ichiyanagi's, Taylor's, and Stockhausen's, the way the performer relates to the instrument must be redefined in order to even conceptualize how to perform the work. To play such music, one must reimagine the virtual instrument and what one's physical, emotional, and cultural relationship to it is. 


\section{Musique Informelle}

Adorno, our accidental prophet of Object Musik, advocates for a kind of music in his 1961 essay Vers une Musique Informelle that opposes the arbitrary parameterization and automatism of serialism through a fully integrated musical field that embraces spontaneity and expression. ${ }^{166}$ His reasoning, which has clearly evolved since his earlier Philosophy of New Music, comes out of an earnest attempt to appreciate the music of Boulez, Stockhausen, and Cage. In this essay, he spends a lot of time railing against the isolated tone as an inadequate basis for music but he does so largely on the basis that it does not provide enough resistance. ${ }^{167}$ However, Adorno grants that "themes" may not appear like "themes" in musique informelle. The central requirement is thematic material that, while not necessarily melodic and can be articulated at any level, has a theme-like force, creating a tension in specifically musical (as opposed to compositional) time..$^{168}$ Adorno will of course not concede anything like hybrid actors or actants. ${ }^{169}$ The result is a rejection of any work that operates in a more "ecological" mode or involves any form of distributed agency, either between multiple humans and/or humans and non-humans. As a result, he misses the potential in the ways in which movement, gesture, action, and technique, when applied to an instrument, can provide thematic coherence for music based less on pitch relationships or ungoverned by metric concerns. Though he clearly thinks that this type of timbre-based music is the music of the future (circa 1961) he does not have a good idea regarding how this would work. Having already cut himself off

\footnotetext{
${ }^{166}$ Theodor W Adorno, "Vers Une Musique Informelle," in Quasi Una Fantasia: Essays on Modern Music (London, New York: Verso, 1992): 269-322.

${ }^{167}$ Adorno, "Vers Une Musique Informelle," 298.

${ }^{168}$ Adorno, "Vers Une Musique Informelle," 314.

${ }^{169} \mathrm{He}$ also warns very specifically against the dangers of Die Aktion-Adorno, "Vers Une Musique Informelle," 314.
} 
from popular music in any form, Adorno does not see the forest for the trees that have already grown all around him. Indeed, it could be argued that Adorno was, in 1961, existing at the apex of the greatest period of "informal music" by his own definitionjazz-of the 20th century.

Even within the framework of notated composition, Adorno does not really have a good idea about what might come along to rein "themes" in, resistance that he accords as is essential. Which is what makes his ostensible dismissal of Objekt Musik even more confounding; it also makes Adorno's appropriation in service of exploring the total configuration more understandable. For, in the composite body of the performer/instrument, there are all the expressive vicissitudes and physics-based resistances necessary for a rich, musical language; but - and this is, of course, the central issue-composition must be displaced to contain it. For such music to exist, power and control — which he baldly articulates as dominion over nature-must be somehow ceded. Or, at least a collaborative relationship with the non-human must be developed. A more complex type of ecological thought must take hold. This is of course more than Adorno can accept.

This is perhaps why in the 1960 s, for the first time since perhaps the Renaissance, music composition (not music of course) fell behind visual art in theorizing its own relationship with its materials. On this playing field, visual art had the distinct advantage of being a physical object and having always been a more clearly detectable negotiation with the non-human. Mid-century visual art, even within its own critical practice, was ideally positioned to break down the subject-object dichotomy in a way music was not. However, this problem is not in the music itself, but rather in the appreciation of such 
practices, something that had more to do with critical discourse than perception or the music itself.

\section{Conclusion}

The interrogation of the subject-object relationship is what binds together the thinkers and the music enlisted for this project. This interrogation is also the basis for a concept of musical instrumentality. The central concerns of Latour and New Materialist thought find their theoretical origins in Heidegger and phenomenology. Latour that helps us see how these ideas can be used to understand and value our relationship with the non-human, indicating how complex and indissoluble it can be in practice. But it is Heidegger that gives us the basis to rethink the instrument itself - as both an object for philosophical analysis and as a tool for phenomenological exploration. 


\section{The Broken Tool}

"Equipment has a peculiar position intermediate between the thing and the work, assuming that such a calculated ordering of them is possible."

- Martin Heidegger ${ }^{170}$

\section{Un-readiness-to-hand}

Musical instrumentality forms the necessary conditions for music..$^{171}$ Despite this (or perhaps because of it) musical instruments typically remain withdrawn from us, in both theory and practice. Instruments and instrumentality often remain transparent, despite being right in front of us, or even resting in our hands. According to philosopher Martin Heidegger, this is not an odd state of affairs. In Being and Time, Heidegger develops a frequently referenced analogy of tool usage. In it, Heidegger claims that das Zeug - an untranslatable word, one often translated from the German as thing or tool, but also equipment (or even sometimes instrument ${ }^{172}$ ) - exists for us typically in one of two main

\footnotetext{
${ }^{170}$ Martin Heidegger, "The Origin of the Work of Art," in Poetry, Language, Thought, trans. Albert Hofstadter (New York: Harper \& Row, 1971), 29.

${ }^{171}$ This simplification leaves aside the ontologically complex cases of imagined, hallucinated, noncochlear, and/or the notated, the stockpiled, the potential, or other conceptual forms of music.

${ }^{172}$ As will be seen later, equipment and instrument have an interesting etymology: Middle English: from Old French, or from Latin instrumentum "equipment, implement," from the verb instruere "construct,
} 
states: zuhanden, translated as ready-to-hand ${ }^{173}$ and vorhanden, or present-at-hand..$^{174} 175$

Heidegger writes that when we are actively engaged in the use of a tool, we are not as concerned with the tool itself as an independent object; rather we are fully involved in the activity of using the tool towards some goal in the context of a project or process. ${ }^{176}$ When are engaged in using a tool and it is functioning properly, it is zuhanden, or ready-to-hand. Zuhandenheit-readiness-to-hand-strongly implies active use and physical engagement. We care about the job to be done. The tool becomes like an extension of us, so much so that we lose sight of what the tool actually is. In such scenarios, tools seem to disappear from our perception, as they are so enmeshed in contexture and intentions. Such is the case when we use a pen, a hammer, or a video game controller. The physical object, while not gone, often recedes so far into the background as to be almost invisible when the tool is working properly and in its intended context. The structure of the equipment, while not necessarily consciously understood or reflected upon, is nevertheless present in the kind of Umsicht or circumspection that can only take place in an active dealing with the world. In contrast, when something is reflected upon or thought about, it is vorhanden or present-at-hand. ${ }^{177}$ Vorhandenheit-presence-at-hand-is perception or understanding in a reflective state,

\footnotetext{
equip."

${ }^{173}$ Literally to/for/at/in/with/into/as/towards-hand/have/touch-ness .

${ }^{174}$ Literally in front-hand/have/touch - ness; available; looking; beholding.

175 The entire passage summarized here can be found in Martin Heidegger, Being and Time, trans. John Macquarrie and Edward Robinson, Harper Perennial Modern Thought (New York: Harper \& Row, 1962), 95-107. An extended passage continues on to relate these ideas to references, signs, and towards Heidegger's concept of Dasein continues until page 122. Here and thereafter I am also indebted to Jordan Bartee's analysis of the Broken Tool analogy in Ph.D. dissertation “Ontological Toys," Brown University, May 2014.

${ }^{176}$ Heidegger, Being and Time, 99-103, 121.

${ }^{177}$ Heidegger, Being and Time, 42-43, 71, 99-103, 121.
} 
or the state in which we are able to theorize about a thing a scientific manner. ${ }^{178}$

These types of perception, Vorhandenheit and Zuhandenheit are not separate: though Heidegger does not make this particularly explicit, they are two phenomenologically interrelated aspects of dealing with any tool or equipment. Part of the motivation for this division is a critique of Vorhandenheit as a way of knowing. Heidegger claims that this reflective knowledge is the primary mode of philosophical and scientific knowledge and came to dominate our understanding the world. By contrast, Zuhandenheit is a kind of perception that is not available to abstract thought or reflection alone. The way he puts it, action through a tool has its own kind of sight. ${ }^{179}$

Heidegger indicates that any knowledge that does not account for active use gives us an incomplete picture. This is similar to the idea of Gibsonian affordances (see chapter 4), where active use and engagement are essential defining features of a thing. In the case of affordances and Vorhandenheit, active use is not only about subjectivity. It is also about reciprocity. Pure Vohrhandenheit/presence-at-hand, in our daily lives at least, is difficult to achieve - we do not normally reflect on it for long; typically, if something is a subject of reflection it brings the tool back to our world of actions, a thing to be acted upon, albeit perhaps in a transformed fashion. ${ }^{180}$

If a tool is vorhanden, to Heidegger it is not in use. Furthermore, upon any consideration of that object, we are getting a bloodless and abstract version of a thingnot the thing at all, but a representation of it - a remembrance, or extrapolation, a mapping, bound to whatever modality we are using to think it. Yet when something is ready-to-hand, we are using it as a tool and experiencing it in a different way. Through

\footnotetext{
${ }^{178}$ Heidegger, Being and Time, 75.

${ }^{179}$ Heidegger, Being and Time, 99.

${ }^{180}$ Heidegger, Being and Time, 103.
} 
this use we are rendered unable to understand other aspects of its being. We are in essence blinded by the very success of the hybrid world that the tool creates. And, even at our most attentive, we at most perceive sense data from the tool that, while reflecting essential aspects of the being of the thing, only reveal limited aspects of its totality.

However, according to Heidegger, there is an intermediary stage that occurs when our activity is interrupted through equipmental malfunction or absence. This state which we most often encounter a broken tool Heidegger characterizes as Unzuhanandendeit, or un-readiness-to-hand. ${ }^{181}$ The broken tool asserts itself into our view, standing out as a particular individuated thing district from whatever role it had been playing in our prior activity ${ }^{182}$ These moments equipmental malfunction, absence, or obstinacy — of Unzuhandenheit or un-readiness-to-hand-reveal flashes of the hidden being of a tool. When a tool is broken, it is freed from its contexture and reference, and displays properties of not as it is used but as it actually is. It is in these moments, this brief window, that we can glimpse elements of the true being of something. ${ }^{183}$

Through this division, Heidegger manages to accomplish a number of things at once. First, he provides a basis for revaluation of everyday experiences and concernful dealings with the world and the things in it. ${ }^{184} \mathrm{He}$ also means to diminish the value of abstract thought and reflection, or at the very least show that it is inadequate. He wants to make clear that "objective," scientific, or even philosophical types of understanding in no way can give us a complete accounting of the being of something. ${ }^{185}$ Both modes of

\footnotetext{
${ }^{181}$ Heidegger, Being and Time, 102-104.

${ }^{182}$ Heidegger, Being and Time, 102-104.

${ }^{183}$ Heidegger lists three types of un-readiness-to-hand: conspicuousness, obtrusiveness, and obstinacy. See Heidegger, Being and Time, 102-104.

${ }^{184}$ Heidegger, Being and Time, 95.

${ }^{185}$ Or indeed of Being, keeping philosophers helpfully employed.
} 
perception are limited. But Heidegger also suggests something a bit more reciprocal: that there is a complexity of interrelations between subject and object, that the two are not as separate as we perhaps would believe. The misleading binary of the subject-object division can obscure the nature of a thing from us. Furthermore, he suggests that there are available states or amplified knowledge regarding das Zueg. It stands to reason that such knowledge gained through un-readiness-to-hand has these three characteristics: (1) it occurs in either of the two domains (the domain of reflection or the domain of use), (2) is reciprocal, and (3) is fleeting. However, it is in the gap, in the broken tool's un-readinessto-hand, that we are able to see radically new aspects of the object-evidence of unknown aspects of its being and of its contexture.

As the Broken Tool analogy shows, in attempting to further develop Husserl's phenomenological method to better accommodate experience, Heidegger manages to philosophically rediscover the object, the thing-in-itself. Furthermore, in drawing a more nuanced epistemological picture about how we deal with the world and the objects in it, Heidegger revives the then moribund idea of ontology. Important for musical instrumentality then is how Heidegger philosophically shows the multi-dimensional reservoirs of being that any tool possesses.

\section{Dis-closure, Re-vealing}

Heidegger's distinct variation on phenomenology shows how we access things in ways that can circumvent discourse and even conscious thought. This is part of a larger ontological project that seeks to draw connections between truth, art, and ultimately Being and Dasein. Heidegger's Broken Tool analogy is of a piece with his outlook on art 
in general. Heidegger sees the origin of the work of art as an exploration of this distance, this dissonance through revelatory type of disclosing. ${ }^{186}$ As Dermont Moran notes, Heidegger uses the Greek term for truth, aletheia, which has the etymological resonances of: “ 'dis-closing', 'un-covering', 'dis-covering', 're-vealing', that is: making manifest that which in some sense lies hidden.”187 By saying disclosure, Heidegger means that when I say something like "the hammer is heavy," the nature of the hammer is revealed in some way. ${ }^{188}$ Furthermore, that nature is something that cannot be grasped in any other way than through experience in the world. Reflection alone will not produce this revealing. Nor can discourse alone. Both have their place, mediated by moments of fracture.

Heidegger articulates this disclosing in terms less about the tools of the art than the subject of art, i.e. the shoes the painting represents, not the brushes or the paints that make the painting. The question of instrumentality that we are asking however involves inverting Heidegger's model: what is it that happens then when subject-object relation is reversed, and the tool itself becomes the musical subject? Or when, as Adorno says, we experience the disrelation of subject and object? It merely creates a situation of more regress, but not a different situation in kind. Indeed, music that explores such tool-being can point towards ways of understanding the deeper nature of das Zeug.

\footnotetext{
${ }^{186}$ See "The Origin of the Work of Art." There is also overlap to be found in his essay "The Question Regarding Technology" in which he develops a problematic analysis of more sophisticated technologies, which, as we will see below, Latour criticizes as a problematic misunderstanding of the complex and social infoldings of technology.

${ }^{187}$ Dermot Moran, Introduction to Phenomenology (London, New York: Routledge, 2000), 230.

${ }^{188}$ Moran, Introduction to Phenomenology, 230.
} 


\section{Tool-Being}

Philosopher Graham Harman brings a particular vision of Heidegger's work to bear that rests almost entirely on the tool-analysis above. In Harman's words:

Equipment in action operates in an inconspicuous usefulness, doing its work without our noticing it. When the tool fails, its unobtrusive quality is ruined. There occurs a jarring of reference, so that the tool becomes visible as what it is... ${ }^{189}$

Thus tools live a double life of action and disrepair. ${ }^{190}$ However Harman also states that no equipment can exist fully in one state or the other; or, put another way, that all equipment exists in both states at the same time, like poles on a continuum. ${ }^{191}$ The existence of any object is a relationship between these two states.

Harman's interpretation of Heidegger's philosophy can be reduced to one central idea: that there is an unbridgeable gulf between being and presence. To Harman, all presence is subjective and partial. Put another way, there is always a gap between experiential evidence of das Zeug and of the thing-in-itself, in which resides inaccessible reservoirs of being. The way Harman describes this is that when you sit at a table, there is the table that you sense and feel with your sensory experience. Then there is that table that you scientifically and theoretically understand is there, made of atoms, electrons, energy. Neither of these, however, are the actual table. The actual table, to Harman, is always withdrawn from all relations.

Object-oriented ontology (OOO) takes this very split as a point of departure for an entire branch of anti-correlationist philosophy posited on an ontology of objects. Taken to

\footnotetext{
${ }^{189}$ Graham Harman, Tool-Being: Heidegger and the Metaphysics of Objects (Open Court: Chicago and La Salle, IL, 2002), 45. He goes on to quote Heidegger: "The contexture of reference and thus the referential totality undergoes a distinctive disturbance which forces us to pause."

${ }^{190}$ Harman, Tool-Being, 45.

${ }^{191}$ Harman, Tool-Being, 45.
} 
this extreme, in $\mathrm{OOO}$ all objects are withdrawn from all relations with all other objects, instead creating caricatures of each other if they are recognized at all. All objects are in a constant state or caricaturing and misunderstanding each other's being. Heidegger distinguishes between pure presence-at-hand and the momentary recognition of a tool broken from its contexture/world. To Harman however, all presence-at-hand is ultimately a type of un-readiness-to-hand. All tools, when not inconspicuous, are ontologically broken in some way. Harman writes that Heidegger underestimated and underutilized this key insight. ${ }^{192}$ Harman states that the actual tool-being of an object is the reality of that object apart from any of its specific causal relations, sensed properties, and unexchangeable for any grand total of such relations. ${ }^{193}$ Harman believes Heidegger in fact did not fully understand the implications of what he had discovered, and spent the rest of his life dancing around this central insight, not comprehending the unity of Vorhandenheit and Unzuhandenheit.

The purpose of Harman's term tool-being is to indicate this ontological duality of all things, the unreachable being of all things, and any object's innumerable connections to the "empire of equipment." ${ }^{194}$ It points to the inherent ontological complexity of any tool or object. It is important to reiterate that we are typically blind to this tool-being. But, in exceptional moments we are made aware it. In this moment that we perceive flashes of the natures of being that are typically withdrawn from us. We only have occasional moments where we see true flashes of the true nature of something.

So without accepting Harman's object-oriented ontology in its totality, the concept of tool-being is useful, as it can encompass the full nature of the Heideggerian

\footnotetext{
${ }^{192}$ Harman, Tool-Being, 16-17.

${ }^{193}$ Harman, Tool-Being, 225.

${ }^{194}$ Harman, Tool-Being, 43.
} 
tool-usage analogy and more. It indicates any object's ultimate depth and un-knowability while indicating that it is inseparable from its contextual environment. This includes and the way it is made up indefinite levels of sub-objects and perhaps other tools within itself, and conversely its causal nature as it stretches out into the "empire of equipment" and beyond. My use of the term also seeks recognize the phenomenological engagement in the reciprocal between nature and culture that come to form it over time.

The elements of Harmanian tool-being that seem most important to instrumentality are:

1) Tools typically recede from us, in an inconspicuous usefulness; ${ }^{195}$

2) Their sensible façade is not what is primary about them; ${ }^{196}$

3) Tools are absorbed in the totality of the world, in the "empire of equipment"; 197

4) In spite of this, individual objects erupt into view: "forcing us to take stock of them, to settle our accounts with them." 198

It is important to note that to Heidegger, tools or equipment are not the same as objects. ${ }^{199}$ A tool or thing is to him a broader ontological category than an actual object. In his essay The Thing, Heidegger makes this very clear; he differentiates between jug-ness (das Zeug/the thing) and an actual jug (the physical object). ${ }^{200}$ Though of course the borders between the two are not exactly clear, he still finds it important to make the division. So another way of understanding Harman is to recognize that he believes Heidegger was wrong about this division. To Harman, there is no difference. This is important because Harman sees the difference between objects and tools as of "psychological as opposed to

\footnotetext{
${ }^{195}$ Harman, Tool-Being, 111.

${ }^{196}$ Harman, Tool-Being, 44.

${ }^{197}$ Harman, Tool-Being, 43.

${ }^{198}$ Harman, Tool-Being, 45.

${ }^{199}$ Bruno Latour, "Why has critique run out of steam?” in Critical Inquiry, 30 (Winter 2004), 233.

${ }^{200}$ Martin Heidegger, "The Thing," in Poetry, Language, Thought (New York: Harper \& Row, 1971$), 167$.
} 
ontological interest." ${ }^{201}$ Basically, if anthropocentric epistemology is de-privileged, we live in a subject-less universe of objects where everything is functionally equipmental to something else, even if it unbeknownst to any of the objects in question. This has the effect of drawing all things into an interrelated ontological field.

In order to build a concept of musical instrumentality, we have no need of such a totalizing ontology and all the problems it entails. We are in fact looking for something that is far more concerned with actual tools. But the ontological dimension is still necessary. Without that aspect, it is difficult to see how instrumentality points towards the outside world and to understand how music can be a form of phenomenological activity or investigation. The idea of un-readiness-to-hand is also important for understanding the mechanics of change, both culturally and individually. Therefore the concept of instrumentality I suggest resides at the intersection of these two visions. The border area between das Zeug as Heidegger envisions it and tool-being Harman envisions it is where musical instrumentality resides.

\section{Critiques}

\section{Avoidance of Simultaneity}

There are problematic elements in Heidegger's analogy in respect to aesthetics. These issues are subsequently multiplied in Harman's work. Both ignore of the possibility of multiple forms of simultaneous perception or awareness. For instance, philosopher Tom Leddy believes that Heidegger's and Harman's understandings of tools in Being and

\footnotetext{
${ }^{201}$ Harman, Tool-Being, 127.
} 
Time are deeply flawed:

Heidegger says that hammers are usually present to us only when they fail. This idea undercuts (and is in deep opposition to) the entire aesthetics of design. When I handle a hammer I am aware of its design. It is satisfying when it feels good in my hand, looks good, and does its job well. The awareness of the hammer I have when it is broken is different in kind from that: it is an awareness of a functional ugliness. But when a hammer works well, looks good, etc. it is a thing of beauty, more so of course for a carpenter than for me. Heidegger and Harman just exclude from consciousness the aesthetically positive value of the hammer. ${ }^{202}$

While denying the idea that the broken hammer must always display a functional ugliness, Leddy's general idea still holds from an experiential point of view. There are indeed multiple modes of perception of brokenness. These multiple modes can be experienced instantaneously. In fact, I can simultaneously: (1) use a tool (on the cello, I can think about phrasing as opposed to my bow and or hand); (2) be thinking about the cello as a theoretical entity and realize that somehow it is broken - both in (a) a broader social fashion (why am I making racket on this fragile, expensive instrument in this weird basement?); (b) contextually (I am not playing Schumann on this thing), or even (c) in a practical way (this string is broken!). In fact, is it not at all unusual to experience all of these thoughts at once, or in extremely rapid succession, especially in an improvised performance. Most performers can easily, and in fact are almost required to, consider the ontic nature (the domain ontology) of an instrument in action (how does this fit in with other instruments, within music or a style) and be cognizant of tradition and the role typically played, and balance accordingly. All of these elements are especially foregrounded in experimental or improvised music performance, largely as a result of how, as Keith Rowe says, the instrument is pointing outwards toward the world. This is

\footnotetext{
${ }^{202}$ Tom Leddy, “Aesthetics Today: Is Object-Oriented Ontology Good for Artists?” Aesthetics Today, August 6, 2015, http://aestheticstoday.blogspot.com/2015/08/is-object-oriented-ontology-good-for.html.
} 
problematic for Heidegger, Harman, and their acolytes. From an experiential point of view at least, their ontological divisions are far too primitive to accommodate aesthetic practice.

However, they do point towards a central component of instrumentality as opposed to equipmentality (like a microchip or a combustion engine) that might be more inconspicuous due to its structural complexity or intricacy. Instruments are usually utilized as a nexus of characteristics involving of multiple forms of complex perception on the part of the operator and, in the case of music, on the audience side too, though these are necessarily of different types. In the case of musical instrumentality, an extensive "inter-world" (to slightly misuse Merleau-Pontian terminology) is usually built, both personally and culturally, in order to operate the instrument. One could indeed argue that the complexity of simultaneous interactions and high skill levels that instruments typically demand is a core element of any type of instrumentality, in contrast to how we typically think of equipment or tool usage. Utilizing instruments usually involves precision, training, knowledge and a performative engagement. In the case of musical instruments specifically, this is especially true. And when they do not-like when a piano is set on fire-such an absence is a powerful part of its meaning.

\section{Depunctualization}

Another critique of Heidegger can be found in Latour's Pandora's Hope. Latour acknowledges Heidegger's important attempt to draw subject and object poles closer together ${ }^{203}$ However, Latour holds that Heidegger unwittingly preserves the fundamental

\footnotetext{
${ }^{203}$ Wambach, "Subject-Object in Heidegger, Latour, DeLanda."
} 
principals of the modernist gap. Heidegger privileges art and philosophy as the main avenues for understanding the interrelatedness of subject and object poles. ${ }^{204}$ Latour, on the other hand, regards the mixture of poles as a necessity for achieving a truly modern type of thought, one that was implied by modernism but was never fully achieved. Thinkers like Adorno on the other hand desperately attempt to retain this distance in direct opposition to Heidegger and especially in the case of aesthetics, art, and music.

Because of Latour's alternative analysis of modernity which demands a full accounting for the interrelatedness and hybridity of subject and object, he disagrees with Heidegger about the technology-tool distinction. In Pandora's Hope, Latour gives an excellent example through an example of un-readiness-to-hand, illustrating their phenomenal complexity and pointing towards the contextual, ontic, and ontological significance. He uses the example of an overhead projector. ${ }^{205}$ During a lecture, the projector appears to be a silent and mute intermediary, determined by its function. It is ready-to-hand. Suppose it then breaks down. We are now aware of the formerly invisible thing, since "the crisis reminds us of the projector's existence." 206 This is the moment of un-readiness-to-hand - of literal and contextual brokenness. But, this phenomenological jolt cannot last. Soon, the projector becomes a subject of further action-of care, of fixing, or repair. People are called, often materializing around the thing. Parts are ordered. In the worst case, it can even get broken down into further sets of sub-objects. The moment of un-readiness-to-hand in this circumstance is most likely quite brief.

Latour in this context is using this of an example of what he calls depunctualization or blackboxing. Blackboxing is a term to describe the practical way we

\footnotetext{
${ }^{204}$ Heidegger, "Origin of the Work of Art."

${ }^{205}$ Latour, Pandora's Hope, 183.

${ }^{206}$ Latour, Pandora's Hope, 183.
} 
ignore the true or hidden nature of a technology in order to focus on our usage.

Punctualization is similar to the idea of encapsulation in object-oriented programming. It is when the structure of the equipment locked away from view. Depunctualization then is the unearthing of what is inside of these walled-off objects. ${ }^{207}$ However, it is also a clear example of how a piece of equipment, technology, or instrumentalized object is a nexus of the social. It also revels how the projector is an actant in and of itself and has a degree of sociality. The object itself possesses instability.

A traditional musical instrument is no different. Cellos, pianos, Theremins, and synthesizers are many layers of instrumentalized materials. They are capable of literal and figurative breakage. They are also a social construct. Many of these layers are visible to us, and their properties are familiar to us. Yet many are not. Who in fact really understands how tungsten and steel are transformed in to a cello string? Or the Helmholtz regime that brings the bowed tone to life? Electronic or digital instruments are different in physical manifestation but ultimately not in kind.

Latour's main point of disagreement with Heidegger involves how in "The Question Concerning Technology" Heidegger was convinced that technology in general had usurped humanity, that it had mastered us. He contrasts complex technologies with more direct kinds tools that create a poesis that "ancient craftsmen" knew how to achieve. ${ }^{208}$ In this context, any piece of technology per se is too complex to be an instrument or a tool. But Latour sees this as an essential misunderstanding of the multilayered complexity of techniques and technology. Such tools, like overhead projectors or iPhones are merely multi-laminar, intertwined composite layers of human and non-

\footnotetext{
${ }^{207}$ Latour, Pandora's Hope, 183.

${ }^{208}$ As quoted in Latour, Pandora's Hope, 176. This type of language is indicative of problematic aspects of Heidegger regarding technology but in other areas.
} 
human activity. Latour argues that highly technological objects are ontologically no different from simple tools, just more complex. They are tools made of tools made of tools. This is the essence of punctualization. The overhead projector in the previous example is a many-layered object. Latour points out that the words 'technique' and its conceptual progeny 'technology' do not need to be used to separate human beings from the assemblies that they combine with, as the ontological nature of simple and complex technologies are the same. ${ }^{209}$ Technology is merely "congealed labor." ${ }^{210}$ Latour allows for a spectrum of the technical that that is alien to Heidegger. This spectrum of the technical is embedded in physical and non-physical locales alike. It is not easily locatable; nor is the social so easily banished from the object.

Latour is trying to break down the subject-object distinction to promote a more effective, less anthropocentric analysis of science. Harman by contrast is trying to develop a totalizing philosophical system through the revival of ontology. For understanding the evolution of instrumentality, Heidegger provides a basis for rethinking tools, whereas Latour provides a more practical way to discuss how this happens, and indeed how the breakdown of the subject-object dichotomy is an essential step for us to attempt and repair the damage of modernism and the correlationist gap.

\section{Techne, Technique, Technology; or Technology Reflects Us}

There are other theorists of tools and technology who build on these insights in different ways. One is the philosopher Bernard Stiegler, whose ideas about technology are beginning to become more widely known. A committed post-Derridian whose work

\footnotetext{
${ }^{209}$ Latour, Pandora's Hope, 191.

${ }^{210}$ Latour, Pandora's Hope, 189.
} 
builds on the thought of Gilbert Simondon, Stiegler is particularly interested in techne and technology. Though deeply indebted to Heidegger, he also critiques Heidegger for not going far enough in understanding the power of all tools and all instruments.

Not only does Heidegger think the instrument; he thinks on the basis of it. Yet he does not think it fully through: he fails to see the instrument in the originary and originarily-deficient horizon of any discovery, including the unforeseen; [...] He thinks tools as (merely useful) and instruments (merely) as tools, and he is as a result incapable of thinking, for example an artistic instrument as something that orders a world. ${ }^{211}$

Stiegler also states, rather poetically, that the instrument is like a mirror of anticipation:

...the place of recording and inscription but also a surface of reflection, the reflection that time is as if the human were reading and linking this future in the technical. ${ }^{212}$

Any tool, instrument, or technology is to Stiegler an inscribing of time. To Latour, tools and technology, from simple to complex, are a manifestation of all the forces that formed them: work, thought, accidents, consciousness, the environment - everything that went into their existence. Stiegler inverts this relationship, seeing them not merely as result but as the precursor of thought. Tools are a feedback loop of creation that preserve ideas in physical form, that inscribe time and work, but also create new forms of consciousness. They are time machines that manifest ideas from the past into the present while also collaborating in creating and structuring our future. Stiegler indeed develops a theory of technology in which tools and tool usage form the basis for thought, as opposed to the other way around. We see a different version of this in Heidegger, as seen in his essay The Thing. In an analysis of The Thing, Eugene T. Gendlin states, apocalyptically:

"Not only might man blow up the world with technology, technology has already gone far toward making man its appendage, making man into a thing whose

\footnotetext{
${ }^{211}$ Bernard Stiegler, Technics and Time I: The Fault of Epimetheus, trans. Richard Beardsworth and George Collins (Stanford: Stanford University Press, 1998), 244-245.

${ }^{212}$ Stiegler, Technics and Time I, 153-154.
} 
nature can say only yes or no within the structuring of technological projecting. The danger is man (and being) as made! ${ }^{213}$

However Heidegger more suspicious of advanced technologies, like computers and nuclear bombs, and not the humble cobbler's hammer or Van Gogh's pair of shoes to use two of Heidegger's favorite examples. ${ }^{214}$ Stiegler, like Latour, takes part of this argument (we do not create technologies, they create us) but leaves behind the other part (computers and hammers are ontologically differentiated). Latour and Stiegler seek to diminish the difference between technologies as just elaborate types of layering assemblages acting within other assemblages. Technique, techne, and the technicalLatour points out they all originate from the same etymology, from the same root. ${ }^{215}$ This creates an interrelated continuum, a spectrum of instrumentality, from the physical to the non-physical, the cultural to the natural, of technique/techne/technology. Musical instruments, especially conventional ones — like all technology — are "society made durable.."216 They are natureculture hybrids. Echoing Stiegler and Latour, Matthias Haenisch says of musical instruments that they: "stabilize the aesthetic and social relationships between musicians in that they preserve collective memories of experiences and keep them available for upgrades and advancements."217

\footnotetext{
${ }^{213}$ Eugine T. Gendlin, An analysis of What is a thing? In Martin Heidegger, What is a thing? Trans. W.B. Barton and V. Deutsch (Chicago: Henry Regnery, 1967), 247-296. From http://www.focusing.org/gendlin/docs/gol_2041.html

${ }^{214}$ Though to be fair to Heidegger, he does observe that science has managed to annihilate all things, and that long before the atom bomb: "The bomb's explosion is only the grossest of all gross confirmations of the long-since-accomplished annihilation of the thing: the confirmation that the thing as a thing remains nil. The thingness of the thing remains concealed, forgotten. The nature of the thing never comes to light, never gets a hearing." From Heidegger, The Thing, 170.

${ }^{215}$ Latour, Pandora's Hope, 190-191.

${ }^{216}$ Bruno Latour, "Technology Is Society Made Durable," in A Sociology of Monsters: Essays on Power, Technology and Domination, ed. J Law, Sociological Review Monograph No. 38, 1991, 103-32, http://www.bruno-latour.fr/node/263

${ }^{217}$ Matthias Haenisch, "Materiality and Agency in Improvisation," 164.
} 


\section{Withdrawal}

Any instrument, musical or otherwise, has a totality that, while doubtless real, is withdrawn from us, and indeed withdrawn from every other thing, each in a different way. (In fact, Harman defines the Real as that which cannot be perceived through the senses.) Merleau-Ponty compares this to a house, of which we can only see one angle at a time, slowly forming an aggregate picture. ${ }^{218}$ And, even if we could see all angles of the house at once, we would still only see it from our very limited human perspective. We can never see the house itself. It would always be a caricature. There is a totality of any thing that is withdrawn from us as humans, and from everything else for that matter. We are always already unable to recognize the full tool-being and contexture. To Harman the difference between present-at-hand and ready-to-hand is ultimately irrelevant. Both are different types of anthropocentric caricatures of the real thing. But, this caricaturing is not only limited to humans. All things are constantly mistranslating in their own ways tiny slivers of any other thing, blind to the true nature of any other thing. In Harman's assessment, this essential failure is at the very heart of all rationality. We can never grasp things as they are.

This points to why Harman's integration of aesthetics into his philosophy is unconvincing. Harman's commitment to the idea that all objects are withdrawn from all relations results in a one-dimensional view of relationality not representative of the multilaminar, multi-modal nature of human experience. If this is true in the special case of human consciousness, it is hard to imagine it can accommodate the far more complex

\footnotetext{
${ }^{218}$ Maurice Merleau-Ponty, Phenomenology of Perception, trans. David A. Landes (London, New York: Routledge, 2012), 69/95.
} 
cases of how objects or things in the world might be relating to other objects or things. However it is also simply true that understanding more and more aspects of something is possible, however ultimately infinite or difficult to ascertain such relations might be. We can think of art, and especially objectism in music, as an important way to get incrementally closer to the tool-being of a thing. However, we can certainly grasp more of thing. In this attempt, the moment of un-readiness-to-hand is key. A tool, in its brokenness, can show us more and more of its depths of being, extending outwards in every direction (materially, culturally, and historically) without ever actually exhausting the object at all. We can attempt to understand its own interior relations and its contexture, in all types of new and unexpected ways.

This type of exploration is one of the essential modalities of art. One way that this can be accomplished is via phenomenological engagement and ontico-ontological considerations of instruments and instrumentality. Such a reading of experimental practices is facilitated by ideas regarding distributed agency and assemblages and a willingness to break down the human/non-human division. We are able to take a different measure of both the tool and the contexture that it is enmeshed within. This happens most clearly when we perceive the tool as broken in the moment of brokenness, of unreadiness-to-hand.

\section{The Sound of Brokenness}

The reason this excursion into Heidegger has been three-fold. The first to argue that musical instruments in the long sixties, and in many practices thereafter either display, foreground, or cultivate un-ready-to-handedness in a broad and multileveled sense. The 
relationship of the musical instrument to music, to notation, to the performer, to societyall were in flux in the turn into the sixties, indicative of some sort of cultural brokenness. The emergence of instrumentality in many experimental practices thereafter, displays unready-to-handedness, both in an individual and broader cultural sense. But this is not a hopeless situation. For recognizing this brokenness provides an opportunity for a greater depth of understanding. The moment of breakdown was also the birth of a new moment of awareness. This simultaneously points to a breakdown in instruments' contexts but also to new insights into their true nature and possibilities, their tool-being. In the previous chapter, we see the piano become culturally unmoored, in a state of literal, figurative, and perhaps ontological brokenness. As we will see in the next chapter, this brokenness is also an opportunity for reflection and even reinvention.

Second, Heidegger's philosophy is essential to understanding the revival of ontological thought, which points towards the recognition of contexture that ultimately sets the stage for an interrogation of the dominant subject-object relations, resulting from a transformation of Hussurlian phenomenology. Thus Heidegger's ideas might be used to form the basis of rethinking instruments and instrumentality.

Third, I submit that many contemporary compositional practices consist of trying to foreground and cultivate this unsustainable moment of un-readiness-to-hand, practices that seek a constant rejuvenation of foreignness, of the result of a dialogue between elements of the composite body. In music, through a focus on phenomenological engagement with an object or traditional instrument, new or hidden aspects of an instrument's tool-being can be discovered. This can also be read as an ontological investigation into the interrelated aspects of nature and culture, and a new consideration 
of instrumentality within composition. Framed in a different way, such music can also model ecological thought. This process of discovery forms a basis for new musical practices. However, the criticisms of Heidegger should not be ignored.

As we have already seen, there is a pivotal moment at the turn into the sixties where the apex of cultural modernism and musical modernism coincide-where instrumentality was revealed in a new way through new awareness of instrumental contexture. These states of brokenness both revealed and required new cartographies, in both the more literal sense of musical notation but also in performance and recording. As composers develop practices native to this brokenness, a genuine practice of Harman's promised but never delivered "carnal phenomenology" is achieved, with musical instruments providing the basis. Experimental and avant-garde practices of the long sixties create the bedrock on which many contemporary practices still thrive.

The Broken Tool analogy provides a basis for a more nuanced understanding of instrumentality overall. Within such a framework, we can consider music as manifestations in sound that reveal the nature of a thing through performance, representations (notation), and re-presentations (recording). Literal (notated) and figurative (sonic) cartographies point towards to altered phenomenal relationships to the musical instrument itself, and to the assemblage in which it resides — disclosing in our case a different knowledge regarding the actual physical tools of music, but also the materialist elements of action and sound, and indeed any element the composite body.

The concept of Unzuhandenheit, despite its faults ontologically, provides a basis for understanding how we deal with the tools of music. Many experimental practices foreground just such a confrontations through attempts at retaining this very brokenness. 
This, of course, indicates failure in one sense but a failure allows us the ability to see different facets of what the instrument actually is and can be. Such new cartographies are a record of deconstruction and but also a map of new possibilities. Heidegger's ideas form the basis of a rethinking of instruments and instrumentality, but can also point towards contemporary compositional practices that foreground and cultivate this unsustainable moment of un-readiness-to-hand as an artistic method. 


\section{Instruments in the Expanded Field}

\section{Defining Musical Instruments}

Heidegger provides an analysis of tools, Harman a concept of tool-being, Latour of actants and hybrid actors. These ideas give us a framework for considering instruments both ontically and ontologically through the considering of the significance of tools, things, and the "empire of equipment." A concept of instrumentality must accommodate all these and more. Bernard Stiegler brings us a step closer an instrumentality, though instrument to him still seems equivalent to equipment and perhaps even tool. However, none of them deal specifically with musical instruments theoretically, much less in compositional practice. Any consideration of instrumentality for our purposes must be more specific, and one of musical instrumentality even more so.

Considerations of musical instrumentality are rare. ${ }^{219}$ Indeed, musical instruments themselves are rarely explicitly defined or defined poorly. ${ }^{220}$ The word itself comes from Middle English via Old French, or from Latin instrumentum "equipment, implement" from the verb instruere "construct, equip." ${ }^{221}$ A typical contemporary definition would be

\footnotetext{
${ }^{219}$ Sarah Hardjowirogo, "Instrumentality. On the Construction of Musical Identity." In Musical Instruments in the $21^{\text {st }}$ Century: Identities, Configurations, Practices, eds. Till Bovermann et al. (Singapore: Springer Singapore, 2017), 2-14.

${ }^{220}$ In Tellef Kvifte, "What Is a Musical Instrument?" Svensk Tidskrift För Musikforskning 90, no. 1 (2008): $45-46$.

${ }^{221}$ Oxford Dictionaries / English, s.v. "instrument," accessed March 12, 2017, https://en.oxforddictionaries.com/definition/us/instrument.This recalls Latour's equivalence of techne,
} 
"a technical construction for the purpose of music." ${ }^{222}$ Such a definition, while avoiding a difficult issue (what is music exactly?) partially accommodates something significant: that musical instruments are objects that, while having some cultural stability, can also be remapped, repurposed, reimagined, abrogated, or invented. Helpfully, this definition incorporates the idea that design and history play an important part, or at least a role, in most musical instruments. It is problematic, as it strongly implies that that a musical instrument requires being constructed with the purpose of music in mind. For many contemporary practices, this simply is not the case. Many contemporary practices involve an assemblage of elements, many of which were never intended to be instrumentalized for music. Or what of the voice? Though it might involve technique, I doubt that is what is meant by "technical construction."

Alternatively, the Oxford Dictionary of Music defines musical instruments as "Objects or devices for producing musical sound by mechanical energy or electrical impulses." ${ }^{223}$ This definition cleverly avoids the above problem through use of the present participle, putting the emphasis on the making of sound, as opposed to the making or design of the instrument. However, in common usage, to call something a musical instrument generally describes something far more specific than just any instrumentalized object. Instruments intersect with bodies of knowledge. Musical instruments usually have an intertwined design intent and history. They are usually a traditional part of a culture and/or purpose made. This is opposed to say "using a radio as an instrument" or

technique, and technology, which he implies as a continuum from the physical to the mental to the imagined, in which all modes of the technical exist.

${ }^{222}$ Paul Craenen, Composing under the Skin: The Music-Making Body at the Composer's Desk (Leuven: Leuven Univsersity Press, 2014).

${ }^{223}$ Michael Kennedy, ed., Oxford Concise Dictionary of Music (Oxford, New York: Oxford University Press, 2004), 359. 
instrumentalizing a radio. There are different shades of meaning in each of these activities - different cultural resonances, different compositional implications. Being used as an instrument is ontologically not the same as being a musical instrument, though such practices are connected by a shared sense of instrumentality. ${ }^{224}$ The definition of musical instruments must also encompass this gesture of instrumentalization.

In the broadest sense, a musical instrument can be thought of as on object that translates action in physical time and space to musical time and space. ${ }^{225}$ This definition is compelling but problematic in application to more ontologically complex cases. For instance, are software instruments considered objects? And if so, what is the nature of their physical or temporal transformations? Within a similar but more philosophical vein, composer and theorist Brian Hulse considers instruments within the framework (and distinctive terminology) of philosophers Gilles Deleuze and Felix Guitarri, writing: "Musical instruments are modulators of musical practices and their transmission" which produce deterritorialized lines of flight, conducting their own kind of flow and flux, "repeating, spreading, transforming thought in space and time." ${ }^{\prime 26}$ All of these definitions point to the problem of defining the borders of just what a musical instrument actually is and just what instrumentality might mean. Any definition must show how instruments are inseparable from their use, in addition to being a physical objects and a transformer of action.

\section{Artist Writings}

\footnotetext{
${ }^{224}$ Jordan Bartee, "Ontological Toys” (PhD dissertation, Brown University, 2014), 57.

${ }^{225}$ As quoted in Kvifte, "What Is a Musical Instrument?" 46.

${ }^{226}$ Brian Clarence Hulse, "Thinking Musical Difference: Music Theory as a Minor Science," in Sounding the Virtual: Gilles Deleuze and the Theory and Philosophy of Music, eds. Brian Clarence Hulse and Nick Nesbitt (Farnham, England, Burlington, VT: Ashgate, 2010), 44.
} 
"The instrument-that's the matter-the stuff-your subject."-Steve Lacy ${ }^{227}$

Considerations of musical instruments from within creative practices are present or implied in scattershot places, mostly within experimental music studies or in the writings of some composers and performers. A very direct consideration is John Corbett's Attalian parsing of the role of the instrument within improvisation. ${ }^{228}$ Such considerations come especially naturally to those interested in experimental music or to composer/performers or improvisers in discussion of their practice. Improviser Derek Bailey for instance describes musical instruments (in contrast to the classical tradition) as “...not just a tool, but an ally." 229 Writers about improvised practices are often similarly articulate: "For instance, the contingency and resistance of the material is not a matter of insufficient playing technique that could or should be completely mastered with practice and increased control. Rather, it plays a fundamental aesthetic role." ${ }^{230}$ Musical instruments are seen not as obstacles to be navigated or dominated, but instead are vehicles for collaboration. ${ }^{231}$ More implicit discussions of instruments pointing towards a creative utilizing of instrumentality can be found in the work of innovators like David Tudor, who used circuit design and spatial considerations as material. There are also numerous performer-side writings that deal with such issues within jazz and Western Art Music

\footnotetext{
${ }^{227}$ Quoted in Derek Bailey, Improvisation: Its Nature and Practice in Music (Ashborune, England: Da Capo Press, 1992), 99.

${ }^{228}$ Corbett, "Ephemera Underscored," 220.

${ }^{229}$ Bailey, Improvisation, 99.

${ }^{230}$ Haenisch, "Materiality and Agency in Improvisation," 167.

${ }^{231}$ Interestingly, this line of thought echoes Bruno Latour's summation of the non-human actor as a source of recalcitrance, as "as obstacles, scandals, as what suspends mastery, as what gets in the way of domination, as what interrupts the closure and the composition of the collective. To put it crudely, human and nonhuman actors appear first of all as troublemakers, with the notion of recalcitrance offers the most appropriate approach to defining their action." See Bruno Latour, Politics of Nature: How to Bring the Sciences Into Democracy (Cambridge, Mass: Harvard University Press, 2004), p 81.
} 
performance, though often obliquely and generally unsystematically. ${ }^{232}$ As noted earlier, there has also been a recent tendency to also consider action and gesture as material which points most directly towards the concept of instrumentality that I am suggesting. ${ }^{233}$

Composer Paul Craenen has attempted the most comprehensive account of such considerations in his book Composing Under the Skin: The Music-making Body at the Composer's Desk. ${ }^{234}$ While focusing mostly on how the body intersects with compositional process, sound and notation, he nevertheless spends quite a bit of time theoretically considering the instrumental interface as a bodily extension. ${ }^{235}$ While such work brings us one step closer to a functional concept of instrumentality within composition, it still somehow misses the mark, perhaps due to a focus on replicable, mediated forms of performativity. As a result, Craenen's concept of instrumentality is one-dimensional, and misses the opportunity of overlap in approach between not only avant-garde and experimental practices, but also more prevalent practices in music at large.

\section{Eidetic Variations and Phenomenological Music}

Composer/essayist Aaron Helgason comes a step closer to an explicit instrumentality. ${ }^{236}$

In his article "What Is Phenomenological Music and What Does It Have to Do With

Salvatore Sciarrino?" Helgason develops a theory and a methodology of

\footnotetext{
${ }^{232}$ Paul Berliner, Thinking in Jazz: The Infinite Art of Improvisation, Chicago Studies in Ethnomusicology (Chicago: University of Chicago Press, 1994).

${ }^{233}$ See Rolf Inge Godøy and Marc Leman, Musical Gestures: Sound, Movement, and Meaning (New York: Routledge, 2010) and Marc Leman, Embodied Music Cognition and Mediation Technology (Cambridge, Mass: MIT Press, 2008), 137-183.

${ }^{234}$ Craenen, Composing under the Skin.

${ }^{235}$ Craenen, Composing under the Skin, 106-168.

${ }^{236}$ The explicit integration of phenomenology into music has also appeared in the work of Don Idhe, Judith Lochhead, and Thomas Clifton.
} 
phenomenological listening ${ }^{237}$ He observes that phenomenologists, like Brentano, Hussurl, Heidegger, Merleau-Ponty, in general avoid music. ${ }^{238}$ Helgason defines and gives examples of phenomenological perception in relationship to music through intentional objects - objects of perceptual intent/attention/focus. ${ }^{239}$ Most important is that sounds are heard in relationship to the objects that make them. Helgason claims sounds in phenomenology complete our perception of objects in a category, pointing towards their eidetic variations. ${ }^{240}$ These eidetic variations result from a triangulation of what we know from our histories with the thing and its materials, and what the unavoidable haecceity of a particular moment reveals about it. This gives us as listeners a sense of their interior cartographies as a function of their phenomenological intersectionality gravity, wood, wire, and force. Though not explicitly about instrumentality, Helgason helpfully lays the groundwork for such an approach through a different type of connection to phenomenology derived more from Heidegger's mentor Hussurl.

\section{Affordances and Ecological Psychology}

Any full consideration of the musical instrument is inseparable from the actions that animate it and the sounds that result. Action, instrument, and sound as we observed earlier are intimately related, though not at all equivalent and always in flux. To Heidegger, action is implicit in how tools are understood in the "life-world" and how we develop our "tool-sight," which blinds us from the ontological reality of an instrument

\footnotetext{
${ }^{237}$ Aaron Helgeson, "What Is Phenomenological Music, and What Does It Have to Do With Salvatore Sciarrino?" Perspectives of New Music 51, no. 2 (Summer 2013): 4-36.

${ }^{238}$ Helgeson, "What Is Phenomenological Music," 5.

${ }^{239}$ Helgeson, "What Is Phenomenological Music," 6.

${ }^{240}$ Helgeson, "What Is Phenomenological Music," 7.
} 
even as we become a part of it. The same is true for Latour, whose concept of the actant is intimately related to understanding action. As a result, a connection between instrumentality and action is essential.

As we have seen, the emergence of instruments as objects is directly related to a new approach to sound and the parameterization of gesture, movement, and action. Juraj Kojs in his paper "The Language of Action and Cyberaction-based Music: Theory and Practice" attempts to define and indicate the origins of "action-based music," developing a theory of action-based music based on the concept of affordances. ${ }^{241}$ Drawn from ecological psychology, affordances were defined by James J. Gibson as what the environment or object affords the person or animal. ${ }^{242}$ Similarly to Heidegger, Gibson wanted the term to capture the complementarity of an animal and its environment, thereby diminishing the importance of judging an object only by its perceived qualities. ${ }^{243}$ Building on this concept, the psychologist W.W. Gaver states:

The concept of affordances points to a rather special configuration of properties. It implies that the physical attributes of the thing to be acted upon are compatible with those of the actor, that information about those attributes is available in a form compatible with a perceptual system, and (implicitly) that these attributes and the action they make possible are relevant to a culture and a perceiver. ${ }^{244}$

\footnotetext{
${ }^{241}$ Juraj Kojs, "The Language of Action and Cyberaction-Based Music: Theory and Practice," Journal of New Music Research 38, no. 3 (2009): 285-94.

${ }^{242}$ See James J Gibson, The Ecological Approach to Visual Perception (Hillsdale, N.J: Lawrence Erlbaum Associates, 1986), 130.
}

"The concept of affordance is derived from these concepts of valence, invitation, and demand but with a crucial difference. The affordance of something does not change as the need of the observer changes. The observer may or may not perceive or attend to the affordance, according to his needs, but the affordance, being invariant, is always there to be perceived. An affordance is not bestowed upon an object by a need of an observer and his act of perceiving it. The object offers what it does because it is what it is."

\footnotetext{
243 "The affordances of the environment are what it offers the animal, what it provides or furnishes, either for good or ill. I mean [by affordances] something that refers to both the environment and the animal in a way that no existing term does. It implies the complementarity of the animal and the environment." See Gibson, The Ecological Approach to Visual Perception, 119.

${ }^{244}$ William Gaver, "Technology Affordances," in Human Factors in Computing Systems Conference
} 
From this ecological vantage point, Kojs traces the history of types of music that are explicitly based on the exploration of affordances of an instrument, object, or situation through action. Though Kojs' interests lie more within the digital domain, such ideas are equally applicable to the acoustic domain as well, and moreover traditional musical instruments, from which most of his examples are drawn. For instance, in describing his concept of action-based music, Kojs states:

In music, interaction between a performer and instrument epitomizes such [ecological] actions. For example, a violin scratch tone - the exaggerated frictional sound of the bow scraping across the strings of a bowed string instrument-reveals not only the source of the sound production (a string instrument) but also an action type (scraping). Interaction between the performer's bow and the instrument's strings therefore not only fosters sound production, but also inspires our understanding of the sound production means and modes..$^{245}$

Kojs" definition of "action-based music" posits a type of music in which the awareness of the source of sound production, or an awareness of the perceptual and phenomenological aspects of sound, is essential. Kojs work which encompasses the materialist triad between the tools of sound production, the action type, both of which are indicated by, or result in, sound.

We see here two important things. First, there is the acknowledgment that actionbased ecological principles are not only present in such recent extended techniques but also within conventional violin playing. They are however typically obscured, by either habituation or context, similarly to how focusing on the content of a person's voice can deafen us to the sound of the voice itself. Kojs however is drawing our attention to kinds of music that force us to confront these properties, that make us experience anew - as

Proceedings (ACM Press, 1991), 79-81.

${ }^{245}$ Juraj Kojs, "The Language of Action and Cyberaction-Based Music," 286. 
audience member or performer alike - the phenomenal presence of instrument as sounding object, through action. Such ecological perception is not limited to scratch tones. Though action-based music often engenders an awareness of the source of sound production, such ecological perception is in fact available at any time, to any type of music or sound. Engaging in this type of listening can encourage an awareness of the perceptual and phenomenological aspects of sound, which encompasses a dialectal relationship between the tools of sound production and the action type, both of which are indicated by or result in sound. This typically obscured configuration of the materialist triad is what Kojs highlights in his analysis of music based on the ecological model.

Overall, Kojs' goal is a valuable one, establishing a place for action as an area of research and as a basis for compositional practices. However, action is also problematic to define. Unlike movement or motion - themselves complicated enough to understandaction also implies a layer of goal-oriented behavior. Action as a term is muddy, mired down in questions of intent, prone to point towards subjective phenomena.

In addition, when turning the word action back around to traditional music, is not all music action-based? Though Kojs' intent is to draw out the significance of actionbased practices, the actuality of music making is much more complicated, and not clearly divided up between actions and non-actions. Any human performer is involved with action in performance. Action in Kojs' context is more meant to show how action as material can lead us to new places and reveal new types of sound production. Kojs, in attempting to carve out a place for action within the field of composition, broaches a subject too applicable to all music isolate the music that he is clearly keen to investigate, like Kagel, Lachenmann, and others. Action provides a vital connection to 
phenomenology and the concept of affordances. On the other hand, such work gives us little information about how analyze and interpret the significance of instrumentality. As we have seen, instruments also have their own significant presence that remains largely unaccounted for. Though instruments are neither action nor sound, they play an essential role within the ecological model. Indeed, they are in fact the elements most directly analogous to how we imagine an actual ecosystem.

The work of Eric Clarke has similar advantages and drawbacks. In Clarke's analysis of Jimi Hendrix’s “Star Spangled Banner," he claims that perceptual meaning and value occur in a number of different domains simultaneously. ${ }^{246}$ He states: "Culture and ideology are just as material (in the concreteness of the practices that embody them) as are the instrument and human body that generate this performance, and, as perceptual sources, they are just as much a part of the total environment." ${ }^{247}$ Yet the story he tells is almost completely from the perspective of the listener's subjective experience. I do not argue with this point but, from the composition and analysis side, it does little to help us understand how the music is actually made and the significance of the electric guitar in within the compositional frame. This seems like a significant oversight, as the materialist perspective is an important component in building larger cultural theories of perception. For instance, Clarke talks a great deal about feedback from the listener's point of view, culturally and in regarding its nature as attack-less sound. ${ }^{248}$ But feedback itself forms a fascinating materialist ecosystem within a performative ecosystem, uniquely facilitated in this case by the electric guitar/amplifier/performer connection. I use this example not to

\footnotetext{
${ }^{246}$ Eric F. Clarke, Ways of Listening: An Ecological Approach to the Perception of Musical Meaning (New York: Oxford University Press, 2005), 61.

${ }^{247}$ Clarke, Ways of Listening, 61.

${ }^{248}$ Clarke, Ways of Listening, 61.
} 
criticize Clarke's work per se but to show how, overall, theories of ecological perception when tied to music tend to focus on esthesic properties, like the listener's perceptual or phenomenological experience, while largely ignoring both the immanent level of the musical structure and the materialist levels of the poietic side. So, while the expanded field of ecological perception, extends to encompass the physical, psychological, and cultural, the human and non-human elements alike, within the frame of music. However, the materialist case has yet to be made.

Though more recent work by Clarke attempts to rectify this to some degree by discussing instrument-specific rehearsal elements of new music, it still evades the materialist question by choosing to focus on the social aspects of rehearsal as opposed to the structural elements at play in actual performance. Enactive processes involved in performance and the affordances of the musical instruments themselves within that ecology are unaccounted for. From such a vantage point, every element of this obscured materialist configuration of action-instrument-sound is important. The instrument and its affordances, though recognized, remain oddly invisible.

Nevertheless, the basic picture presented here of music as an assemblage of human and non-human factors is a powerful one. ${ }^{249}$ It obliquely relates to DeLanda's assemblage theory derived from Deleuze and Guitarri. In Clarke's attempt to "materialize" non-material elements, he provides a model for how one could rethink an entire performative assemblage. However, this quote is also evidence of the problem with Clarke's work for this particular application. Its focus is clearly on listener perception. Clarke's work puts theories of ecological perception to use in attending to Nattiez's

${ }^{249}$ Assemblage is more strictly a Deleuzian term, not a Clarke's per se. 
esthesic properties - the listener's or audiences' perceptual, phenomenological, or interpretive experience, while largely ignoring both the immanent level of the musical structure and the materialist components as experienced from the performer's side (Nattiez's poietic).

There are other bodies of similarly situated scholarship within music that provide a basis for a concept of instrumentality through action, especially through a combination of technology, composition, and gesture studies. Composer and improviser Newton Armstrong achieves this synthesis vis-à-vis ecological phycology, the phenomenology of Maurice Merleau-Ponty, and Francisco Varela. His work is especially significant for importing the concept of enaction into music. There is also a large body of speculation as to the nature of instruments from within electronic music and those concerned with new musical interfaces. The navigation of the ontological weirdness of digital instrumentality has resulted in analyses of instrumental interaction geared towards understanding and promoting new technologies. ${ }^{250}$ However these theoretical and ontological investigations deal with digital technologies which have its own particular concerns so are therefore of limited use for development of a broader theory.

\section{Ontological Toys for Ecologonometric Play}

Jordan Bartee in his dissertation "Ontological Toys" makes a sustained case for the application of neo-Heideggerian, non-correlationist thought via object-oriented ontology to the confluence of music composition, instrument making, and computer programming. He broadly defines traditional musical instruments as he navigates the ontological

\footnotetext{
${ }^{250}$ The general concerns of conferences like NIME (New Interfaces for Musical Expression) are an example of such a community.
} 
complexities brought about by new technologies, especially those of the digital variety. Bartee's motivation is a deeper understanding of highly malleable electronic instruments like synthesizers (he calls them instrument-producing instruments) while still differentiating them in type from purely acoustic instruments. Bartee's solution is a concept of instrumentality that facilitates rethinking all musical instruments - acoustic, electronic, and digital — as systems that enact possibilities through work or play.

Bartee takes as axiomatic that all instruments are structural. Furthermore, he states that musical instruments should not be confused with their sounds, a somewhat startling claim; they are instead a "unique possibility matrix" in which instrument and player are fused into a single unit (similar to the composite body but without the "body of knowledge"). The actions of the performer engage with this matrix. He places an important value on play, stating: "[Musical] instruments are manmade systems that enact possibilities from limitations through work or play" and that play is "an important metric to consider when differentiating instruments." Yet this type of play, common to synthesizers or software for instance, is not by necessity foreign to acoustic instruments.

The term ecologonomy is taken from video game composer and theorist David Kanaga ${ }^{251}$ It is formulated in reference to what he calls game form, though it is equally applicable to my conception of instrumental practice as an assemblage of actants. Kanaga observes that the prefix eco- originates in the Greek word oixos, meaning household. It follows that the word "economy" means literally "management of the household" while the word ecology means "the ground of the household." ${ }^{252}$ Kanaga goes on to situate economy as a concern with rules. For Kanaga and video games, this means game theory.

\footnotetext{
${ }^{251}$ David Kanaga, "Wombflash Forest: Intro to Ludic Ecologonomy (Pt. 1)," Wombflash Forest, July 26, 2015, http://wombflashforest.blogspot.com/2015/04/intro-to-ludic-ecologonomy-pt-1.html.

${ }^{252}$ Kanaga, "Ludic Ecologonomy."
} 
In my transposition of Kanaga's model from game theory into instrumental practice, the implication is that elements of musical practice that are well established but ultimately circumventable: aesthetics, history, culture, training, tradition, performance culture, composition, notation-music-making as a set of explicit and implicit rules.

Essential to this concept is the idea that rules can be broken. Rules are conventions, but not elements inherent to design or physics. Affordances might point to musical rules that resulted in a certain instrumental design, but that does not mean other interactions are not possible. In instrumental practice this could be something as basic as "the bow is supposed to make contact with the strings with the hair most of the time." These types of rules provide the basis for how we interpret normative instrumental activity. Economy, on the other hand, means the ground of the household: the musical instrument as a system of affordances, in the senses defined by the ecological psychology of Bateson, Gibson, Gaver, and the writings of David Norman. These are the aspects of an instrument that are inherent to its objecthood, to its existence in the physical world, and to its intersection with the instrumentalist. The combination is conglomerated into a distinctive portmanteau, ecologonomy.

Ecologonomy implies a complex equilibrium. Kanaga uses the example of basketball, where normally you try to get the ball in the hoop as often as possible. But of course, you don't have to. And a basket does not have to be worth 1, 2, or 3 points. It could be anything. The game is the economic aspect. However, this does not change the essential nature of the basketball itself, or the hoop, or the court, or gravity. This is the ecological aspect. Combined, they form an ecologonometric space. A radical of the household, of course, can alter this equilibrium into non-existence. Musical instruments 
are material architectures that define interactive systems through play ${ }^{253}$ However, if this is true, different instruments, while ontologically united, have radically different and aesthetically relevant differences. A piano in performance points for instance to gravity in a way that laptops do not. Different instruments afford and facilitate different types of play. Ecologonometric play can be undertaken with any instrument or object. Furthermore, any element that comprises or augments an instrument can be explored. Ecologonometric play can also be undertaken with any element of the musical assemblage.

Terminologically, the idea of ground bridges the etymologically interrelated concepts terrain, terroir, and territory. By also borrowing from its Deleuzian heritage, we find a rich terminological intersection that can be folded into ecologonomy. A territory is of course not the map, which could be thought of as the musical notation. A territory is also not pictures or recordings of what can be found within it. But neither is the territory the land itself. It is all of these and none of these. Ecologonometric play with musical instruments or instrumentalized objects can be thought of in a similar way, with the instrument itself as the territory in this complex sense. Such types of play in performance can be thought of as kinds of play that, self-consciously or un-self-consciously, foreground instrumentality.

This brings us to an essential conclusion: that strategies for ecologonometric play are a form of composition. The instruments, the strategies for actions, the interactive, inter-objective field of play involved in performance-all can be included under the umbrella of "acts of composition." Through thinking musical instruments as "actants of

\footnotetext{
${ }^{253}$ Bartee, "Ontological Toys,” 49.
} 
composition" as in Chapter 1, it becomes clearer how both instruments themselves and strategies for ecologonometric play with them can become acts of composition.

\section{Instrumentalization}

"What human beings are and will become is decided in the shape of our tools no less than in the action of statesmen and political movements."

- Andrew Feenberg ${ }^{254}$

Another way to think about musical instruments is from the perspective of instrumentalization theory. Instrumentalization theory has been a central topic for philosopher Andrew Feenberg. Recalling Stiegler, Feenberg maintains that any technology must always be analyzed at two levels, that of the primary and secondary instrumentalizations, or the level of design and at the level of implementation. ${ }^{255}$

Instrumentalization theory holds that technology must be analyzed at two levels, the level of our original functional relation to reality and the level of design and implementation. At the first level, we seek and find affordances that can be mobilized in devices and systems by decontextualizing the objects of experience and reducing them to their useful properties. This involves a process of deworlding in which objects are torn out of their original contexts and exposed to analysis and manipulation while subjects are positioned for distanced control. ${ }^{256}$

Feenberg is concerned with transitions, transformations, and decontexualizations of technology. Unlike Heidegger, he not interested in tools in the ontological sense per se but rather in how our relationships to them change and how these can be expressions of power. His ideas about instrumentality are representative of the "old" materialism — that of Marx and Marcuse but combined with insights from Heidegger and others. He is

\footnotetext{
${ }^{254}$ Andrew Feenberg, Transforming Technology: A Critical Theory Revisited, 2nd ed (New York: Oxford University Press, 2002), 3 .

${ }^{255}$ Andrew Feenberg, "Critical Theory of Technology: An Overview" 1, no. 1 (Winter 2005), 47, 50.

${ }^{256}$ Feenberg, "Critical Theory of Technology," 50.
} 
largely concerned with power dynamics, politics, and industry. However there are useful insights, and not only because many musical instruments are industrially produced.

Of special interest with Feenberg is the idea of multiple levels of instrumentalization. Feenberg describes two levels of instrumentalization, primary and secondary. The primary level simplifies objects and things for integration - often as tools, equipment, or instruments-into both the natural and social environment. Though Feenberg is largely concerned with industrial processes, his insights still hold true in this case, especially when considering how instrumentality can shift as the actual object remains the same.

At the second level [of instrumentalization], we introduce designs that can be integrated with other already existing devices and systems and with various social constraints such as ethical and aesthetic principles. The primary level simplifies objects for incorporation into a device while the secondary level integrates the simplified objects to a natural and social environment. This involves a process which, following Heidegger, we can call "disclosure" or "revealing" of a world. Disclosing involves a complementary process of realization which qualifies the original functionalization by orienting it toward a new world involving those same objects and subjects. ${ }^{257}$

Important to Feenberg is retaining analytical distinction between two levels. These distinctions have the benefit of embracing both the "old" materialist of Marx and the "new" materialisms of science studies, ecological psychology, and Latour.

No matter how abstract the affordances identified at the primary level, they carry social content from the secondary level in the elementary contingencies of a particular approach to the materials. ${ }^{258}$

It is important to note the Feenberg thinks of primary and secondary instrumentalizations as dialectical. They almost never exist in isolation. ${ }^{259}$

\footnotetext{
${ }^{257}$ Feenberg, "Critical Theory of Technology," 50.

${ }^{258}$ Feenberg, "Critical Theory of Technology," 50.

259 "Similarly, secondary instrumentalizations such as design specifications presuppose the identification of the affordances to be assembled and concretized. This is an important point. Cutting down a tree to make
} 
In application to music, certain non-traditional types of actives can be thought of as can also be thought of as secondary instrumentalizations, though of a quite specific kind. A scratch tone on the violin, or playing inside of the piano, are types of secondary instrumentalizations. Modifying design to accommodate new values. But it can never escape where it came from.

The artistic activity remains technical and presupposes a primary instrumentalization, but the emphasis on aesthetic qualities of the artifact differentiates it from ordinary technical work in modern societies. This partial separation of the levels of instrumentalization encourages the false belief that they are completely distinct. This obscures the social nature of every technical act... ${ }^{260}$

Instrumentalization, and thus music, takes place in a complex social domain of interrelated primary and secondary instrumentalizations. Instrument makers, composers, performers are always already ontologically united in a technical assemblage. This becomes more complicated as non-instruments are instrumentalized, as secondary instrumentalizations proliferate. But secondary instrumentalizations can also take as their topic primary instrumentalizations as well, like wood, sound, or action. Such interpretations are obviously highly contingent on perspective.

\section{Instrumentalizing: From Noun to Verb}

A different strategy might be to consider instruments not as static objects, but as processes. Andy Keep in his article "Improvisation with Sounding Objects in Experimental Music" describes the process of instrumentalizing in terms of engaging

lumber and building a house with it are not the primary and secondary instrumentalizations respectively. Cutting down a tree "decontextualizes" it, but in line with various technical, legal and aesthetic considerations determining what kinds of trees can become lumber of what size and shape and are salable as such. The act of cutting down the tree is thus not simply "primary" but involves both levels as one would expect of an analytic distinction." Feenberg, "Critical Theory of Technology," 50.

${ }^{260}$ Feenberg, "Critical Theory of Technology," 50-51. 
with "the emerging sonic properties of an adapted or appropriated sounding object." To Keep, instruments can be acts. This process of instrumentalizing is aestheticsization of an object's affordances, expressed in sound. As a result, Keep states:

The performer's perspective of a musical instrument is also effectively changed from the traditional role of being a predetermined thing that realizes a musical language outside or indifferent to its self, to begin an act that explores an object for its inherent sonic properties. ${ }^{261}$

Keep is interested in improvised practice and invented/adapted instruments. He claims that creative instrumentalizing is an exploratory process seeks respond to the sonic properties of an adopted or appropriated sounding object, and in that way finds the "performabilty," the intrinsic sonic palette and possibilities for the sonic manipulation of objects." ${ }^{262}$

Examples of instrumentalization of objects are numerous and varied. Early examples we have already seen include Varese and Cage, especially pieces like the Imaginary Landscapes series and Water Walk; also Tudor's Rainforest series and Lucier's Opera for Objects. Other interesting examples, just to name one type of object, would be pieces utilize microphones as instruments. An entire book has been written on this single, seemly limited topic. ${ }^{263}$ Influential works that explore this modality of instrumentality include a cross-section of compositional practices: there is Stockhausen's Mikrophonie I (1964) from the European avant-garde and Steve Reich's Pendulum Music (1968) more aligned with the American experimentalist tradition; later works like Alvin Lucier's Bird and Person Dyning (1975) and the later Music for Piano with Amplified

\footnotetext{
${ }^{261}$ Andy Keep, "Instrumentalizing: Approaches to Improvising with Sounding Objects in Experimental Music," in The Ashgate Research Companion to Experimental Music, ed. James Sunders, Ashgate Research Companion (Farnham, England, Burlington, VT: Ashgate, 2009), 115.

${ }^{262}$ Keep, "Instrumentalizing," 115.

${ }^{263}$ Cathy van Eck, Between Air and Electricity: Microphones and Loudspeakers as Musical Instruments (New York: Bloomsbury Academic, an imprint of Bloomsbury Publishing Inc, 2017).
} 
Sonorous Vessels (1990). In all of these cases, microphones are explicitly instrumentalized, becoming part of the framework of the performance and composition. Instrumentalization theory when applied to musical instruments allows us in some measure to retain the insights of Heidegger while still accounting for the significance of change in the "body of knowledge," e.g. in the realm of the social. However even primary and secondary instrumentalizations are not adequate for the full hybridity, the push and pull of Latourian ontology. Even more difficult is accounting for the ways in which, as Stiegler says, artistic tools create a "futurity." Such processes are more clear when a "non-instrument" is instrumentalized. This requires decontexualization and a reterritorialization, which "shatters pre-exiting natural arrangements." ${ }^{264}$ Significantly, these arrangements can also be of a social or an aesthetic type. This process is different when the object is a traditional musical instrument: in such cases, the instrument is reinstrumentalized.

\footnotetext{
${ }^{264}$ Feenberg, "Critical Theory of Technology," 57.
} 


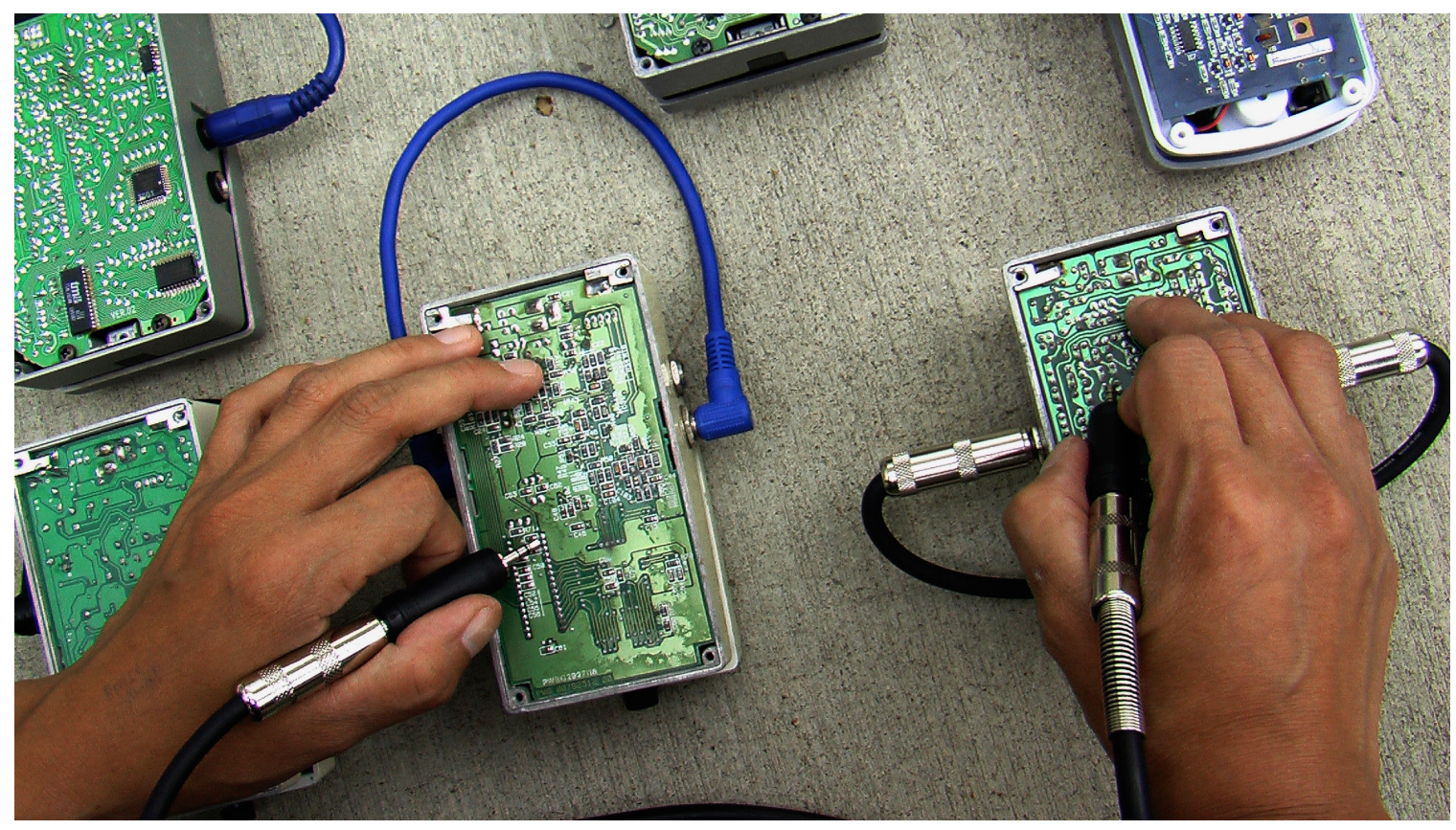

FIGURE 17: IMPROVISER BONNIE JONES' NETWORK OF INSTRUMENTALIZED DELAY PEDALS²65

\section{Reinstrumentalization}

I propose reinstrumentalization as a broad term to encompass all aspects of the composite body -individual relationships, cultural expectations, instrumental design, actions, performance, research, and more. It is a multidimensional process that might utilize any aspect of this assemblage as creative material. The relationship to the technical in the complex sense, the inherent sociality of the object, and the hidden affordances of the design all play important roles in reinstrumentalization. ${ }^{266}$

Reinstrumentalization presents challenges to composers, performers (if they are different), and audiences. Reinstrumentalization is at home in the domain of performance or recording. It can be captured as a real-time exploration in the performative medium.

\footnotetext{
${ }^{265}$ Image downloaded from https://bonnie-jones.com/.

${ }^{266}$ As Matthias Haenisch notes, "The materialization of praxis in an instrument in no way entails limiting the performer to strict norms or rules based on technical requirements, for example to an exclusively 'reductive' attitude to playing." See Haenisch, "Materiality and Agency in Improvisation," 164.
} 
These are practices that are re-presentational ${ }^{267}$ This however becomes more problematic when a performer or composer tries to communicate about or replicate such techniques. This leads us to a separate and more complex domain, that of representation in notation. The challenge in such a scenario is presenting the performer something they are accustomed enough to so they do not dismiss it out of hand, yet different enough that they are able to understand the new technique.

Reinstrumentalization does not mean that an instrument can be a tabula rasa. First of all, it always interacts with its own history and expectations, the body of knowledge. The instrumental body is also full of affordances, known and hidden, activated by the human body in action. Conventional instruments are actants or objects that contain the priorities and objectives of its maker or makers. They contain the compounded ideas and world-views of countless people whose ideas contributed to them. It is a nexus of ideas, a stable intersection of the human and the non-human - the materials, culture, and work flowing through a thing. These two interconnected elements - culture and compounded presence-are analogous to the economic and ecological dimensions that form the ecologonometric instrumental space. They are everpresent in instrumental music. The musical instrument has a special ontological significance, as the musical instrument is both meant to be aestheticized and is the vehicle of aesthetic ideas.

Ecologonometric play and reinstrumentalization can work in conjunction. Practices can be aesthetically as representing a different kind of awareness of the nonhuman world and tool-usage. Furthermore, the performative interaction of conventional

\footnotetext{
${ }^{267}$ I use this idiosyncratically, but specifically: representation to refers to notational practices while representation to refer to recording practices that strive for verisimilitude.
} 
instruments and instrumentalized objects in combination has the effect of transforming both. Within this greater sense of instrumentality, such practices point towards how the very action of utilizing a physical object for music is a phenomenological gesture. It is a proto-aesthetic act of deep significance. To instrumentalize is to experiment and hear, to discover, to find pleasure, to build sound. To reinstrumentalize is to discover hidden affordances, to discover anew, to discover an assemblage of actants within an assemblage of actants. Music engaged with reinstrumentalization can be deeply reflective of its materials as ground or process, inevitably pointing towards how such musical activities can be interpreted a phenomenological exploration, a type of philosophy without words, an existential exploration in action.

\section{Representations of Reterritorialization}

\section{Musica Negativa}

An example of reinstrumentalization and reterritorialization can be found in the music of Helmut Lachenmann. Lachenmann made his compositional breakthrough in the late 1960s with pieces such as temA (1968). Beginning in this period, his music very selfconsciously utilizes instrumentality as a compositional resource. His music shows another strategy towards such explorations and how they might be interpreted within the field of notated composition. After all this destruction, if traditional musical instruments are not abandoned, what is their role in this new world? What relevance might they have? Or how might they be approached or productively interrogated?

We can see an answer to this question as can be seen in his piece Guero. In this 
piece Lachenmann's focus on instrumentality comes less from a desire to liberate or to create a confrontation with the sedimented layers of theatricality of the live performance situation; rather, his motivation was a mixture of political, historical, and aesthetic concerns, issues that that to him were inextricably linked. It can be tempting to see his early output as pure experimentation with sounds and instrumental possibilities opened up by the then expanding dimensions of compositional parameterization engendered by serialist thinking. However to perceive the music only in this way is to miss its true radicalism, its political edge, and its curious quasi-Marxist goals and contradictions. It also would miss how Lachenmann combined these ideas to simultaneously reveal the means of production and to reflect the bourgeois world's musical tools back upon them, but in a distorted fashion. And it would also miss the powerful new cartographies that his notational innovations contribute to opening up new vistas for exploration and development. Lachenmann imagines materials very literally, as the musical instruments themselves.

Representative of this political and existential engagement is Lachenmann's politically charged language from his article "Composing in the Shadow of Darmstadt," where he sums up Darmstadt in the 1950s, the pivotal origin period of Object Musik:

Darmstadt in the 1950s: it meant rising up and breaking out, rejecting the inherited tonal, philharmonic-orientated concept of material, along with all of the technical and aesthetic implications that had been cultivated and worn out in bourgeois musical life up to then (and still today) - rejecting it in favour of conceptions that redefined the basic systematic categories for each work, taking as their point of departure unmediated perception and the possibilities of guiding its acoustic components. ${ }^{268}$

Lachenmann's individual solution to this problem seems to bring bear the possibilities brought about by serialism's interest in parametric structuring and its focus on sound

\footnotetext{
${ }^{268}$ Helmut Lachenmann, "Composing in the Shadow of Darmstadt," trans. Richard Toop, Contemporary Music Review 23, no. 3/4 (2004), 43.
} 
quanta via musique concrète and Elektronische Musik's reappraisal of sound as material.

He even termed his style in the late 1960s music concrète instrumentale (see

Introduction). The liberation of "noise" and of the significance indeterminate elements of both sounds and performance is also relevant. However, we also see another element: an underlying Marxist agenda, one in the service of quite literally exposing the musical "means of production"-acoustically, physically, and performatively. The end result is a musical world in which instruments are reduced, from which emerge:

...new sonic contexts, new paratraditions based on the exhaustive definition and exploitation of playing techniques evolved exclusively from the permutated recombination of possible actions involving any part of the instrument's anatomy, thus thrusting into the foreground the mode of generation - the shaping - of each sound as an inalienable component of its aesthetic import. ${ }^{269}$

The result is "nothing less than a Cartesian reassessment of Western music and art in general" through a relentless interrogation of our standardized concepts of beauty. ${ }^{270}$

The result is a quite different form of composition that consists of a literal and figurative remapping of the composite body. To Lachenmann, composing means inventing an imaginary instrument and then displaying it in a rarified and not easily repeatable context. ${ }^{271}$ The act of composing means inventing an instrument. ${ }^{272}$ This is related to his general approach to composition, which seeks to question what compositional material is:

Composing as resistance to the prevailing concept of material means: casting new light on this concept of material, illuminating it so as to reveal and create awareness

\footnotetext{
${ }^{269}$ Ferneyhough, "Shaping Sound," 162.

${ }^{270}$ David Albermann as quoted by Ross Feller, "Resistant Strains of Postmodernism," in Postmodern Music/Postmodern Thought, ed. Judith Lockhead and Joseph Auner, Studies in Contemporary Music and Culture (New York, London: Routledge, 2002), 252.

${ }^{271}$ Tanja Orning, "Pression - a Performance Study," Music Performance Research 5 (2012), 15.

272 As quoted in Max Paddison and Irène Deliège, Contemporary Music: Theoretical and Philosophical Perspectives (Burlington, VT: Ashgate, 2009), 12.
} 
of what is suppressed in it. ${ }^{273}$

Tanja Orning, in a performance study on interprets this approach as signifying that the body of knowledge is, at least symbolically eradicated, and the performer is liberated, leading us to the revelatory power of the sound source - "the very material qualities and physical energies of the sound." ${ }^{274}$ That source is of course action applied to the instrument itself; in her words: “...the qualities of the sound result from the material conflict between cello, bow, wood, and strings." In this way the instrumental practice becomes compositional material. ${ }^{275}$

Such a foregrounding of performance and instrumentality can be interpreted as a type of phenomenological discourse through music. In the words of Seth Brodsky, even while Guero masquerades as blank musical erotica:

... [it] becomes a kind of double act of tracing which awakens two great objects, only the first of which is the piano-monster, with its imposing monolith-machinery and monumental historical baggage. The other object becomes the pianist's consciousness itself, and through extension the consciousness of the listener as well...Guero becomes a kind of existentialist teaching tool, a kind of symbolic index finger (via the actual index fingers) extended at the door of unexploited cognitive and performative opportunities. ${ }^{276}$

Such a hybridized musical practice, with an explicit focus on instrumentality, is evidence of post-Cageian, post-Lockwoodian existential investigation on the part of the composer, performer, audience, and scholar alike.

\footnotetext{
${ }^{273}$ Lachenmann, "Composing in the Shadow of Darmstadt," 49.

${ }^{274}$ Orning, "Pression," 29.

${ }^{275}$ Orning, "Pression," 29.

${ }^{276}$ Seth Brodsky, http://www.allmusic.com/composition/guero-for-piano-mc0002431564
} 


\section{Guero}

für Klavier

Helmut Lachenmann (1969) revidiente Fassung 1988

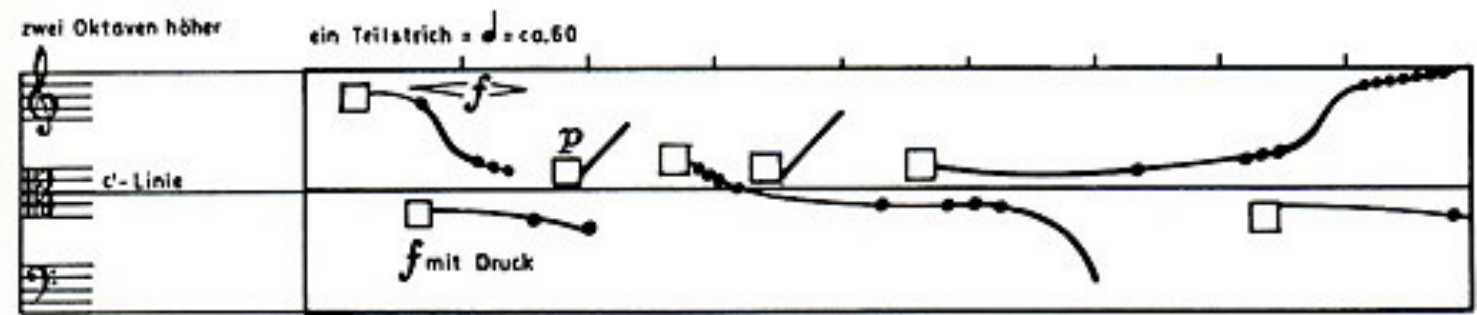

Iwai Oktoren tiater
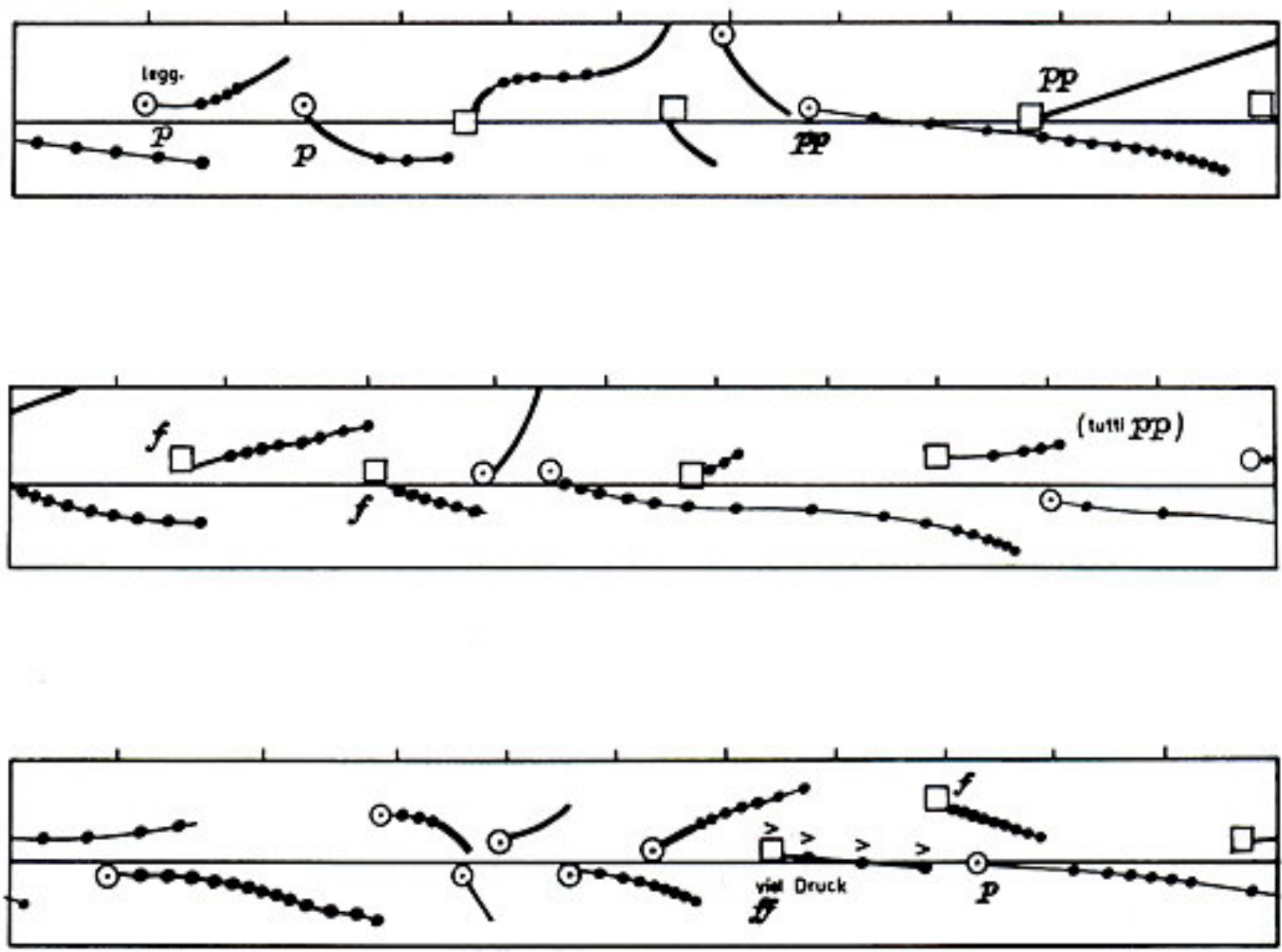

Edition Breitkopf 9018

6 1972 by Musikverlage Hans Gerig, Köln 1980 assigned to Breitkopf \& Härtel, Wiesbaden Printed in Germary 


\section{Wild "Things"}

Guitarist Jimi Hendrix, contemporaneously to Lachenmann in the late sixties, provides a different example of reinstrumentalization. Below are images of Jimi Hendrix at the Monterey pop festival in 1967. In this concert, Hendrix famously plays guitar behind his head, with his teeth, utilizes feedback, makes suggestive sexualized overtures towards the amplifier, and then sets the instrument on fire before smashing it on the ground seven times. These actions - which could be written off as merely theatrical, psychedelia-laced acts of one-upmanship or as shallow countercultural gestures-possess a deeper musical significance. Hendrix's guitar burning should not be construed a mere wonton act of destruction as in the case of other bands like the Who. As Pete Townsend himself stated: "for me, it was an act and for him, it was something else. It was an extension of what he was doing.".277

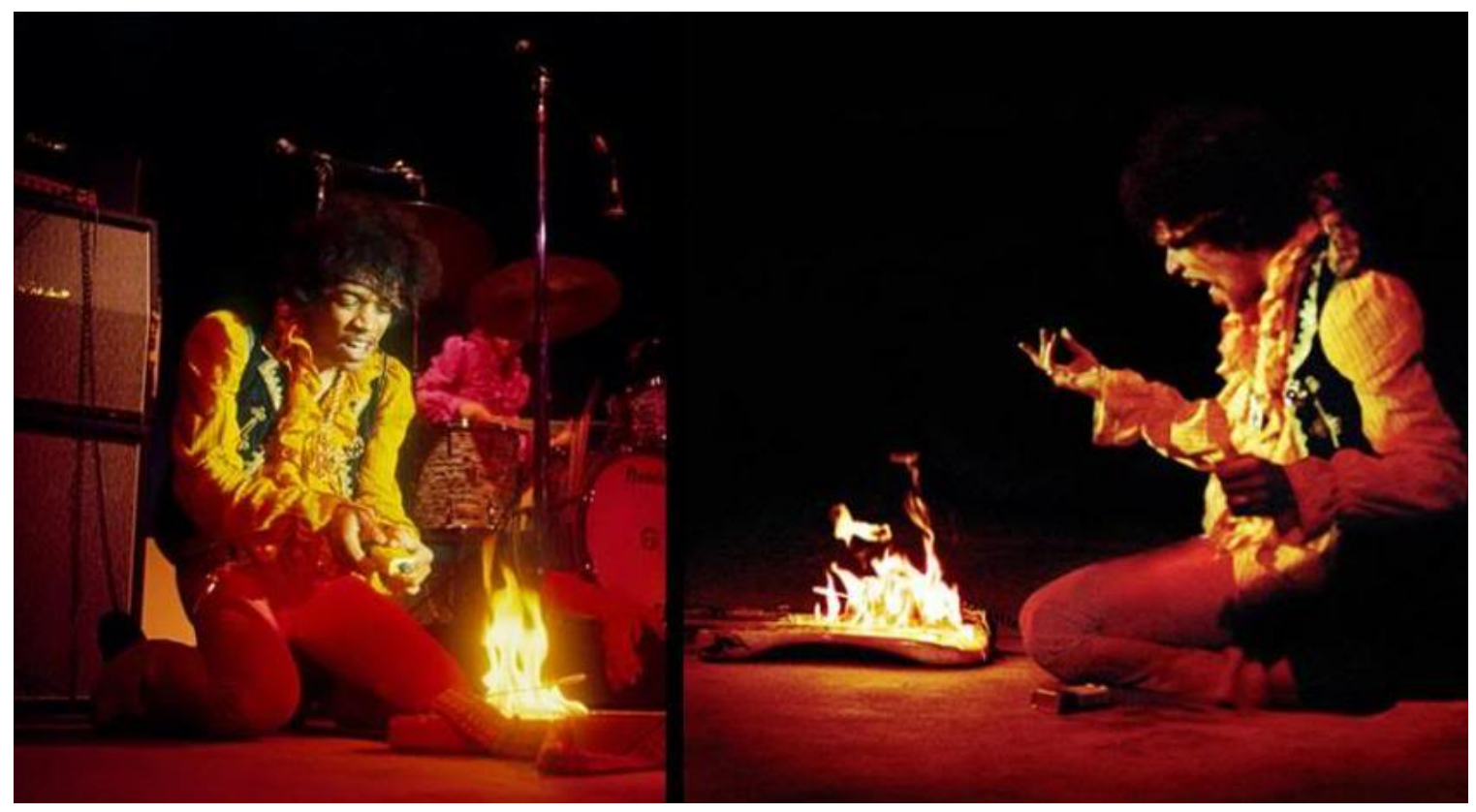

FigURe 18: Jimi HendRIX AT THE MONTEREy POP FESTIVAL

\footnotetext{
${ }^{277}$ Matt Wardlaw, "Pete Townshend Recalls Negotiating with Jimi Hendrix at the Monterey Pop Festival," Ultimate Classic Rock, October 26, 2011, http://ultimateclassicrock.com/pete-townshend-jimi-hendrixnegotiating-monterey/.
} 
Certainly these are techno-shamanic acts in which the guitar plays a role as totem and icon; but more than this, these actions happen in a context that is pushing the frame of not only the music but of the guitar itself outwards. The end result is at once a deterritorialization and a reterritorialization, a reinstrumentalization. Through the discovery of the novel possibilities of its augmented other, the electric "techno-phallus" that represents the fusion of man and machine that had suddenly usurped the traditional guitar; this assemblage of forces creates not only an extension of this hybrid instrument, but a complete decolonization of the traditional guitar, and in the process retranslating and transforming American blues, jazz, and popular music. ${ }^{278}$ Hendrix said: "When I burned my guitar it was a sacrifice. You destroy the things you love. I love my guitar.” It might have been a sacrifice, but it was also a sacrifice with a sound. ${ }^{279}$

What Hendrix is playing here is not just the guitar itself but the whammy bar, the amp, the fuzz pedal, the stage, even electricity itself. These instrumental extensions such as amplifiers and pedals in Hendrix's music are not merely theatrical window dressing. Technique and technology compress. The entire assemblage is utilized in a musical context. It is an assemblage of objects, people, actions, things, and forces, like electromagnetism. He is interacting in a contexture of distributed agency, a network in which the guitar is just one node. That this particular example is aided by technology is only an extension of the core context. The electricity that flows through the guitar literally completes the circuit of devices and amplifiers. They are instrumentalized into the assemblage of music making.

It is this assemblage and the contingencies of live performance that Hendrix must

\footnotetext{
${ }^{278}$ Waxman, "Instruments of Desire," 188.

${ }^{279}$ Bryan Wawzenek, "The Day Jimi Hendrix Set His Guitar on Fire for the First Time," Ultimate Classic Rock, March 31, 2015, http://ultimateclassicrock.com/jimi-hendrix-guitar-fire/.
} 
performatively and compositionally navigate. More than anyone else of his era in popular music, Hendrix musicalizes extended techniques. He deploys them at the right time in the set, in the right context in a song, and creates new contexts for them. He is also playing with the history of the instrument and the body of knowledge that accompanies it. It is of course true that certain techniques like playing with your teeth have not been popularized as mainstream technique, nor has the sound of guitar burning become a significant aesthetic practice. Certainly not every possible thing one can do with an instrument, just because it exists, is interesting. The interest is no exclusively in doing something new for its own sake, but creating musical meaning with such materials. As in Lockwood, this act is an end and a beginning.

\section{Musical Instrumentality}

Theories of objects, tools, equipment, and instruments, in the aggregate help to form a picture of a specifically musical instrumentality and the role it can play in the expanded field of contemporary composition. The concepts of actants, Unzuhandenheit, affordances, ecologonometric play, and reinstrumentalization all are helpful for understanding the nature of musical instrumentality. In the broadest sense, a concept of musical instrumentality must combine of all of these ideas and more.

In conceptualizing instrumentality and investigating the origins of music which

foregrounds it, I hope that the utility of the concept as both an analytical and creative tool is evident. There are many possibilities for approaching both analysis and composition from this perspective. Musical instrumentality can also enrich and re-contextualize the two other two elements of the materialist triad-action and sound - which both have 
ontologies and histories of their own that are interesting to pursue in a neo-materialist context. Instrumentality is just one piece of a materialist puzzle within musical assemblages. In exploring instrumentality I hope to point towards a hermeneutic that can facilitate understanding of both experimental and conventional musical practices, suggesting creative ways that such practices might be utilized in musical creation. Such a line of thought also points promisingly to ontologies of performance and of music composition.

The musical instrument is the complex actant, piece of nature-culture, a hybrid object. However instrumentality it is not only about the non-human or the physical, external object. As we have seen, this does not account for the singing voice or software instruments. To instrumentalize is ultimately an aesthetic act-it seeks to find or utilize the properties of a thing for music. In that way, instrumentality defines almost all musical practices in some way. Instruments, positioned as actants, form a significant a part of the fabric of any acts of composition of which music is composed.

The focus here has been squarely on the innovations of the experimental and avant-garde, especially the postwar origins of objectism in music in the long sixties, in order to contribute to certain reading and possible revaluation of experiential practices within the context of contemporary composition. However, in a broader sense, as I worked on this project I realized that, as aside from offering possible theoretical welcome to and an framing of non-score based/performative practices as compositional it became obvious that some form of instrumentality is a facet of all music. All music can be read through the lens of instrumentality. Instrumentality is a precondition for any sounding music. 
Though instrumentality is always present in music, the significance of this instrumentality is different in different musics. It is not equivalent, nor always equal in ratio to other factors. However, as we have seen, in many experimental and avant-garde practices instrumentality can also be a topic in and of itself - as the frame, the precondition, the mediator, the partner, revealing the terroir of the instrumental ground. To compositionally utilize instrumentality in live performance offers a form of haeccetic development inherent in the performative moment. But instrumentality is integral to any music, including the acousmatic. For to instrumentalize is at heart a transformative gesture. It is a distancing, a commitment to a special form of translation that straddles the borders between culture and nature. In this way, the use of instruments or the perception of instrumentality can be thought of as in essence a phenomenological gesture. Musical instrumentality can be interpreted as pointing towards the shadows of the human in the object through its design, affordances, and history, while also looking outwards towards the world through the materials and forces of which it consists via the actions that activate it, of which music is evidence. 


\section{Cartographies of Tool-Being}

\section{Reinstrumentalization as Composition}

An interest in non-traditional approaches to traditional instruments is reflected in my career both as an improviser and composer. Pursuing a conceptual expansion of what an instrument is and can do is an essential part of contemporary improvisation, and as we have seen, of certain approaches to contemporary composition. In my own work, a focus on reinstrumentalization is important. By specifically focusing on strategies of reinstrumentalization, these works are situated within the larger contexts of improvisation, experimentalism, and the avant-garde of Objekt Musik, and the broader world of musical objectism and sound art-all overlapping areas in the expanded compositional field. These pieces share a vision of composing through both subversion of and dialogue with the structurally coded nature of instruments and instrumentality. This is combined with an interest in sound and action in a search for ways to foreground the physicality and objecthood, the "grain" of the instrumentality in performance, and point towards the idea of a phenomenological music.

A fundamental mode of my practice as an improviser consists of utilizing the 
instrumentality of the cello as a medium. This often involves a breaking down of the cello's instrumentality — developing strategies for making the familiar foreign, in order to find new possibilities in elements that might be ignored or forgotten; or, put another way, a cultivation of cellistic Zuhandenheit. This approach evolved naturally through my involvement with free improvisation. In these communities, I often found the creative, idiosyncratic use of neglected or hidden instrumental affordances to be an implicit musical topic. Techniques based on an in-depth exploration of such affordances were then contextualized in performances and recordings. How improvisers research and develop these materials and techniques becomes an important component of artistry and the development of one's voice. The challenge later becomes how they are re-integrated with more conventional techniques or in collective settings.

Such an approach is not unique to free improvisation of course - many experimental musics foreground the real-time investigation of new techniques and the utilization of real-time structuring processes, be they physical, psychological, structural or acoustic, analog, or digital. These musics participate in an overlapping compositional dialogue in which instrumentality is important. However, such highly contingent materials often suggest their own native structuring possibilities, which can be difficult to access or develop in other ways besides individual performance. In the acoustic domain, the repercussions of this are generally intensive work within an individual performative practice. Compositional research in this context then often consists of not only of finding ways to make elements of the instrument foreign or find new ways of playing but to also develop a framework for "acts of composition" that organically compliment certain such highly embodied, performative approaches. I view work in this context as well within the 
frame of compositional activity, both as compositional research and as an element of the music's composition itself. These processes or remapping and reinstrumentalization are reflected in the accompanying work for cello, 14 Stations.

\section{Stations}

14 Stations for solo cello (2017) ${ }^{280}$ came out of a desire to develop a cello vocabulary of extremely limited means - through the playing the cello with a bow mediated by another bow. An example of this can be seen in the image below. This piece came as an outgrowth of an earlier piece, Music for 3, 2, 1, and 0 Bows for solo cello. In this piece, I experimented with a progression of kinespheres, or stations of physical and equipmental arrangements. These both gave structure to the piece despite elements of indeterminacy, through a circumscribing of movement areas or stations, defined by the physical arrangement of cello "equipment." 281 The challenge then as a performer became convincingly navigating the change from one movement area to another.

At one point in the piece I played one bow (in my left hand with the wood in contact with the strings) with another in my right hand. I found this to a fascinatingly limited repertoire of sounds to work with. This piece develops techniques of this one specific type of mediated playing, bow through bow in contact with different areas of the instrumental body. All 14 sections of the recording that accompany this document represent a specific physical arrangement (such as those above) aligned with a specific movement type. All of these sounds activate different areas of resonance of the

\footnotetext{
${ }^{280}$ See Appendix 1
} 
instrument, finding the hidden potential of pitch and noise achieved through the mediating through the bows themselves.

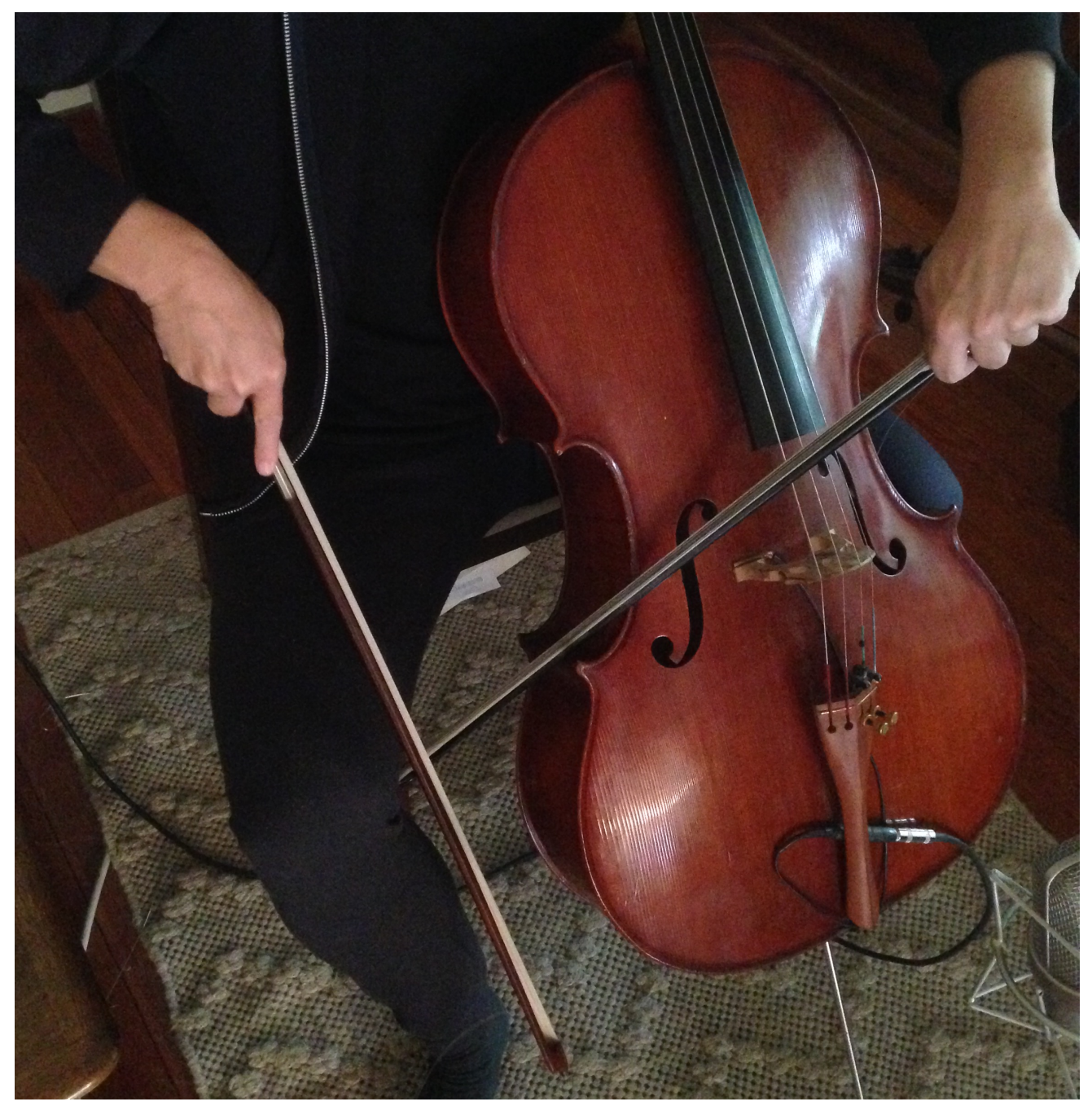

FIGURE 19: A PICTURE OF ONE OF THE "STATIONS"

As I was working, researching, and practicing of the different stations of movement involved, I began to notice an amusing resemblance to the Christian cross. This connection became a rich source of inspiration. This led quickly to engaging with Barnett Newman's "Stations of the Cross" paintings. These paintings used to confound 


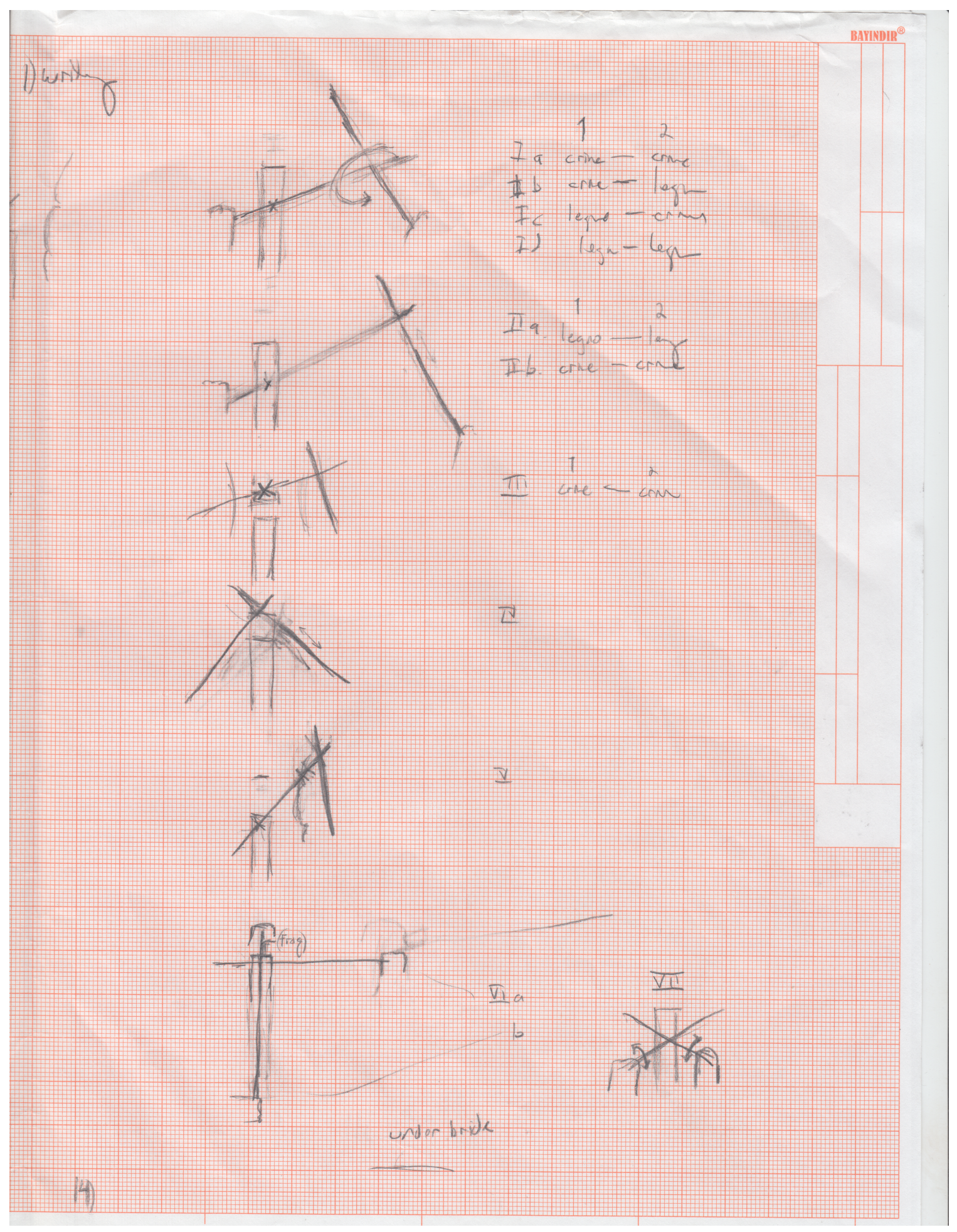

Figure 20: Sketches of "Stations" 
me with in their sparseness, their rigid formalism, and withholding of expression. They

have however grown on me over the years. In a sense, these pieces are an attempt to work through personal aesthetic issues related to similarly a restricted vocabulary with bracketed moments of expression and spontaneity.

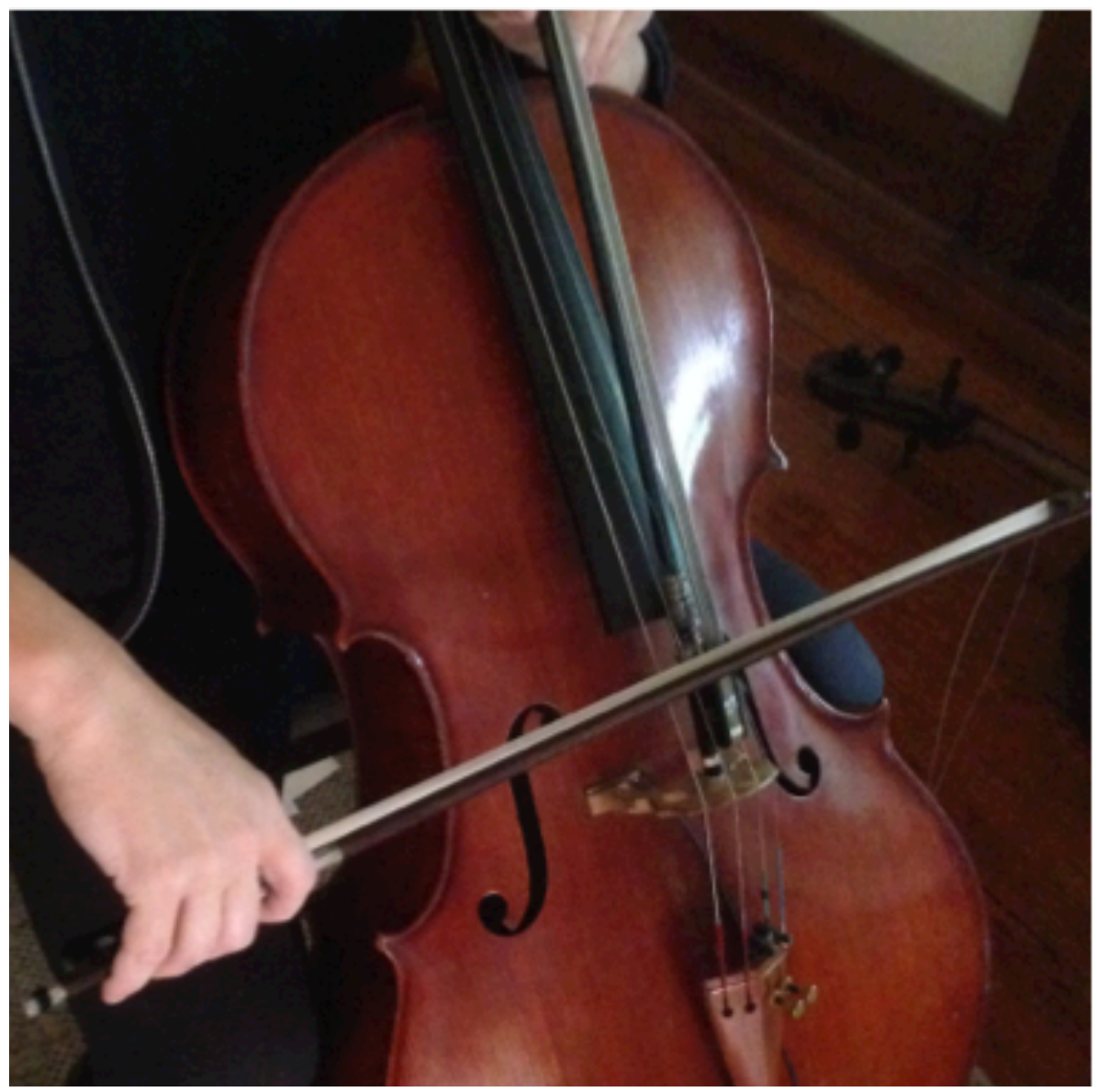

FigURE 21: PiCTURE OF ANOTHER "STATION." NOTICE OF THE LOWER PORTION OF THE LEFT HAND BOW IS IN CONTACT WITH THE BRIDGE AND FINGERBOARD AND IS BEING SECURED BY THE LEFT HAND, WHILE THE RIGHT HAND BOW IS IN CONTACT WITH THE HAIR JUST ABOVE THE FROG.

This work might be conventionally thought of as improvisation. It exists only a recording, the result of multiple iterations of performative research. However, I would 
also like to claim it as not only compositional research but as composition itself. Aside from the other more explicit compositional steps involved in the editing, selection, arrangement, and mixing, the sounds are structured by a history of reinstrumentalization.

\section{Notated Compositions ${ }^{282}$}

The other work included here grew out of attempting to break down divisions between improvisation and composition in my own creative practice. The challenge for me as an improviser is how to grow and move the music forward. The different but parallel challenge as a composer is to take this point-of-view and integrate it into conventional musical environments and/or standard musical notation. They are reflective of an interest in instrumentality the materialist triad overall, but also the evocative ideas of mistranslation and "anti-composition." The work here explores a spectrum of composedness and a variety of modalities. Compositions that foreground instrumentality, contingency, and the phenomenal moment of sound making have provided more commonality between these practices. Paradoxically, composing music that focuses on what I find interesting about improvisation often does not involve asking musicians to improvise. The pieces included here-Five Enactive Studies, Charm Against Loneliness, and 3 PFR-3 Poems by Jackson Mac Low-encourage engagements with performance, gesture, and physicality while not explicitly requiring improvisation.

\footnotetext{
${ }^{282}$ For recordings and further documentation, see http://kevindavismusic.com/works/
} 


\section{Five Enactive Studies}

For example, the Five Enactive Studies for piano (2010; revised 2013) ${ }^{283}$ represent a distillation of what I call the enactive approach. Movement, gesture, and physical process are utilized to produce developmental cohesion and organic variation, exploring the "grain" of the instrumentalist and of instrumentality. To foreground these phenomena, the pieces are constructed out of minimal sonic elements - trills, chromatic clusters, repetitive motions - all in states of perpetual flux. These materials are subsumed within the gradual arcs of other, larger intersecting processes, sometimes in response to coordinating subjective instructions (for instance "until the point of fatigue" or "as slowly as possible"). Subsequently, performer choice, the performer's ability, the performer's body, and performance space-in conjunction with the instrumentality and variations in the individual piano-are all significant.

\section{Charm Against Loneliness}

The next step in this approach is the piece Charm Against Loneliness for Chamber Ensemble (2016) ${ }^{284}$ Developed in collaboration with poet Courtney Fleberge and the University of Virginia New Music Ensemble, the piece embraces an anti-compositional ethos of various text transformations. This came about largely grew out of trying to solve notational problems involved with extended wind technique. Unlike the case of string instruments or piano, action-based notation is usually ineffective for flute or clarinet, as most of the action is coming from inside of the body, like the lungs, larynx, and mouth. ${ }^{285}$

\footnotetext{
${ }^{283}$ See Appendix 1

${ }^{284}$ See Appendix 2

${ }^{285}$ Thanks to clarinetist Shawn Earle for this insight.
} 
I came to the realization that we already have for gesture and movement notation that can easily be divorced from sound production while retaining its gestural qualities. In addition, we already have an in incredibly complex system to indicate what is happening in the mouth and throat: written language.

From this point of departure, I deduced to use conventional notation as much as possible while withholding different aspects of both tone production and by deconstructing the instrument itself-different types of Unzuhandenheit. Working from the letter of the poem as structural material, I developed various mappings of the alphabet to fingering patterns and gestural actions on the various instruments - playing without mouthpieces, reeds, or drumsticks. Normally well-defined sounds transformed into something more like gestures that produce, quiet, speech-like fragments. There is a literal and figurative breakage and reconstruction of both text and of instrumental technique. Especially interesting was the mapping of words and letters to the drum surfaces. This very simple transformation was capable of producing remarkable textural complexity.

\section{Embracing Mistranslation: Three PFR-3 Poems by Jackson Mac Low}

One of the interesting aspects of recent writings in $\mathrm{SR} / \mathrm{OOO} / \mathrm{NM}$ is the emphasis on mistranslation and misunderstanding. Heidegger presents a model of a world where the

thing is there but whose being is always furtive. Latour talks quite a bit about how objects translate each other in complex ways through networks. Harman combines and extends these two perspectives into a seeming inversion of reality - it is that which we cannot see that is real; all else is merely sense data and mistranslation, echoes of the structure of a thing. Indeed the written text itself can be thought of as a mistranslation of speech and 
perhaps even thought itself.

Following up on ideas from Charm Against Loneliness, the central compositional topic of the Three PFR-3 Poems by Jackson Mac Low for percussion quartet and speaker $(2017)^{286}$ is the transformation of poetry into sound through an embrace of mistranslation. The pieces experiment with multiple ways of achieving this, with instrumentality playing a crucial role in each. These three pieces are based some of the earliest computer generated poetry. These poems were composed 1969 at Information International, Inc., with the aid of a programmable film reader connected to a DEC PDP-9 computer and peripherals. There are a number of algorithmic processes involved in their composition; the actual text supplied by Mac Low consists of single or double lines or "messages" of less than 48 characters.

Since Mac Low both studied with John Cage and was known to derive poetry from music, it seemed an interesting project to return the favor. Of the three poems I selected, I used a variety of mappings based mostly on letters, but also stanza position and indentation. The poems are very straightforwardly mapped onto some musical parameters - like pitch, duration, and dynamics - and less straightforwardly mapped onto others, such as gesture or movement. The result is a simulacrum of not only language, but an odd sort of misrepresentation of the experience of reading an odd sort of poem.

This piece was developed in collaboration with the University of Virginia percussion ensemble. This gave me an opportunity to experiment with a number of different approaches. For instance, "From 'From David"” is for two standard drum sets and two percussion sets. In "From 'From David"” the musicians are given two 8-line

\footnotetext{
${ }^{286}$ See Appendix 3.
} 
staves, two for percussionists and two for standard drum sets. The choice of mappings for the drum set players is open; the two percussionists can map the notation to any objects they like in any mapping they like, as long as they can get two different sounds out of them. The mappings from word to letter to instrument become gesturalized, creating a cacophonous "reading" of the poem. From "From 'South"” is more compositionally straightforward. It consists of pitch mappings from the highest and lowest notes of piano a vibraphone respectively. The pitch mappings create their own peculiar harmonic and rhythmic language. The interest is more on the complexity of the emergent harmonic language and the accruing layers of sound. Also, there is also the additional challenge of how the mallet players divide up the excess of pitches. From "From 'The"” is the most complicated - and most collaborative - of the set. It is also the piece most explicit in its exploration of instrumentality. It came from the simple idea of mapping words into circular movements on a surface, a la Charm. I gave the percussionists the gesturalized versions of words:

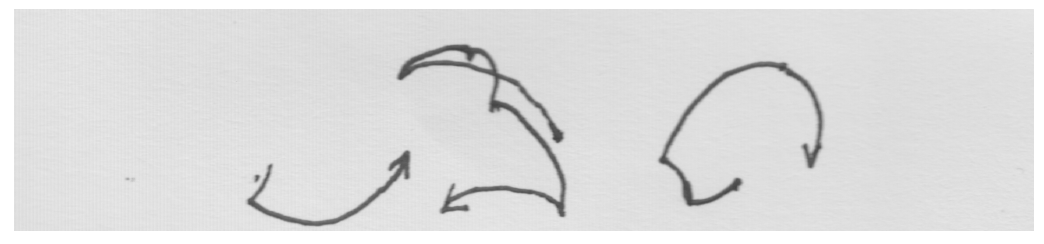

FIGURE 22: AN EARLY GESTURAL REPRESENTATION OF THE SENTENCE “THE PLANETS SHINE”

But now instead of using drums and cymbals as in Charm, the surfaces are different theaters of movement or kinespheres of the piano. This approach was inspired by pieces like Lachenmann's Guero (of which there is a pseudo-quote at the beginning) and the choreographic notation of Gordon Mumma’s Medium Size Mograph (see Chapter 2). The 
percussionists were given instructions to research the piano and find different areas and implements to perform these shapes and gestures that were interesting to them.

Subsequently, we reconvened and worked out the script and necessary choreography. This is now reflected in the score.

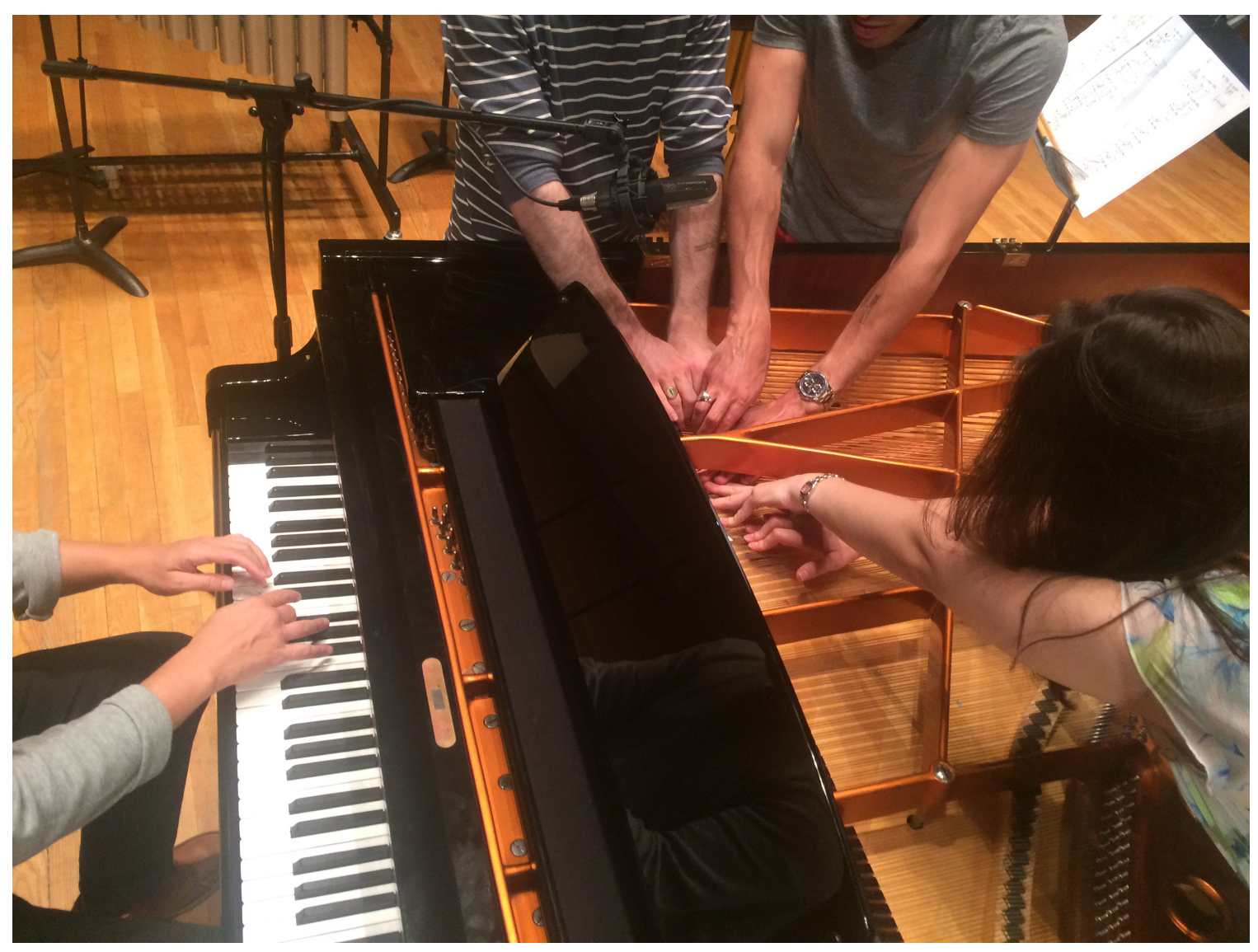

FigURE 23: EXAMPLE OF STOPPED HARMONICS IN STANZA 5 FROM FROM “FROM 'THE””

\section{Conclusion}

Ultimately, what is most is most musically interesting is not either Unzuhandenheit or Zuhandenheit in isolation, but in how these two modalities interact and how this tension can produce music of interest. Another way to see this is that I am interested in music that foregrounds the interplay of elements of John Corbett's triple body. Especially important 
is how the body of knowledge reflects both the instrumentalist's individual knowledge of an instrument and the cultural body of knowledge regarding that instrument. The area of overlap between these two is not exactly clear, which is what makes it such a rich area for artistic work. There is of course a tension between what is "composed" and the "improvised" in these works. And though I do not subscribe to the point of view that they are the same thing, I have found that this tension is a productive one, challenging and enriching for both. 


\section{Appendices}

Appendix 1:Five Enactive Studies for piano 


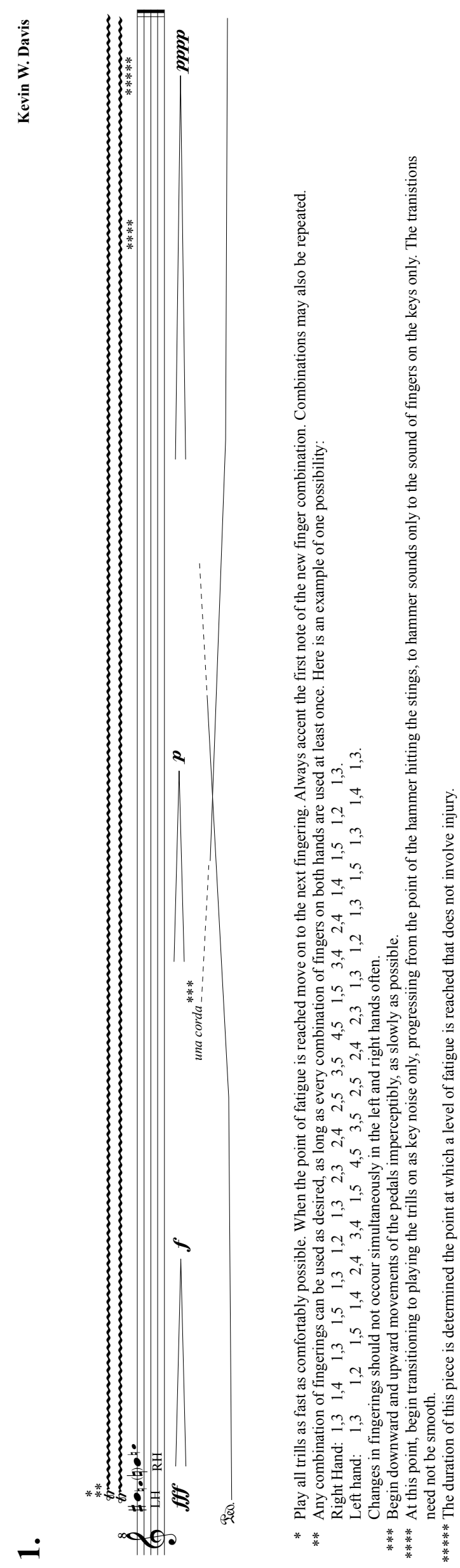



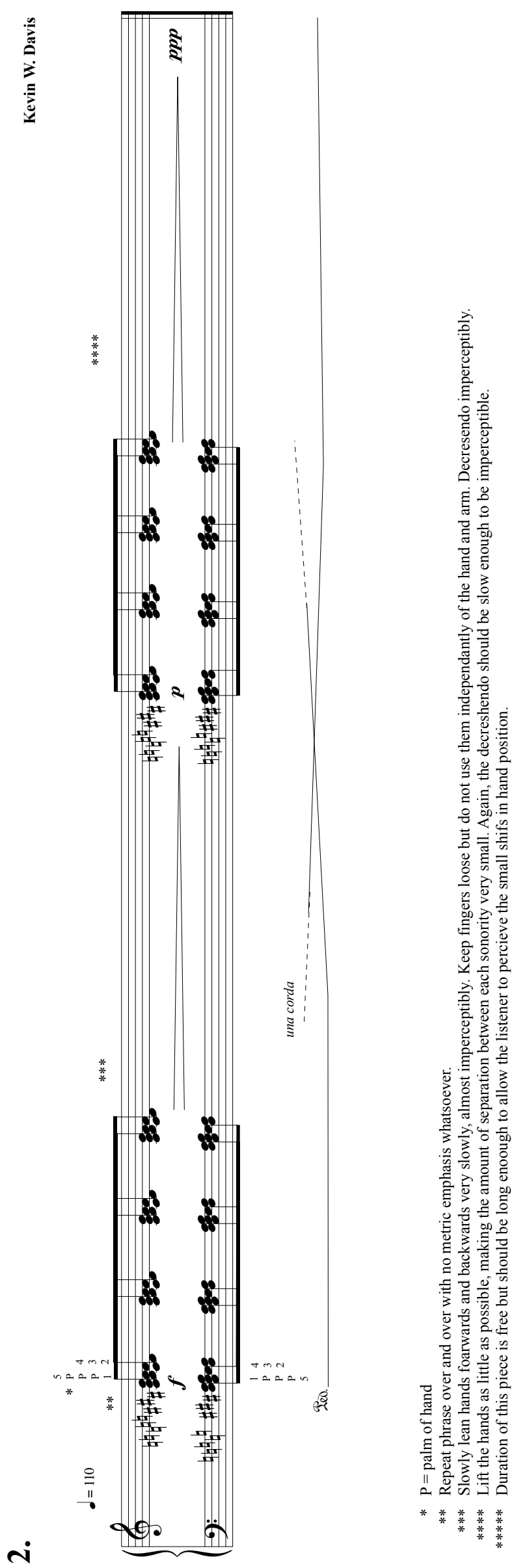


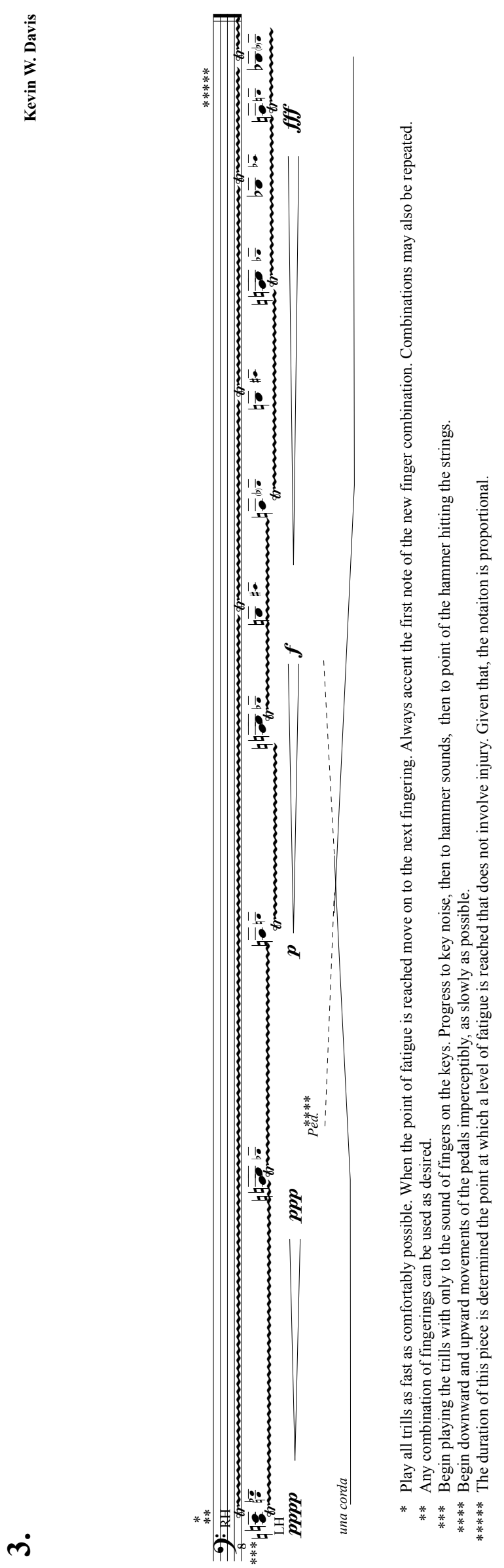




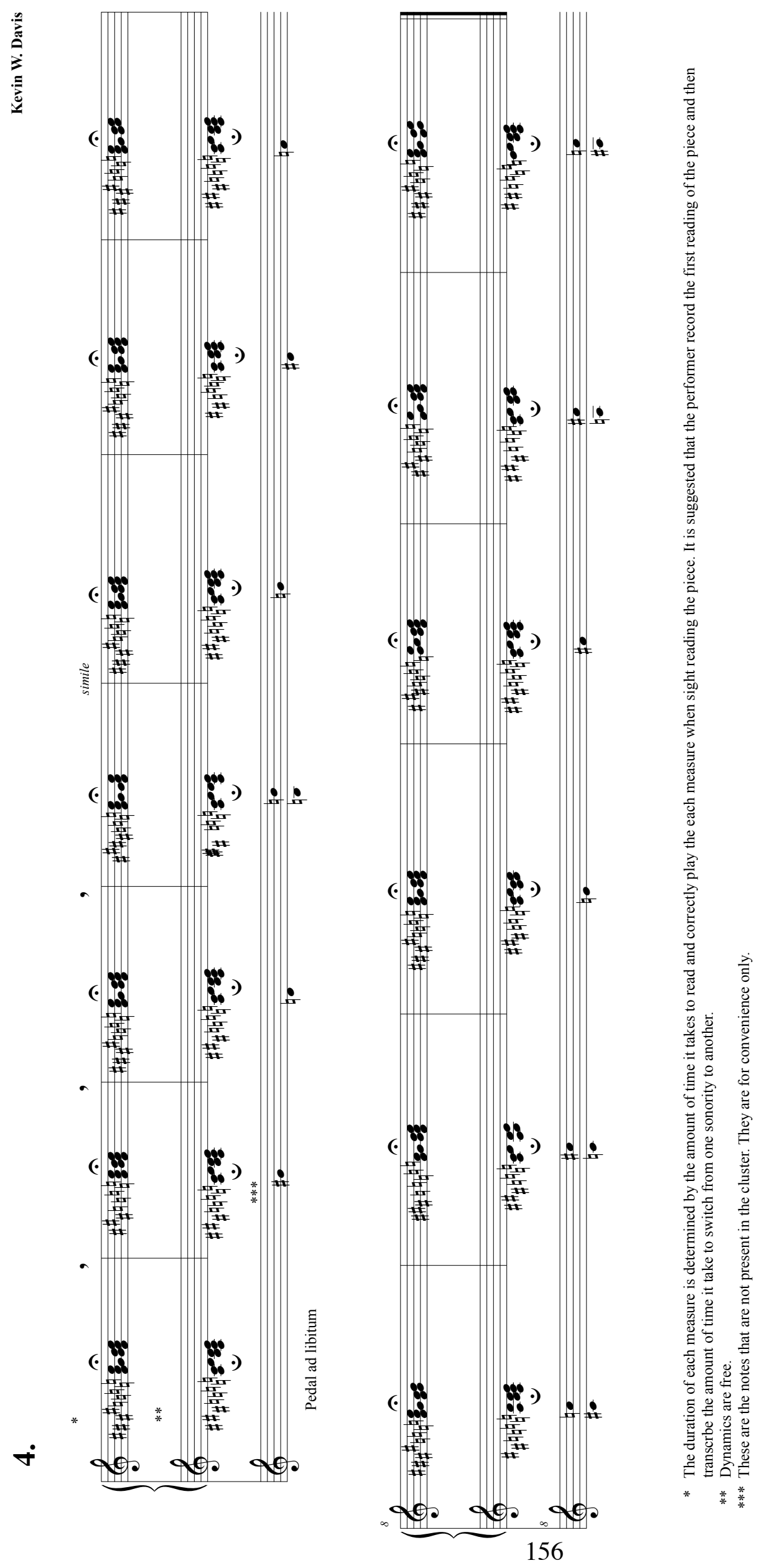




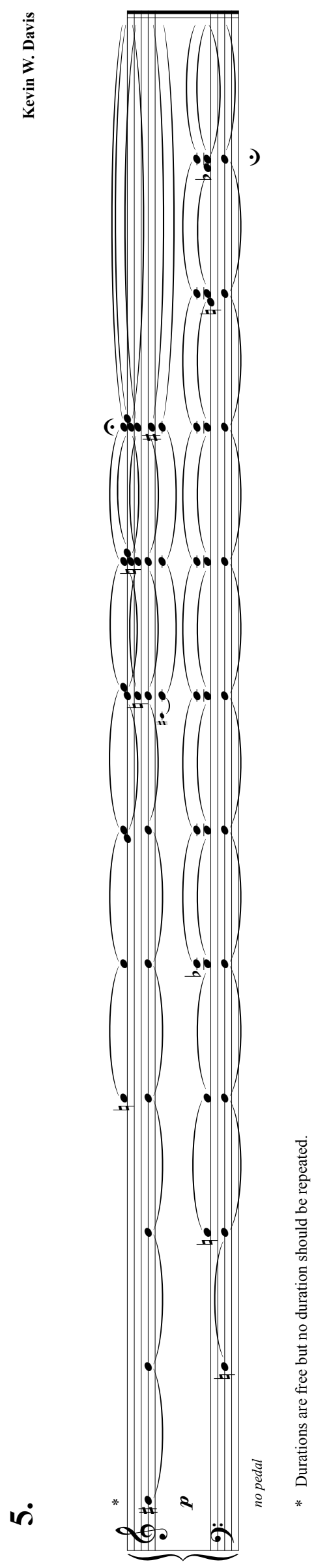


Appendix 2: Charm Against Loneliness 
Score

\section{Charm Against Loneliness}

from a poem by Courtney Fleberge

Kevin William Davis

Clarinet in $\mathrm{B} b 1$

1 Fast, speech-like

Flute

$0^{*}>$

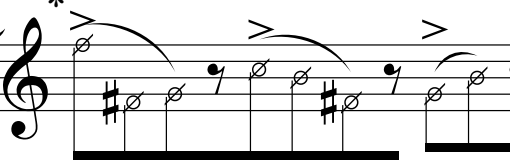

p

Clarinet in $\mathrm{B} b 2$

$\frac{0}{9}$

Q

Bassoon

9:

Tuba

\%:

Percussion 1 ıspended cymbal)

Percussion 2

(snare drum)

Percussion 3

(floor tom)

II

II
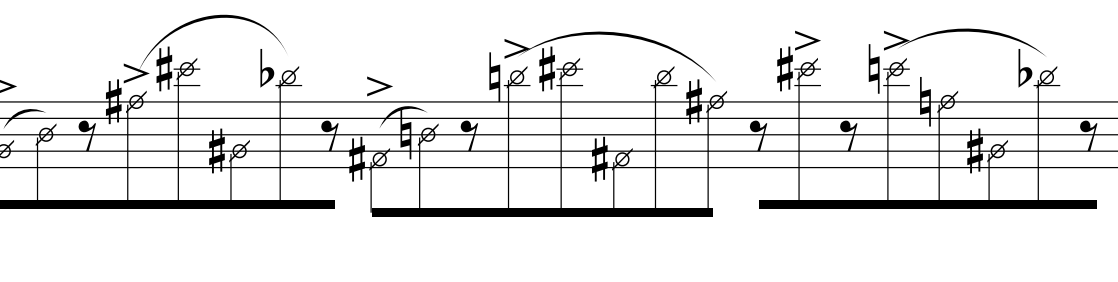
Fl.

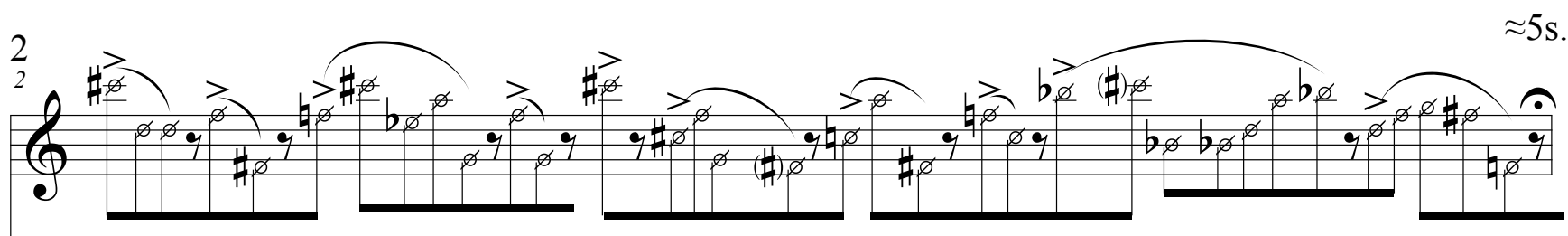

Vox

all it takes is a mist net

in dappled light,

Fl.

$\mathrm{B} b \mathrm{Cl} .1$

2

1.

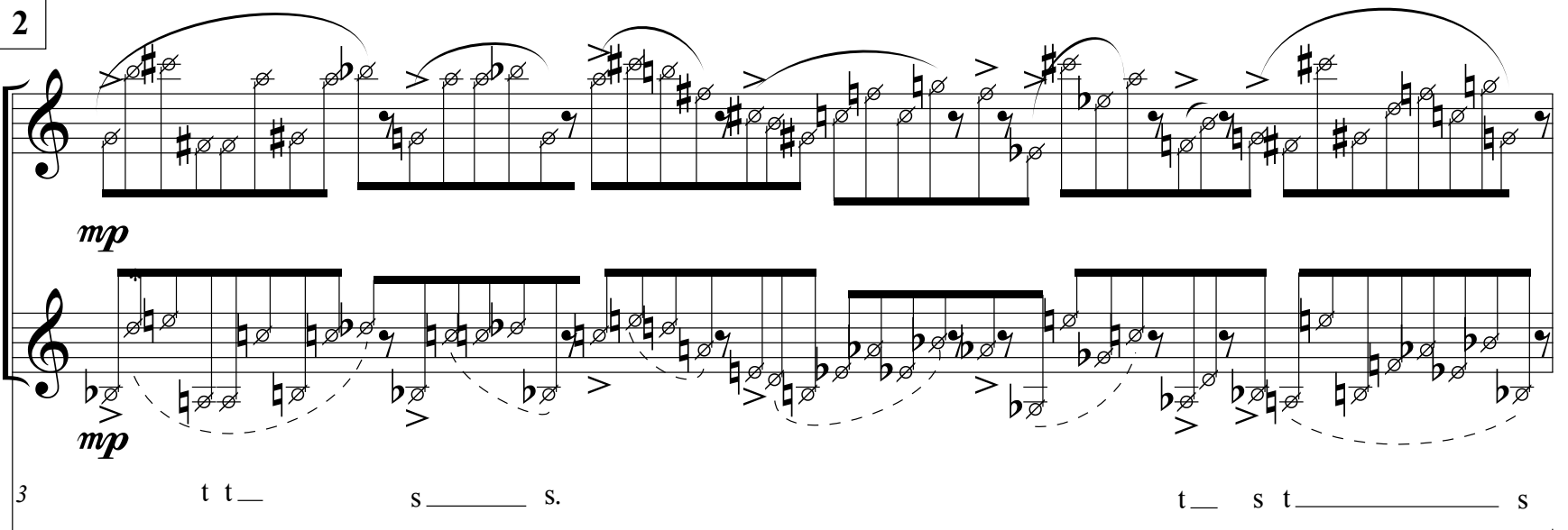

Vox

scattered seeds.

Each morning

I wake to starlings

$\mathrm{B} b \mathrm{Cl} .1$

Fl.

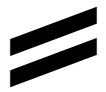$$
\text { - }
$$$$
\text { (n) }
$$

$$
\text { (a) }
$$

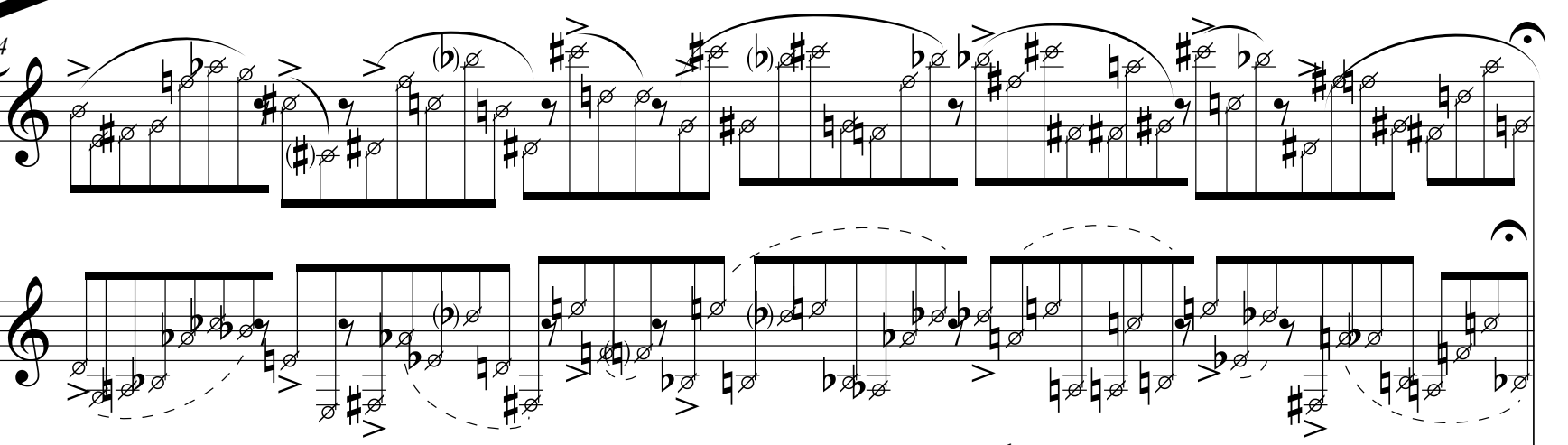

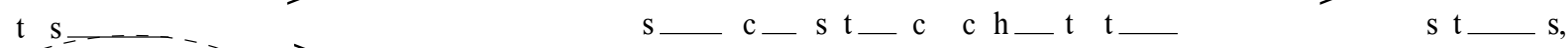

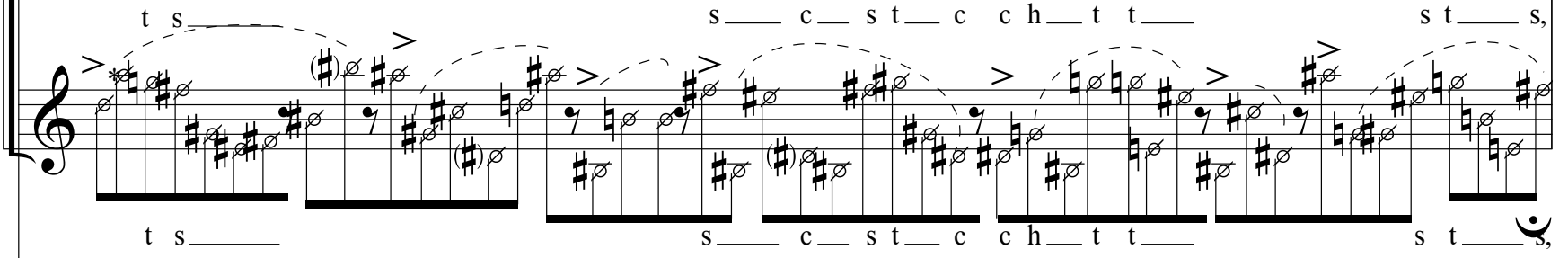

B b Cl. 2

4

Vox

* Play directly into mouthpiece or bocal without reed. Slash noteheads indicate key clicks. Percussively accent where indicated. Insert intersitical contiguous main body keys as gracce notes where possible ad libitum. Text undeneath indicates vocalization. Extended lines indicate a sustain of the frictive noise component

** Key clicks should be very loud in comparison to the vocal sounds. 
Charm Against Loneliness

3

Fl.

$\mathrm{B} b \mathrm{Cl} .1$

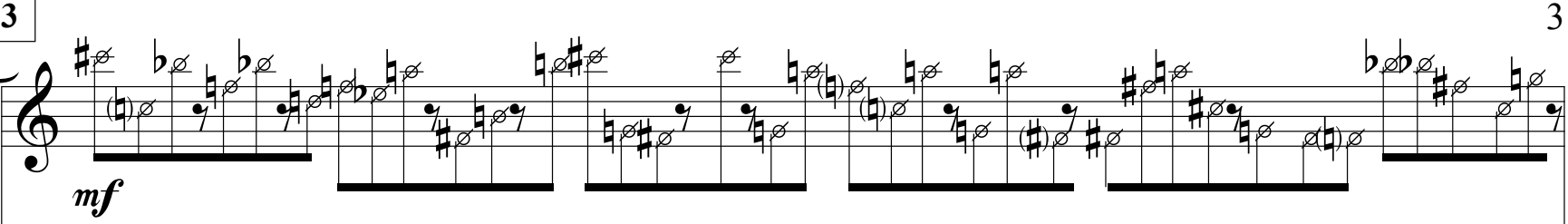

$\mathrm{B} b \mathrm{Cl} .2$

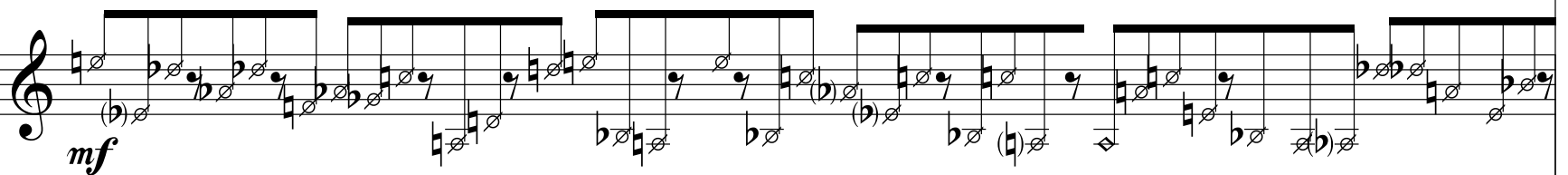

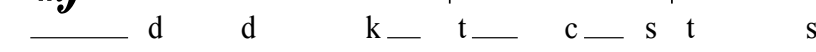

$-\mathrm{s} \_\mathrm{t} \quad \mathrm{h}$

_ $\mathrm{s}$ t_ $\mathrm{d} \mathrm{d}$

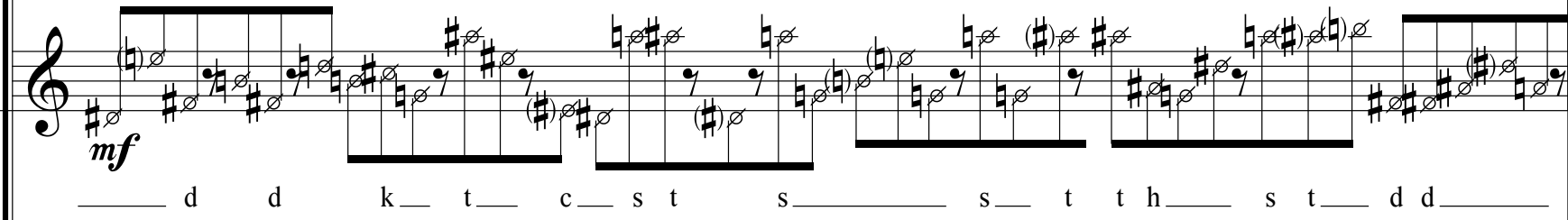

Bsn.
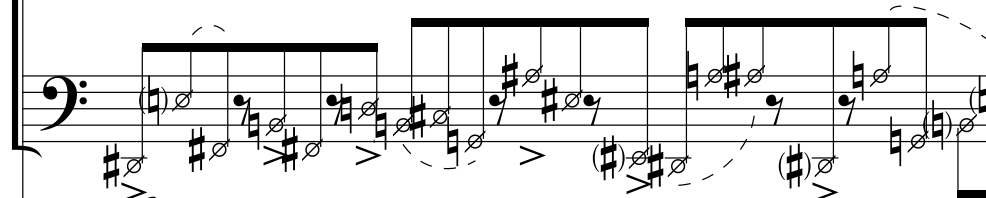

$m f$

$\mathrm{c}$ _ $\mathrm{s} \mathrm{t}$

$\mathrm{S}$

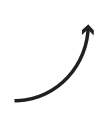

$\boldsymbol{m} \boldsymbol{f}$
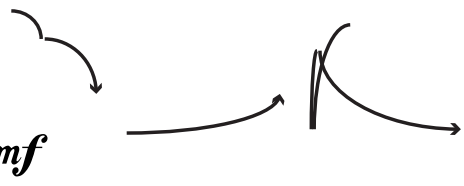

and I'd like to cast

a seine,
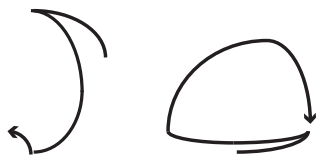

$\longleftarrow$
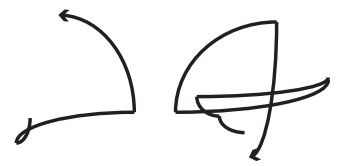

Vox

$\square$

set them studding

*Trace out indicated shapes with the end of a drumstick on the cymbal. 
Charm Against Loneliness

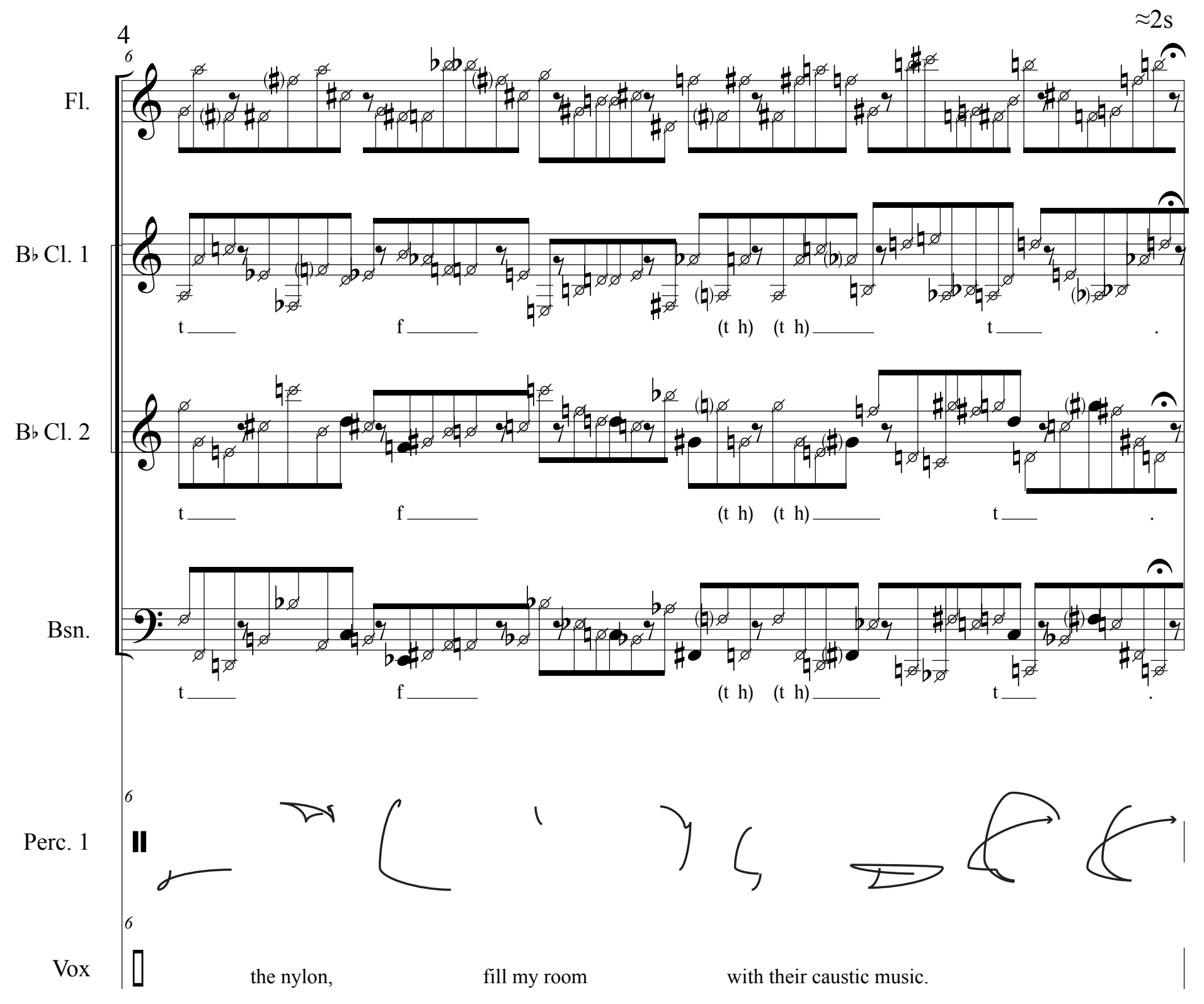




\section{4}

Fl.

B b Cl. 1

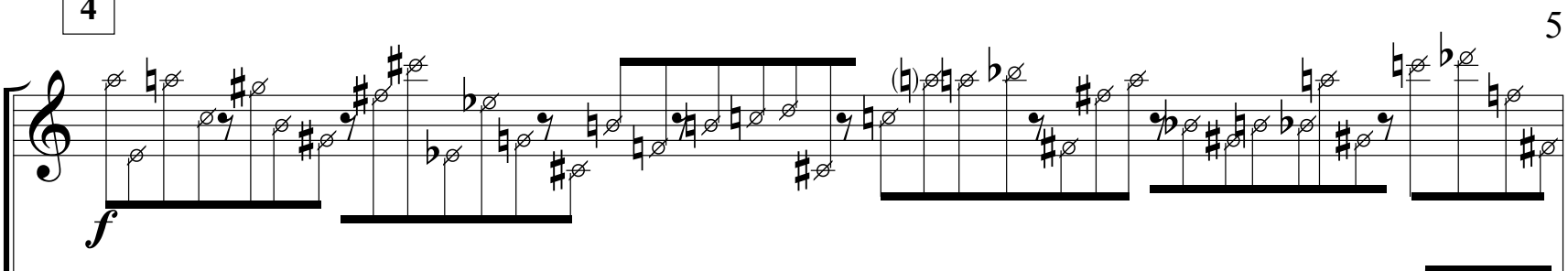

$\mathrm{B} b \mathrm{Cl} .2$

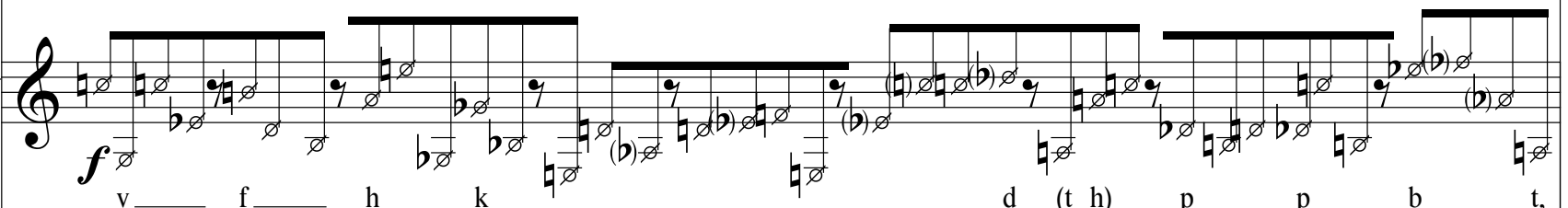

$\mathrm{v}$

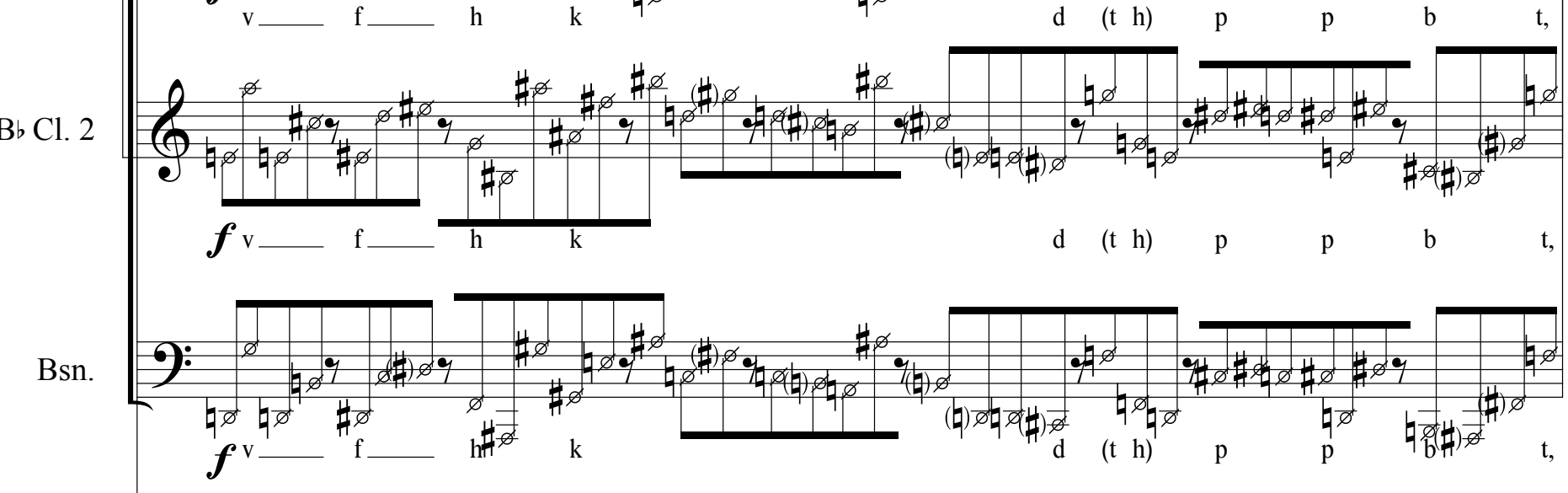

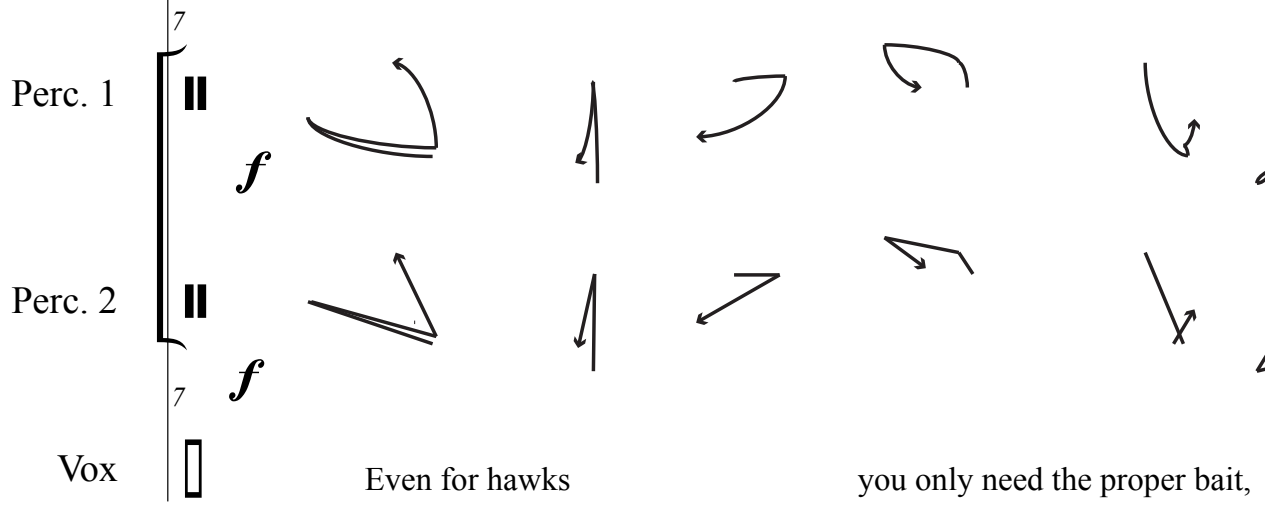

* Trace out indicated shapes with hand on the drumhead. 
Fl.

6

B b Cl. 1

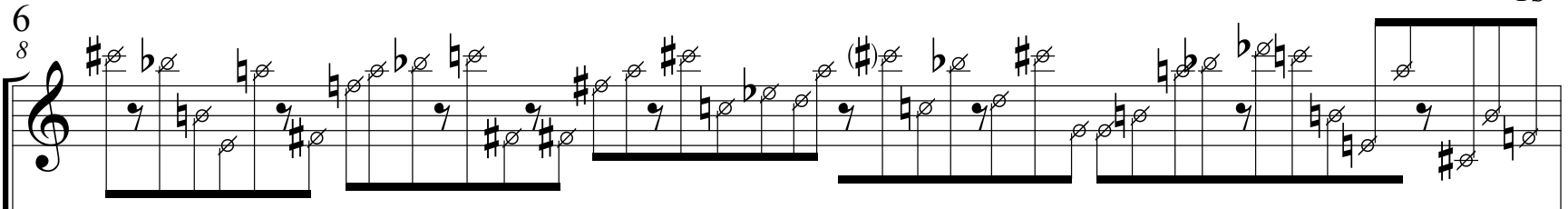

Bb Cl. 2

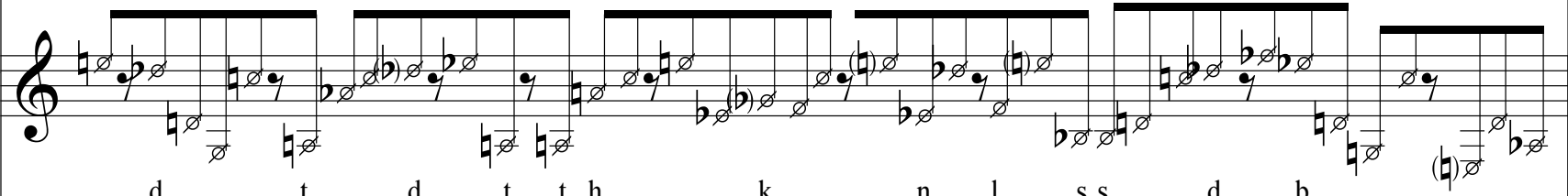

- d

$\mathrm{t}$

$\mathrm{k}$

b

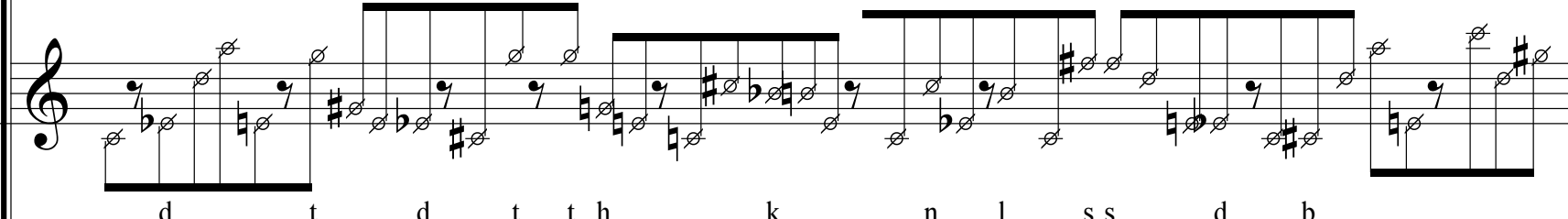

d $\quad t \quad t h$

$\mathrm{k}$

$\mathrm{b}$

Bsn.

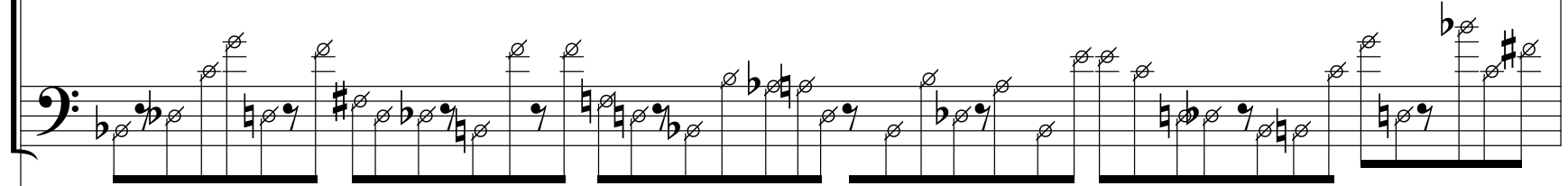

Tuba

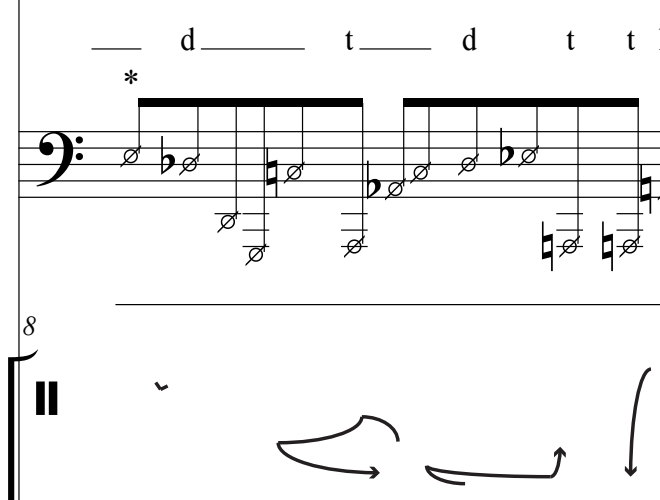

Perc. 2

II

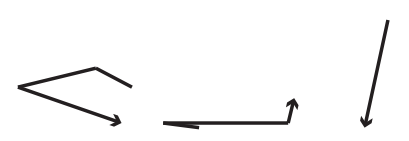

Vox

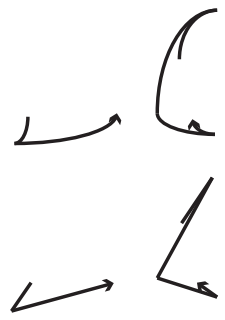

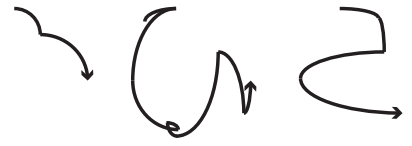

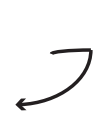

$\searrow$<smiles>CCCCCCC</smiles>

and lassoed above you

* Play without mouthpiece, while still observing the indicated fingerings and by blowing through the horn. The breath sounds are inidcated by the line under the staff. Create a minimum of discrete pitch. Valves can make percussive sounds as well, either by pushing them down rapidly or releasing them quickly and allowing them to rebound. 
Charm Against Loneliness

Fl.

$\mathrm{B} b \mathrm{Cl} .1$

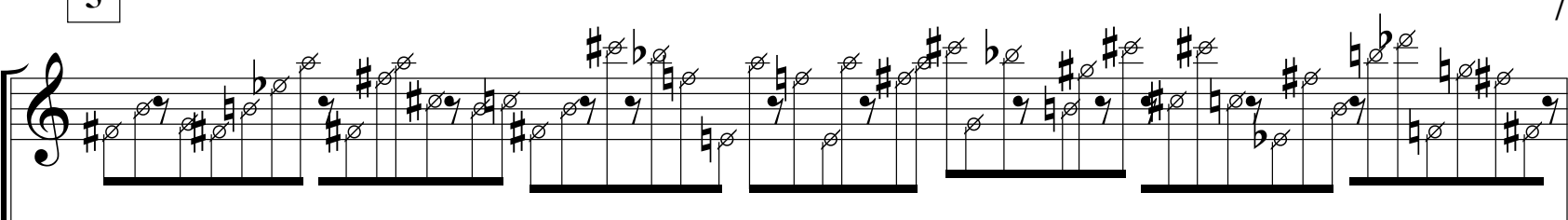

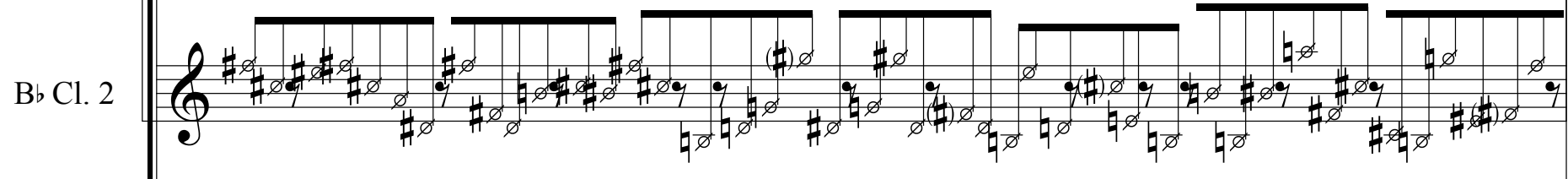

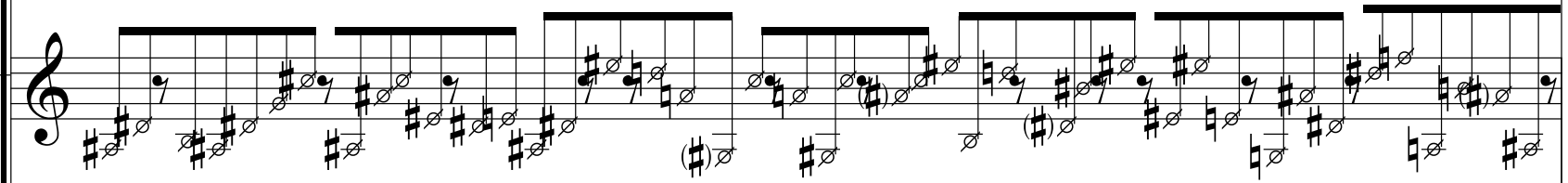

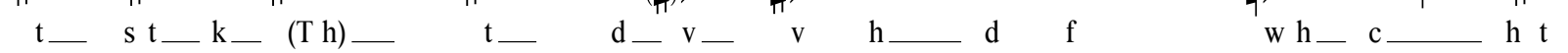

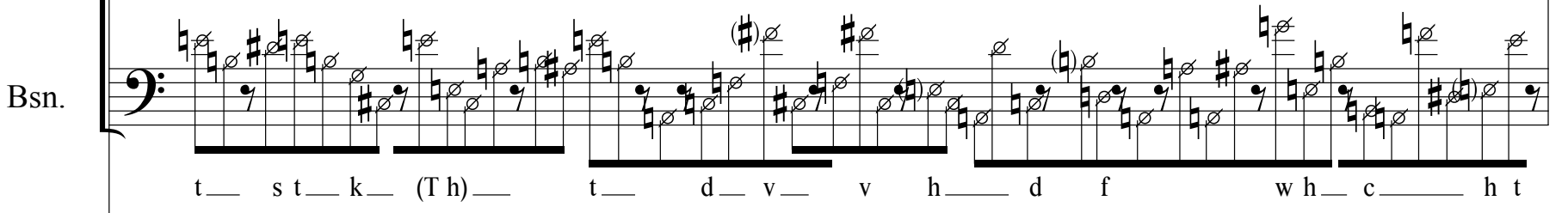

$\mathrm{t} \_\mathrm{st}-\mathrm{k}_{-}(\mathrm{Th}) \mathrm{T}_{-} \mathrm{d} \mathrm{d}_{-} \mathrm{v}_{-} \mathrm{v} \mathrm{h}_{-} \mathrm{d} \quad \mathrm{f} \quad \mathrm{wh}-\mathrm{c}_{-}$ $\mathrm{h} \mathrm{t}$

Tuba
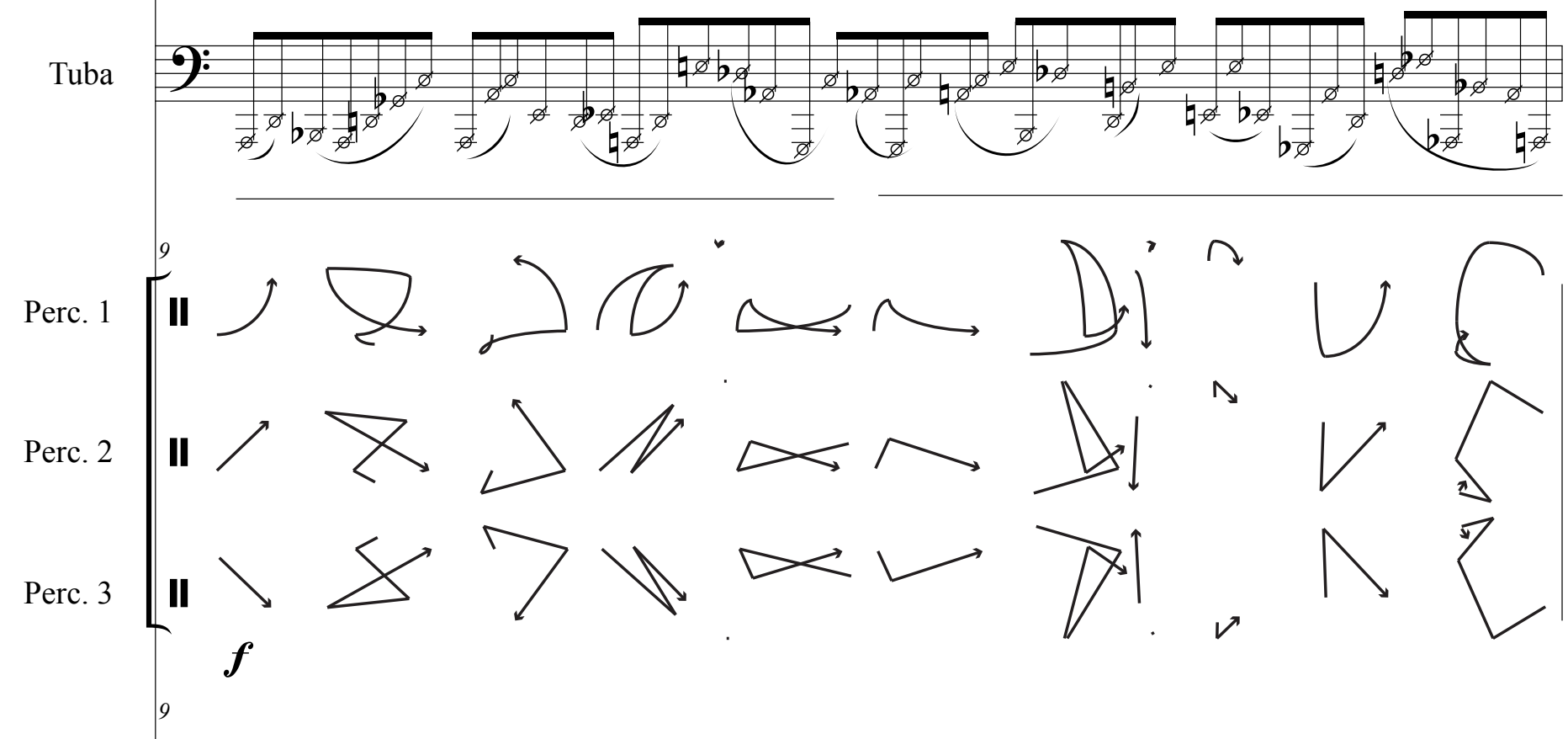

T to stoke them into a dive.

I've heard of a man

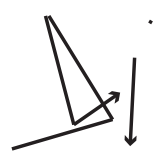

$\searrow$
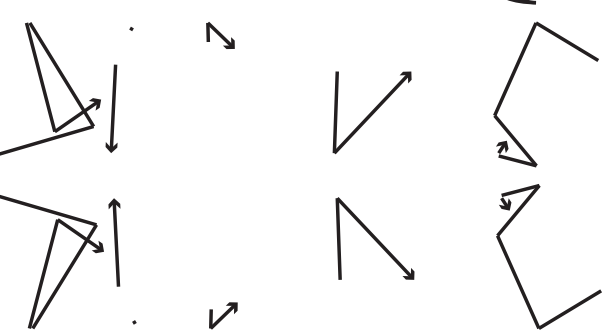

senza arco

Vc.

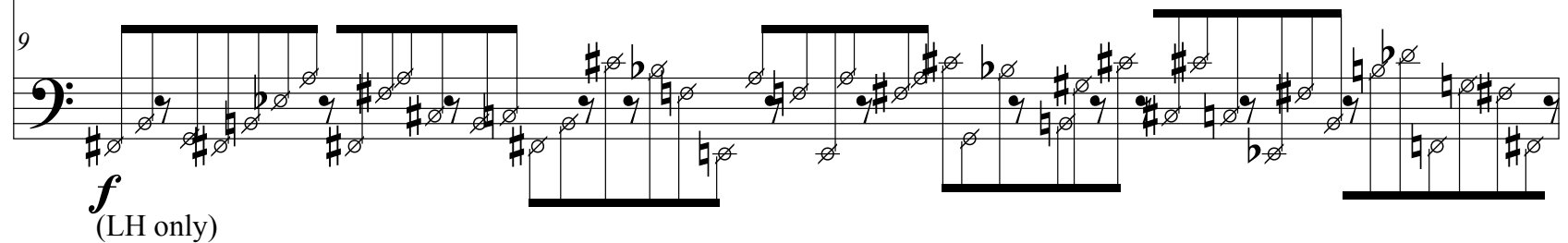

* Trace out indicated shapes with hand on drumhead. 


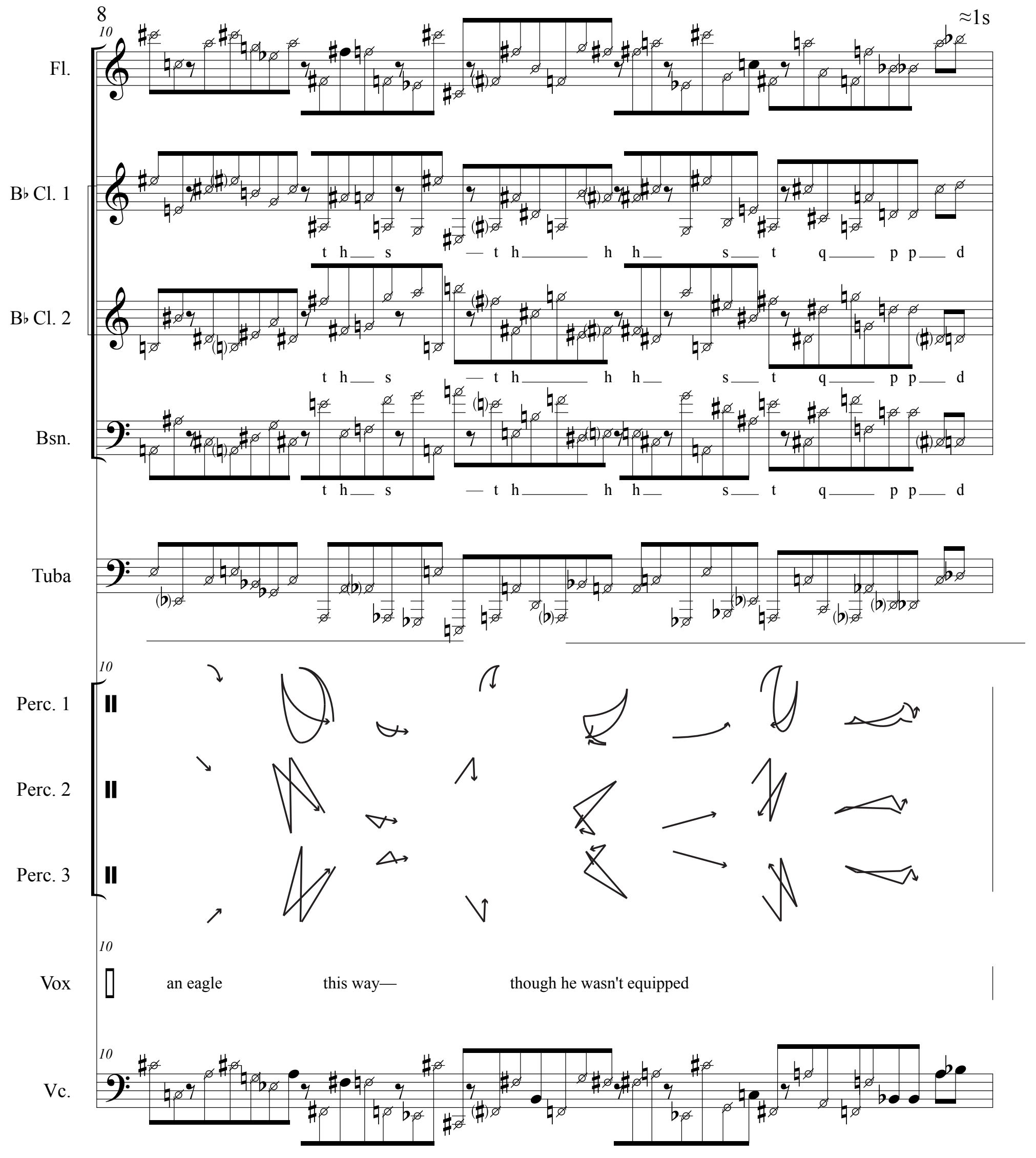


B b Cl. 1

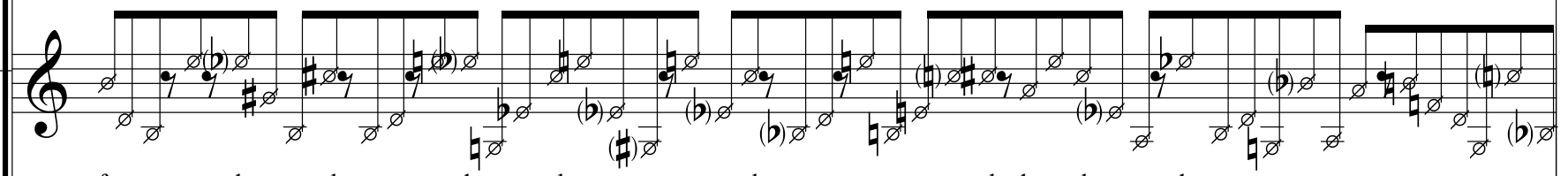
$f \quad r$
$\mathrm{d} s-\mathrm{b}$
d $t$
d $\mathrm{s}$
$\mathrm{d}, \mathrm{h}-\mathrm{d}-\mathrm{t} \quad \mathrm{b}$

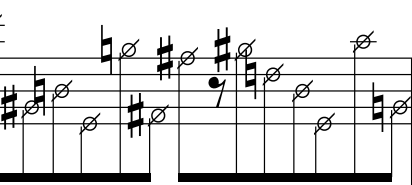

$\mathrm{B} b \mathrm{Cl} .2$

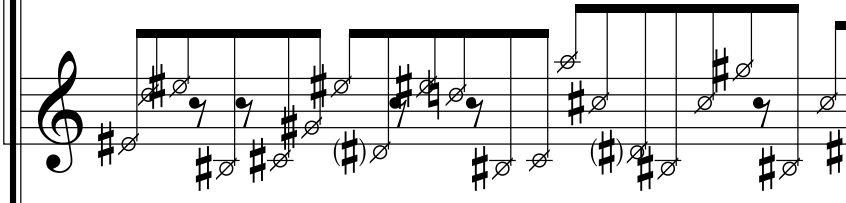

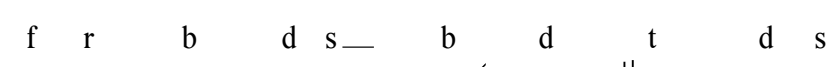

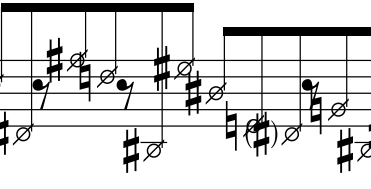

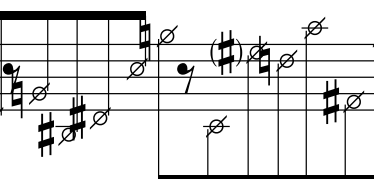

$\mathrm{d}, \mathrm{h}_{-} \mathrm{d} \mathrm{d}_{\mathrm{t}} \mathrm{b} \quad \mathrm{t} \mathrm{g}$

Bsn.

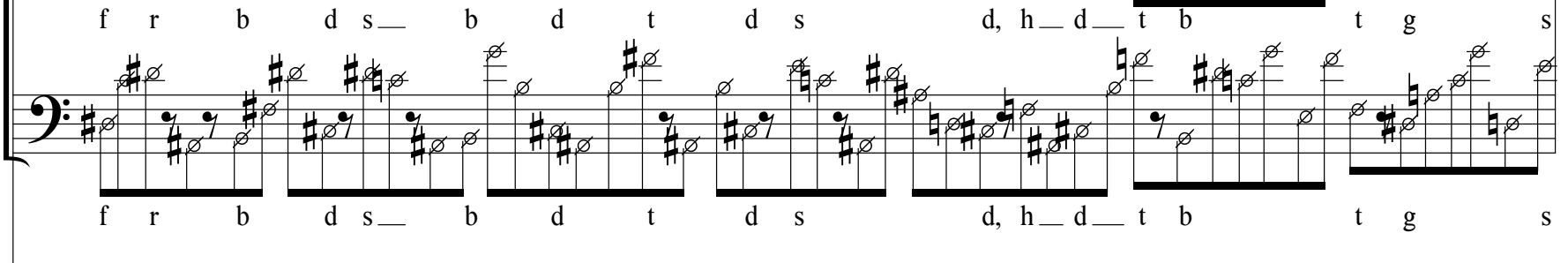

Tuba

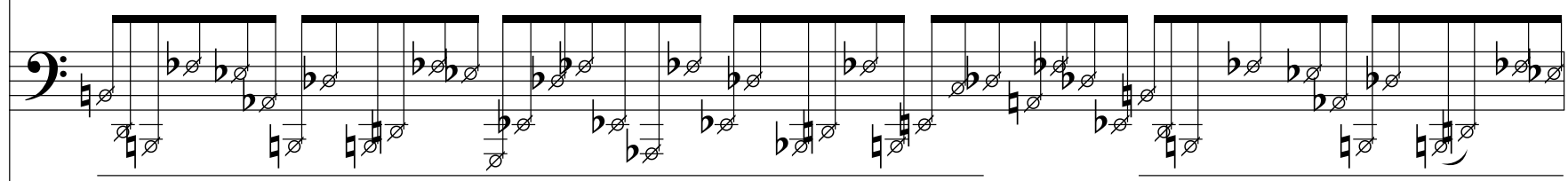

Perc. $1 \llbracket \|$<smiles>CCCC</smiles>

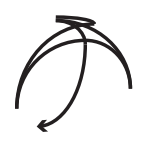

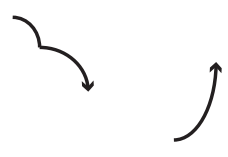

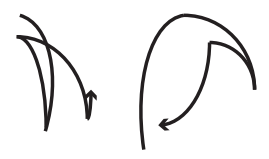<smiles>c1ccccc1</smiles><smiles>C1CCCCC1</smiles>

Perc. 2

Perc. 3

II
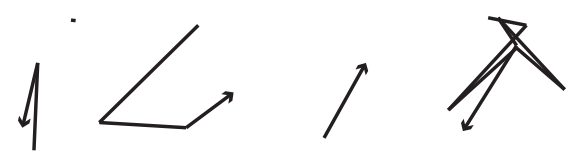

$\searrow$
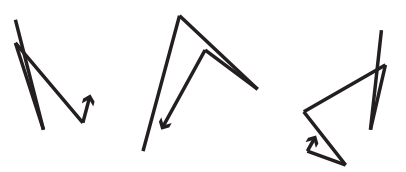

$F$<smiles>CCCCCCC</smiles>

$\searrow$
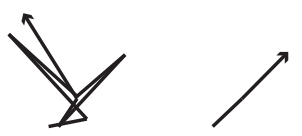

$\searrow$
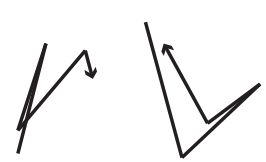

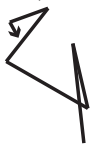

$\$$

Vox $\prod_{11}^{11}$ for a bird so abundant

\& so armed, hadn't brought gloves

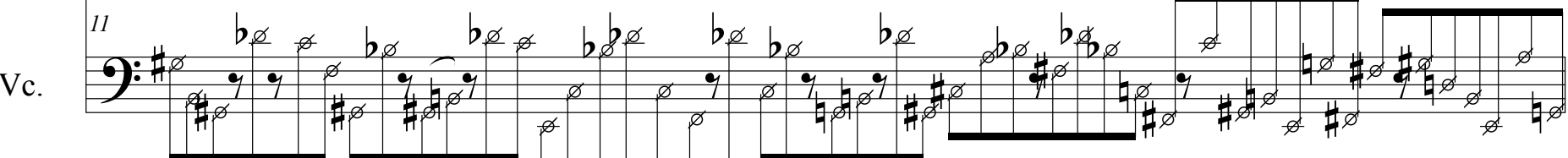


10

F1.

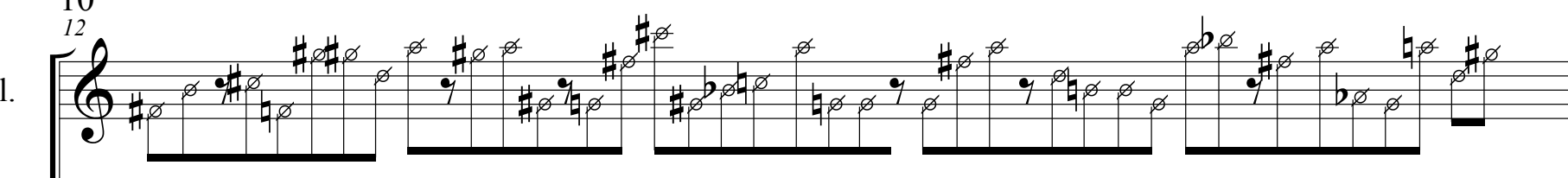

Bb Cl. 1

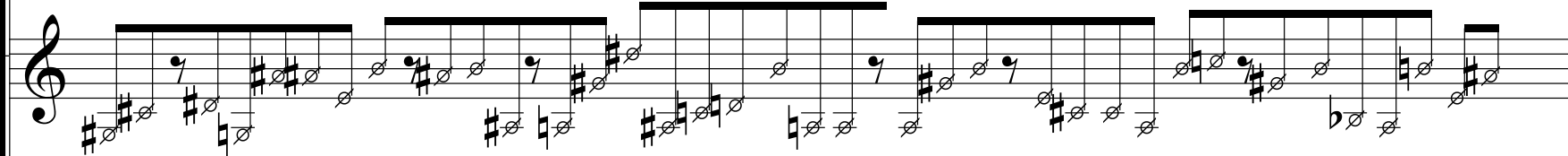
t- $\mathrm{f}$ $\mathrm{s} \mathrm{h}$

$\mathrm{s}-\mathrm{d}$ f.

$\mathrm{B} b \mathrm{Cl} .2$

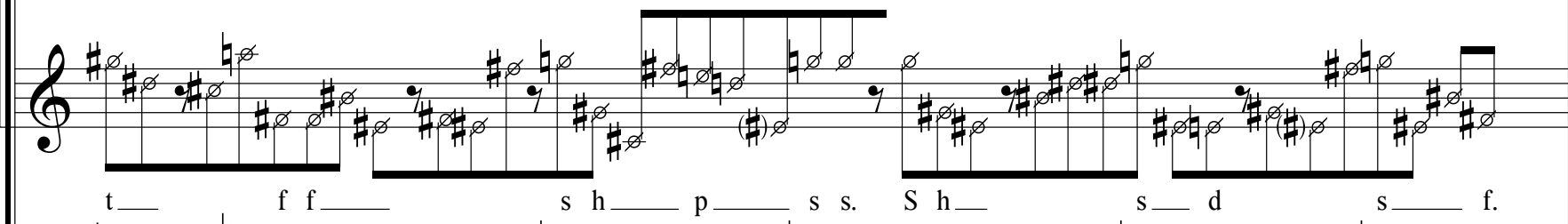

Bsn.

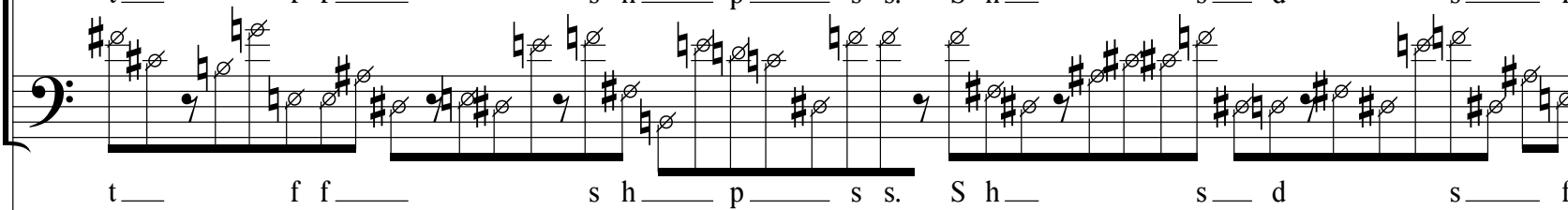

Tuba
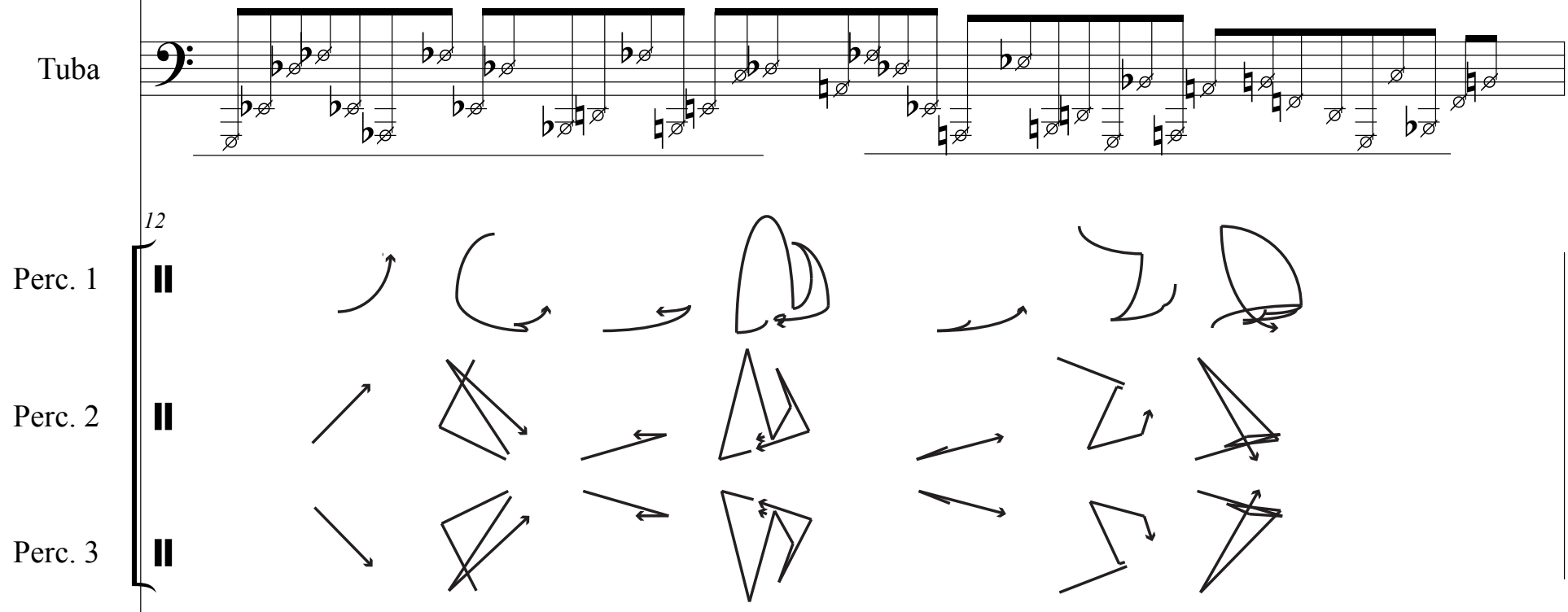

Vox $\quad \prod_{\quad}^{12} \quad$ to muffle her sharpness

She loosed herself.

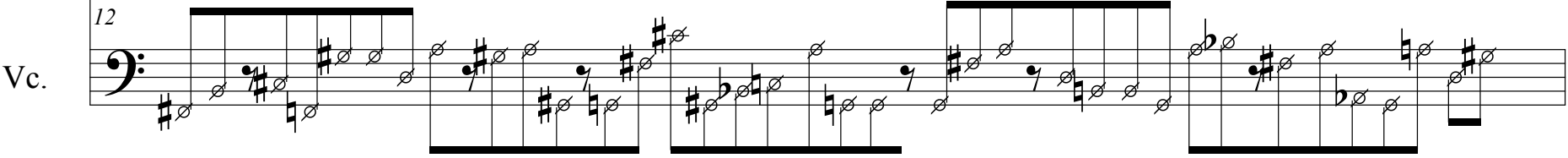




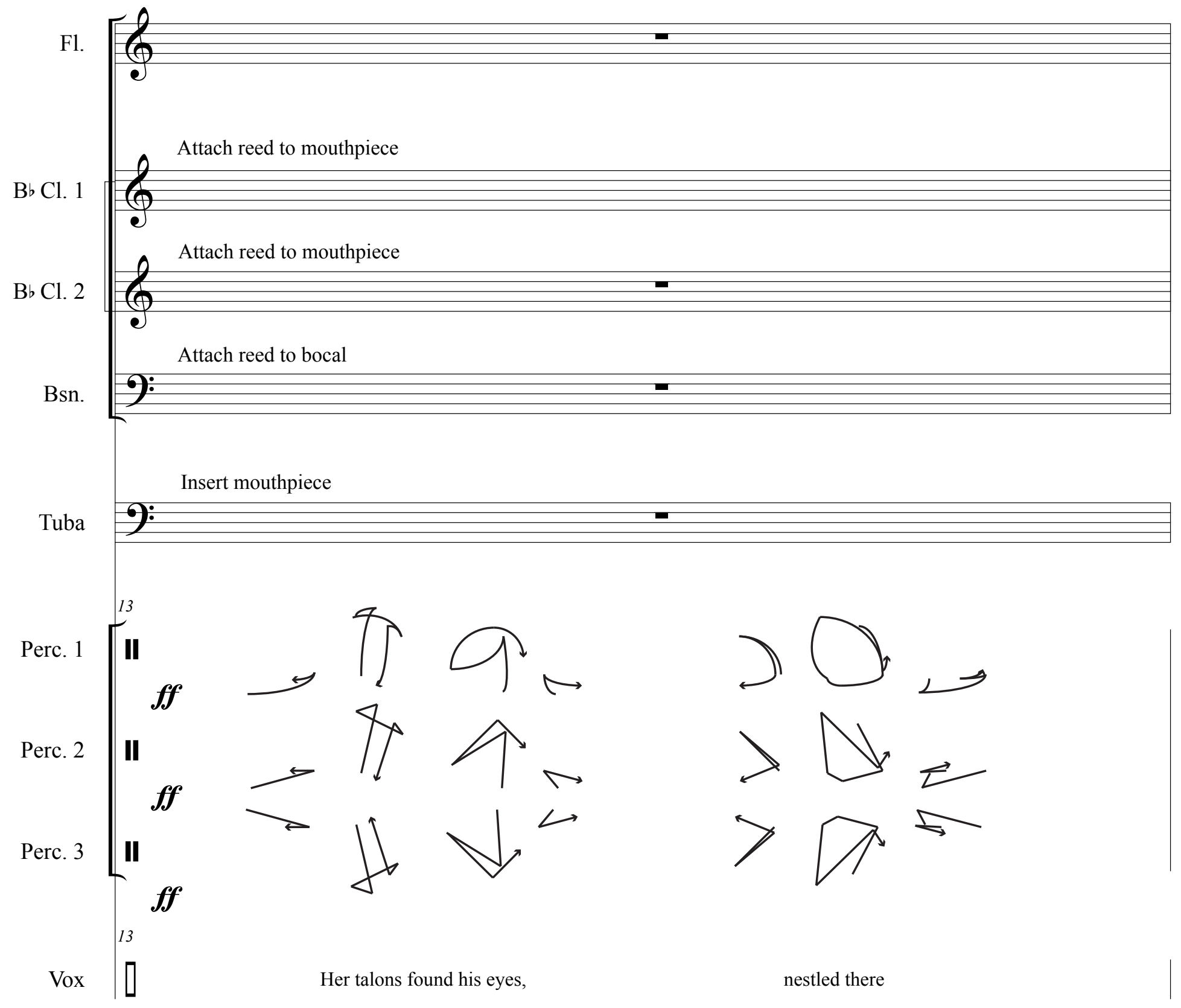




\section{Charm Against Loneliness}

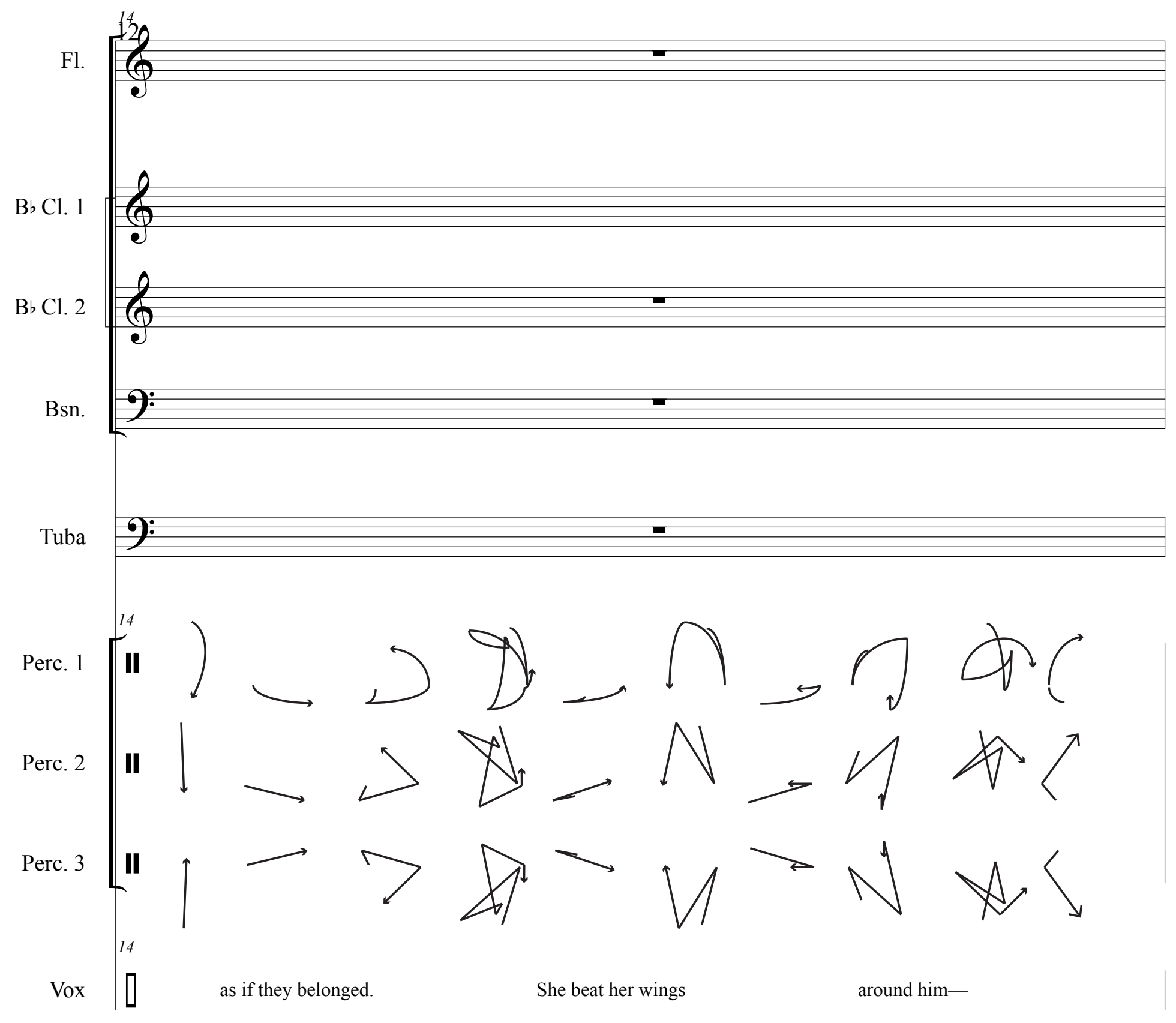


$\mathrm{B} b \mathrm{Cl} .1$

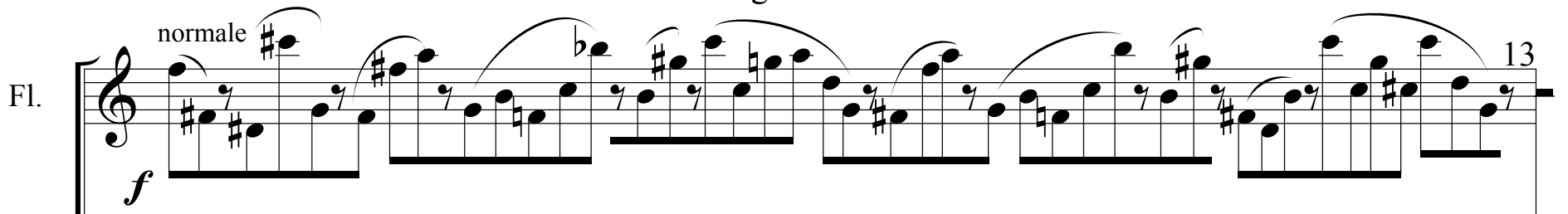

Bb Cl. 2

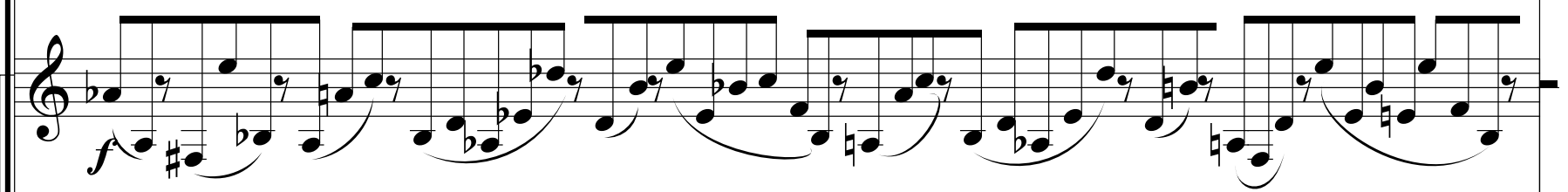

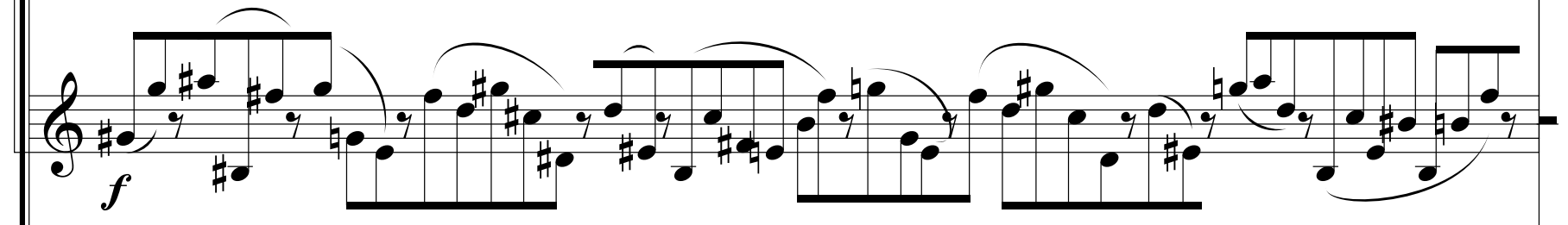

Bsn.

Tuba

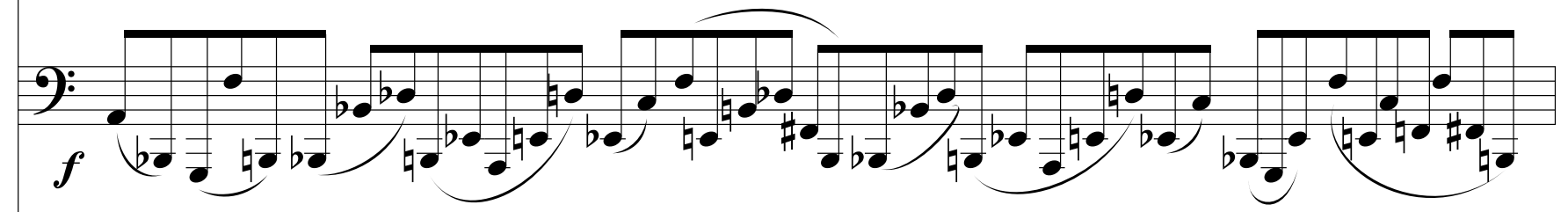

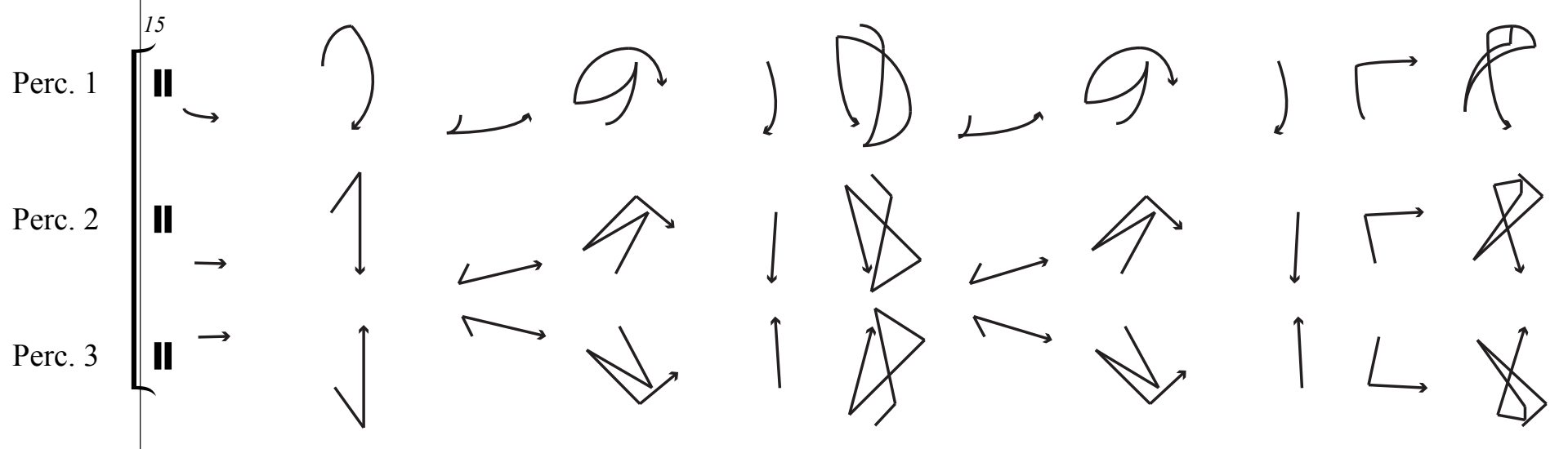

Vox $\prod_{\square}^{15}$ it was the sound of angels,

the sound of two animals

Vc. 
Fl.

B b Cl. 1

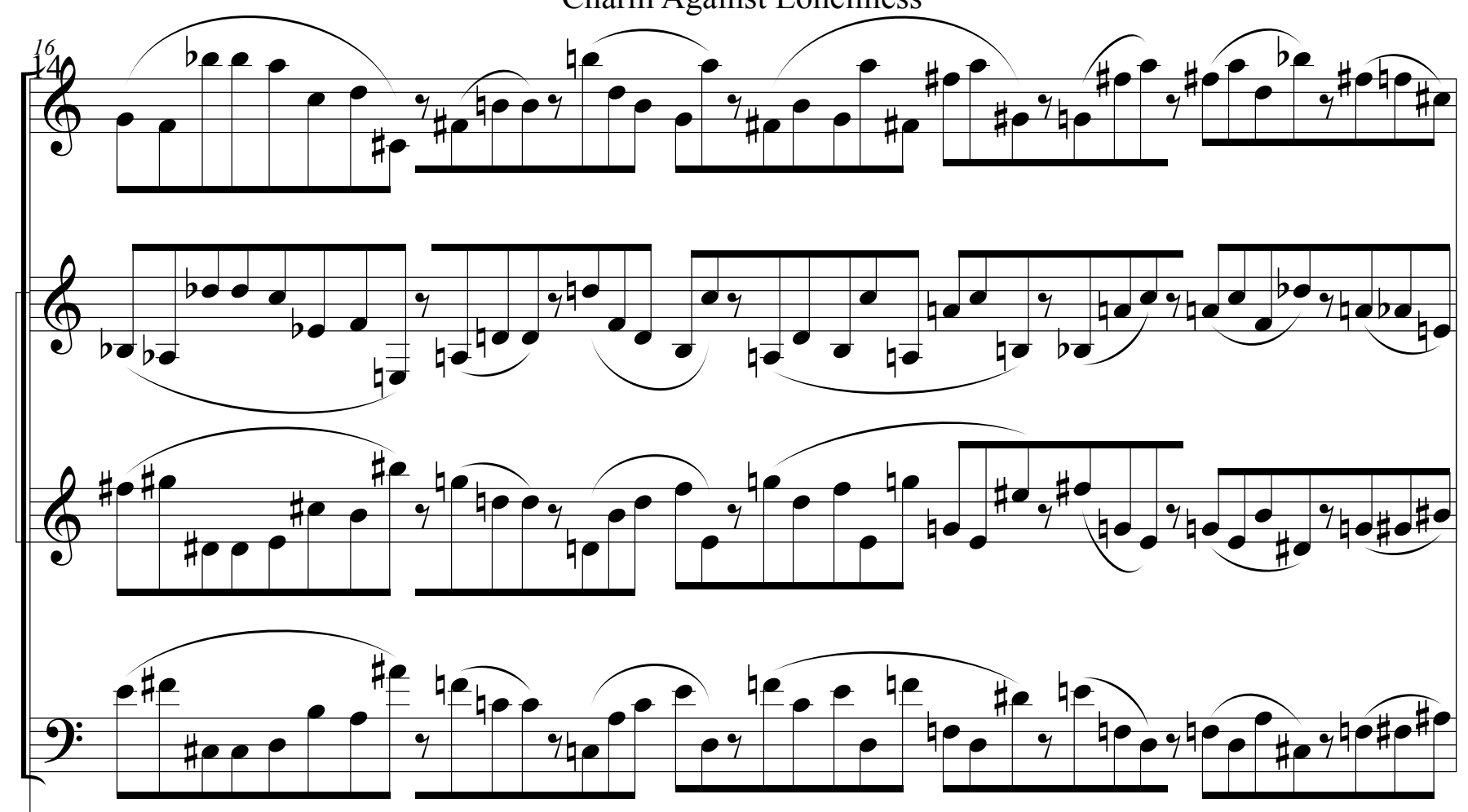

Bb Cl. 2

Bsn.

Tuba
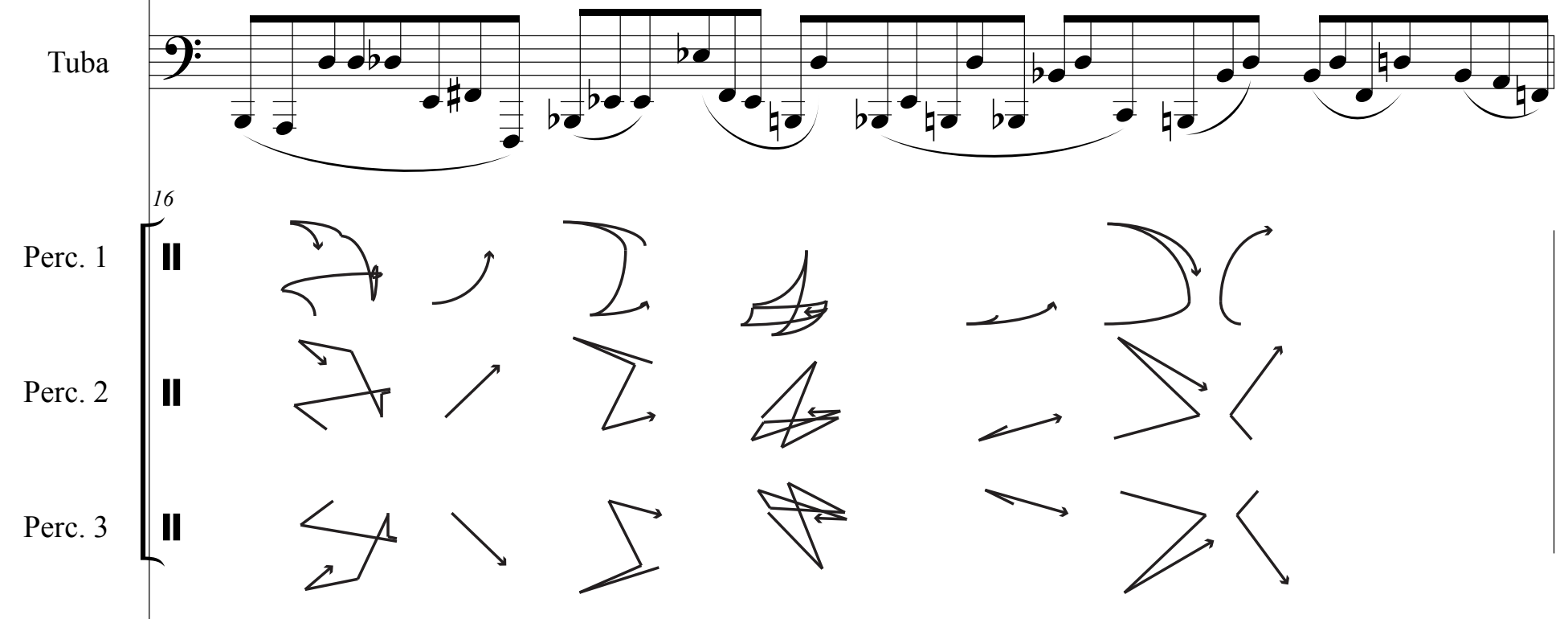

Vox $\quad$ Vc. Suddenly too close held him 


\section{Charm Against Loneliness}

9

Fl.

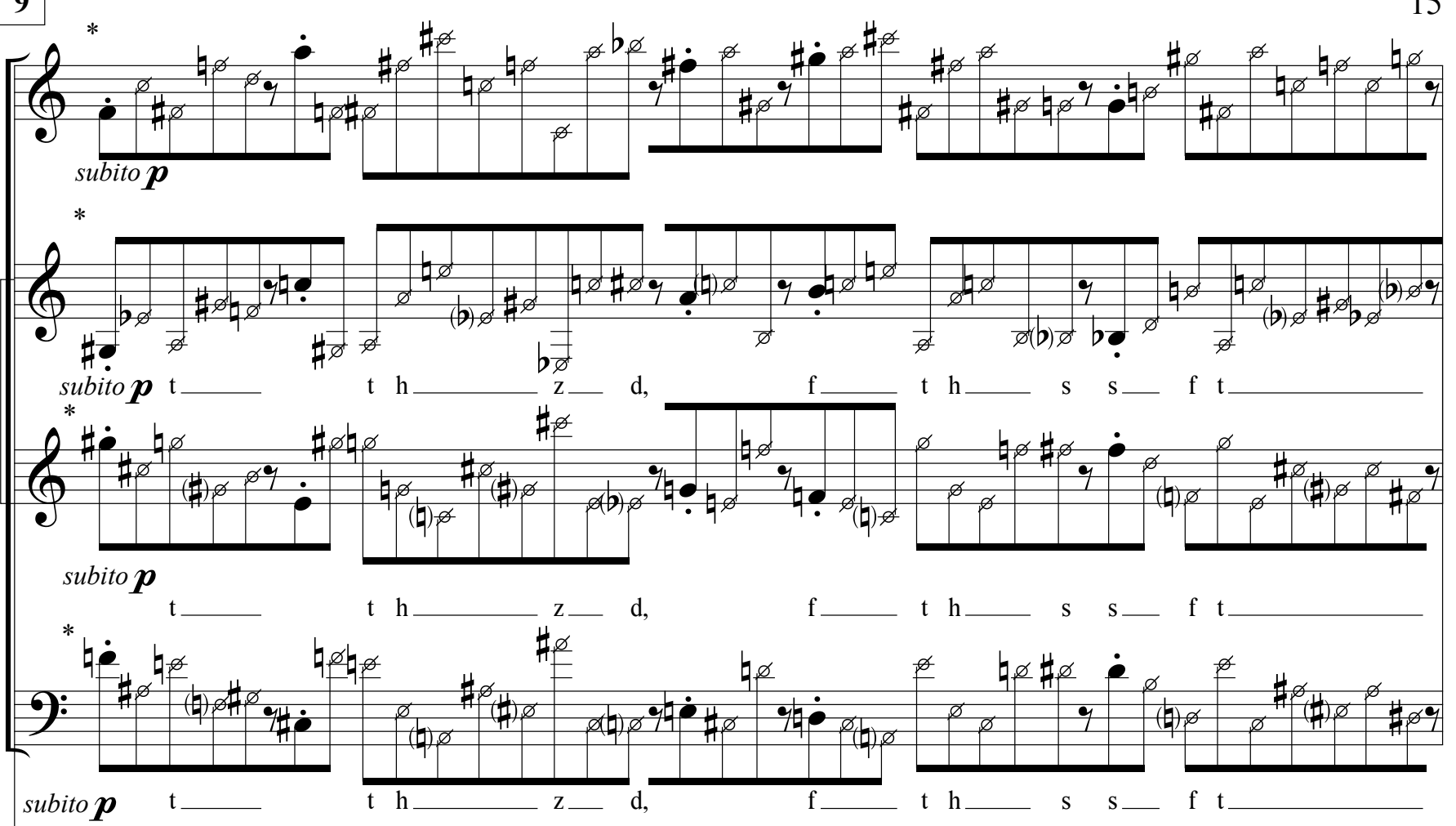

$\mathrm{B} b \mathrm{Cl} .2$

Bsn.

$\mathrm{B} b \mathrm{Cl} .1$

Tuba

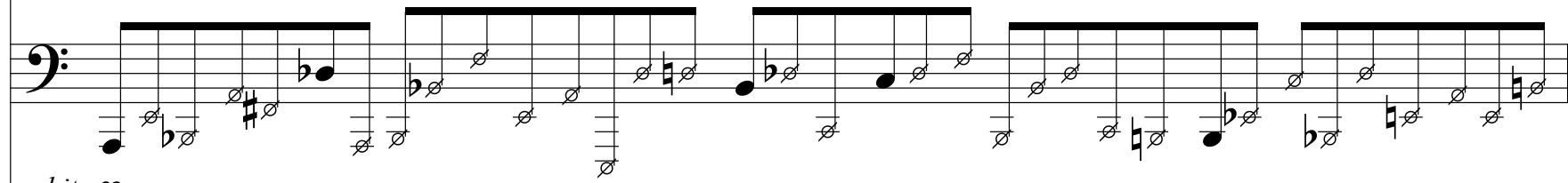

subito $\boldsymbol{p}$

17

Vox

until euthanized, her feathers softening

Vc.

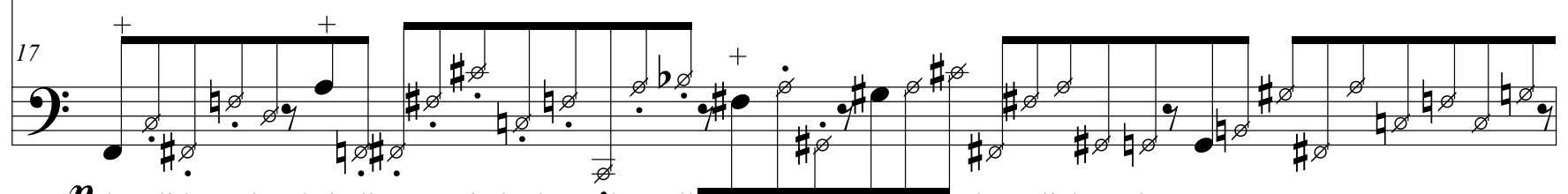

$\boldsymbol{p} *$ Solid noteheads indicate a pitched attack. Hollow noteheads indicate key clicks only.

** Solid noteheads LH attack (senza arco); other noteheads arco, staccato col legno 
Fl.

16

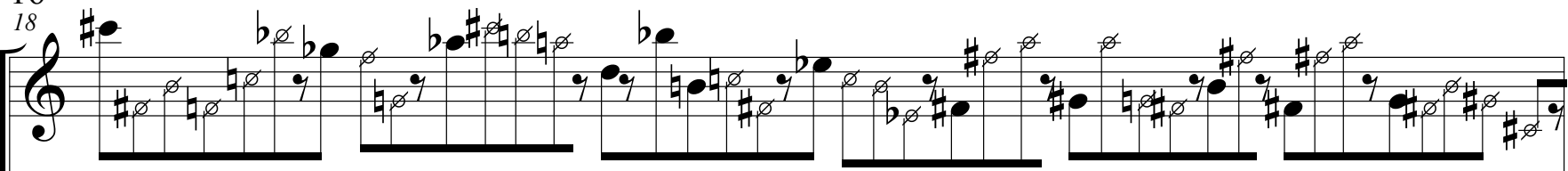

$\mathrm{B} b \mathrm{Cl} .1$

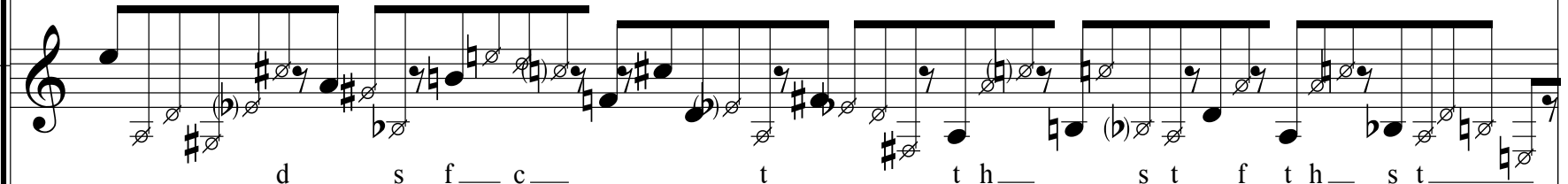

B b Cl. 2
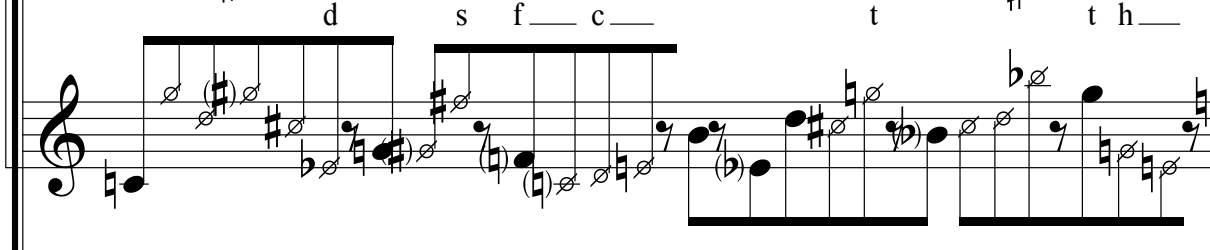

$\mathrm{th}$

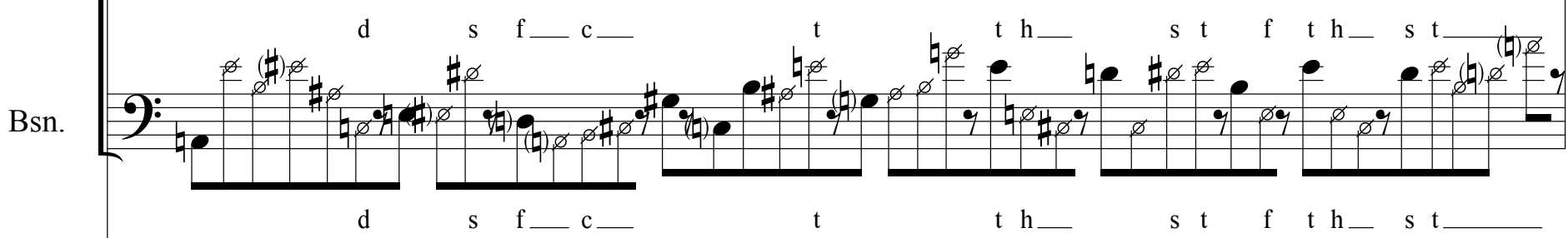

Tuba

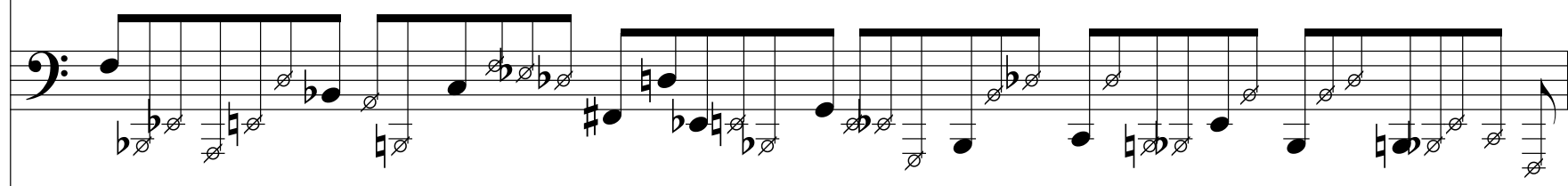

Vox

Vc.

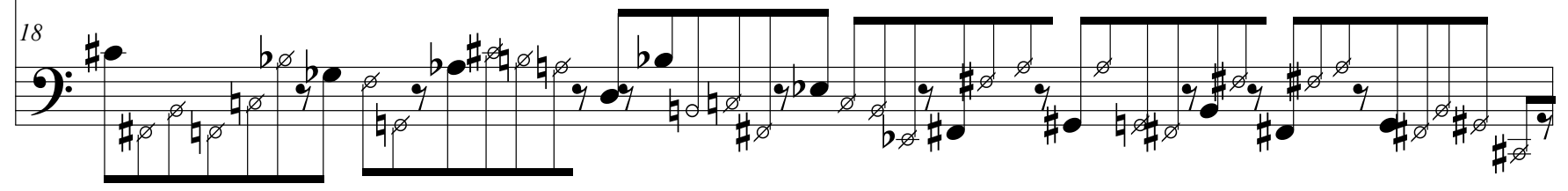


Fl.

Bb Cl. 1

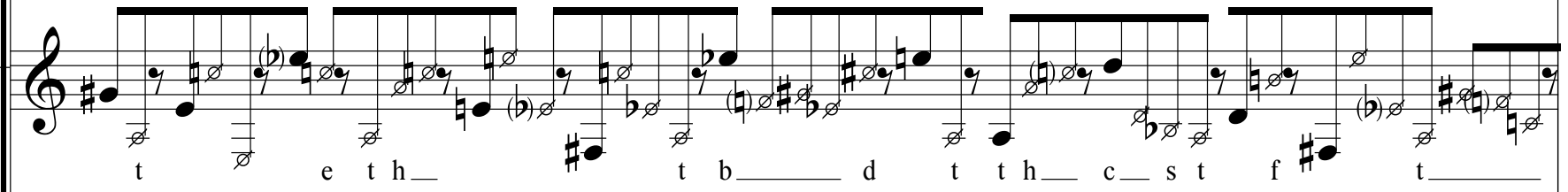

$\mathrm{B} b \mathrm{Cl} .2$

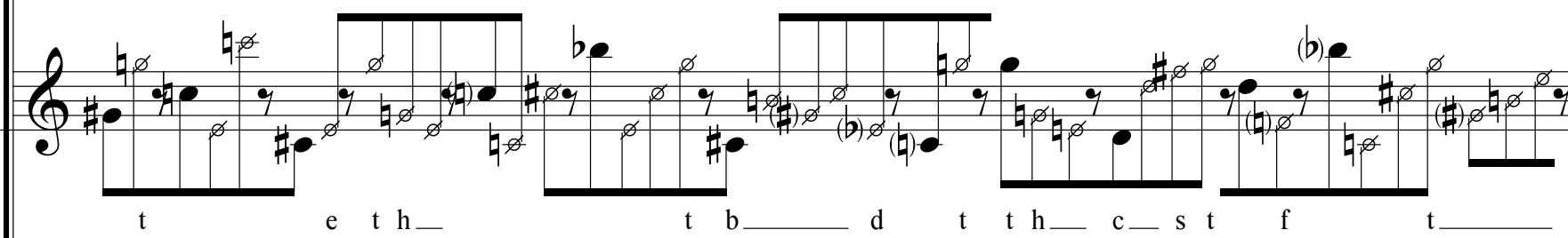

Bsn.

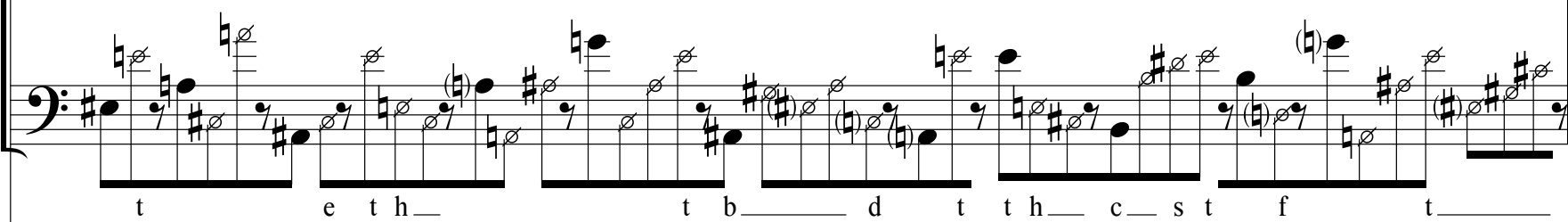

Tuba

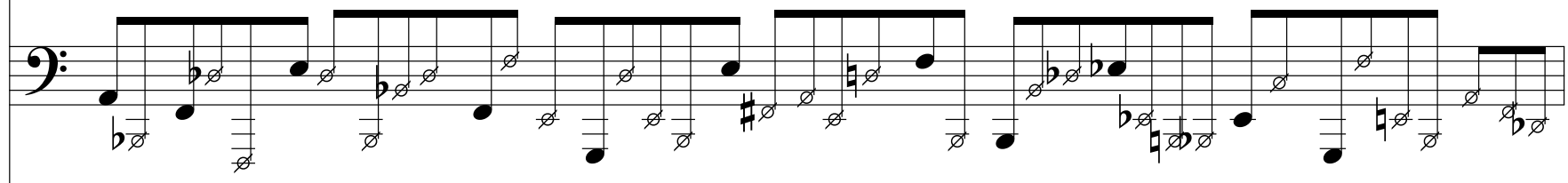

Vox

19

it may be that the man went blind

at the cost

of wanting

Vc.

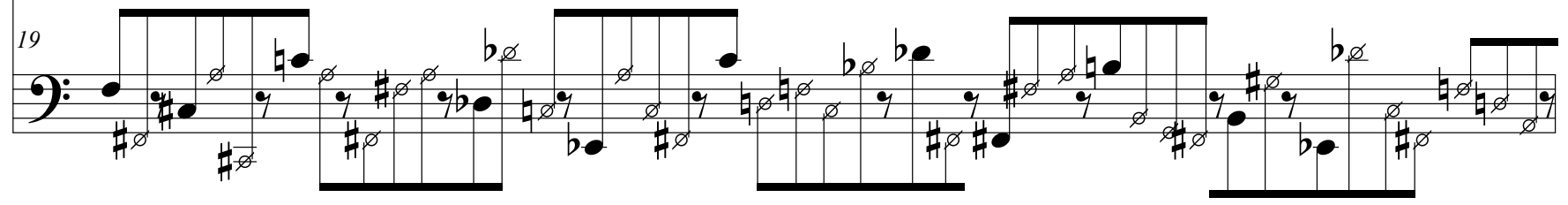




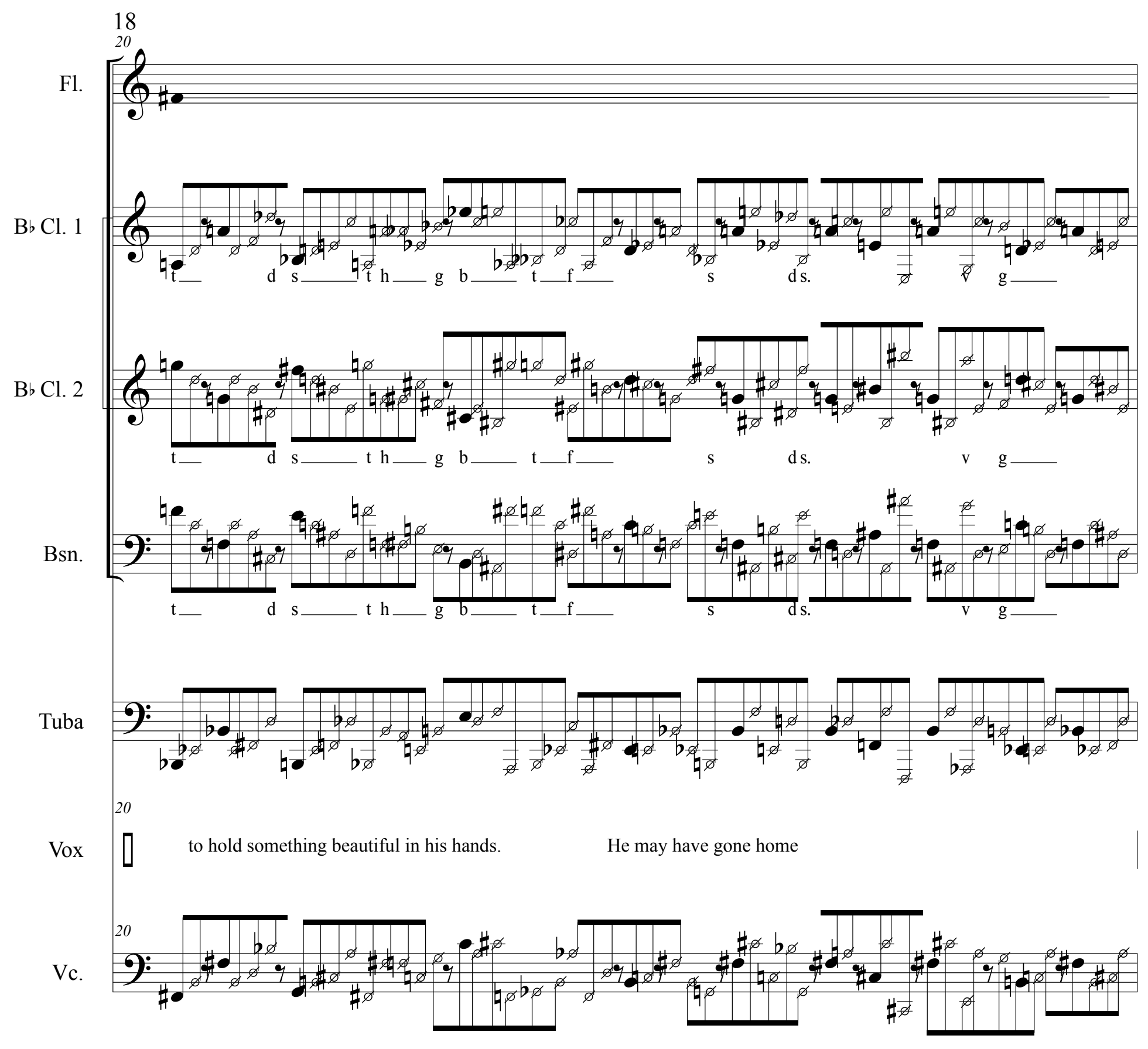




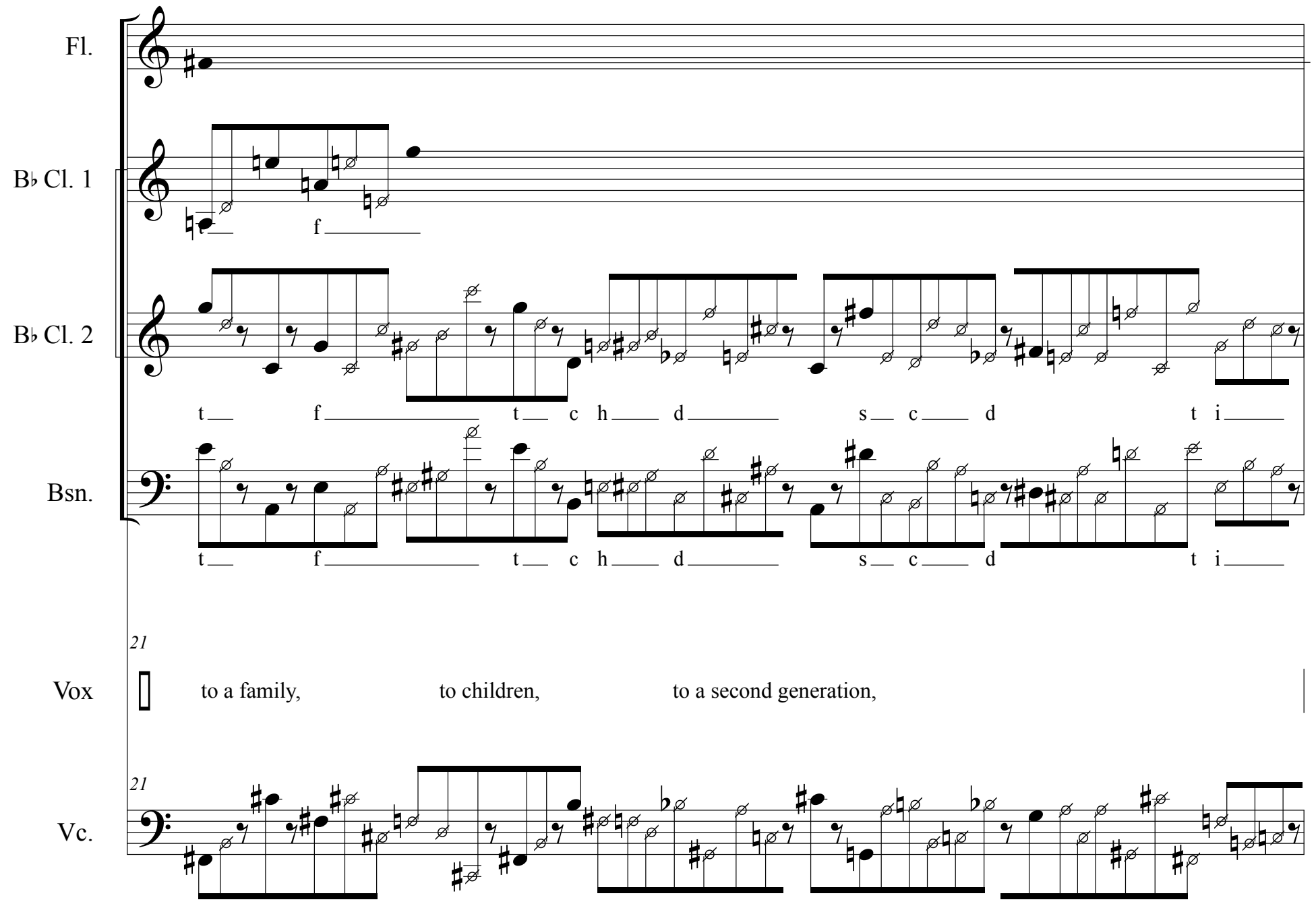


Charm Against Loneliness

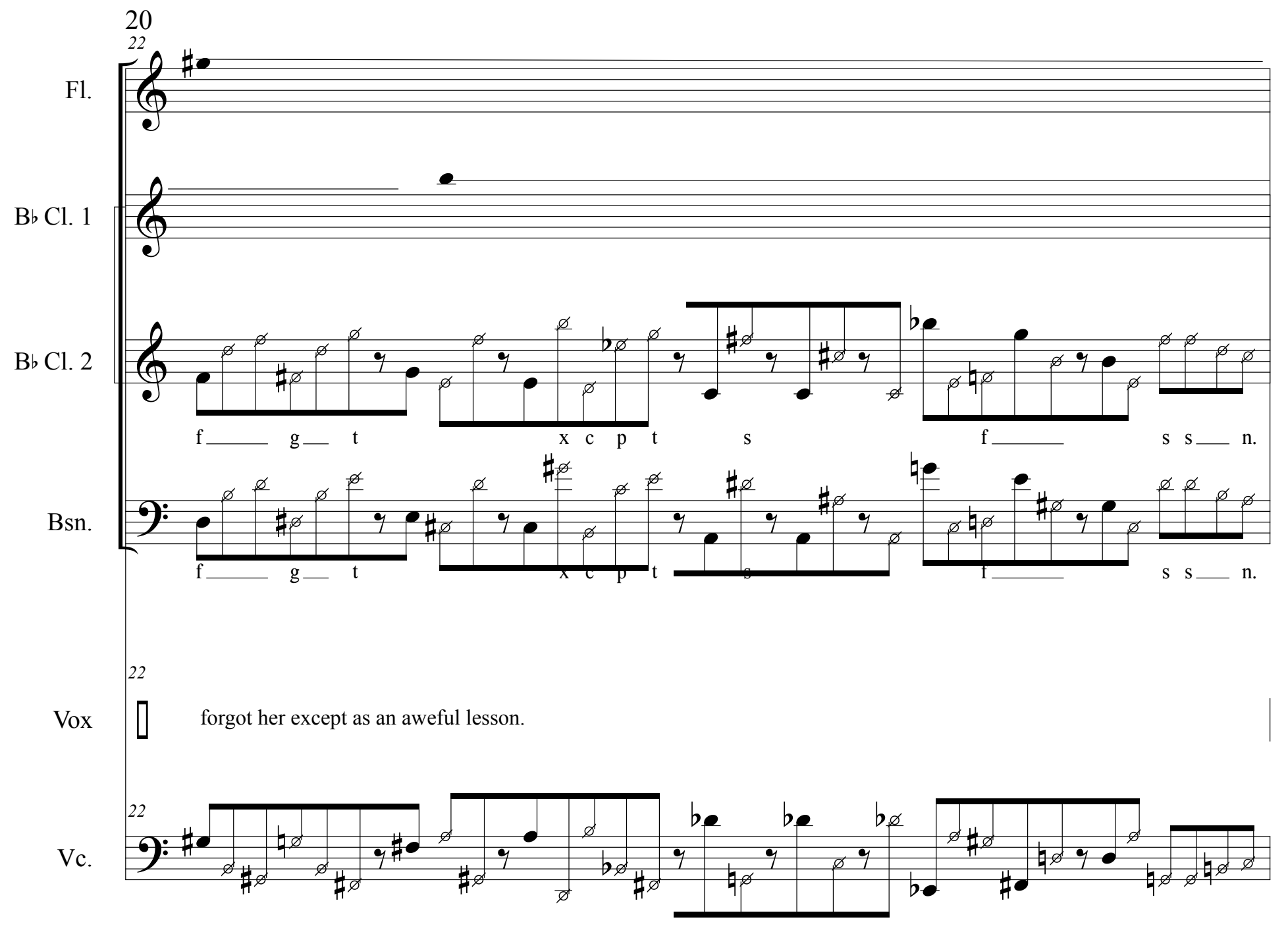


Charm Against Loneliness

12
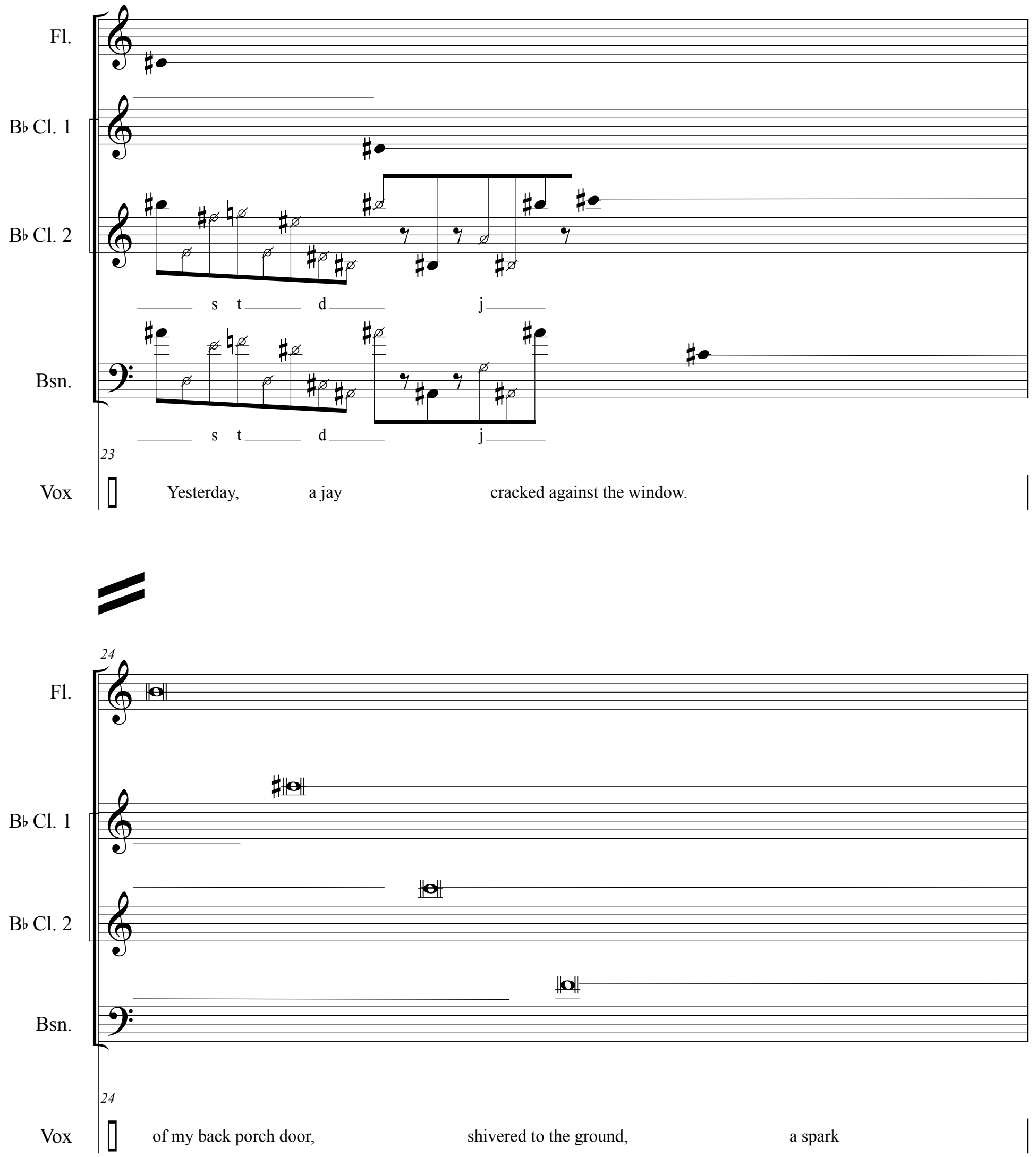
Charm Against Loneliness

23

Fl.

$\mathrm{B} b \mathrm{Cl} .1$

Bb Cl. 2

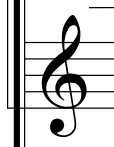

Bsn.

):

\section{$\# \theta$}

Tuba

9:

$\operatorname{Perc.~} 1 \Gamma^{25} \longrightarrow$

Perc. 2 ||

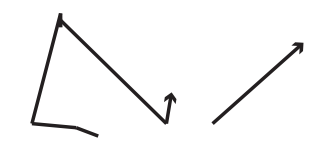

Perc. 3

【
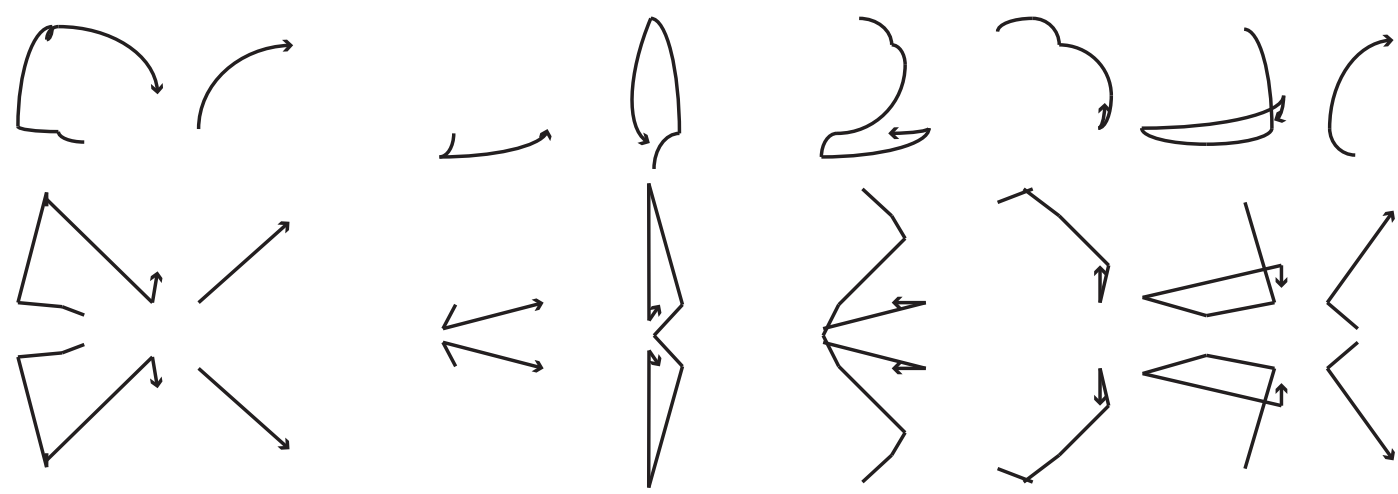

Another landed beside him,

Vox $\square \quad$ stilled in the grass.

Vc.

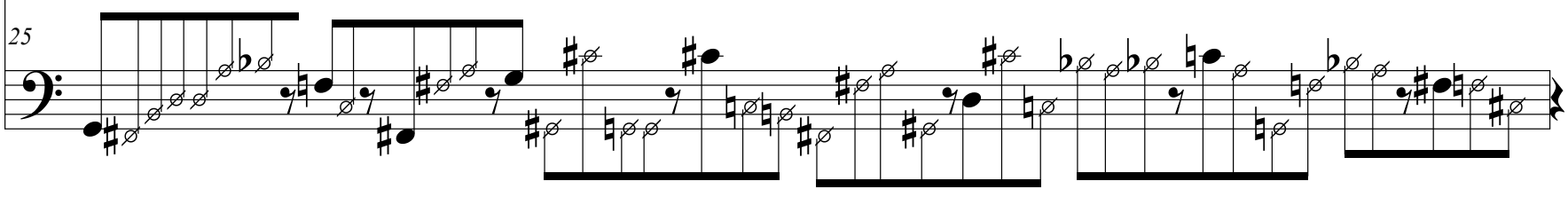

* Articulate notes with solid notehead. Key clicks and breathy half-tone for others. 


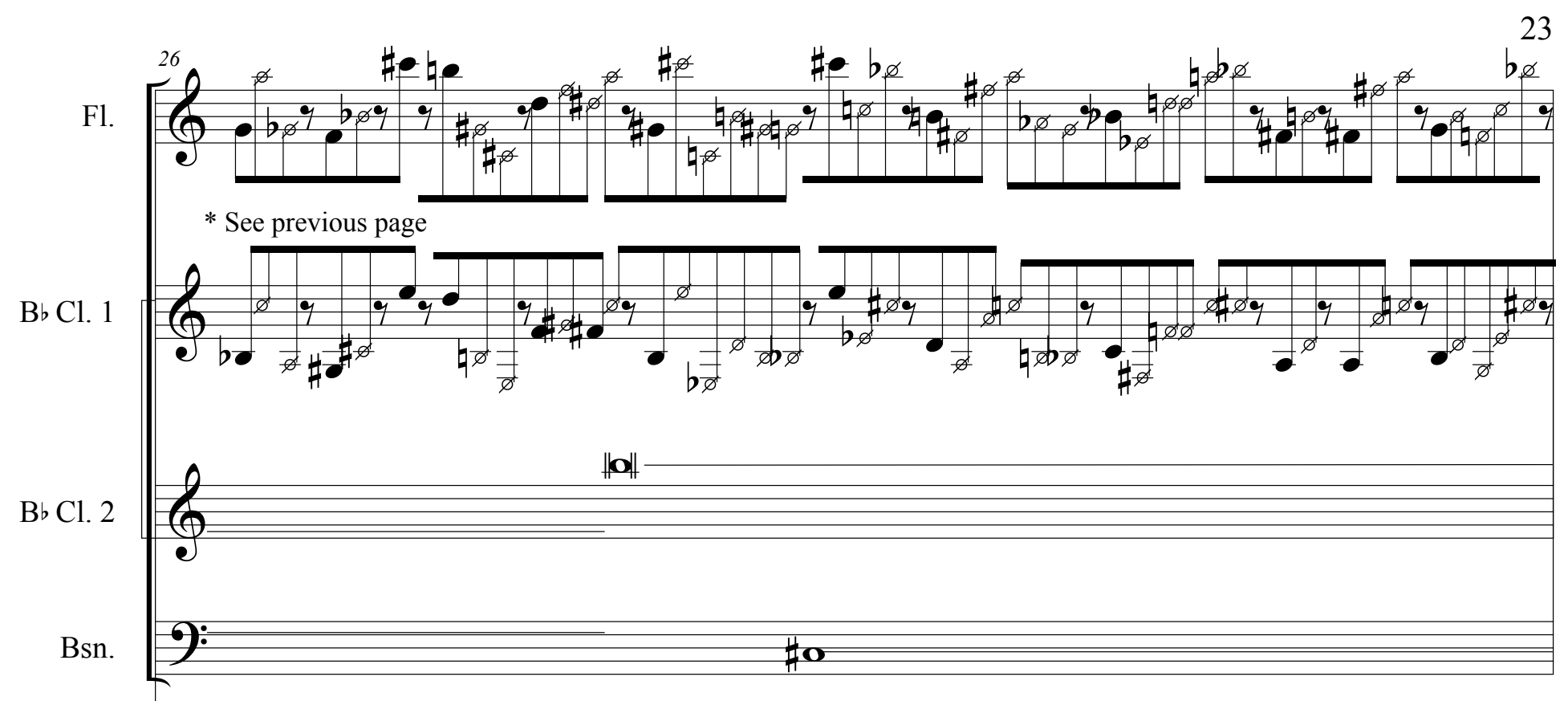

Tuba 9

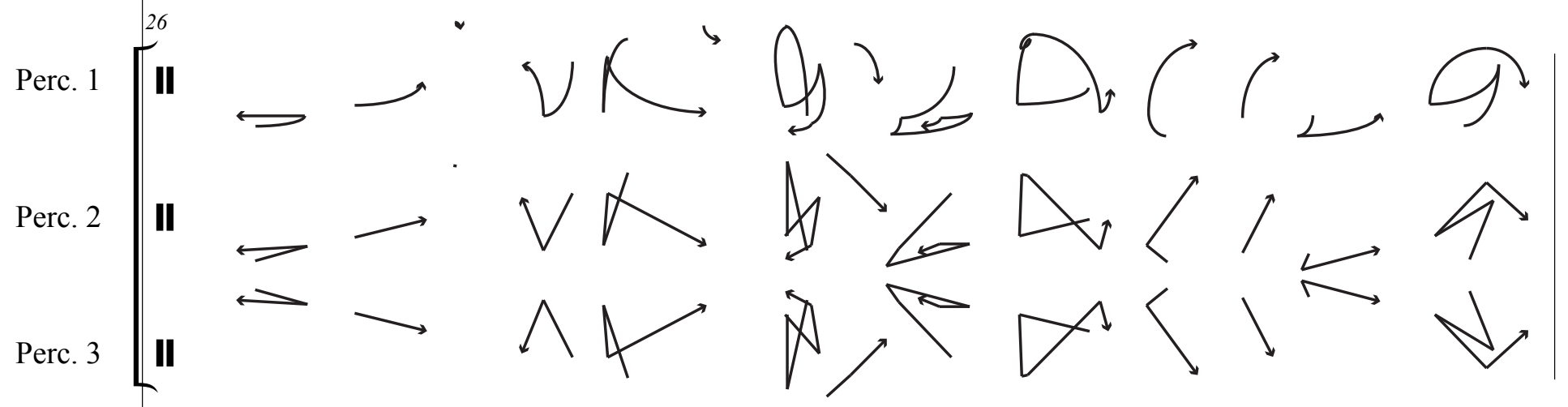

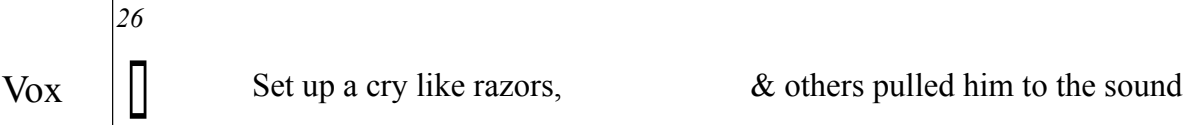

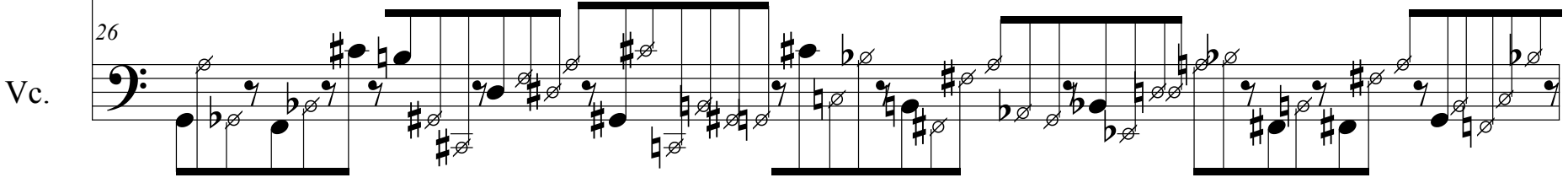


24

Fl.

B b Cl. 1

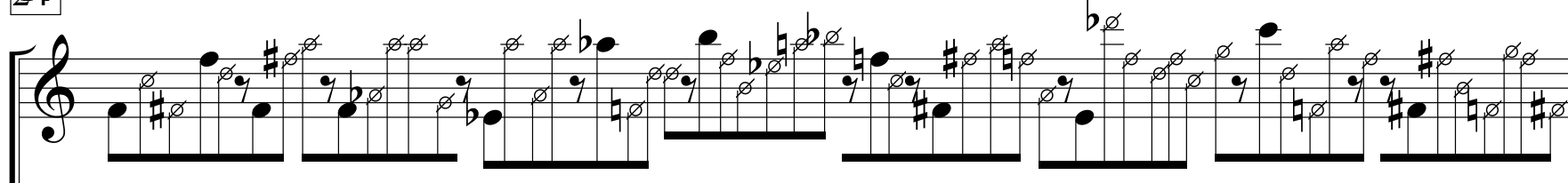

B b Cl. 2
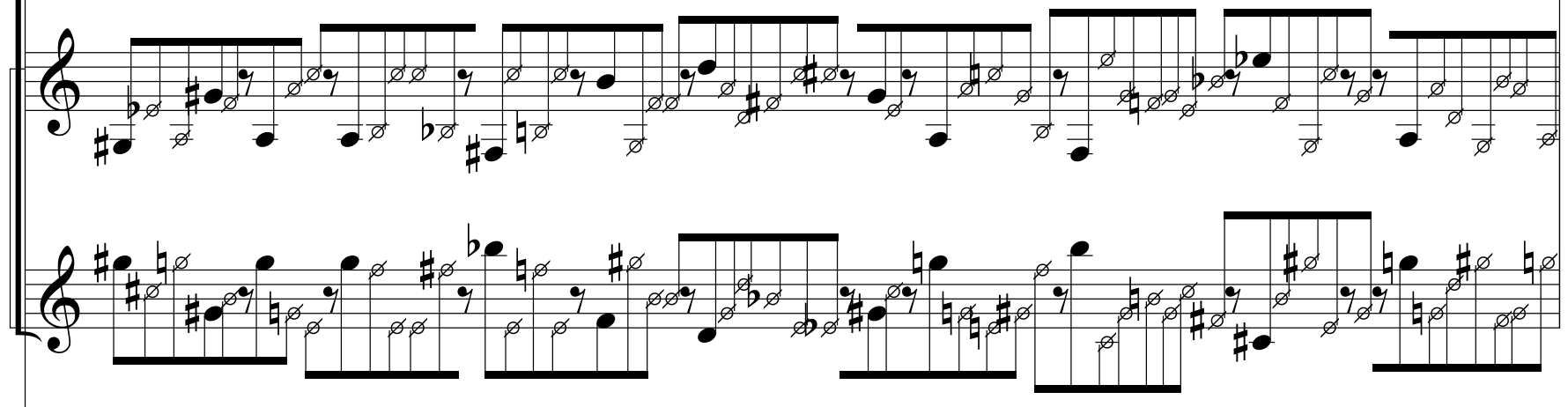

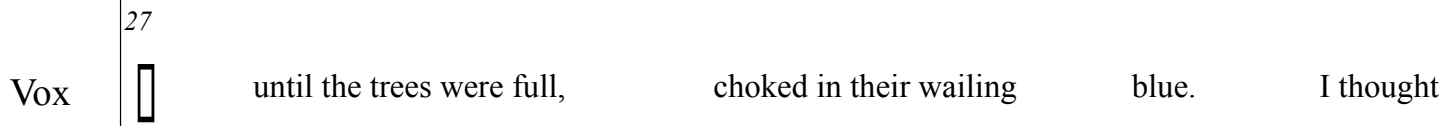

Fl.

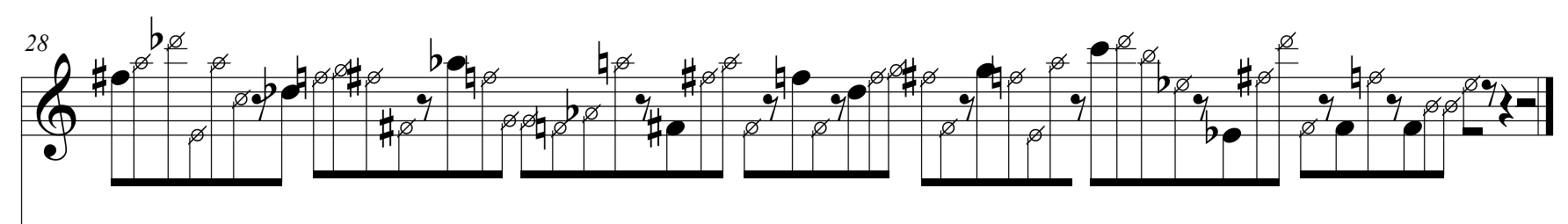

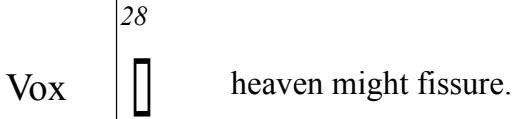

That it might give back what it took. 
Appendix 3: Three PFR-3 Poems by Jackson Mac Low 


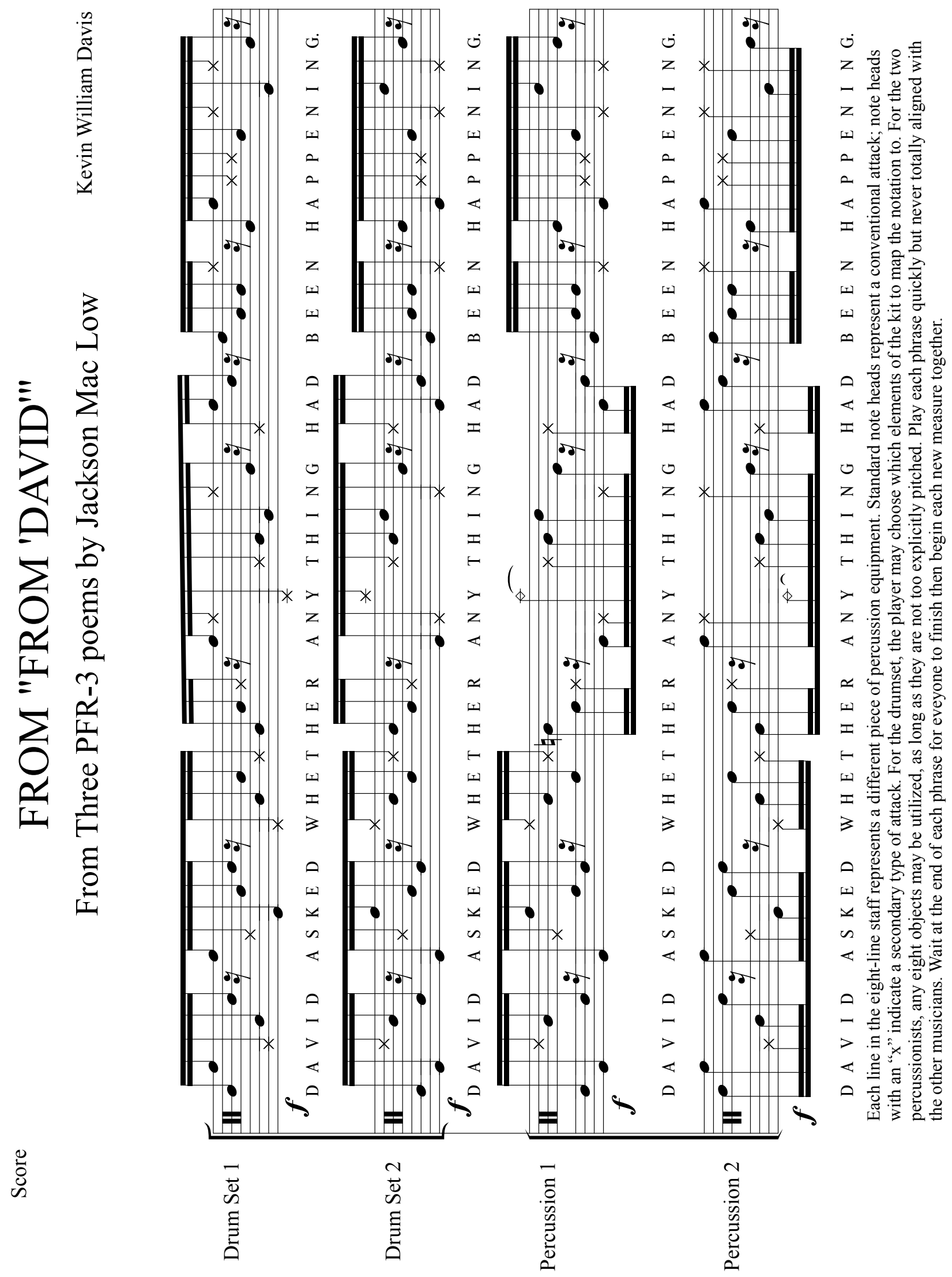




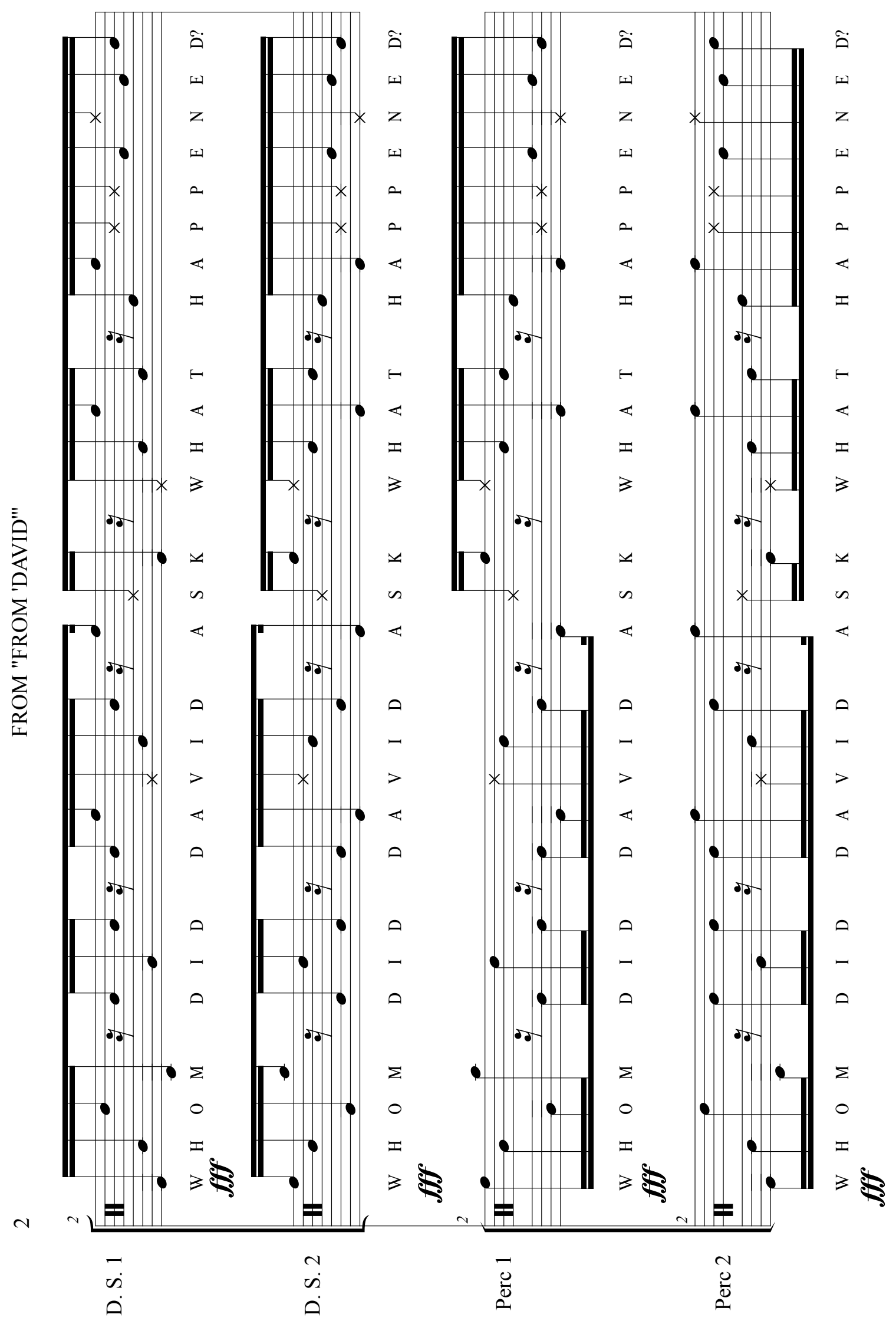




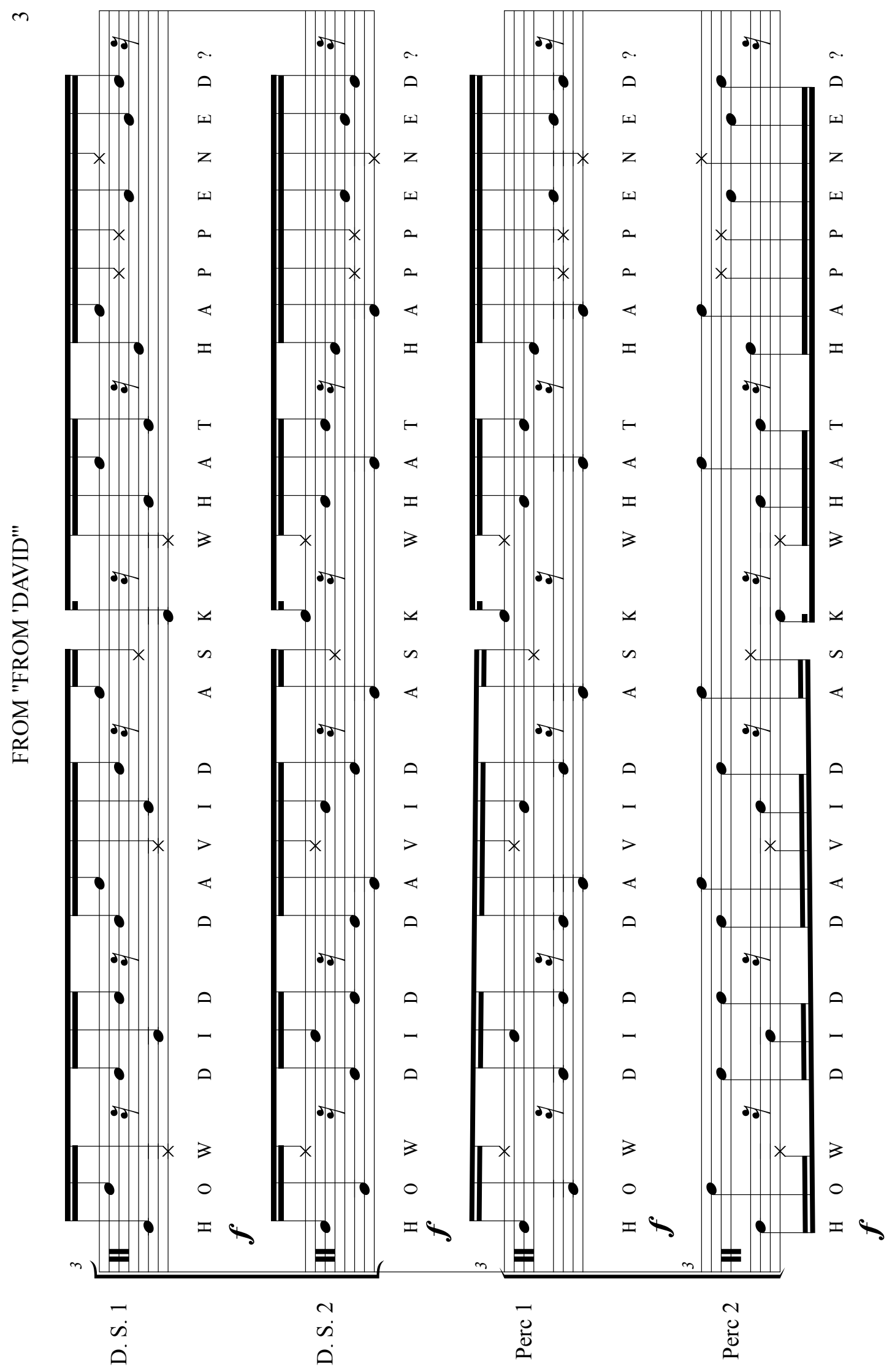




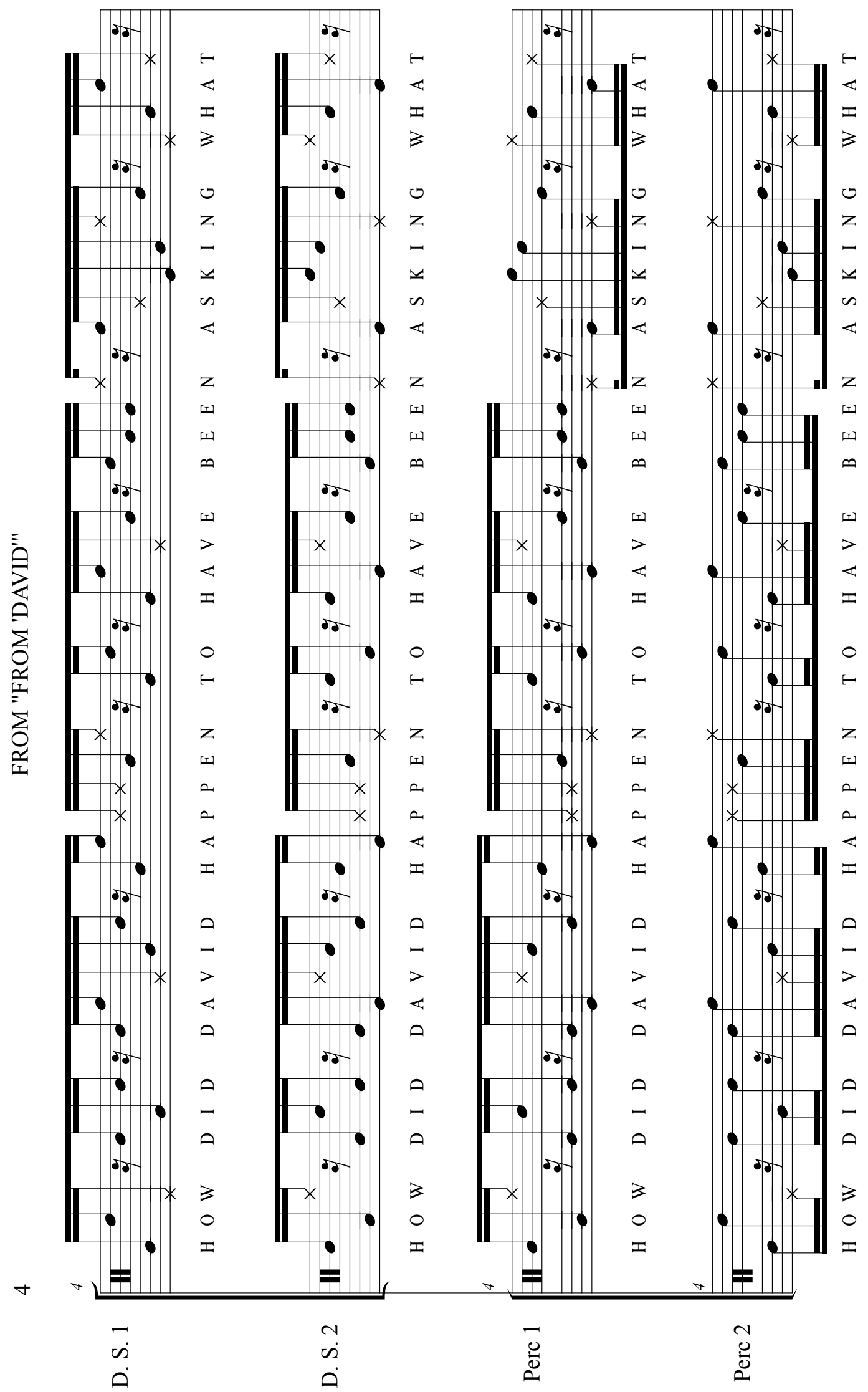




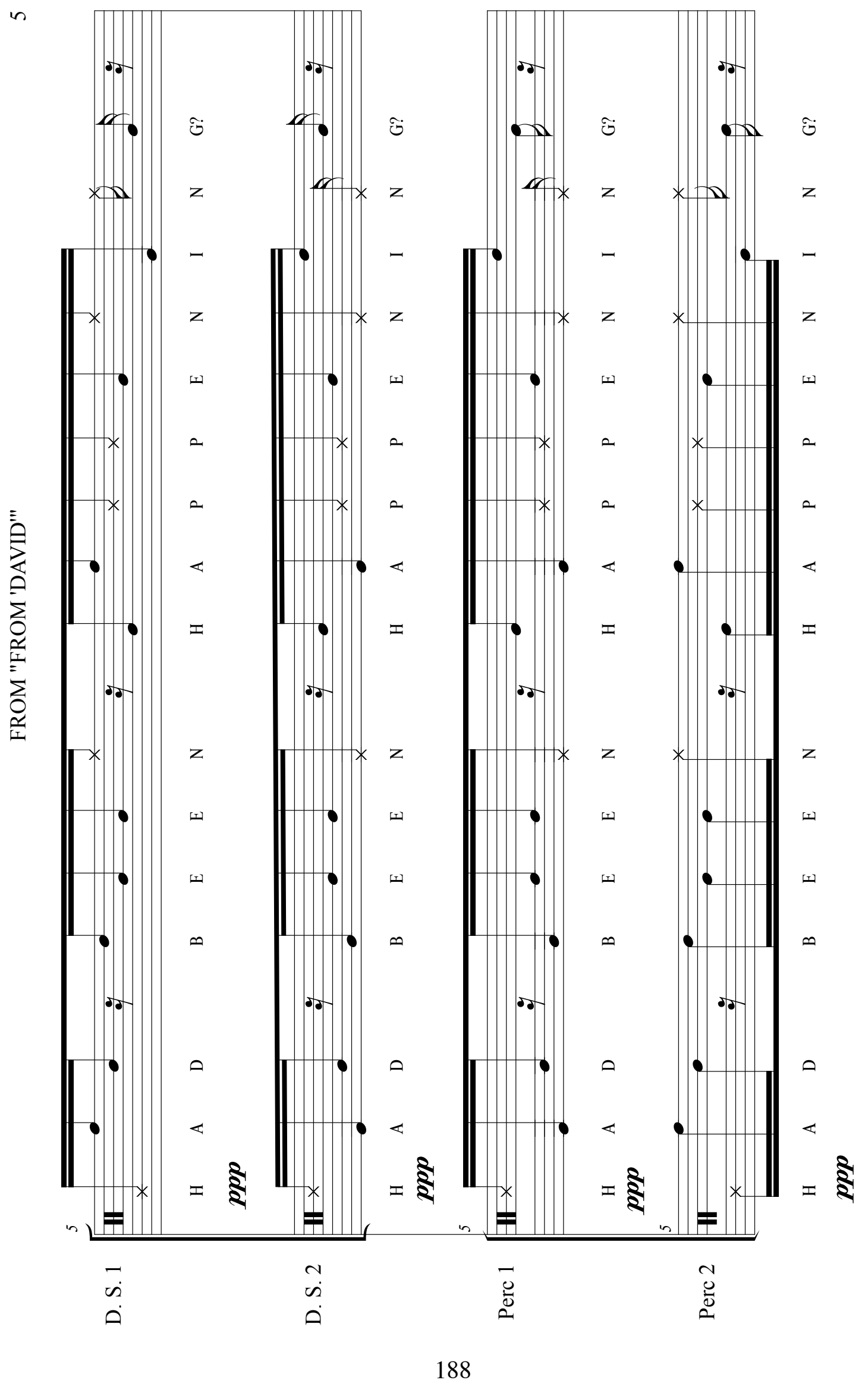




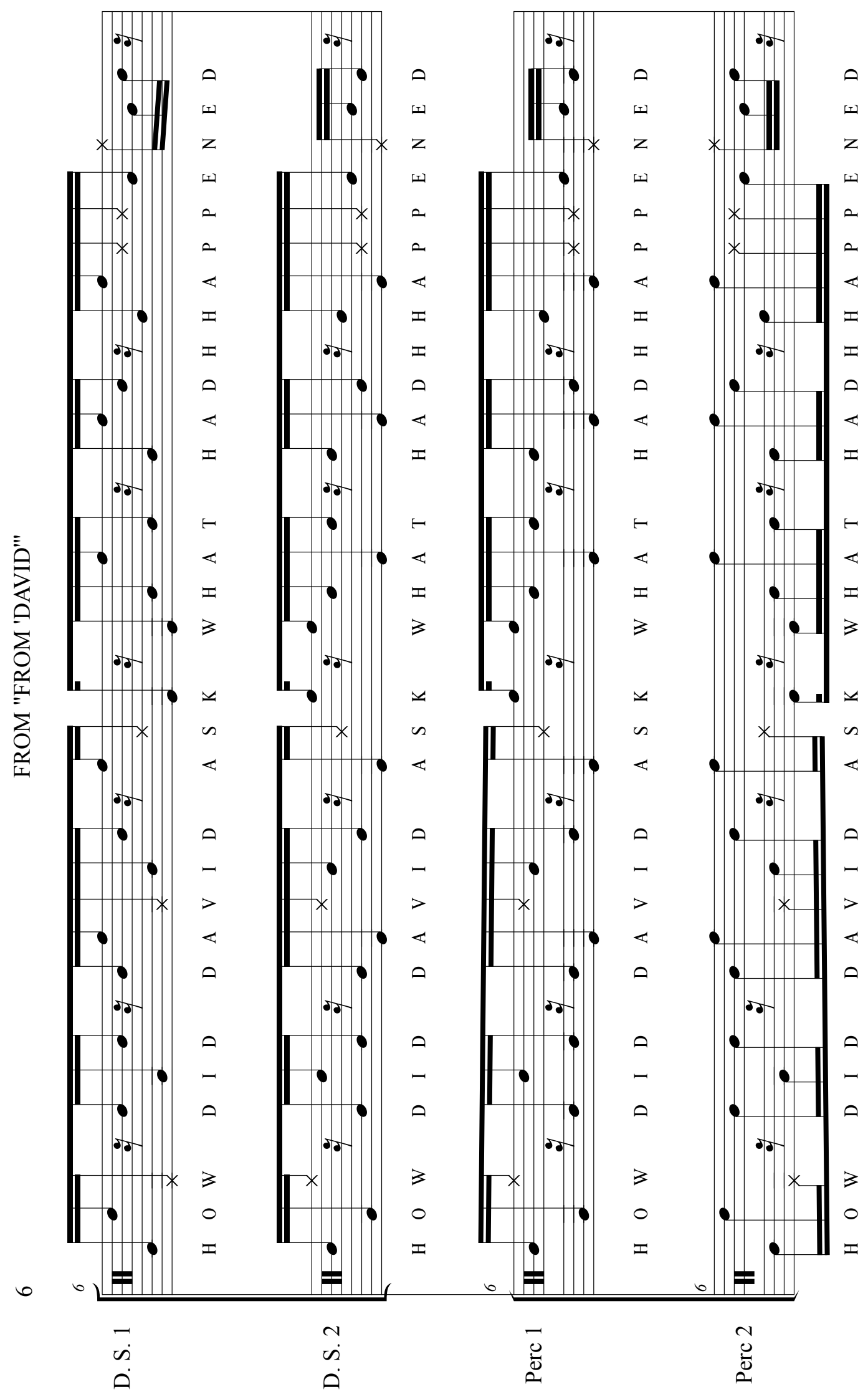




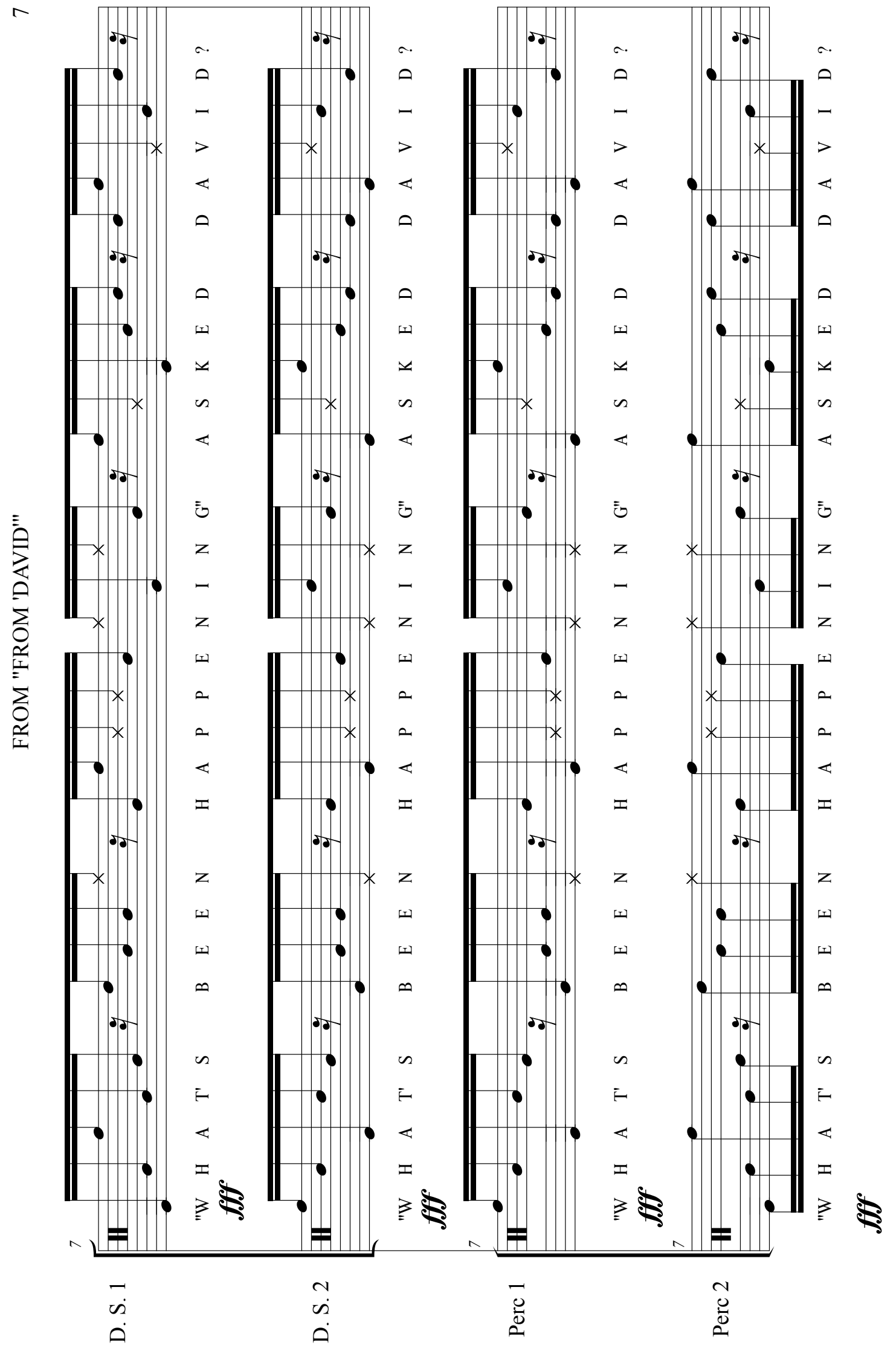




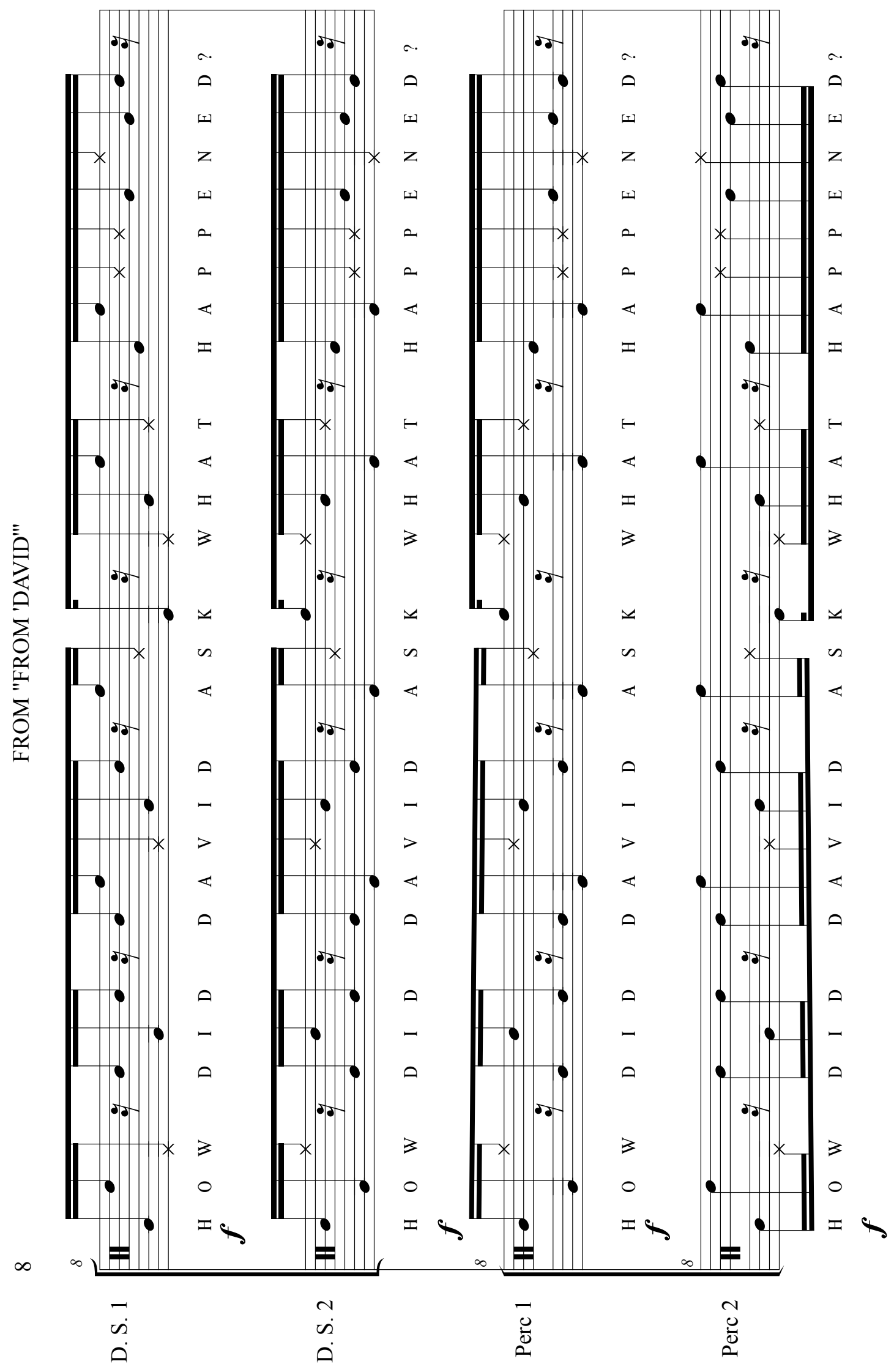




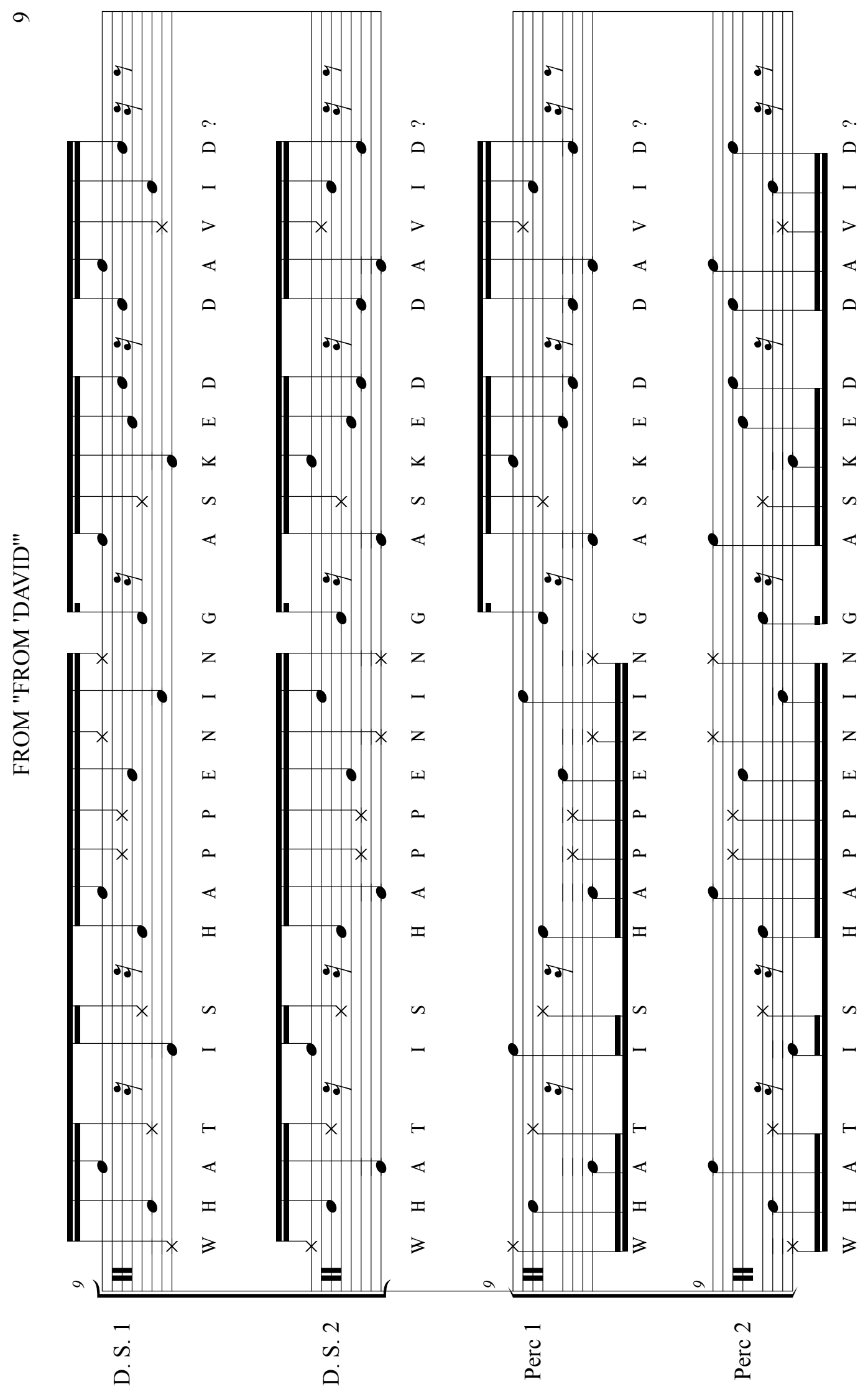




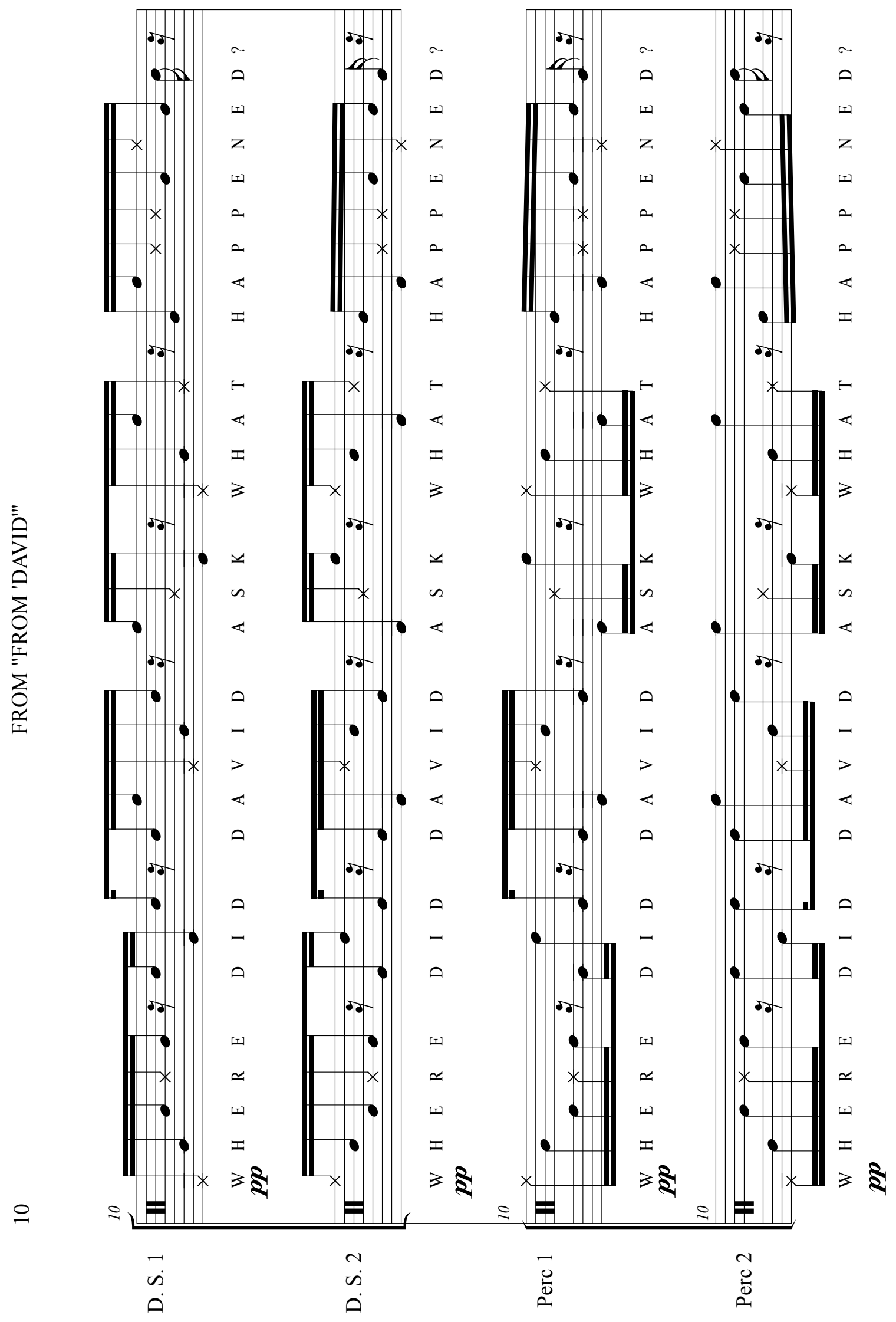




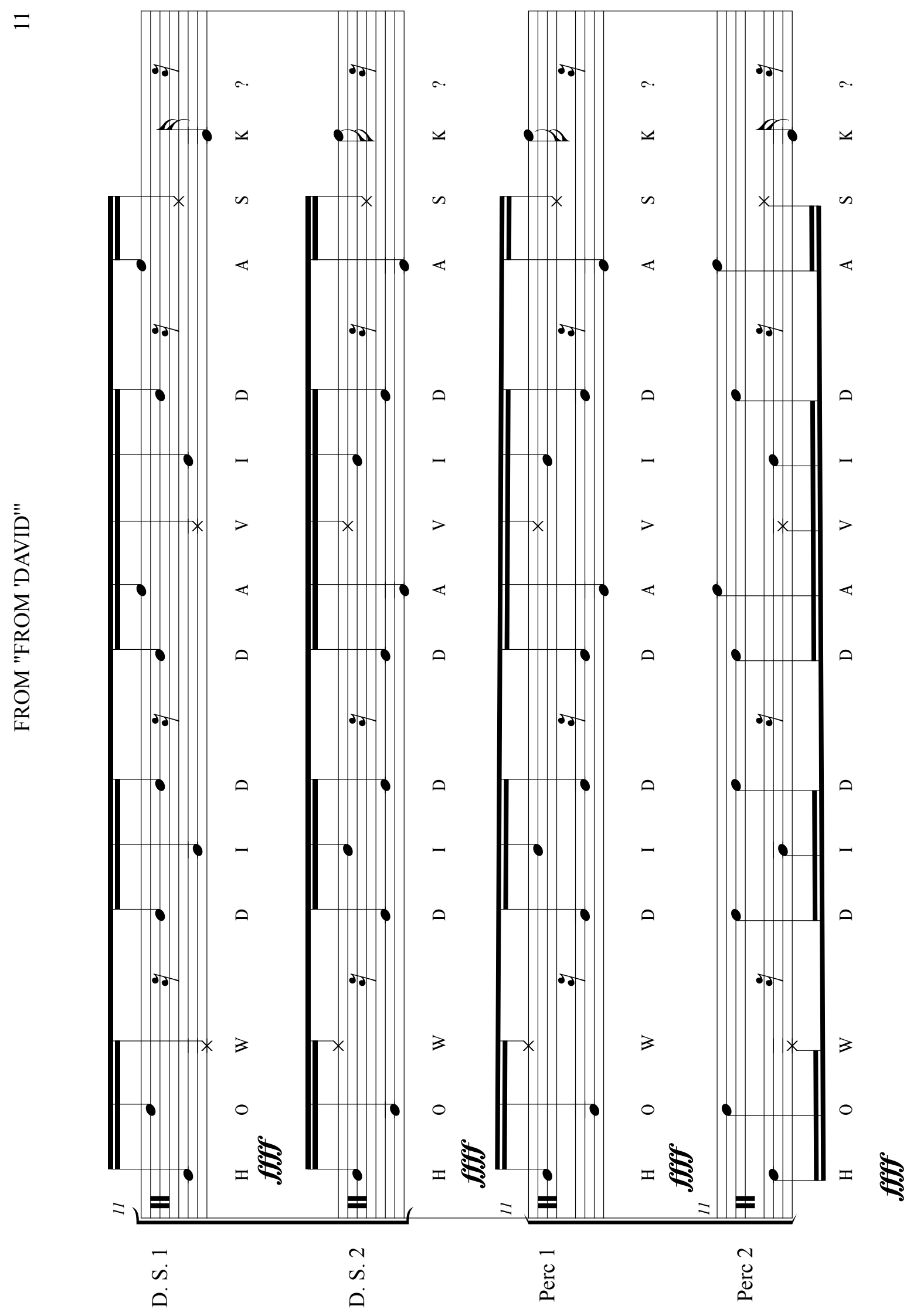




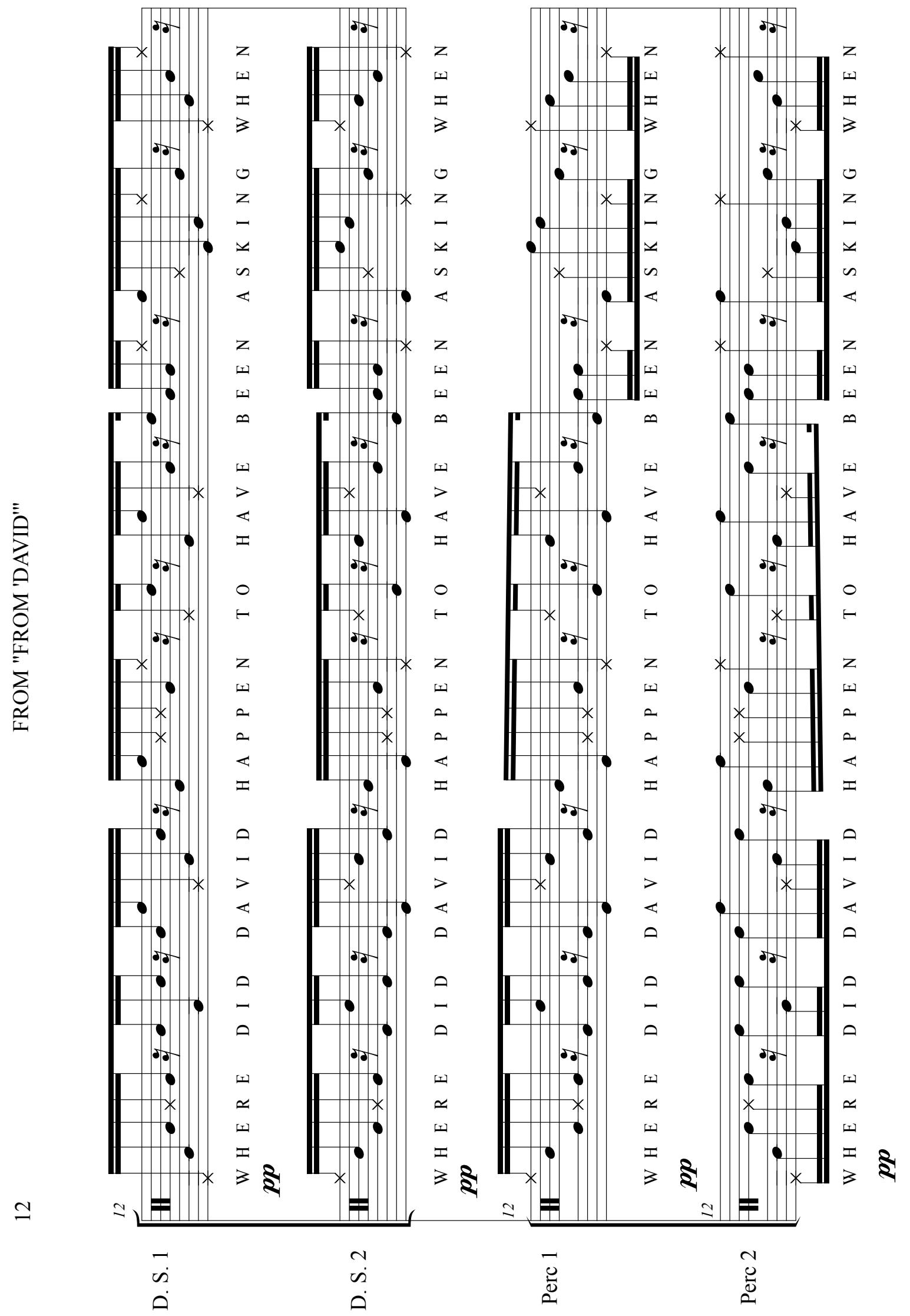




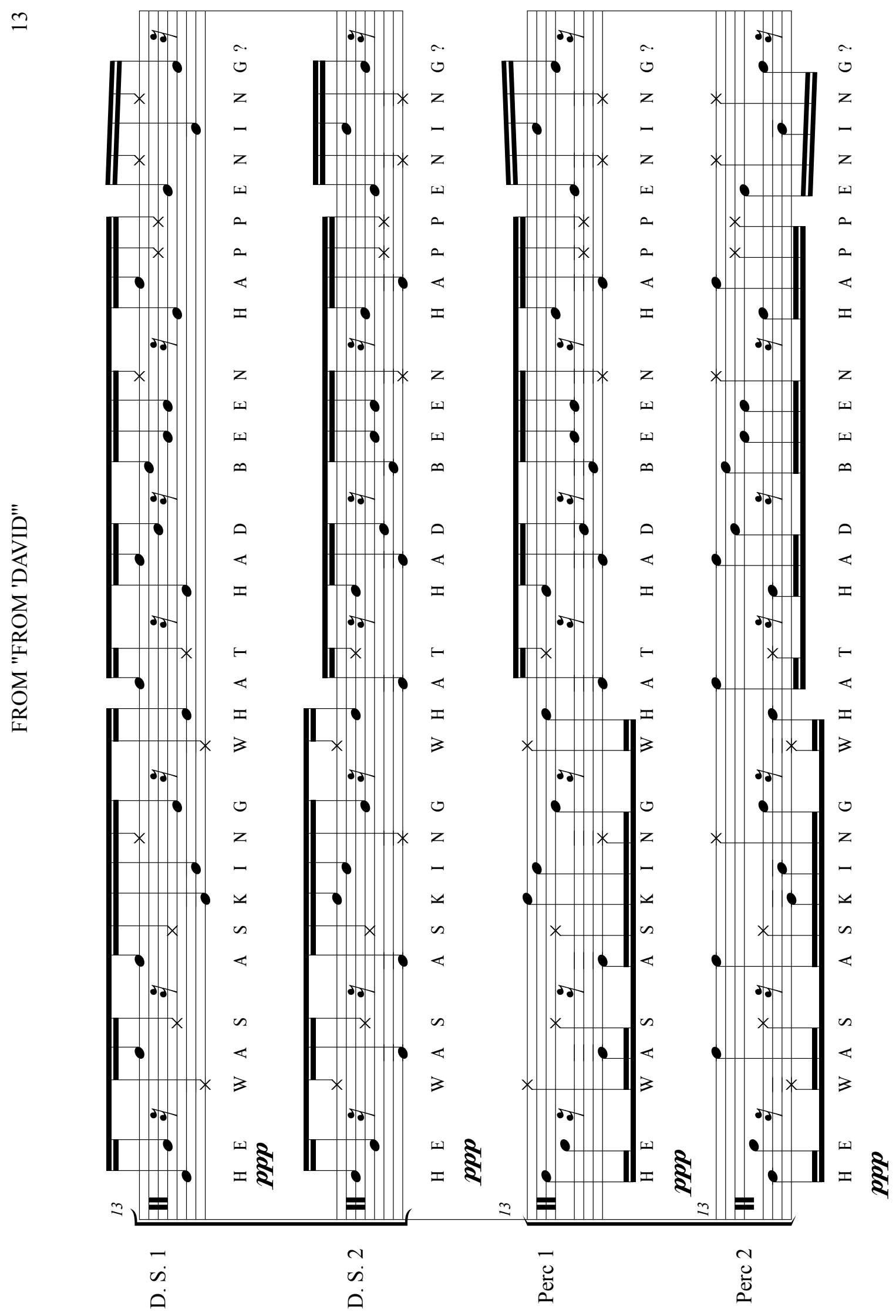




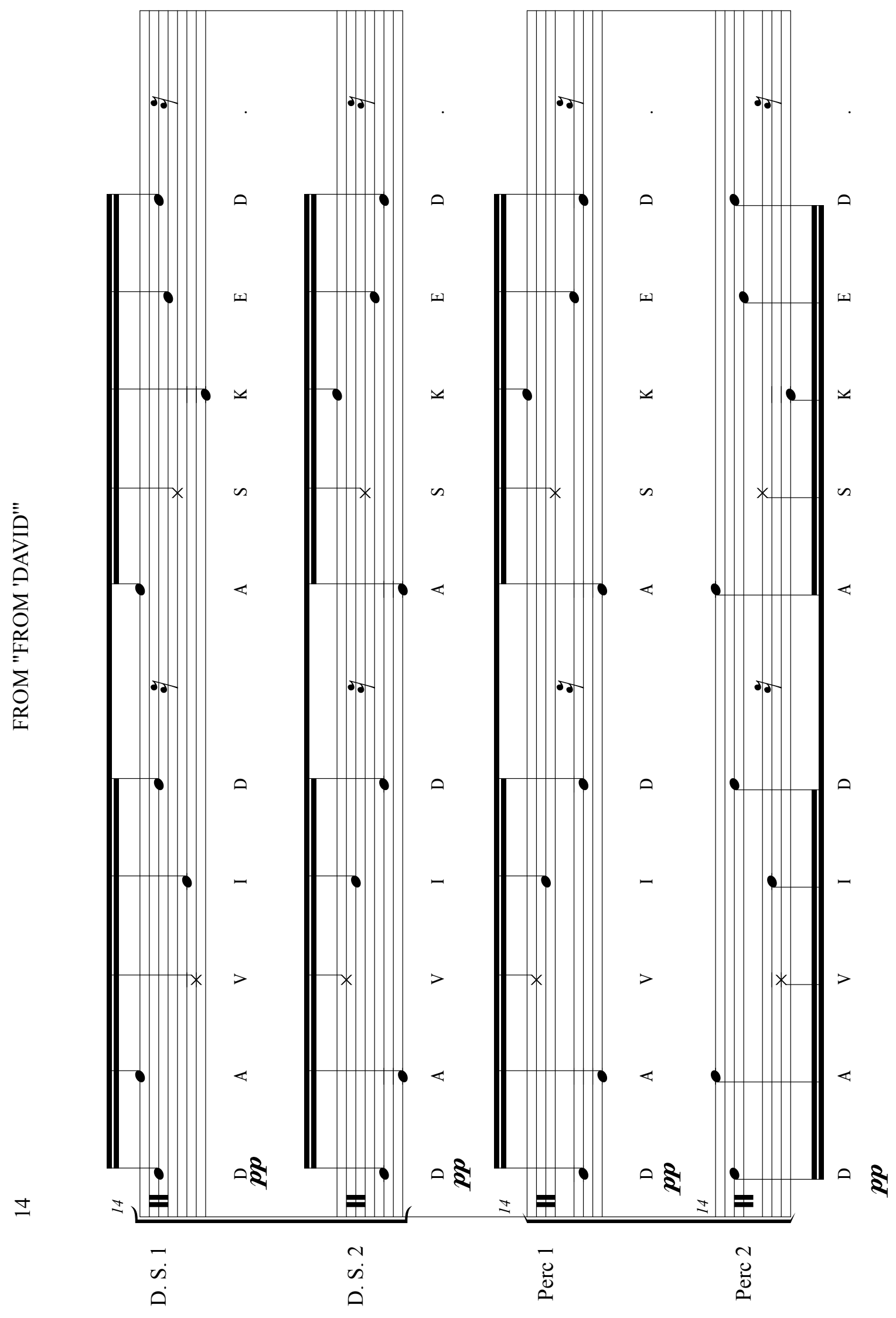




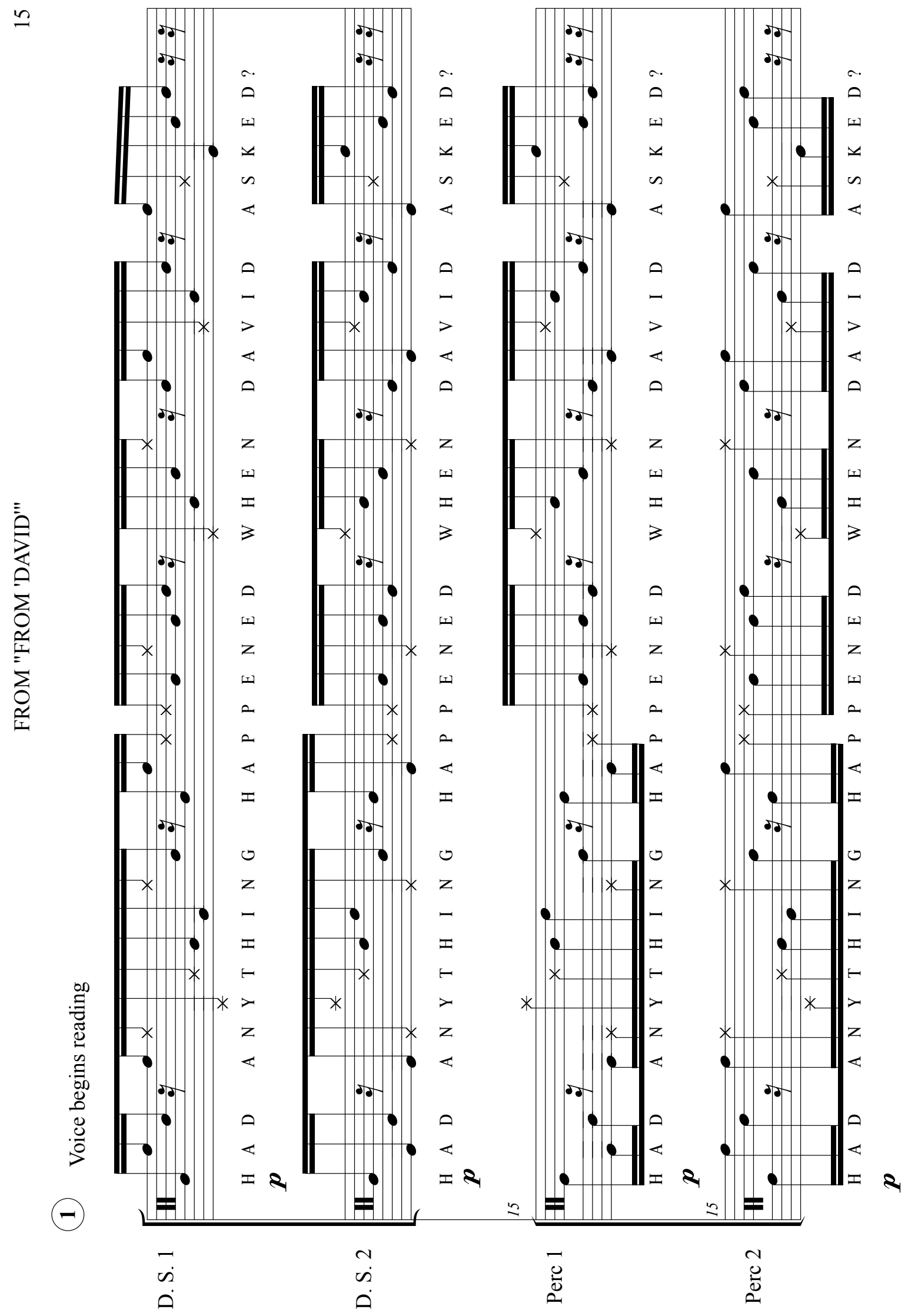




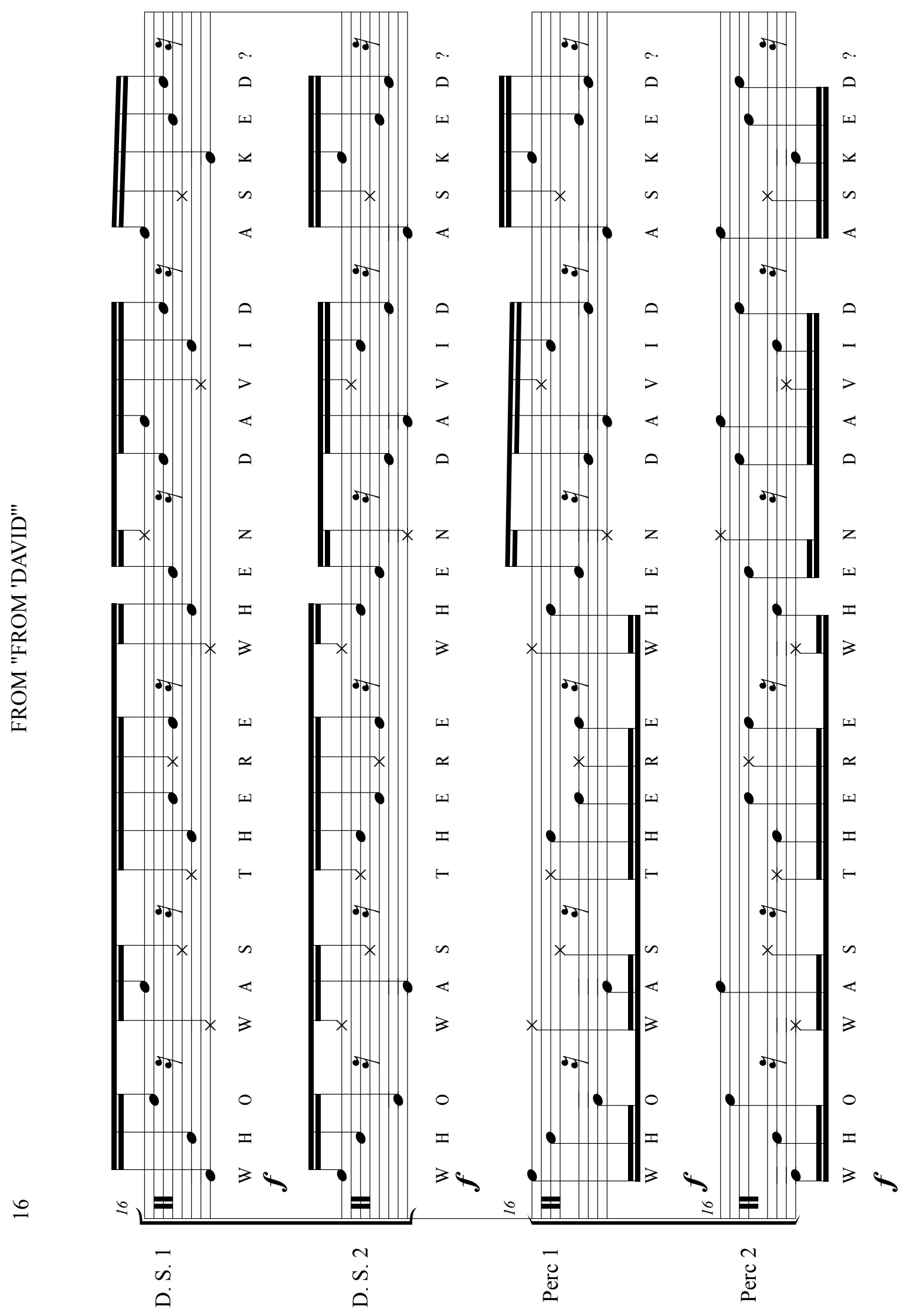




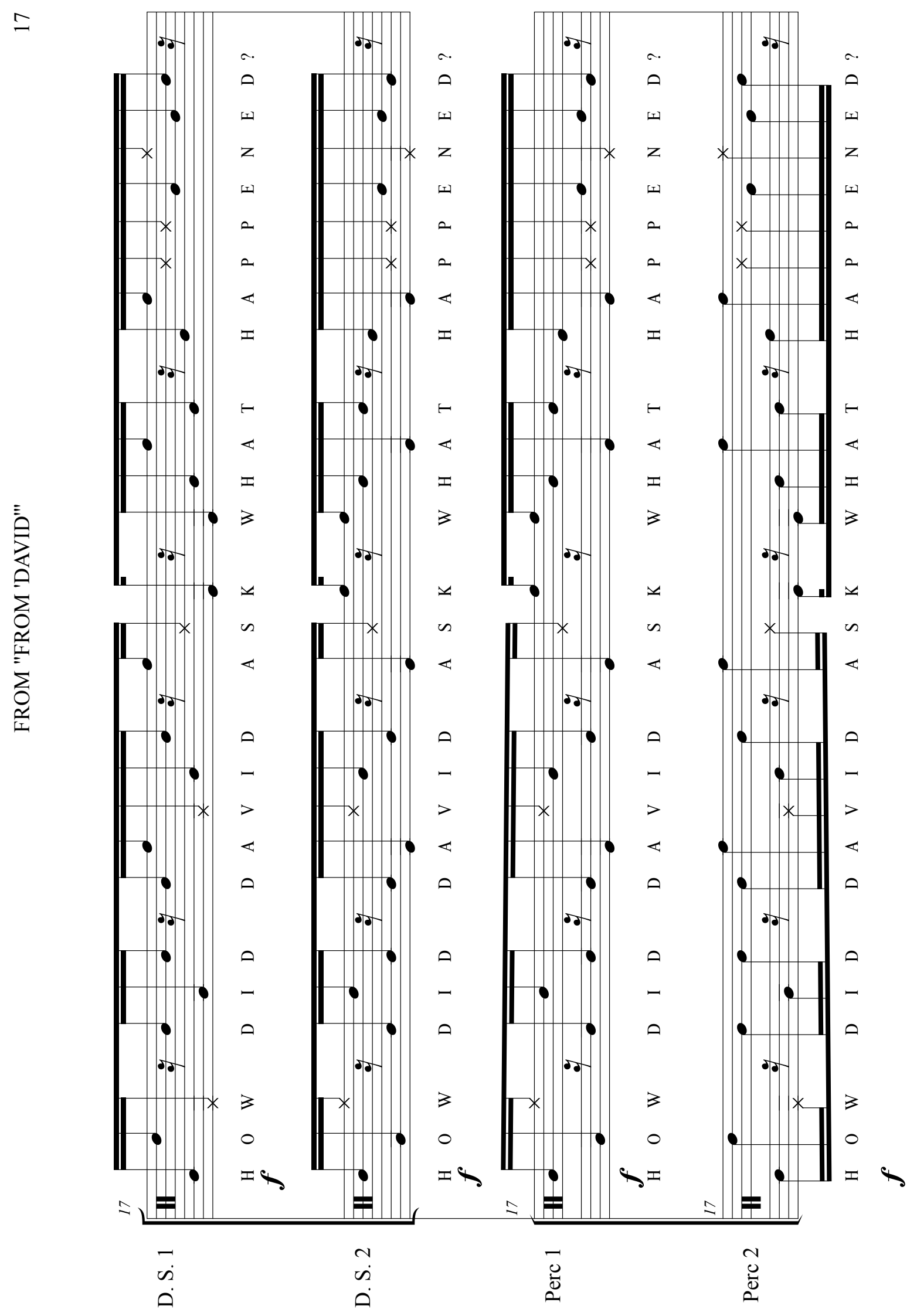




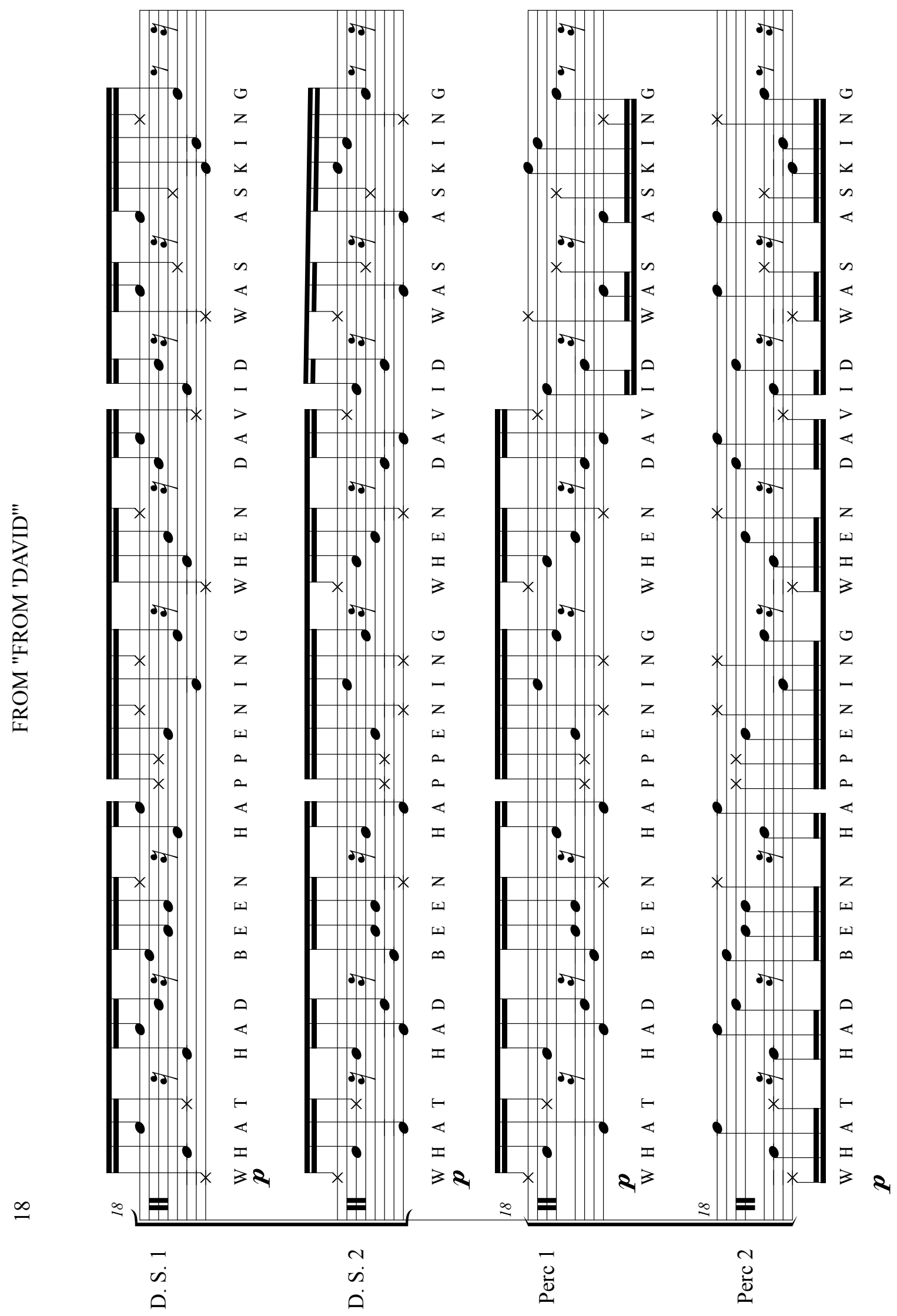




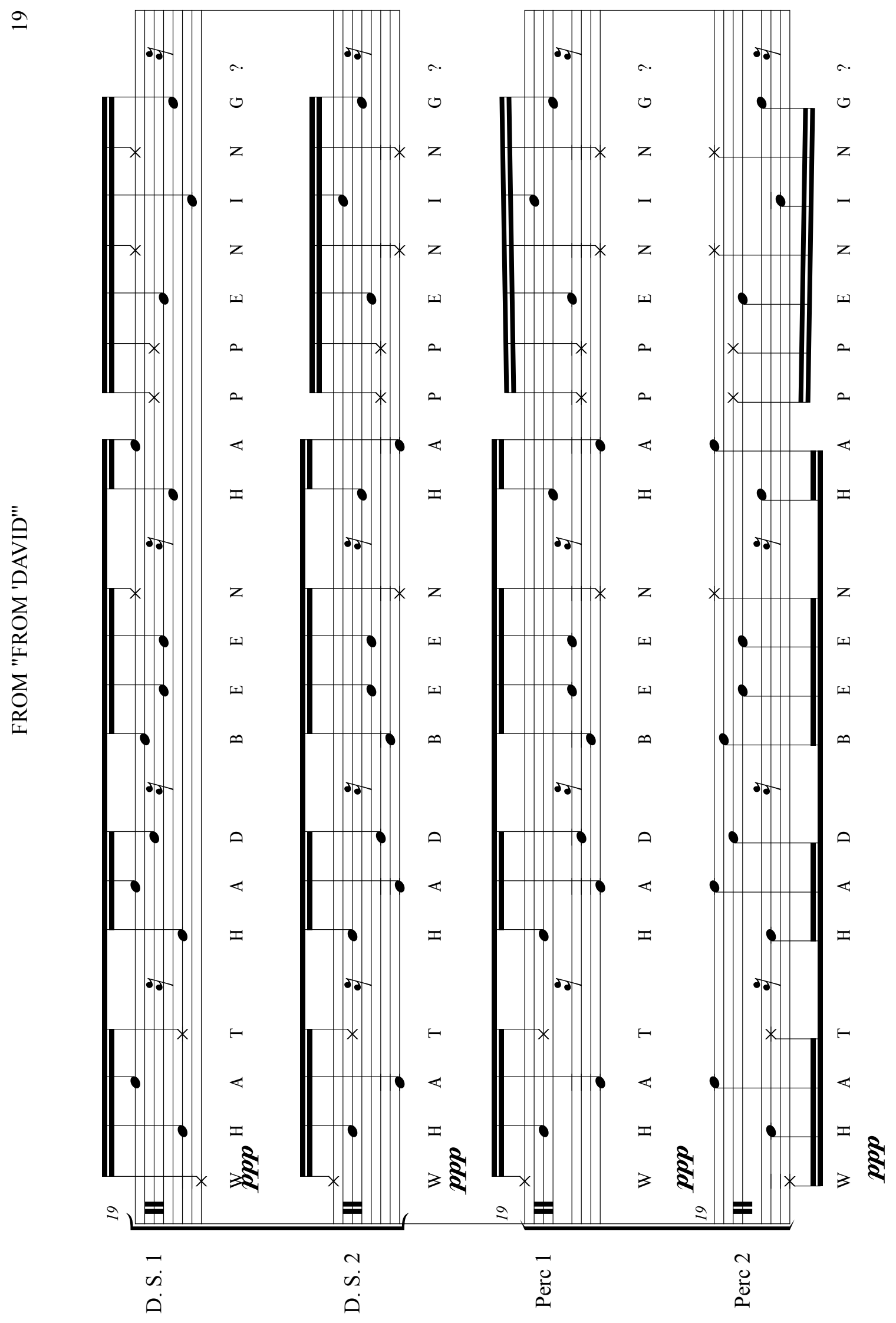




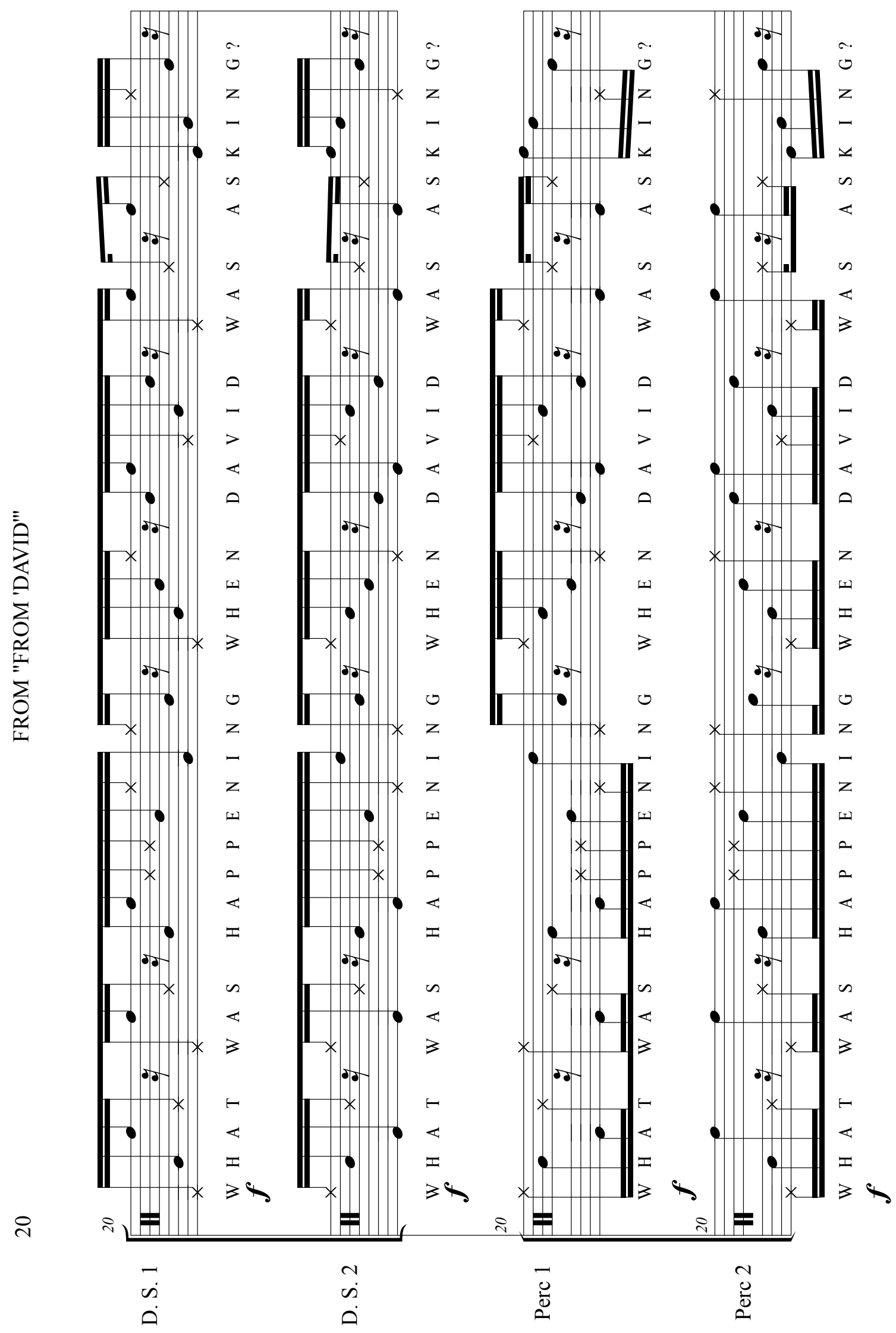




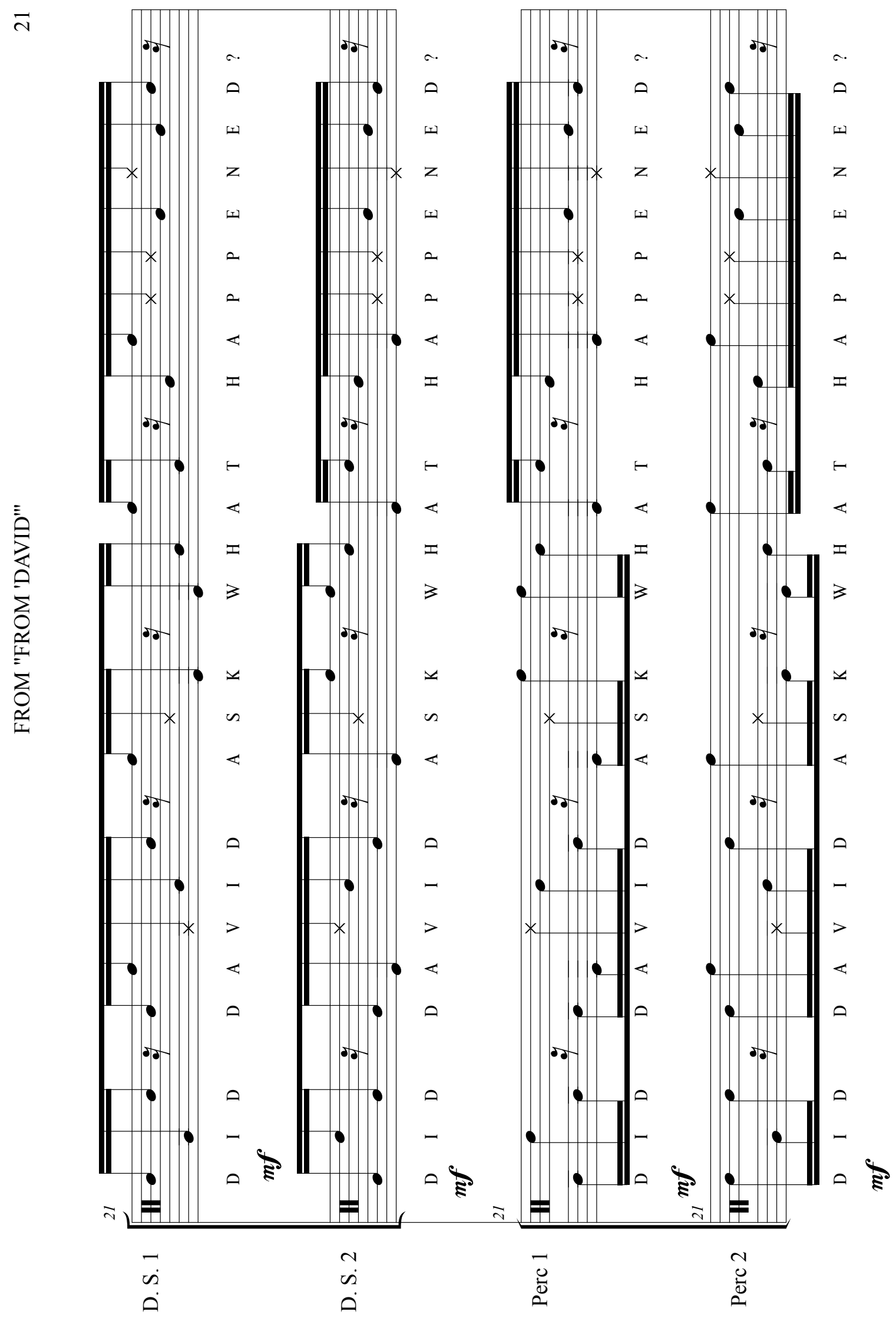




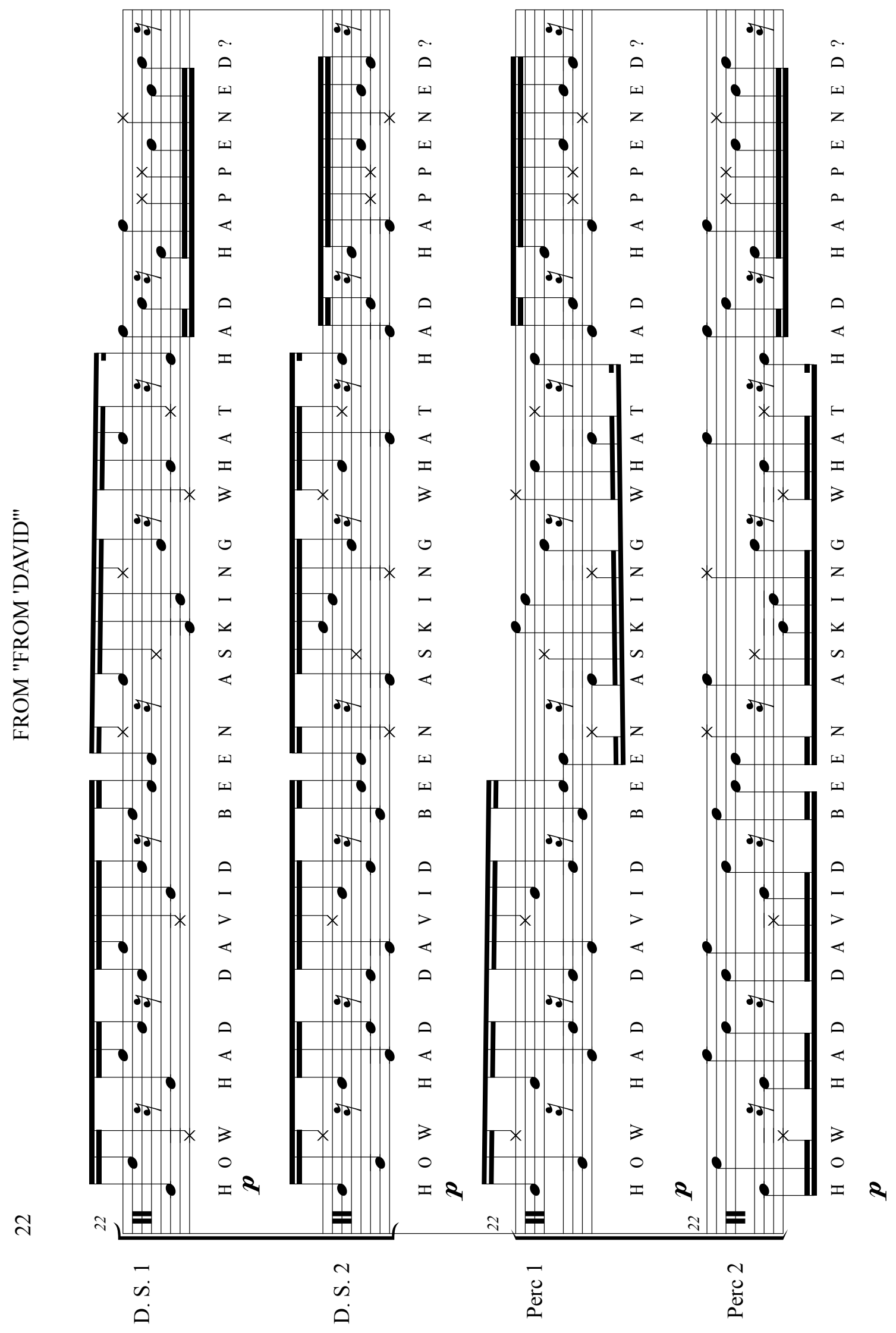




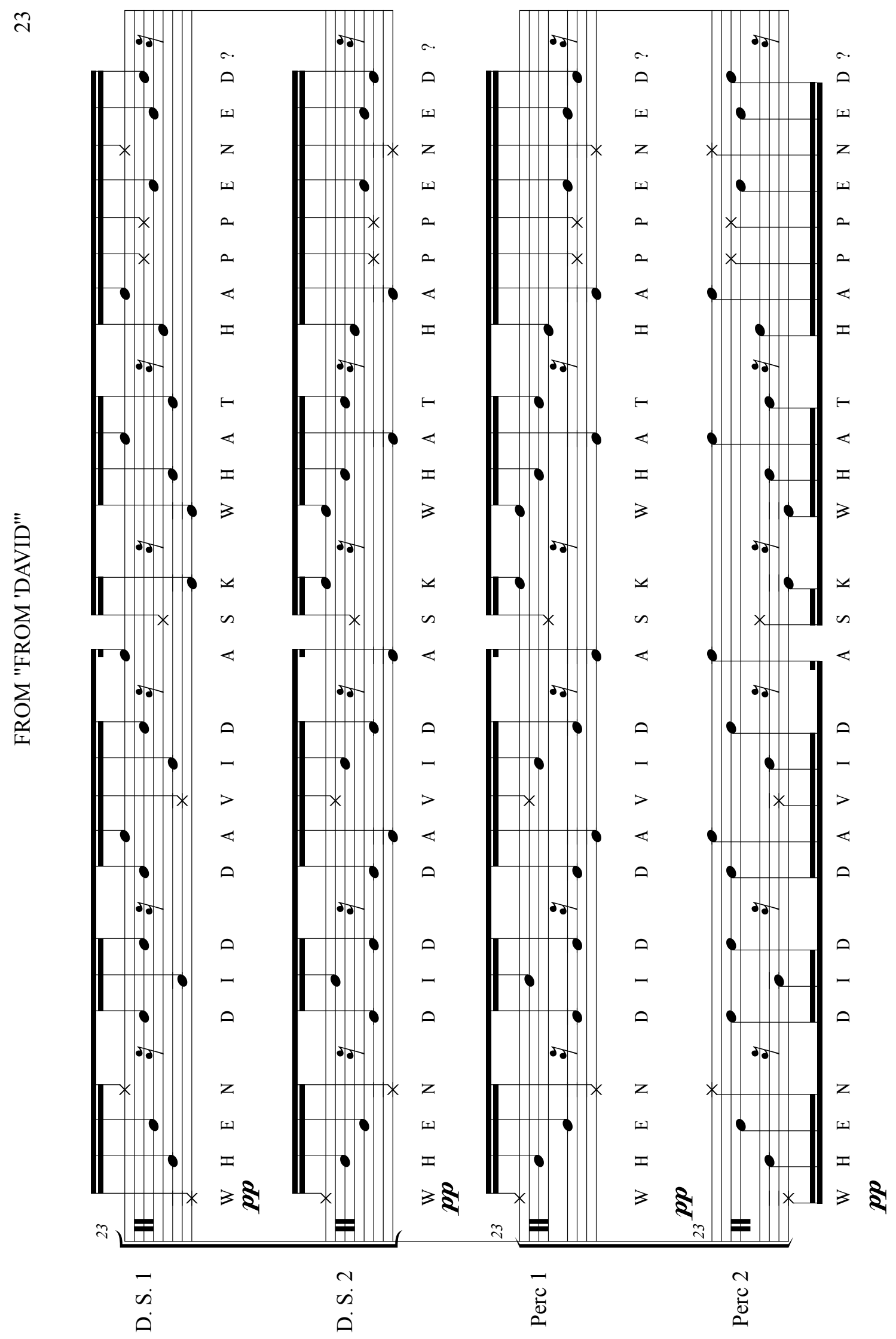




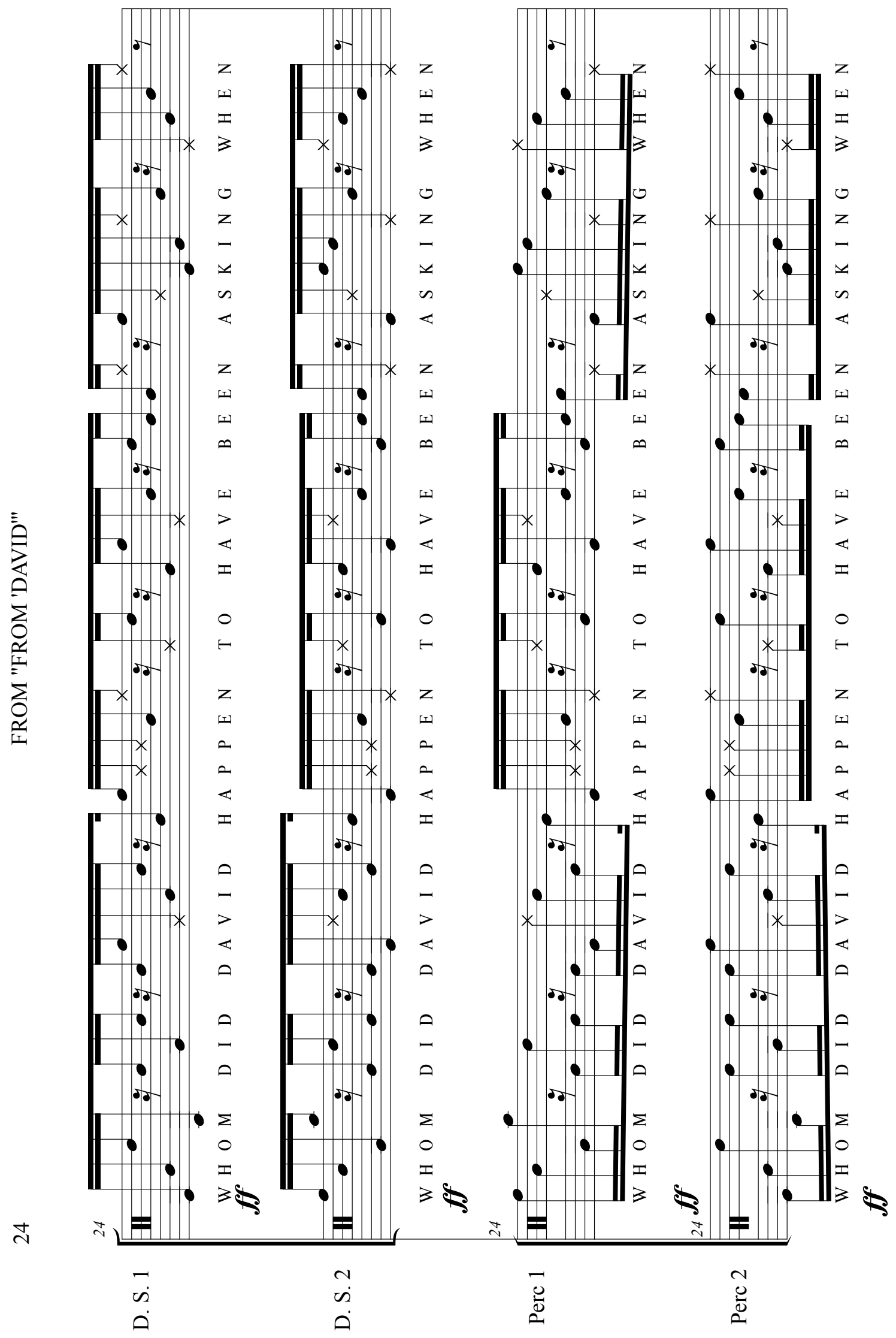




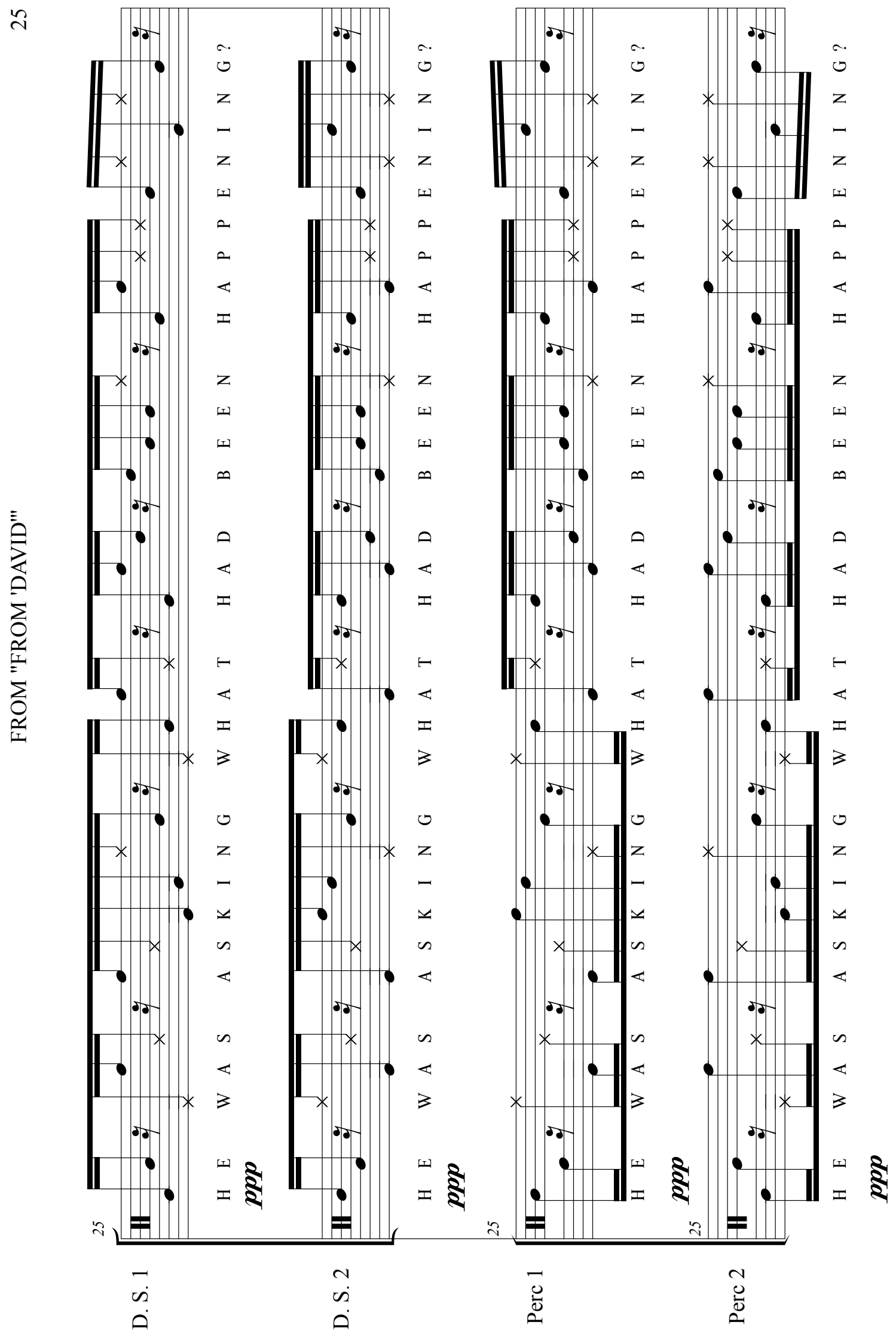




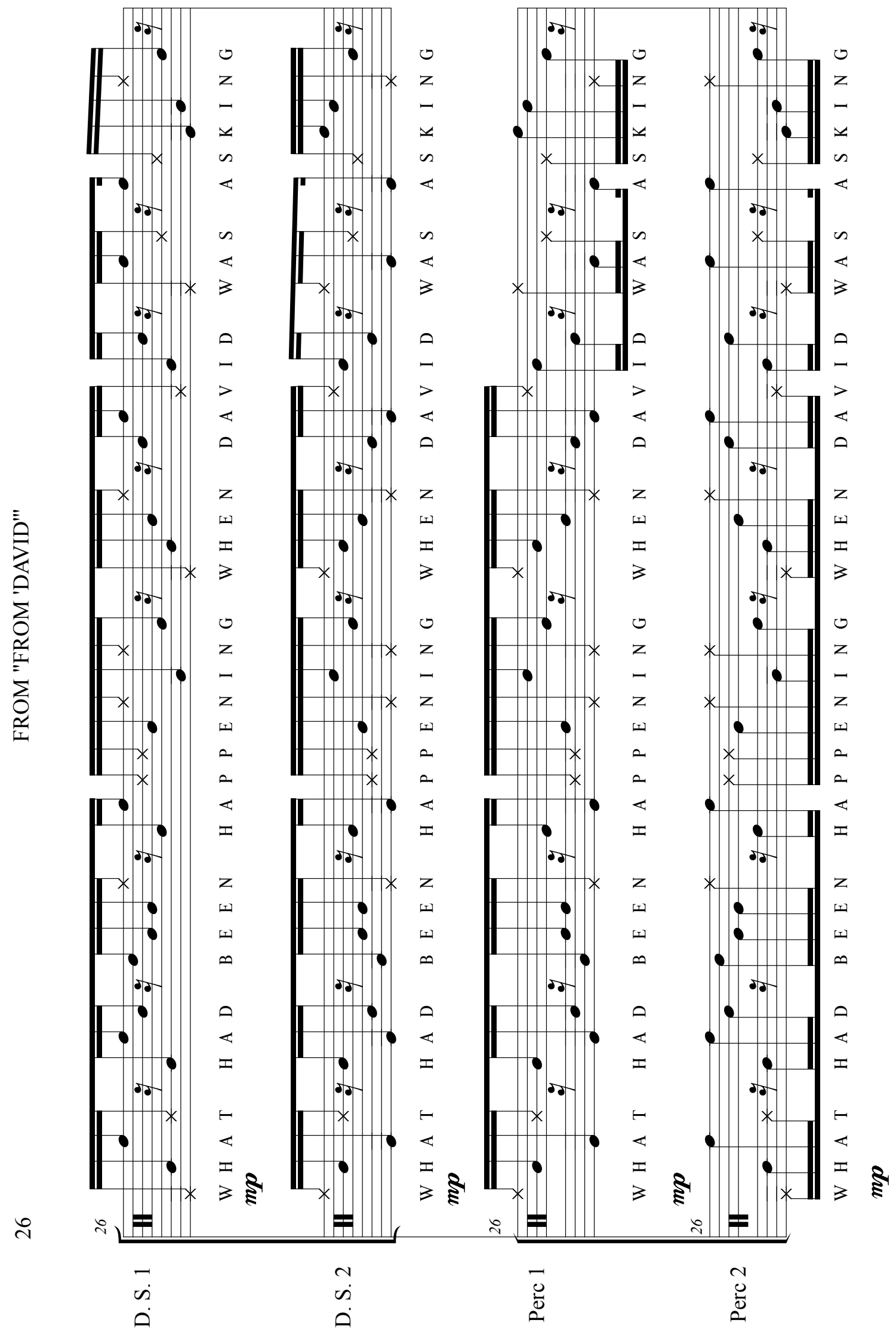




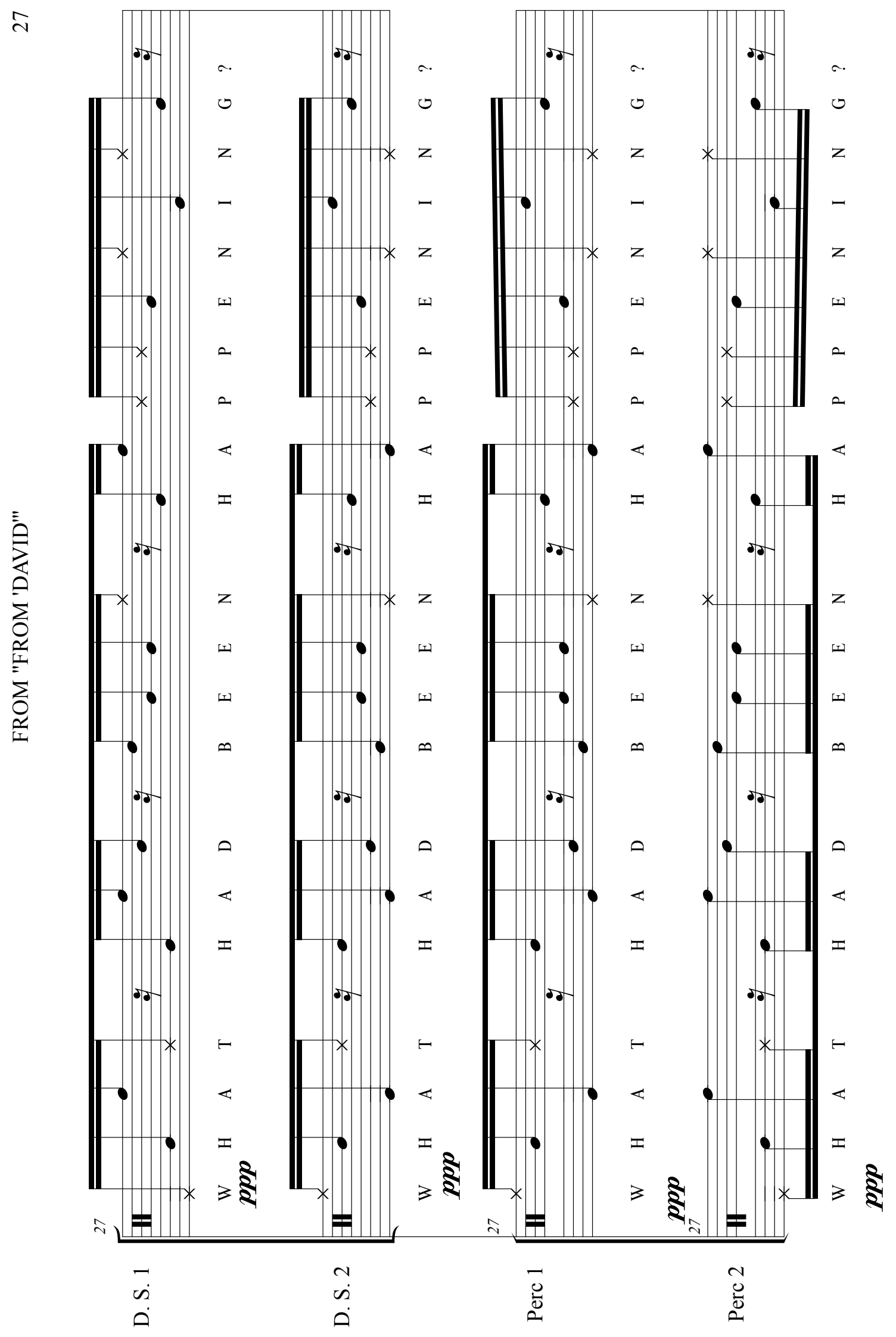




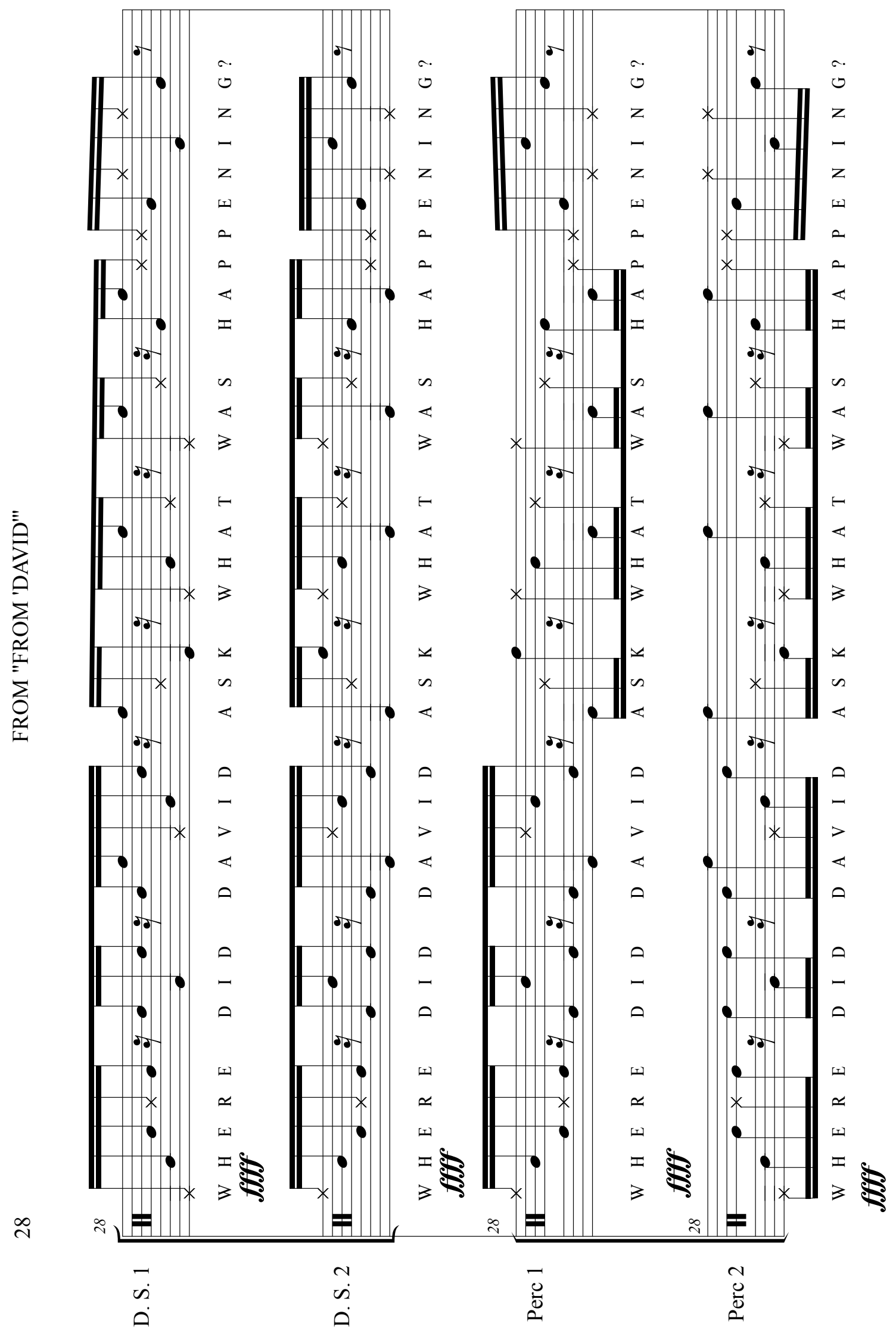




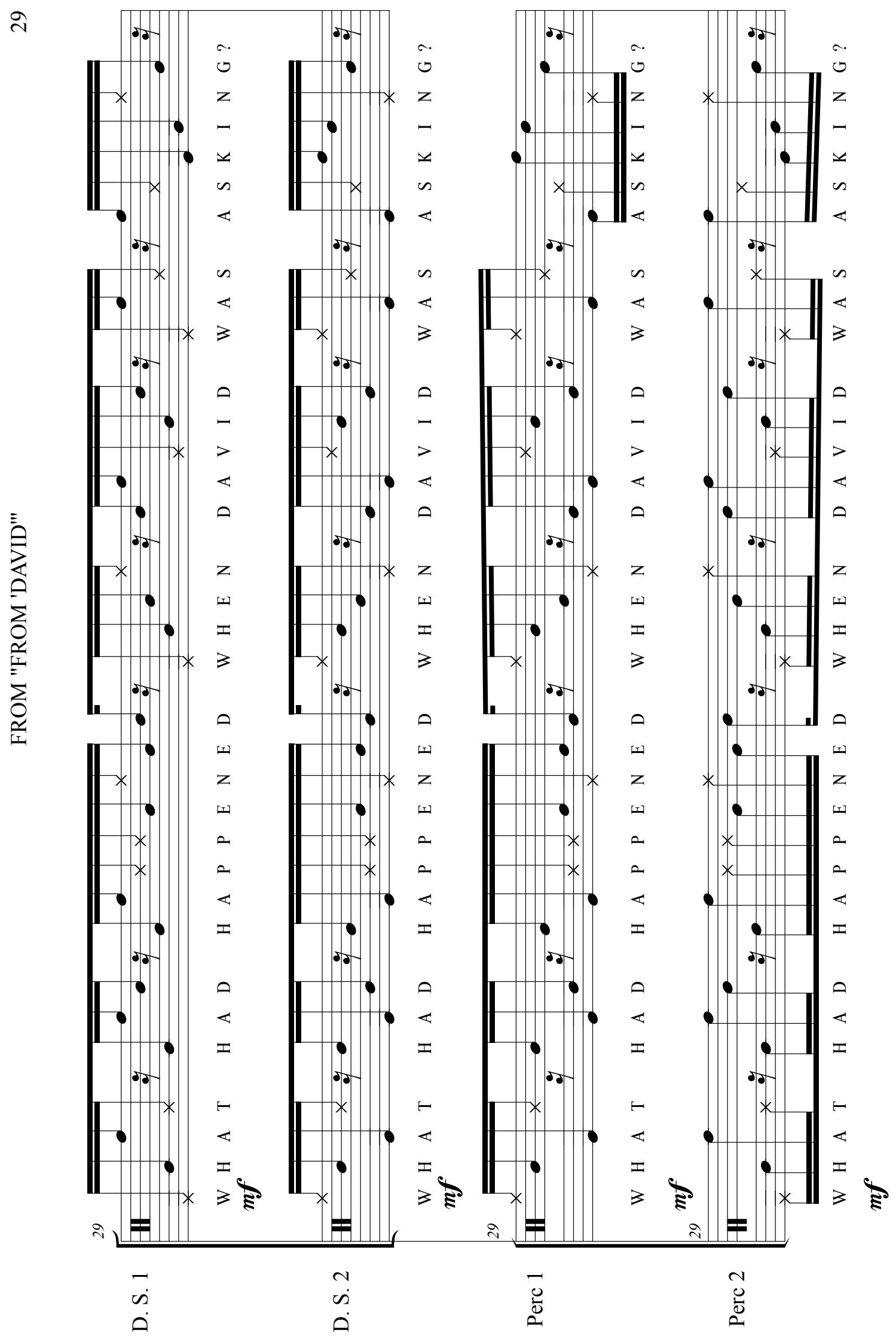




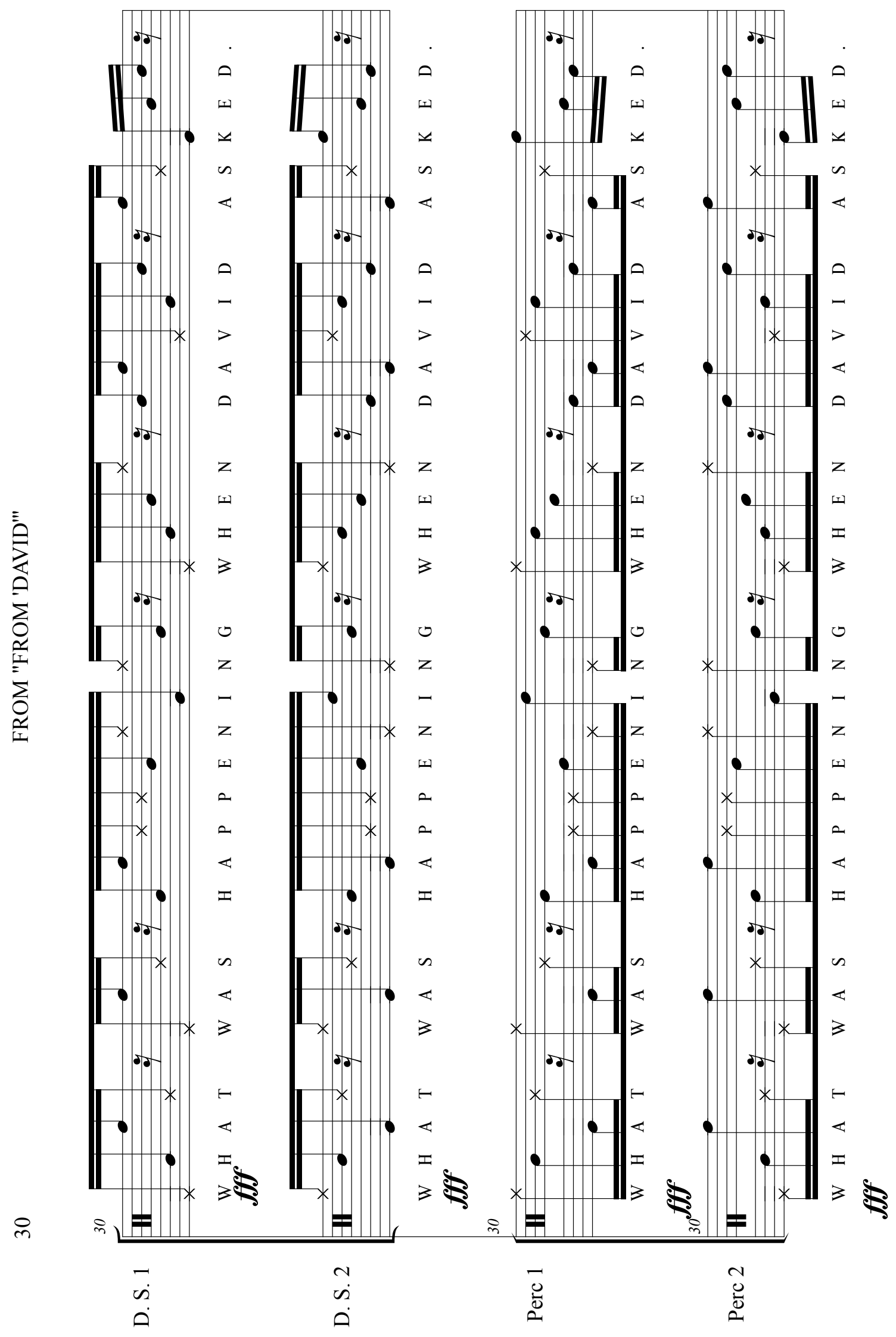




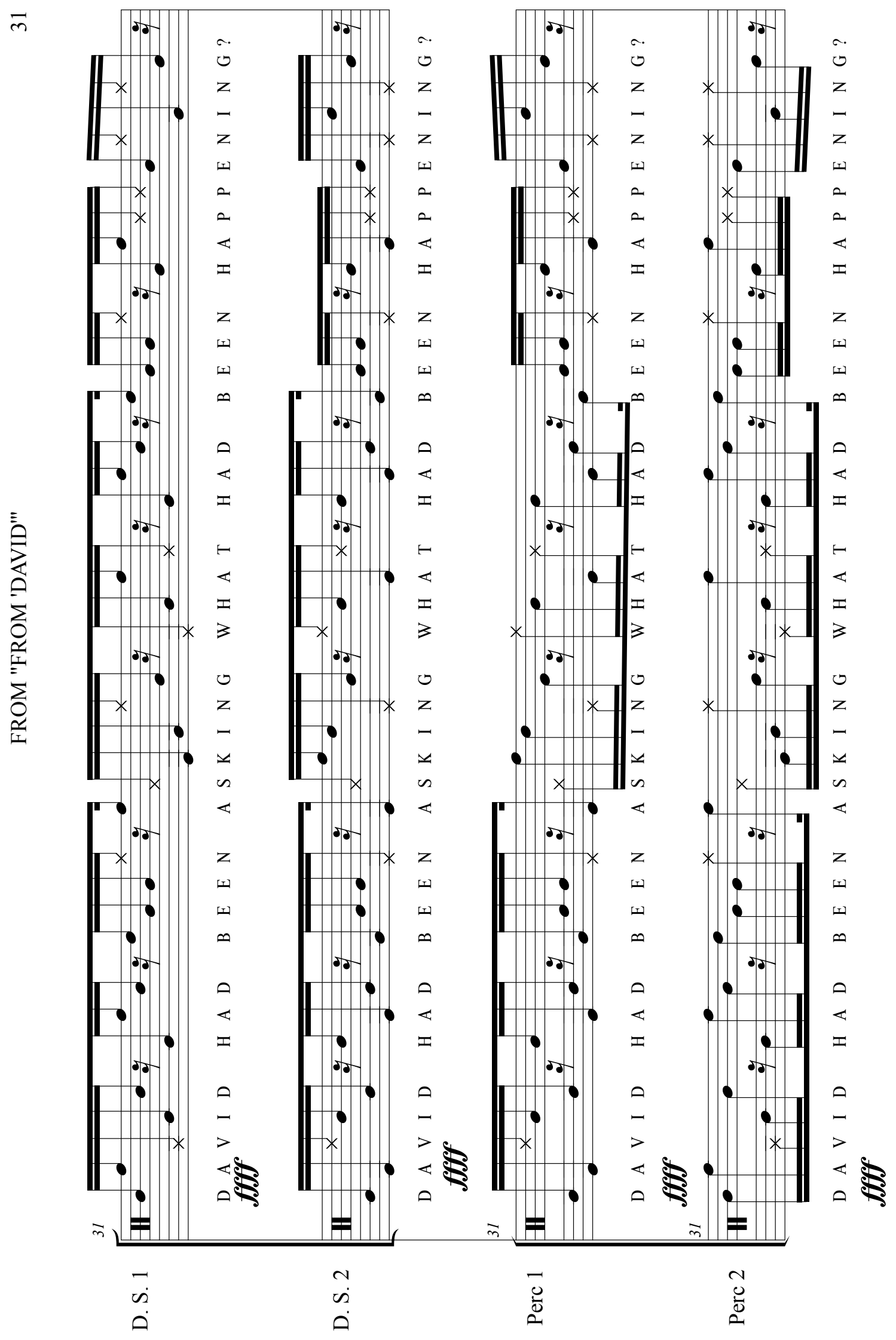




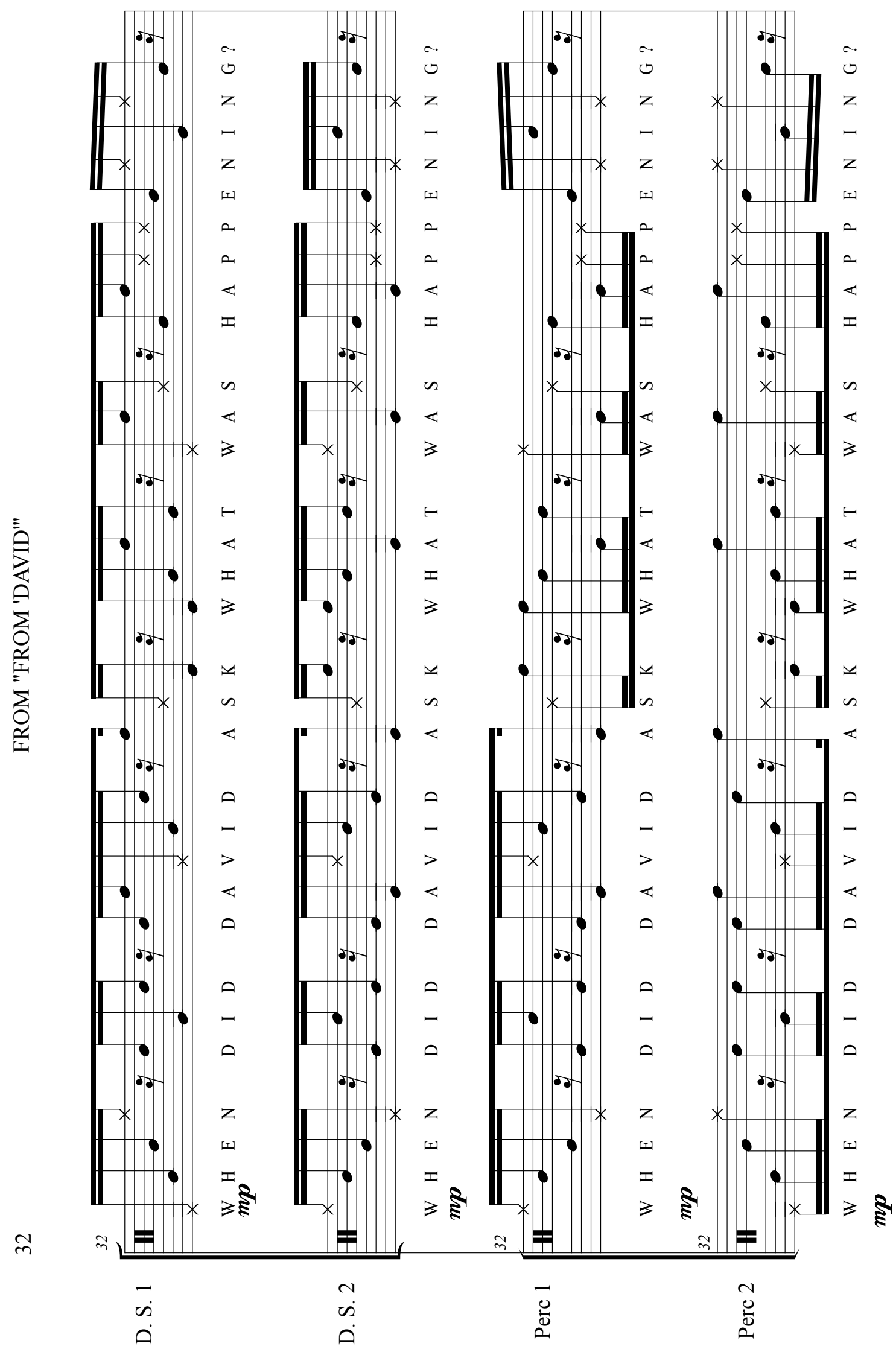




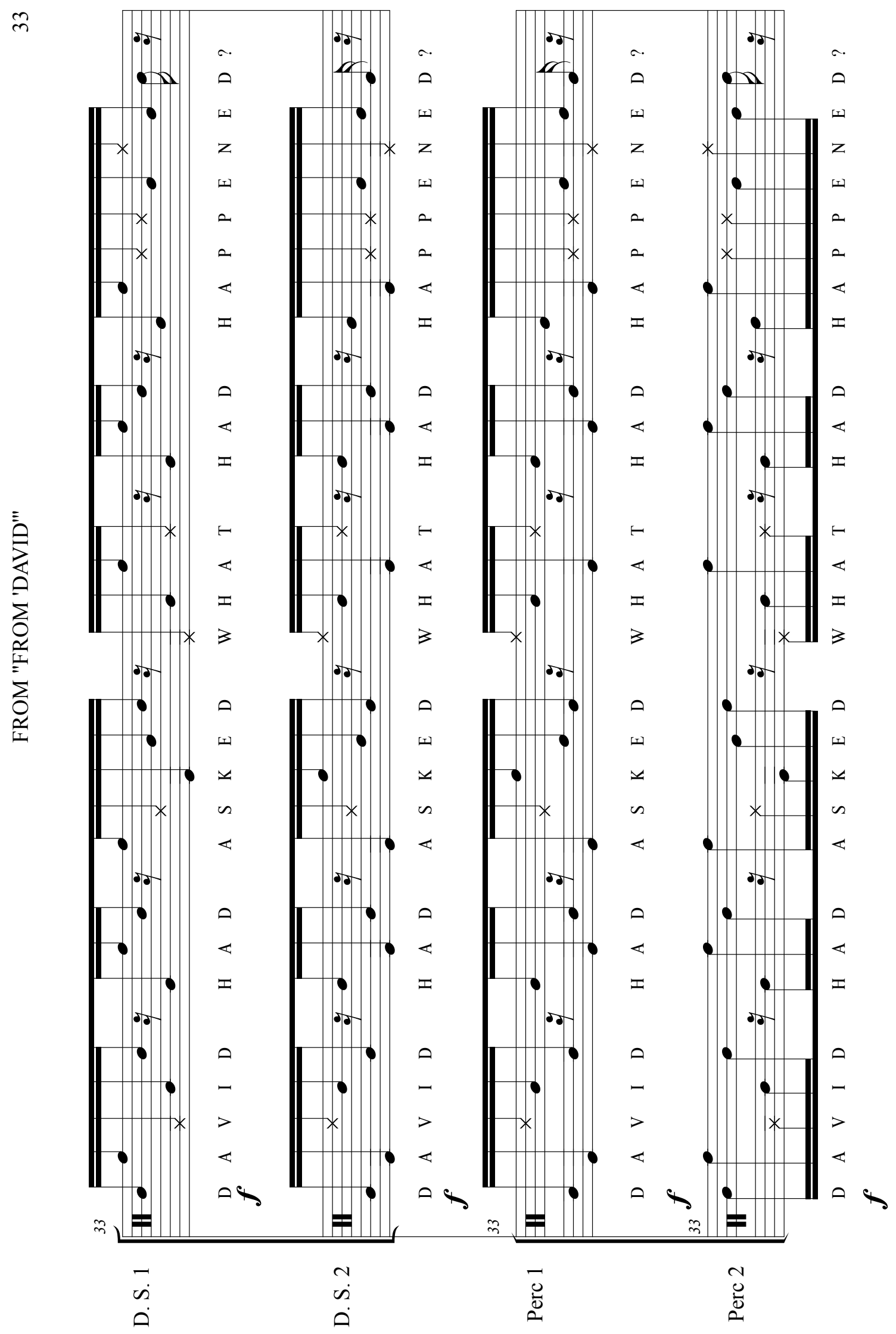




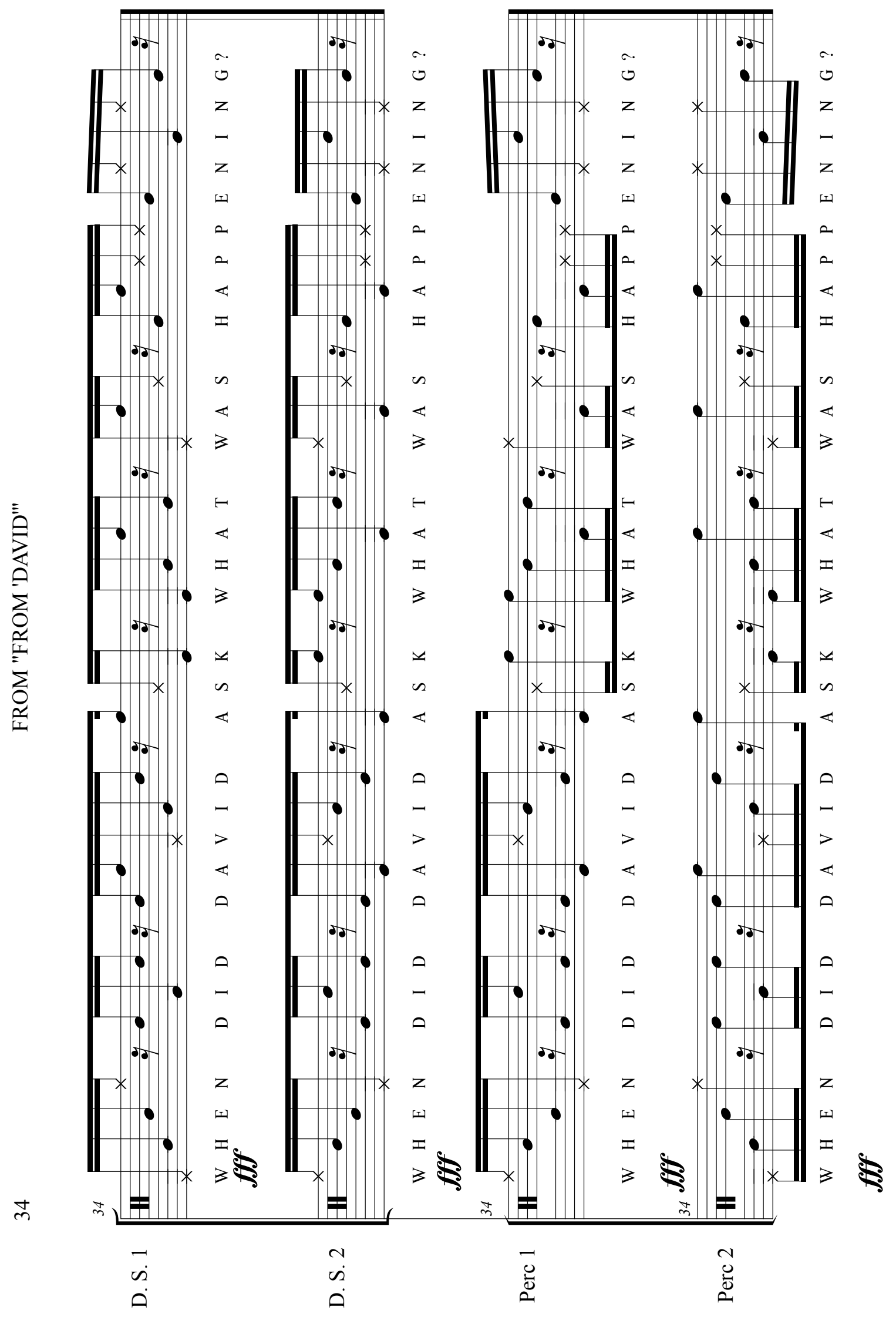




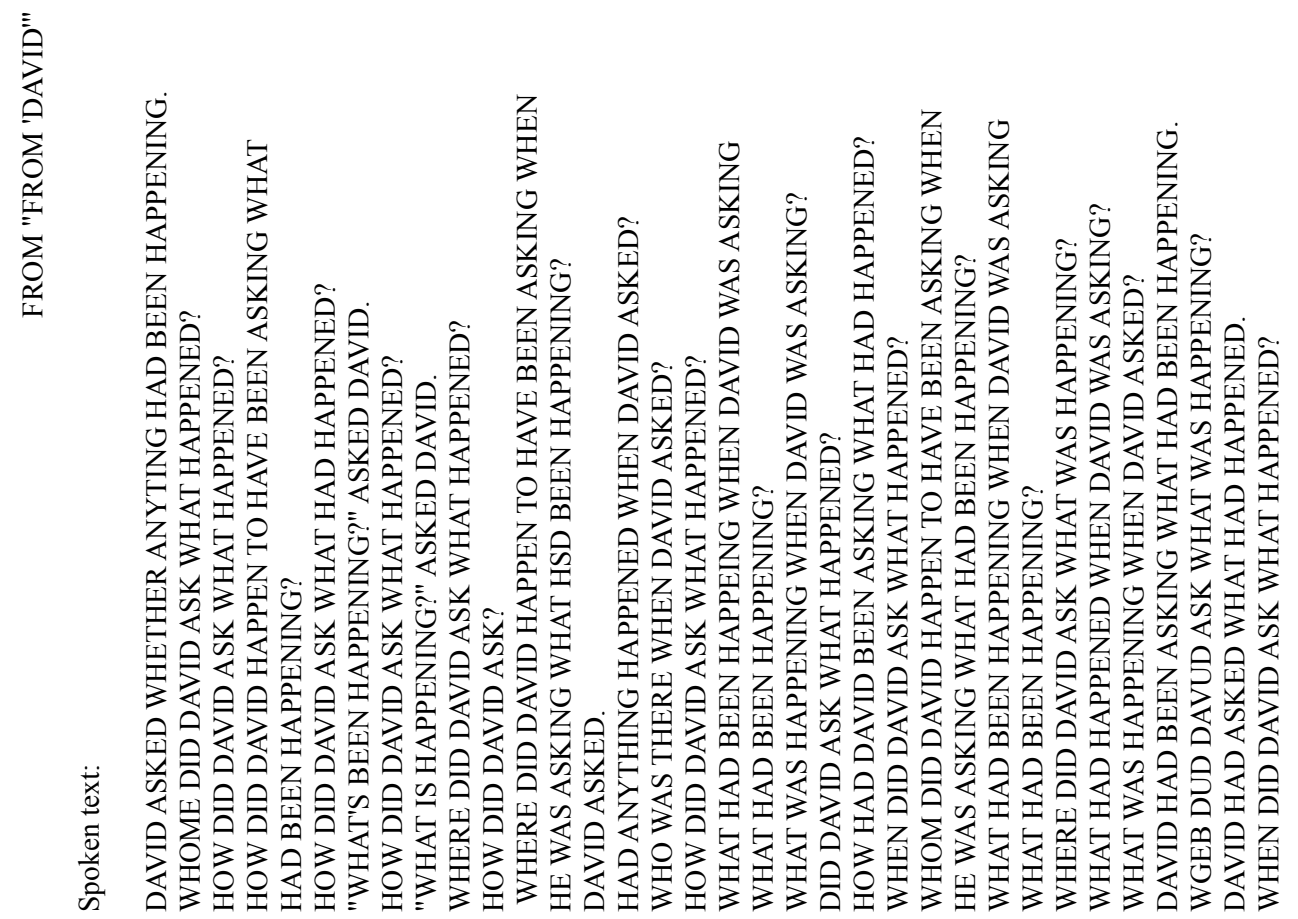


Score

\section{From "From 'South"'}

Kevin William Davis

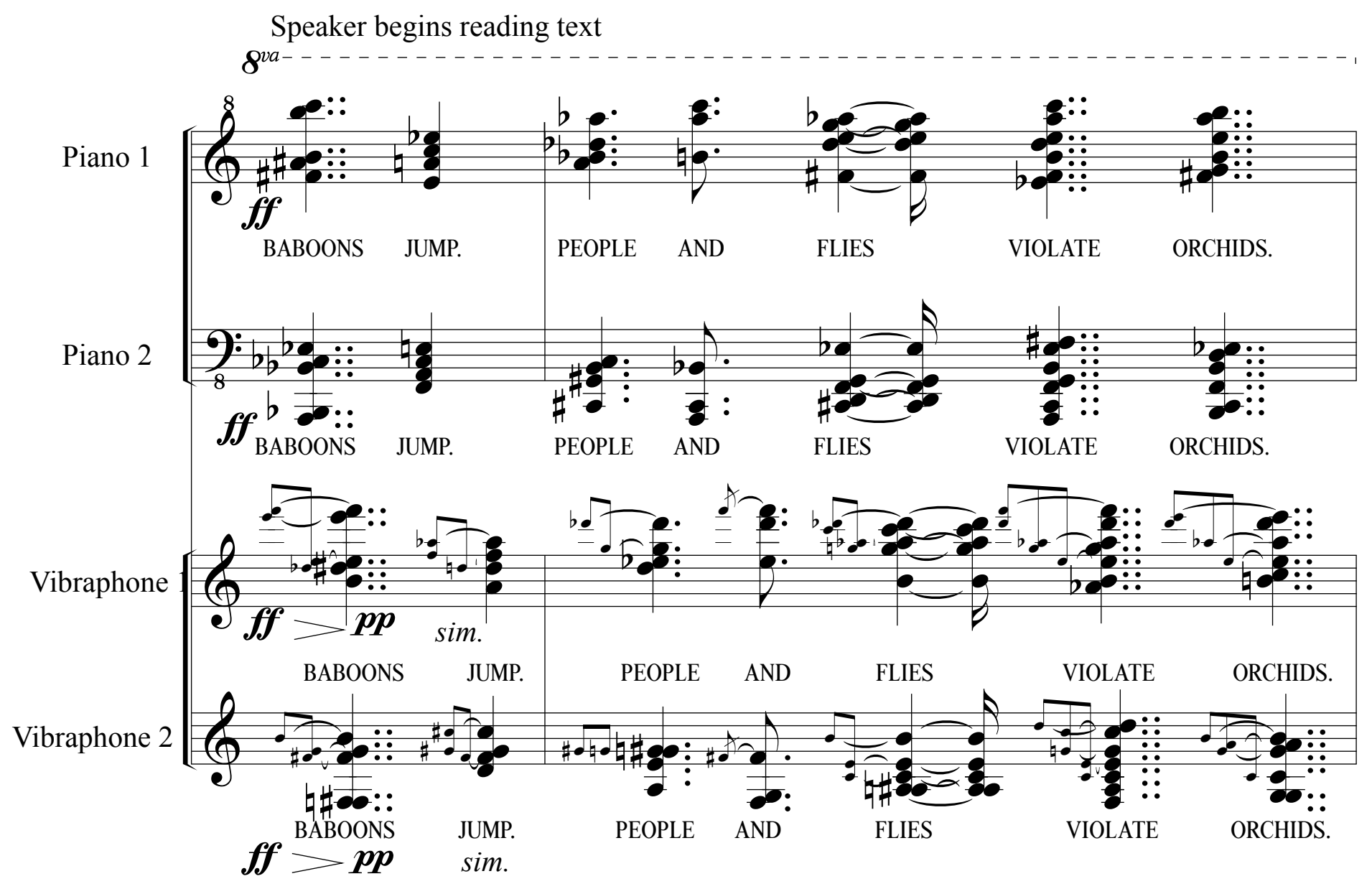

All instruments keep sustain pedals down throughout piece. 
8va sim.

Pf. 1

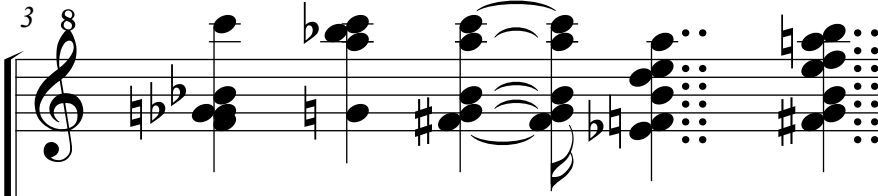

PARROTS RACE ACROSS VIOLET ORCHIDS.

Pf. 2

Vib. 1

Vib. 2

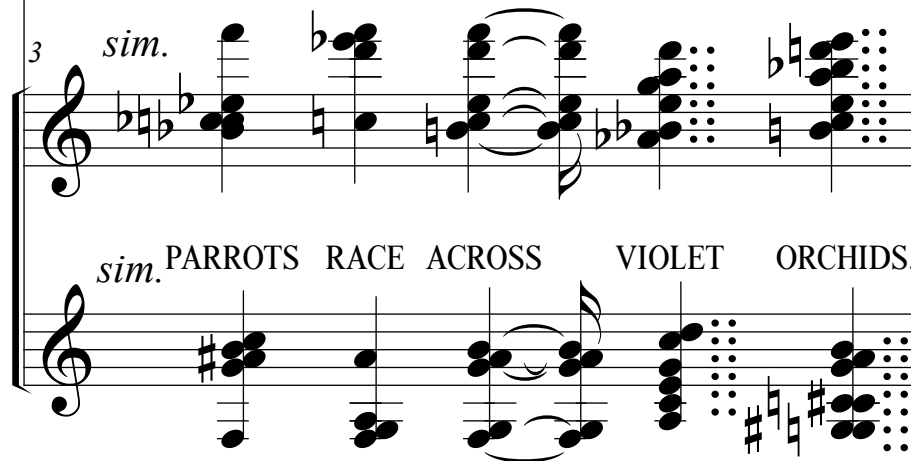

PARROTS RACE ACROSS VIOLET ORCHIDS.

PARROTS RACE ACROSS VIOLET ORCHIDS.

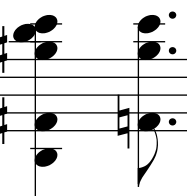

ZEBRAS EAT.

be

$b$ b $\frac{7}{2}$ :

ZEBRAS EAT.
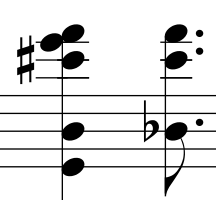

ZEBRAS EAT.

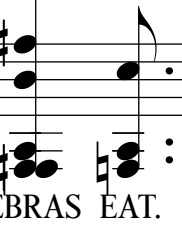

Pf. 1

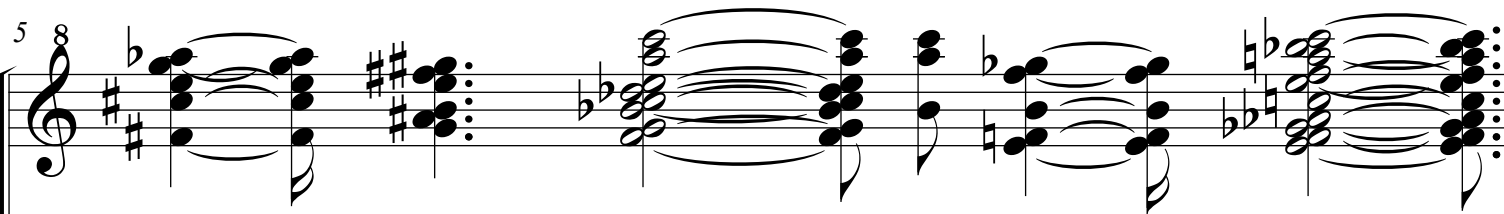

FLIES

IGNORE ARMADILLOS

AND

TOUGH

COATIMUNDIS.

Pf. 2

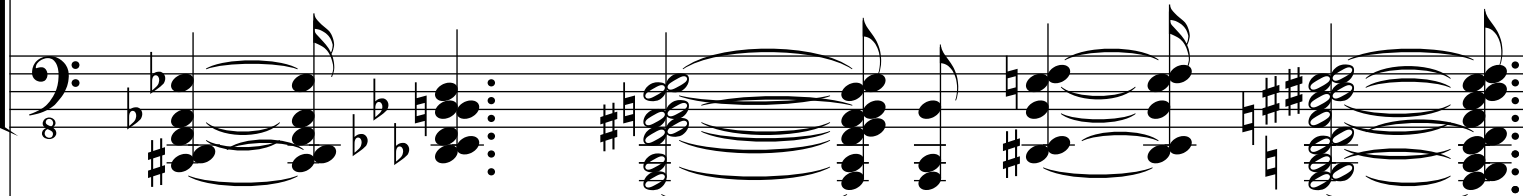

FLIES

IGNORE ARMADILLOS

TOUGH

COATIMUNDIS.

Vib. 1

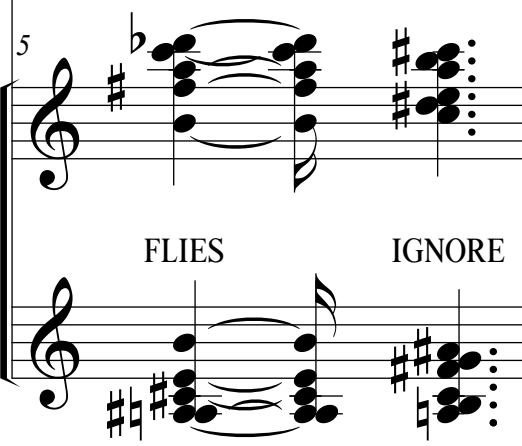

FLIES

IGNORE
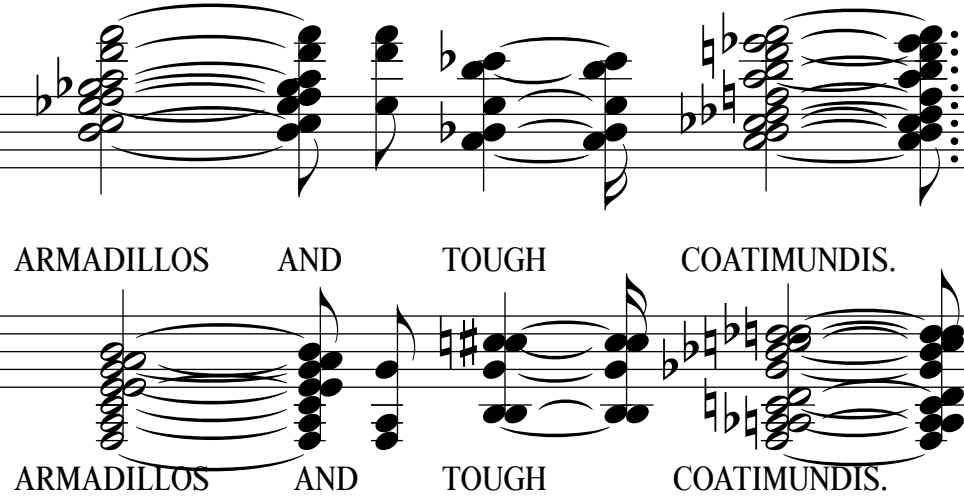

Vib. 2

ARMADILLOS 

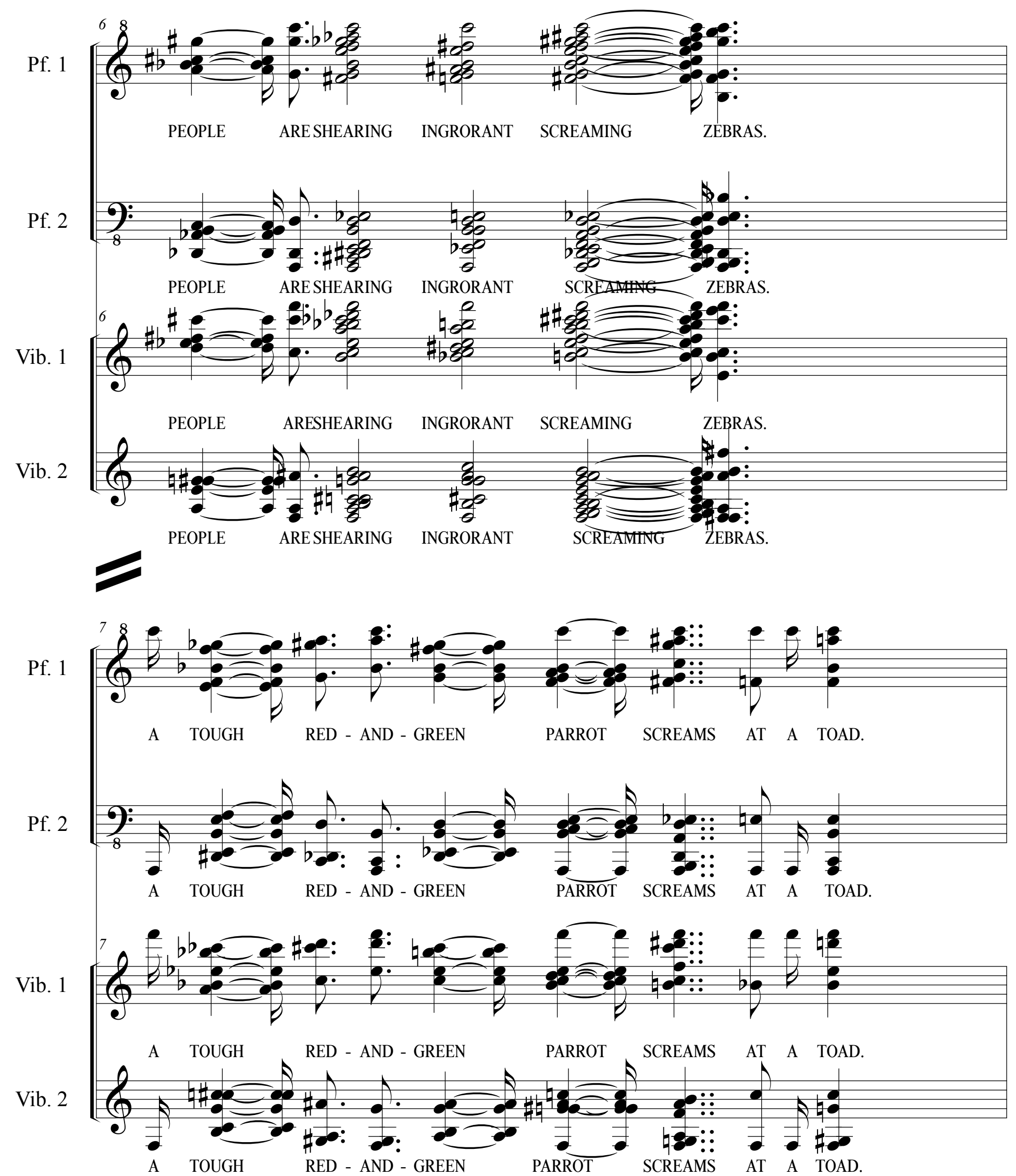


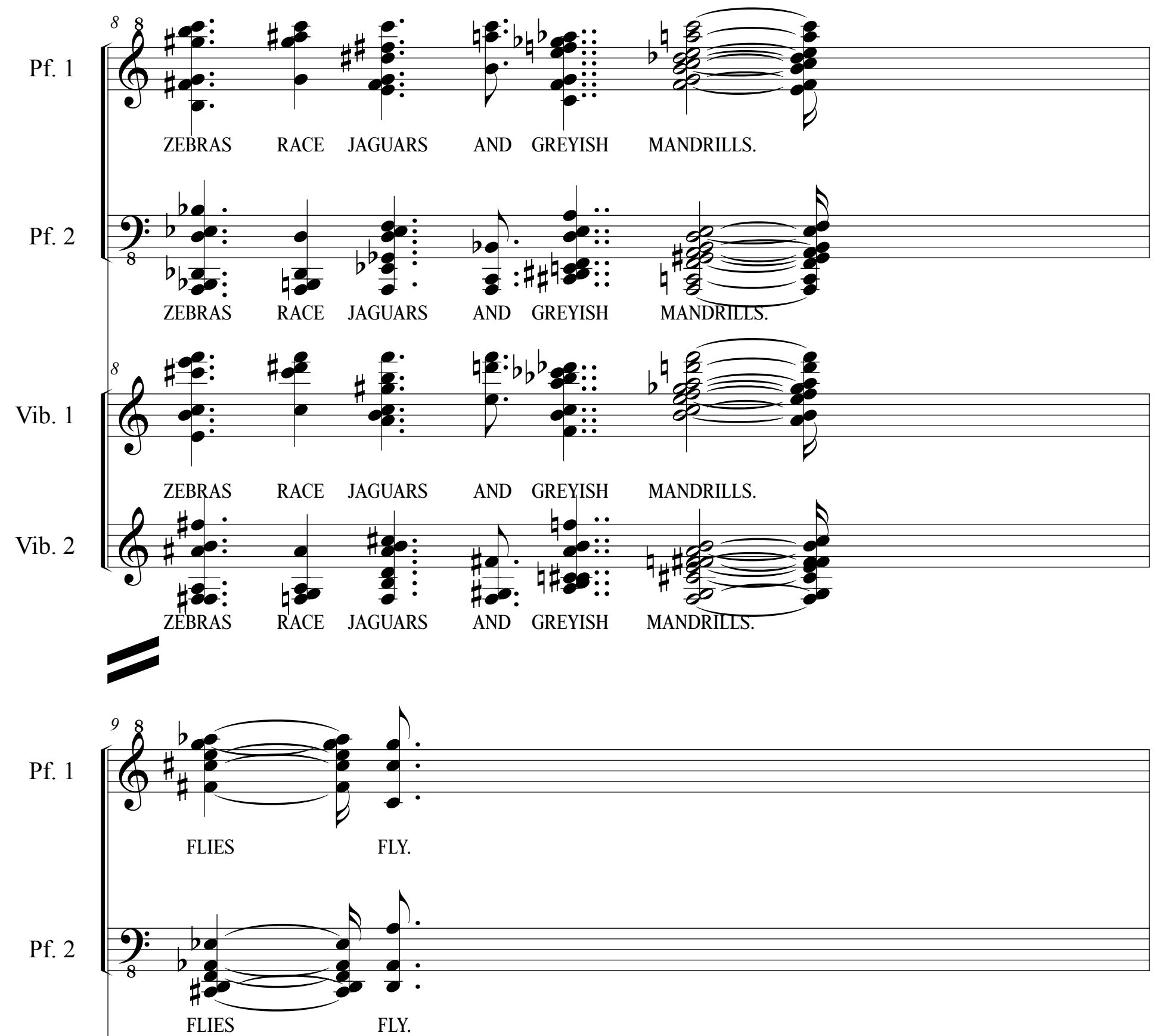

Vib. 1

Vib. 2
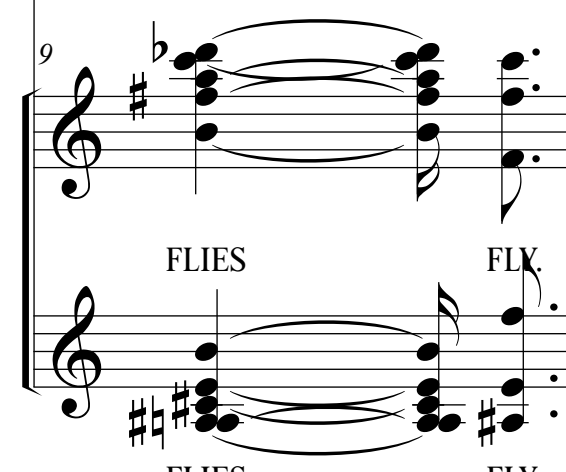

FLIES

FLY. 

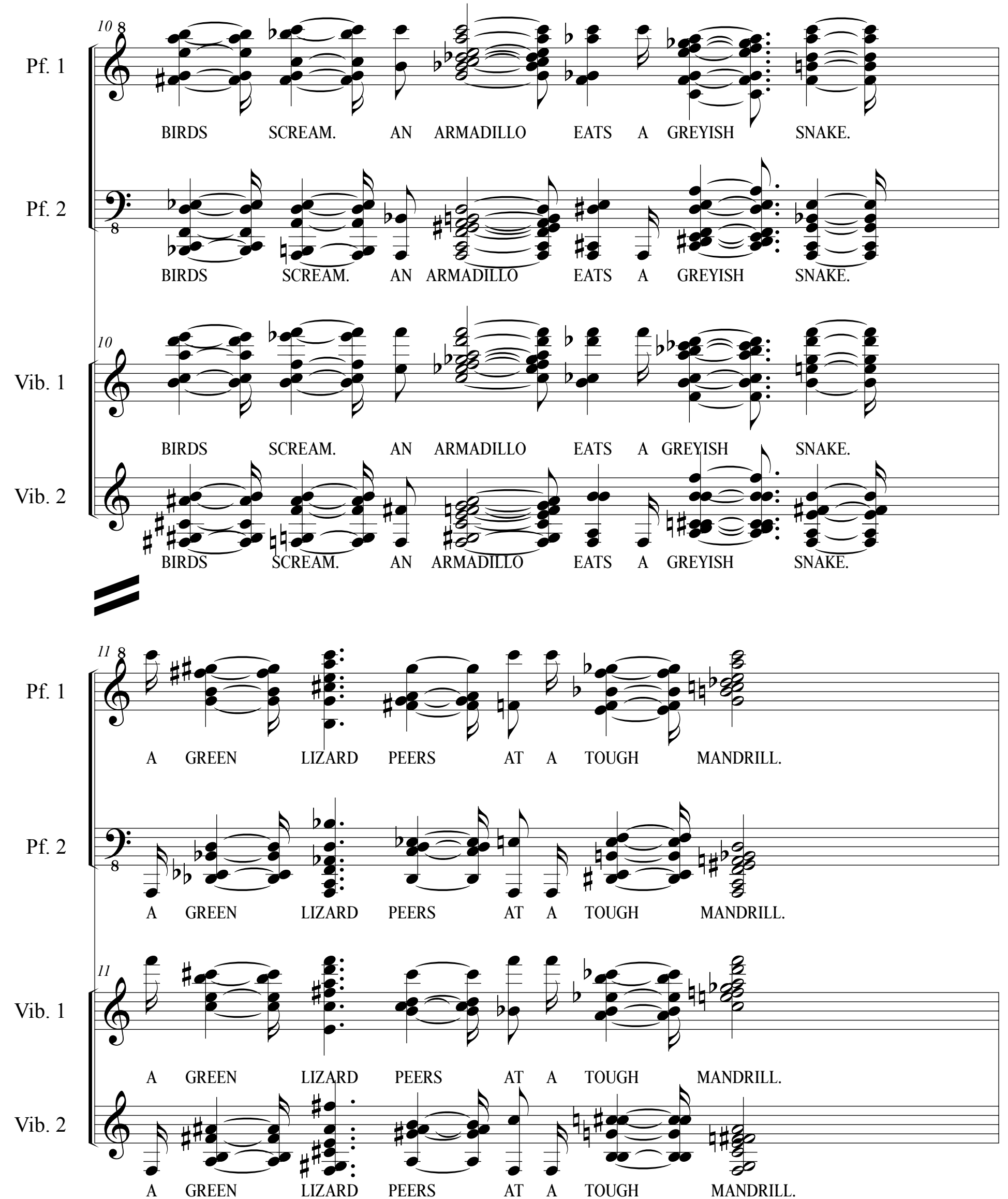

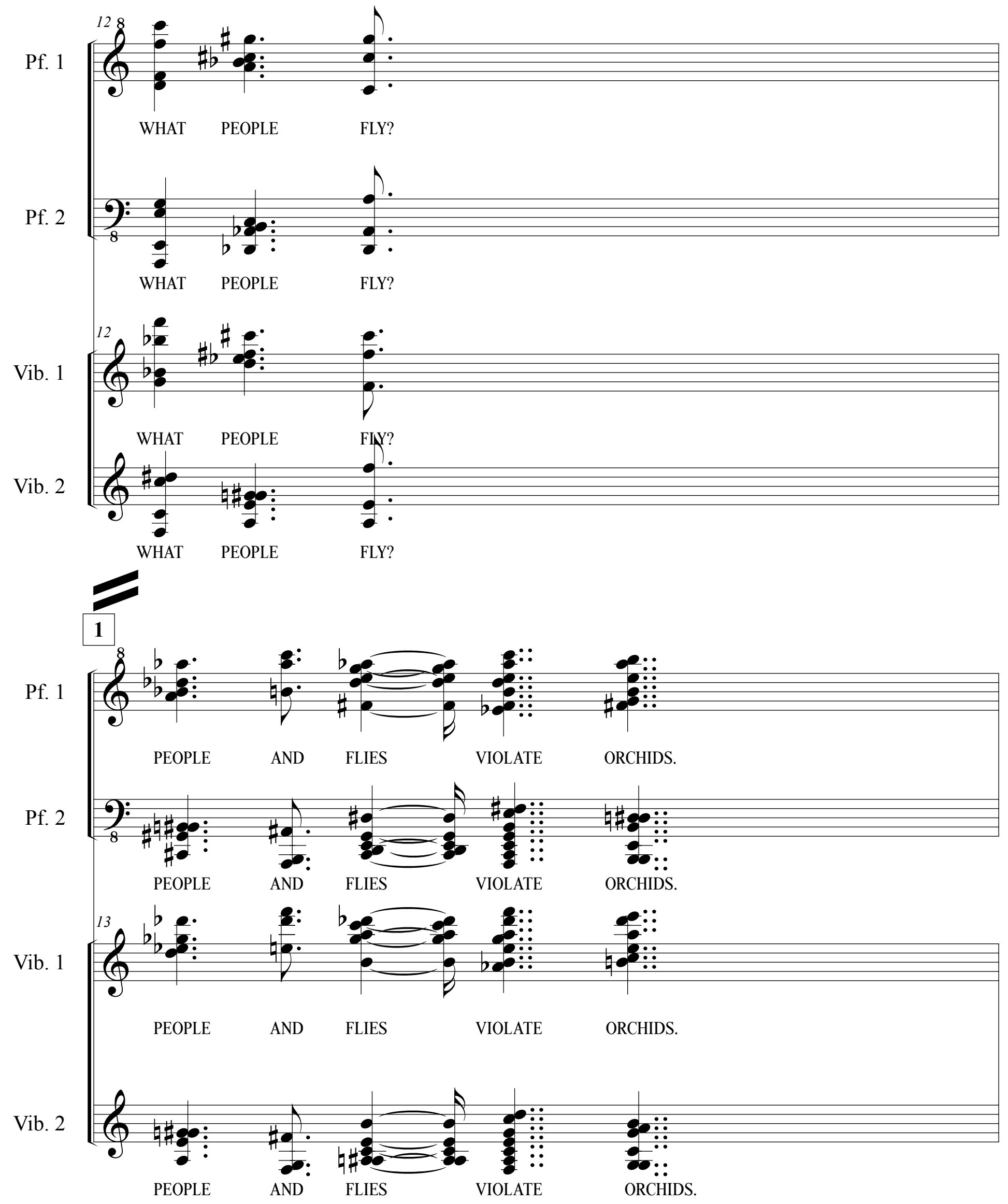

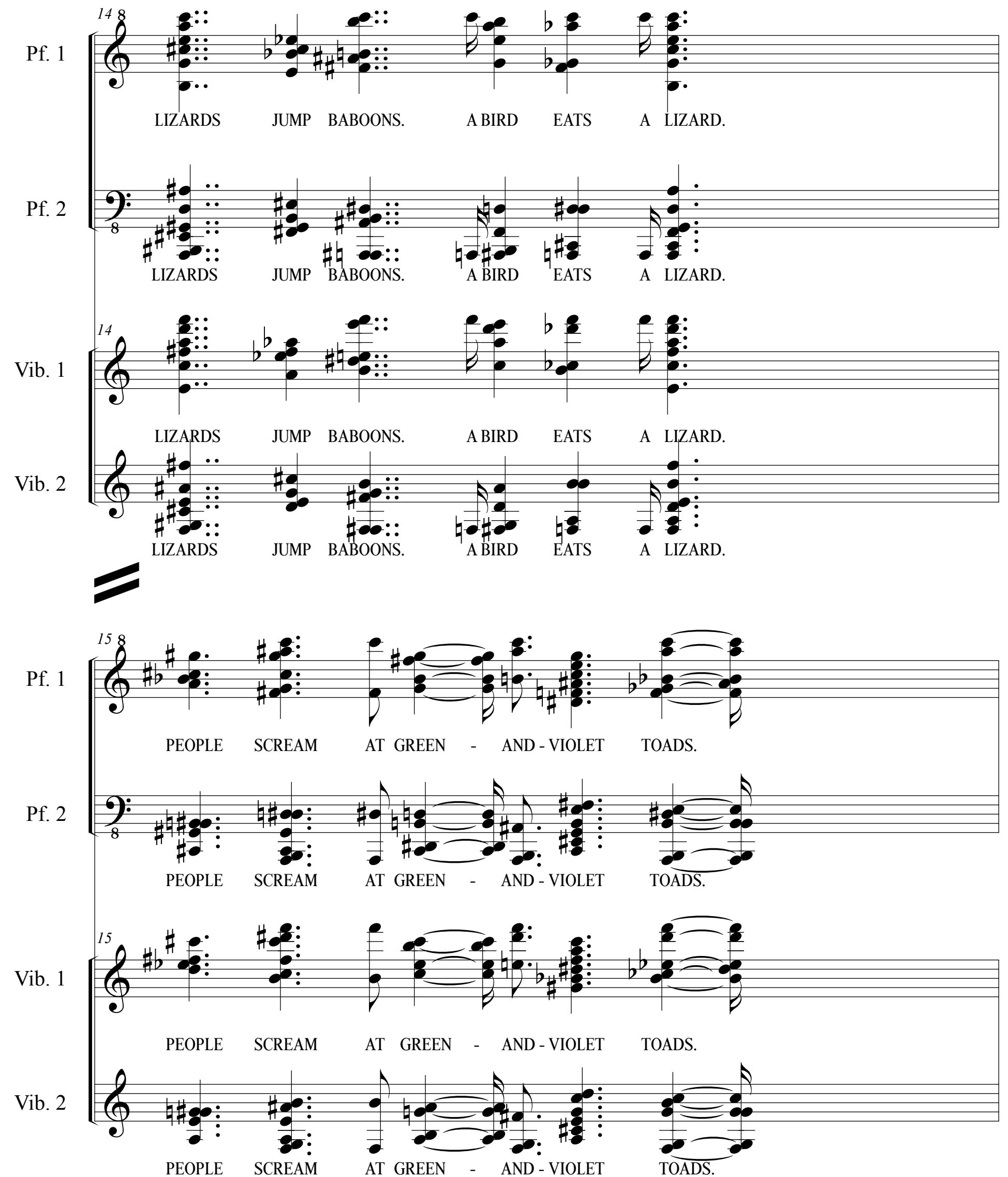

PEOPLE SCREAM

AT GREEN - AND - VIOLET 
Pf. 1

\section{2}

Pf. 2

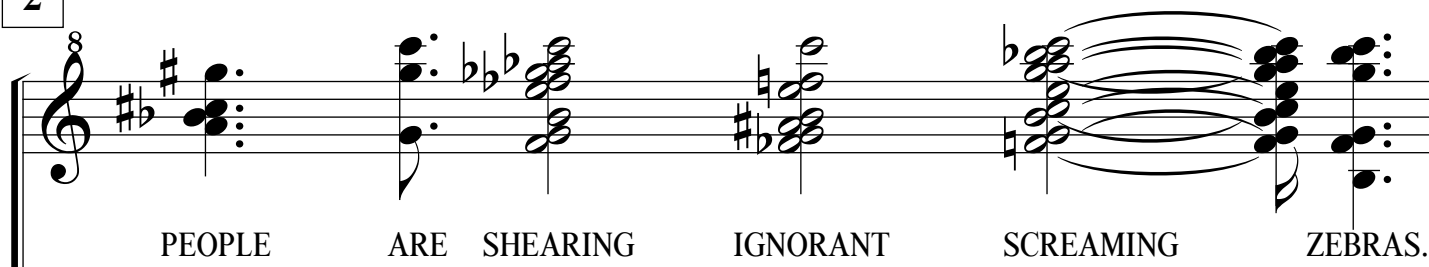

ARE SHEARING

IGNORANT

SCREAMING

ZEBRAS.

Pf. 1

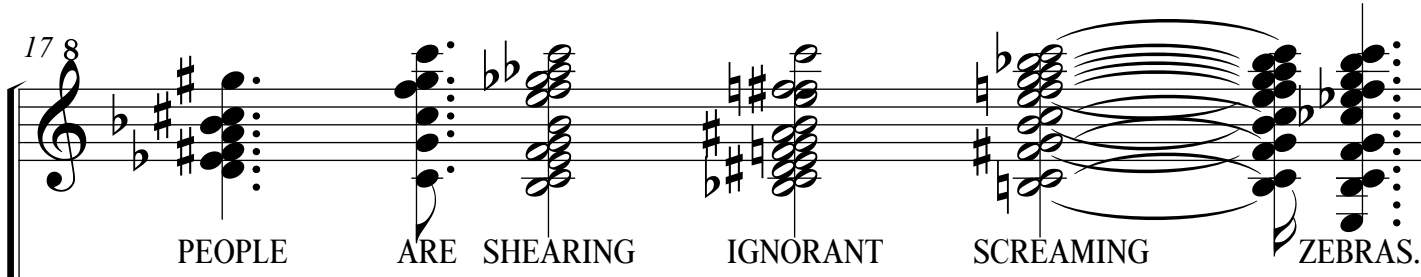

Pf. 2
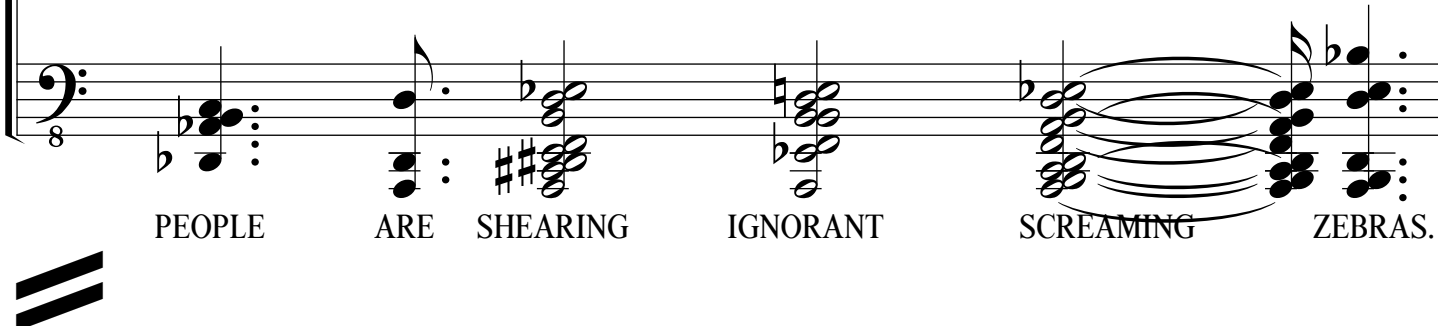

ARE SHEARING

IGNORANT

SCREAMING

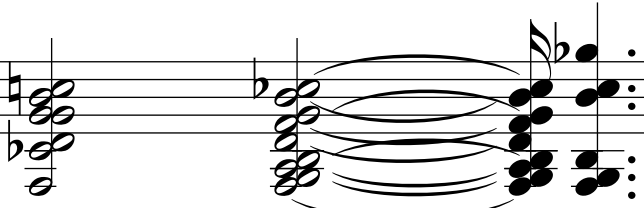

PEOPLE

ARE SHEARING

IGNORANT

SCREAMING

ZEBRAS.

Pf. 2

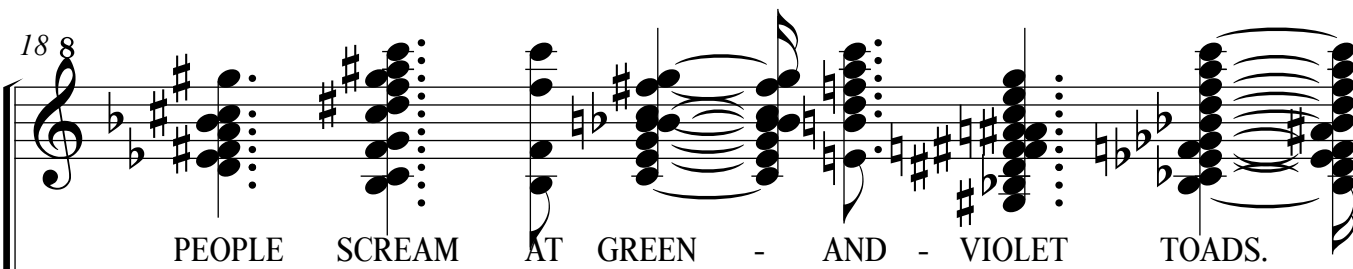

PEOPLE SCREAM AT GREEN - AND - VIOLET TOADS.

\section{Pf. 1}

(9)

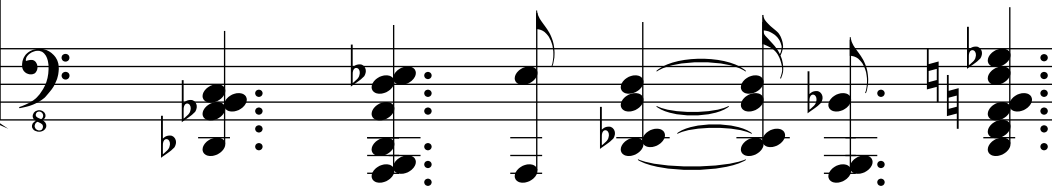

PEOPLE SCREAM

AND - VIOLET

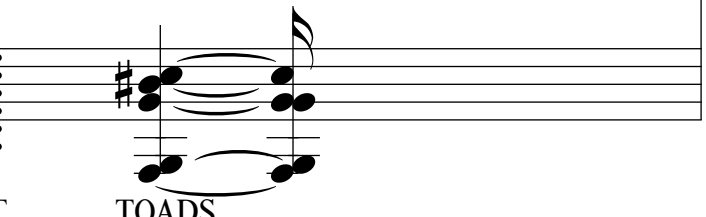


Pf. 1

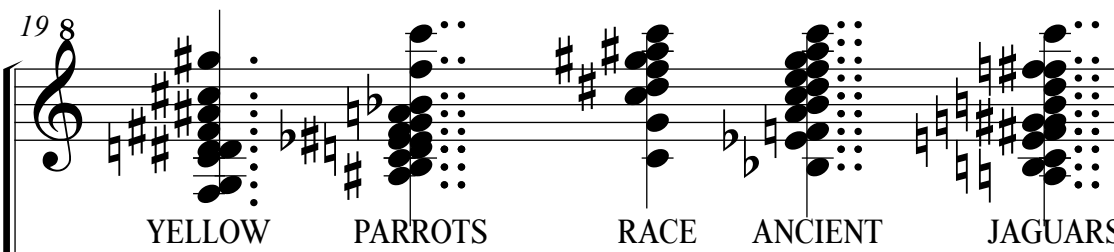

Pf. 2

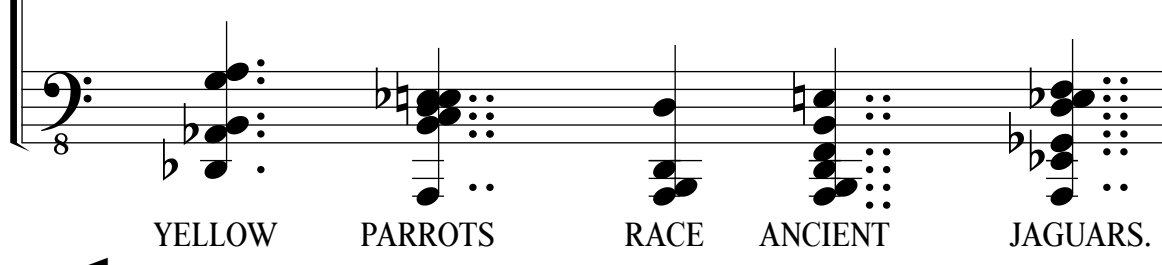

Pf. 1

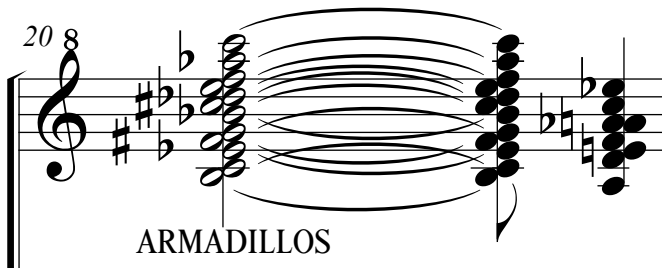

Pf. 2

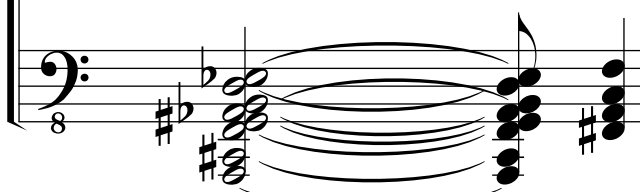

ARMADILLOS

Pf. 1

$$
3
$$

Pf. 2
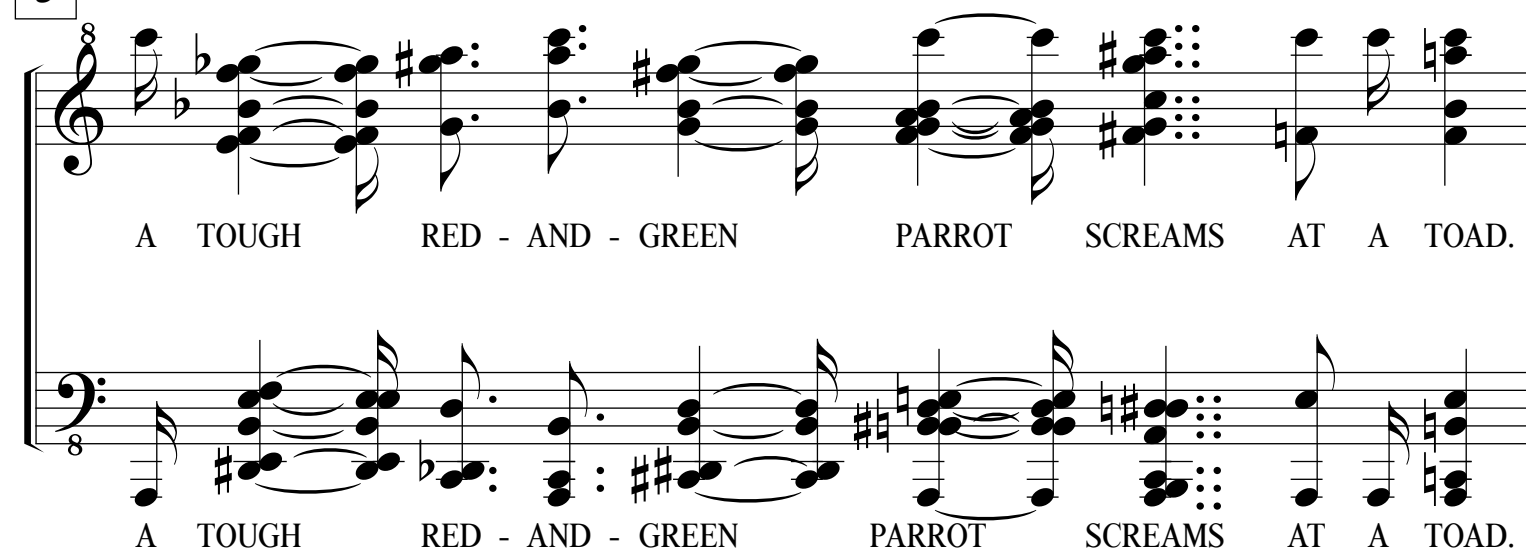

A TOUGH RED - AND - GREEN

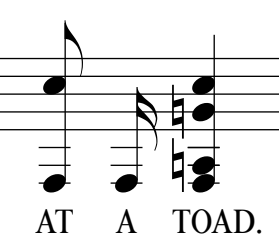


Pf. 1

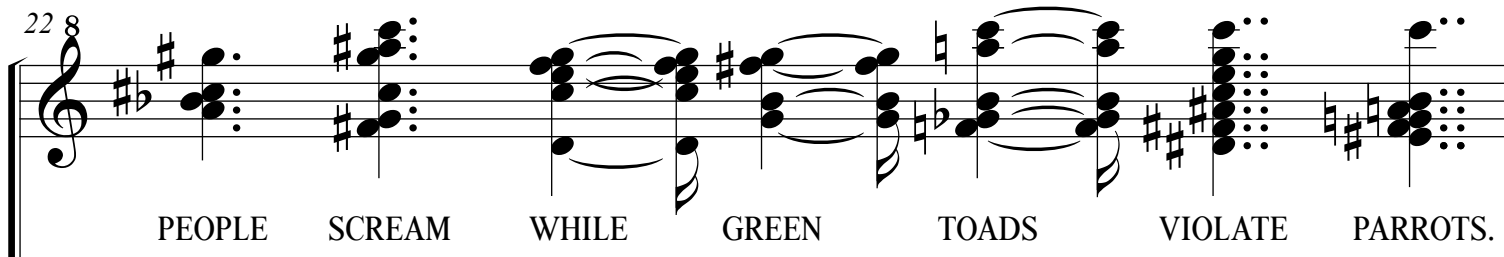

Pf. 2

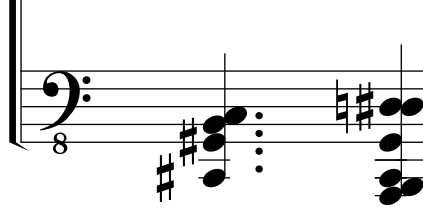

PEOPLE SCREAM WHILE

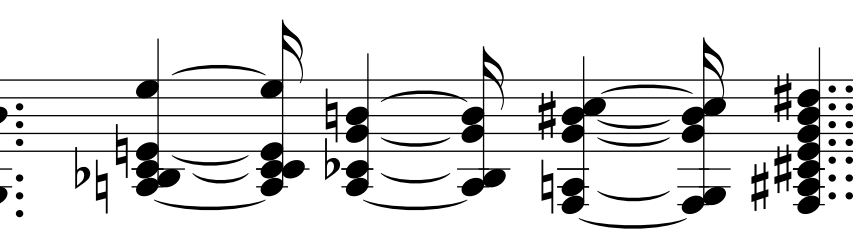

GREEN
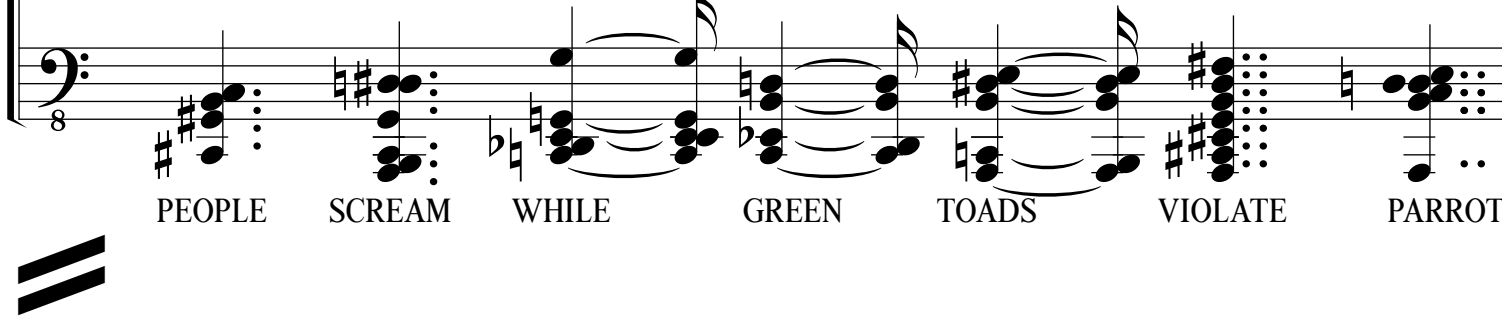

Pf. 1

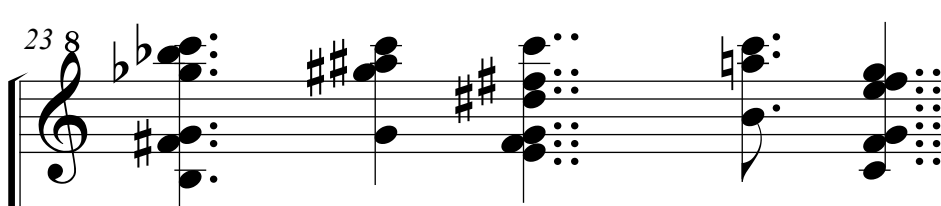

ZEBRAS

RACE JAGUARS

AND GREYISH

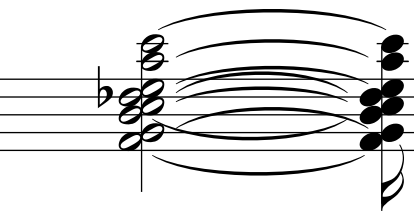

MANDRILLS.

Pf. 2
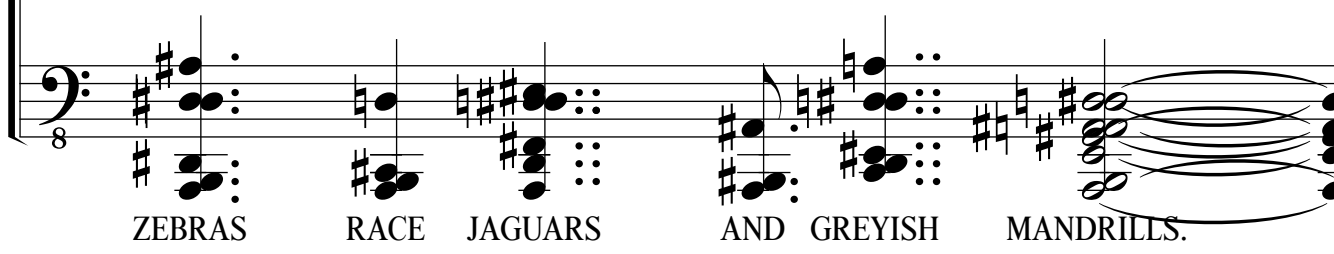

MANDRILLS.

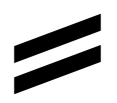

4

Pf. 1

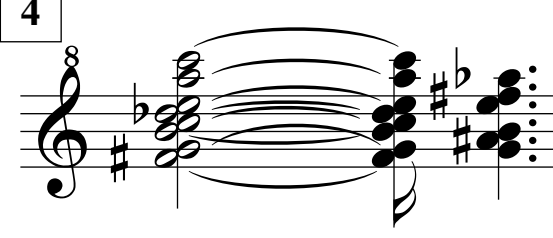

MANDRILLS

IGNORE

ARMADILLOS

COATIMUNDIS 
Pf. 1

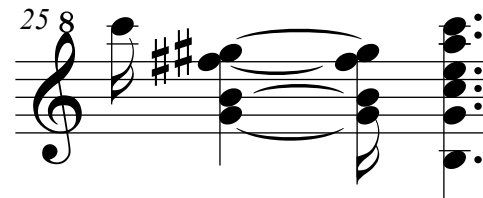

A GREEN

LIZARD

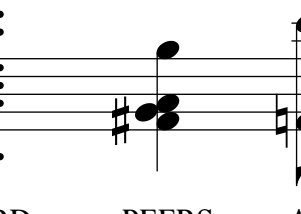

PEERS

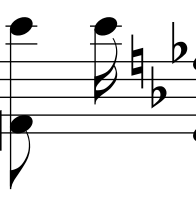

AT A TOUGH

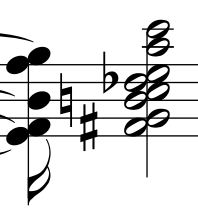

MANDRILL.

Pf. 1
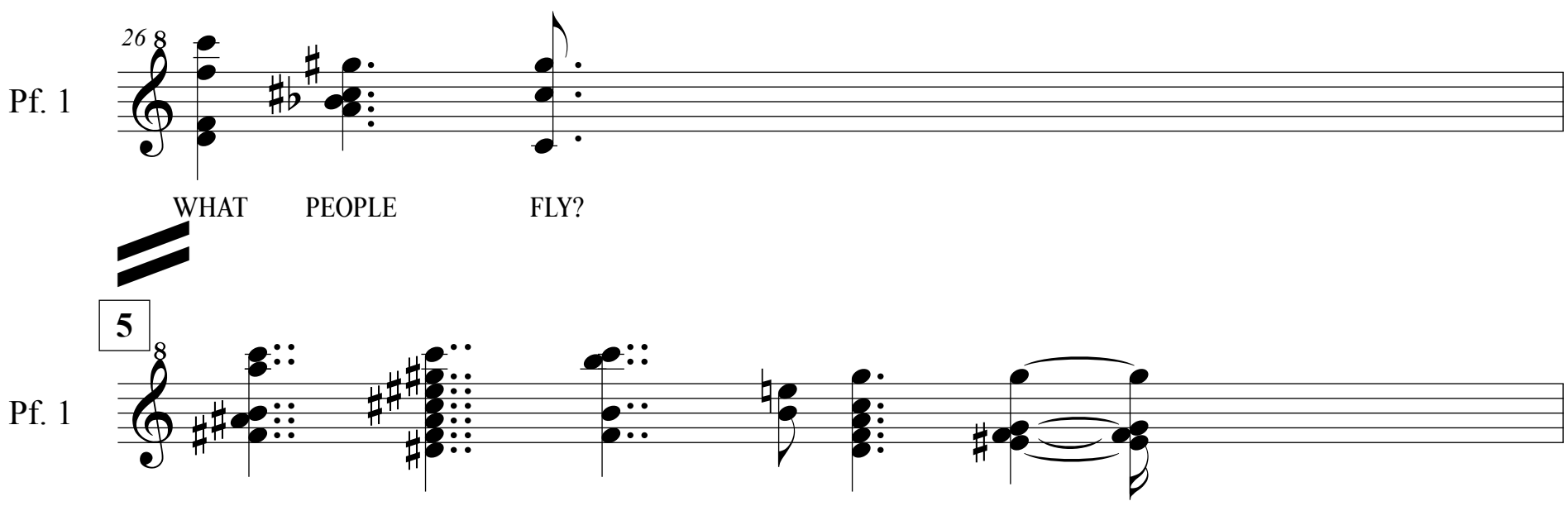
BABOONS VIOLATE BANANAS IN VIOLET TREES.

Pf. 1

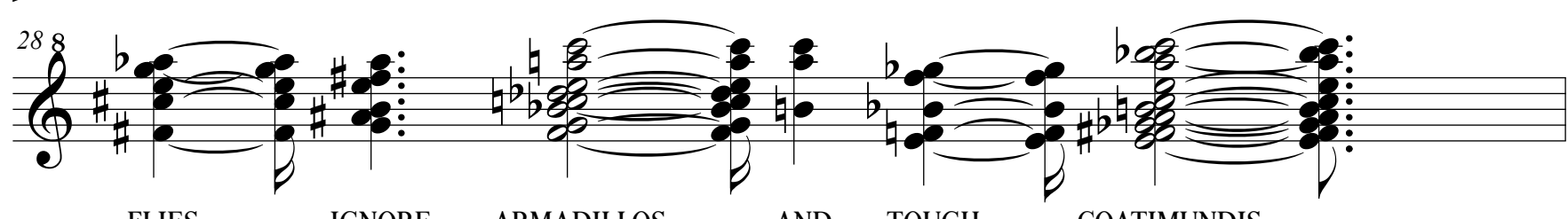

FLIES

IGNORE ARMADILLOS

AND TOUGH

COATIMUNDIS.

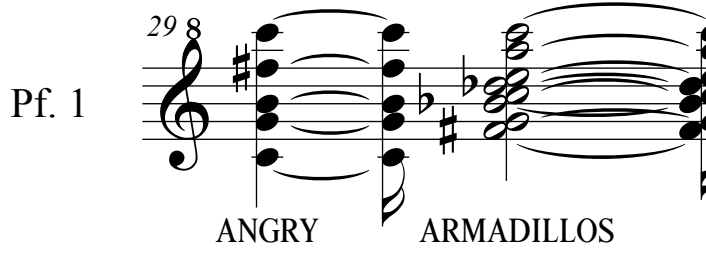
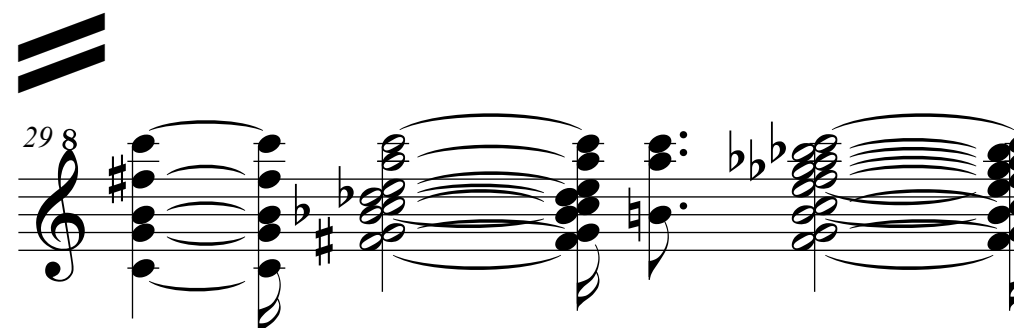

ARE SCREAMING

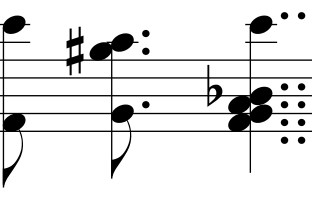

AT RED PARROTS. 
Pf. 1
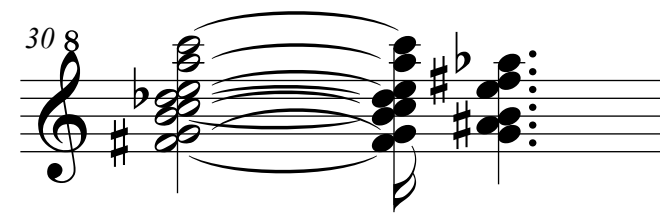

MANDRILLS

IGNORE

ARMADILLOS

AND COATIMUNDIS.

Pf. 1

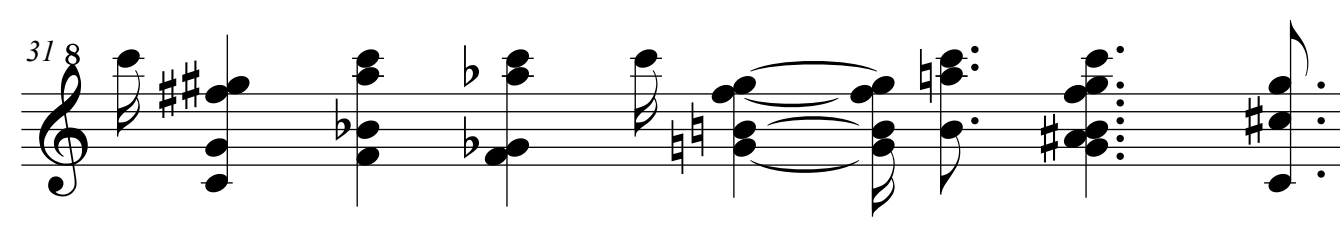

Pf. 1
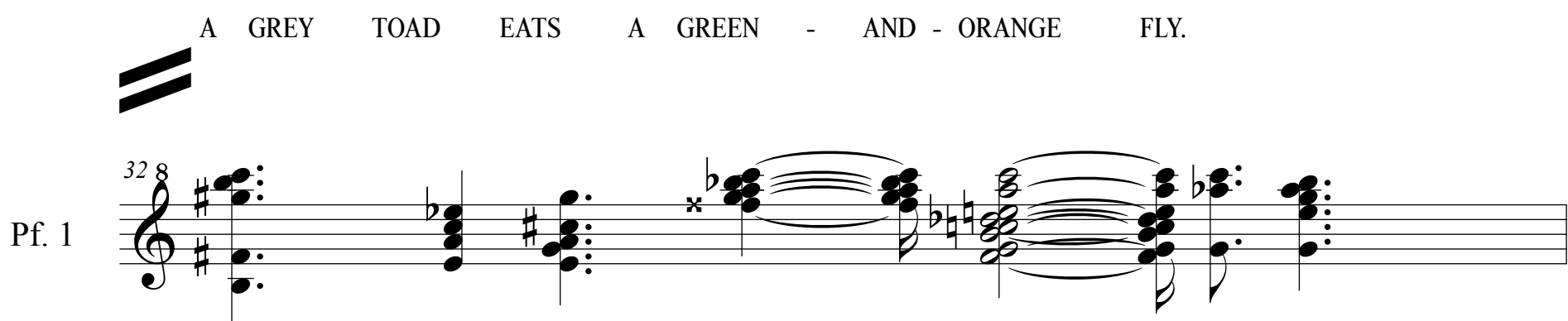
ZEBRAS
JUMP. PURPLE
FACED
MANDRILLS
ARE FIERCE.

Pf. 1

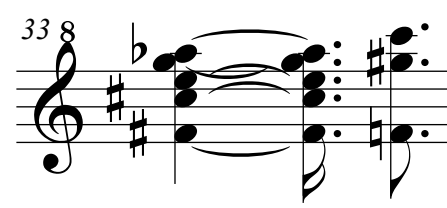

FLIES

EAT.

Pf. 1

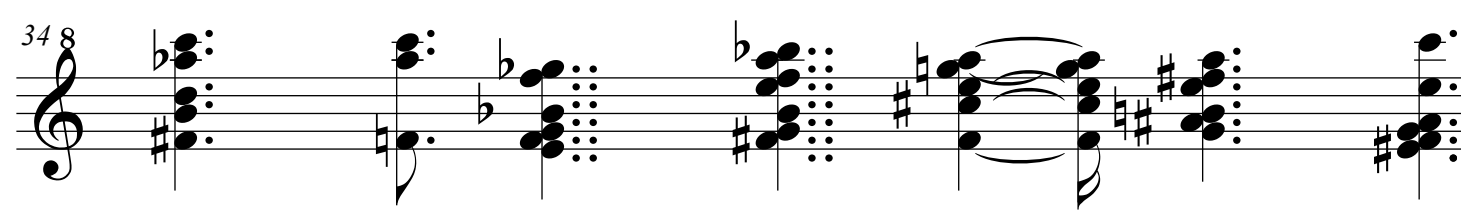

SNAKES EAT THROUGH ORCHIDS. FLIES IGNORE TAPIRS.

Pf. 1

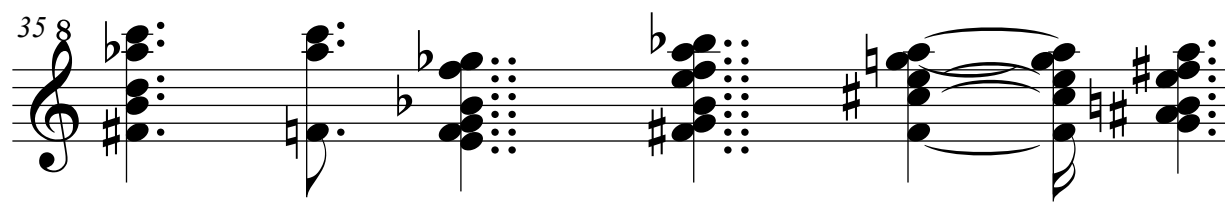

SNAKES EAT THROUGH ORCHIDS.

FLIES

IGNORE TAPIRS. 
Score

\title{
From "From 'The""
}

\author{
Kevin William Davis
}

In this piece, the indicated text is spoken and the given shapes are to be traced out in the manner indiacted. All gestures/rhytms should be at a relaxed, speech-like pace. Parts should only stay loosly aligned. Each bracket indicates a new section. In the first section speaker and players start evey line together with pauses in between. In all subsequent sections, the speaker and percussionist begin the section together but need not stay together. The speaker cues each entrance.

The overall dynamic is quiet. This piece can be patient with substantial pauses between sections. Percussion IV/keyboard should hold down the sustain pedal for the entire piece while playing snare drum until section 11.

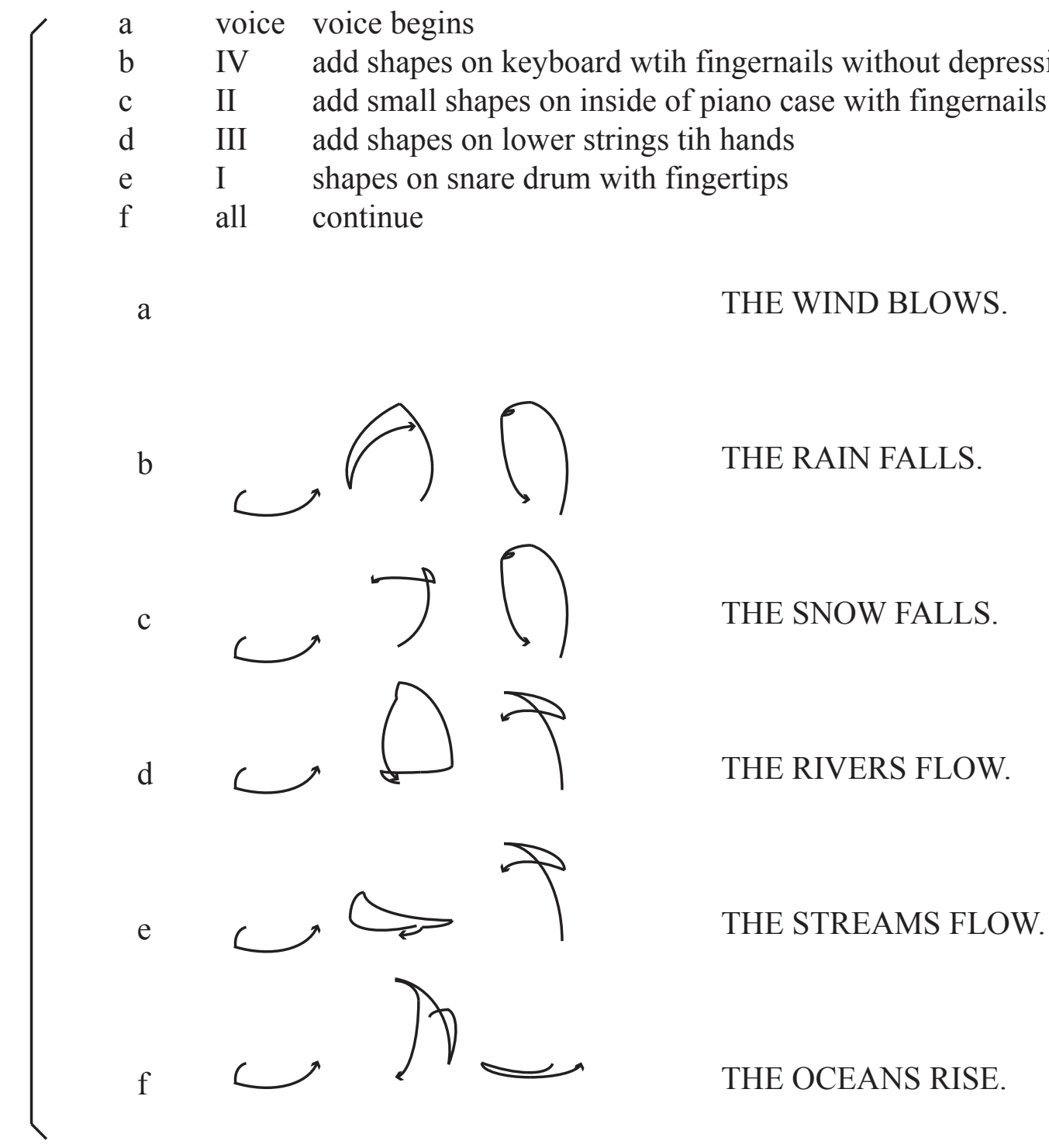




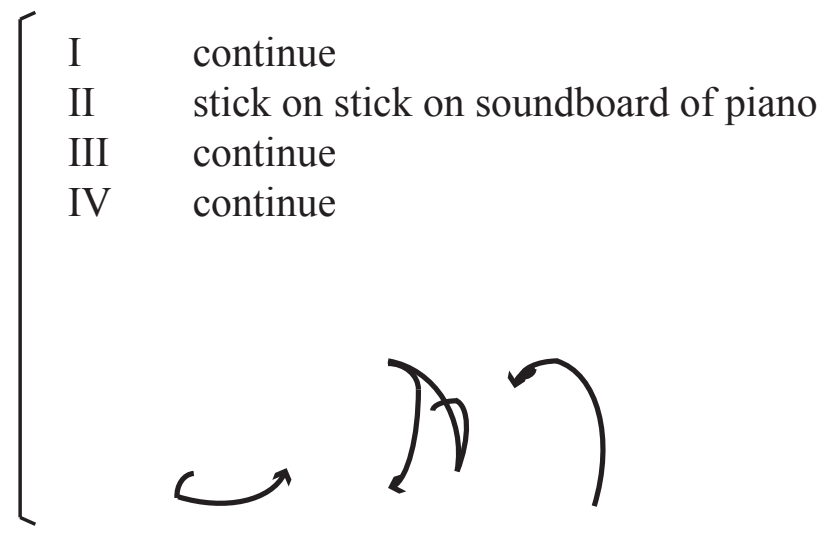

THE OCEANS FALL.

I continue

II continue

III wire brush in middle section

IV continue

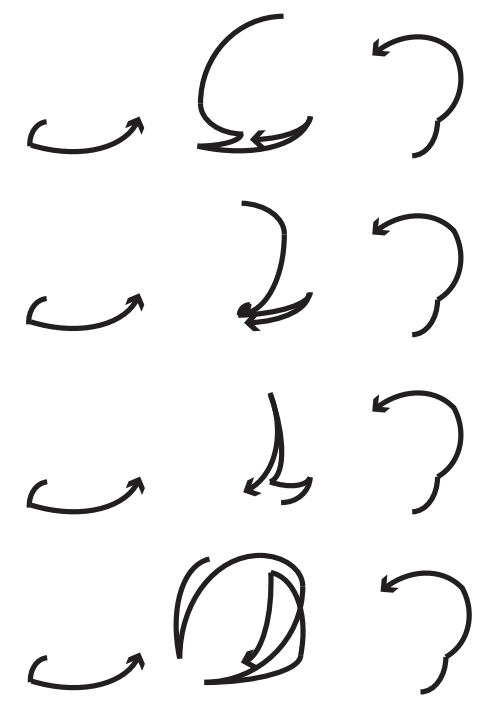

THE BUSHES GROW.

THE MOSSES GROW.

THE FERNS GROW.

THE LICHENS GROW. 

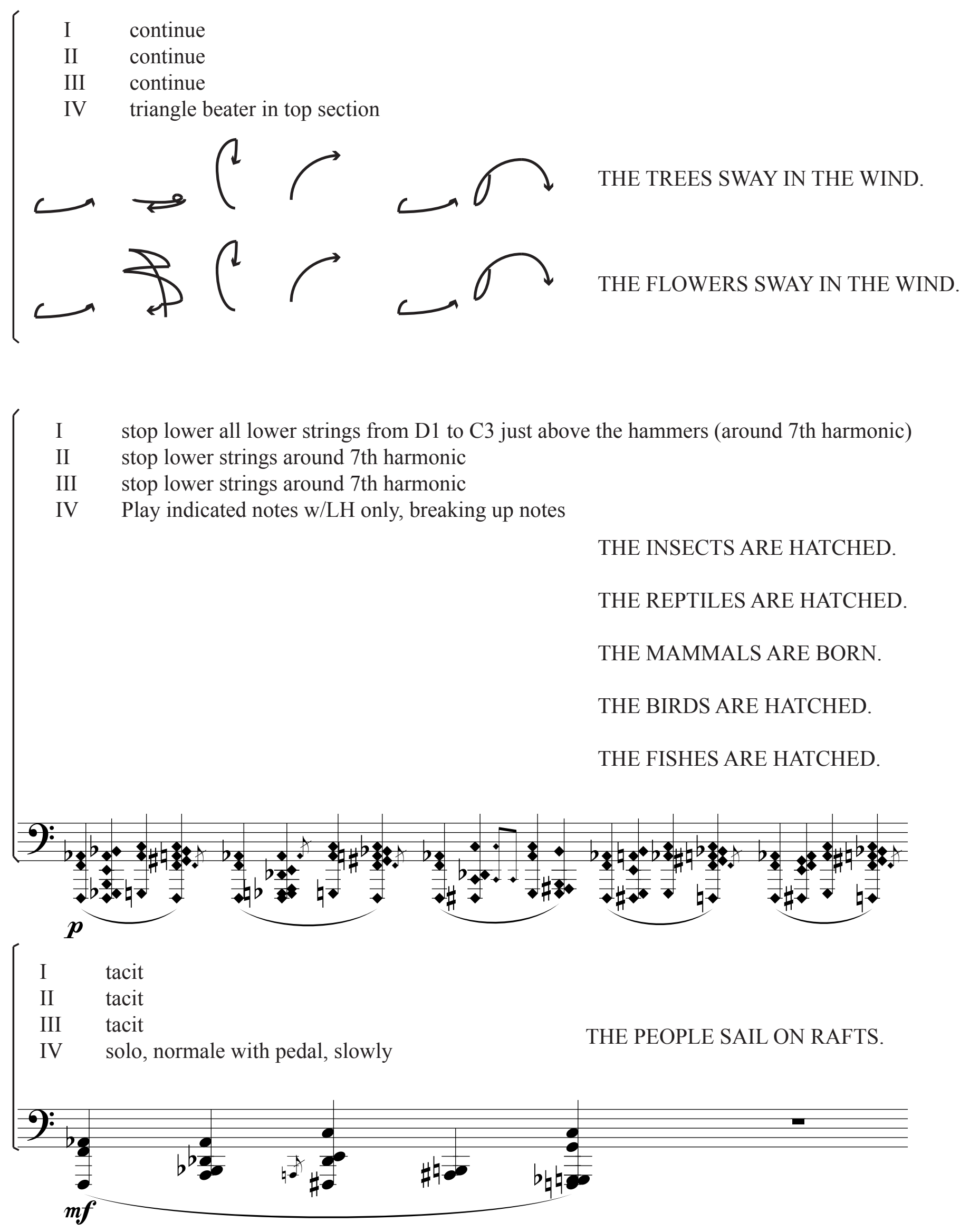

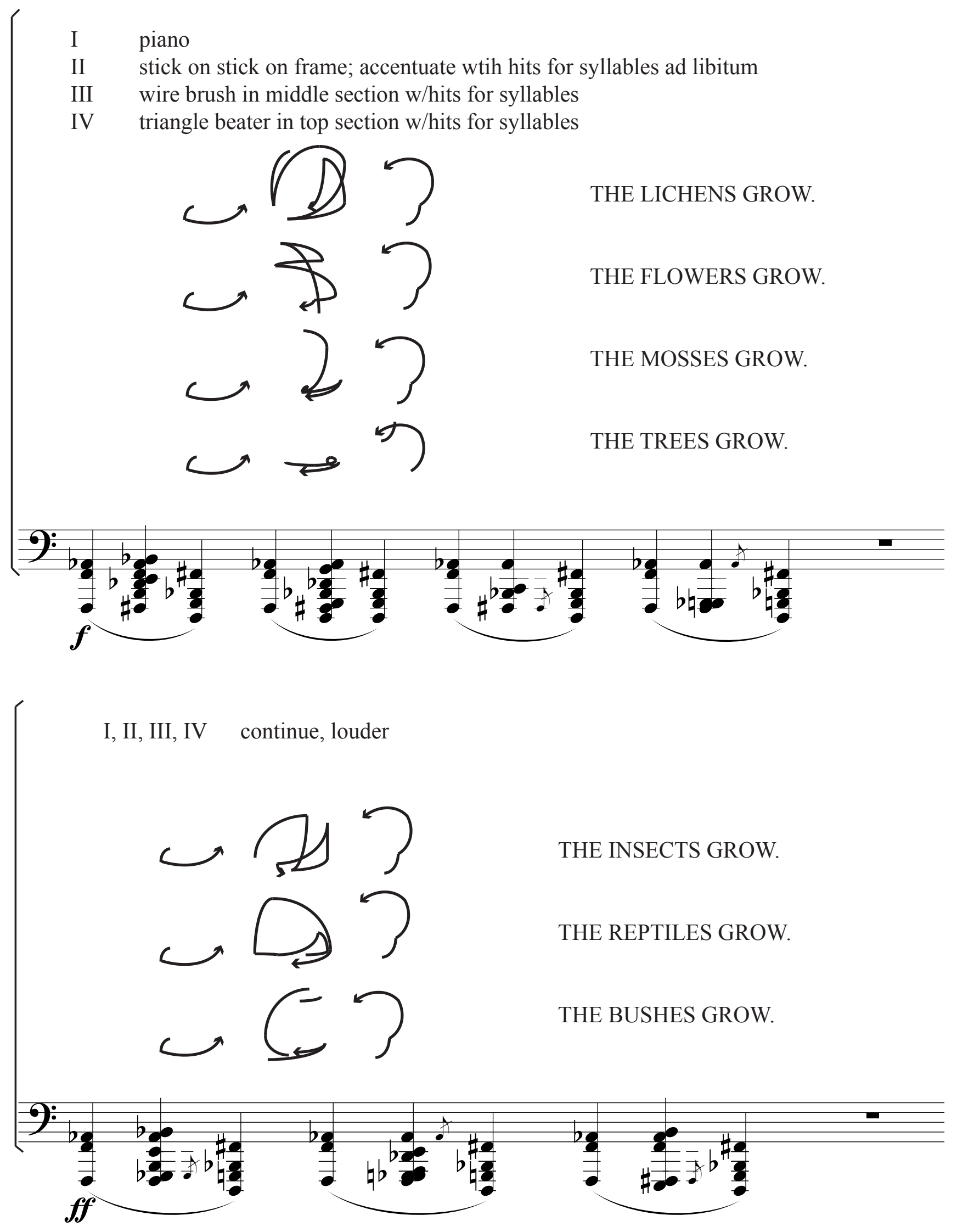

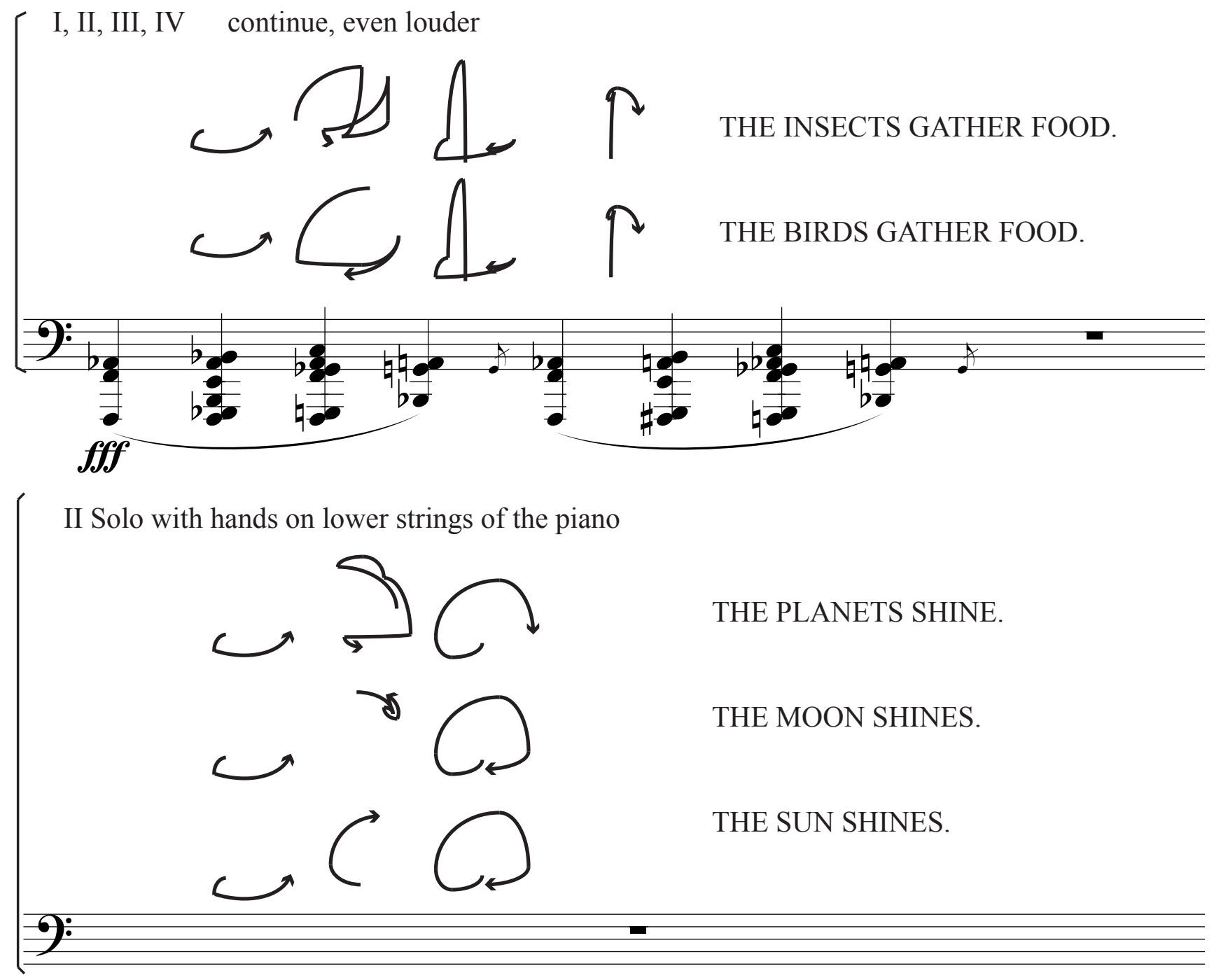

Slowly release pedal after percussion II solo

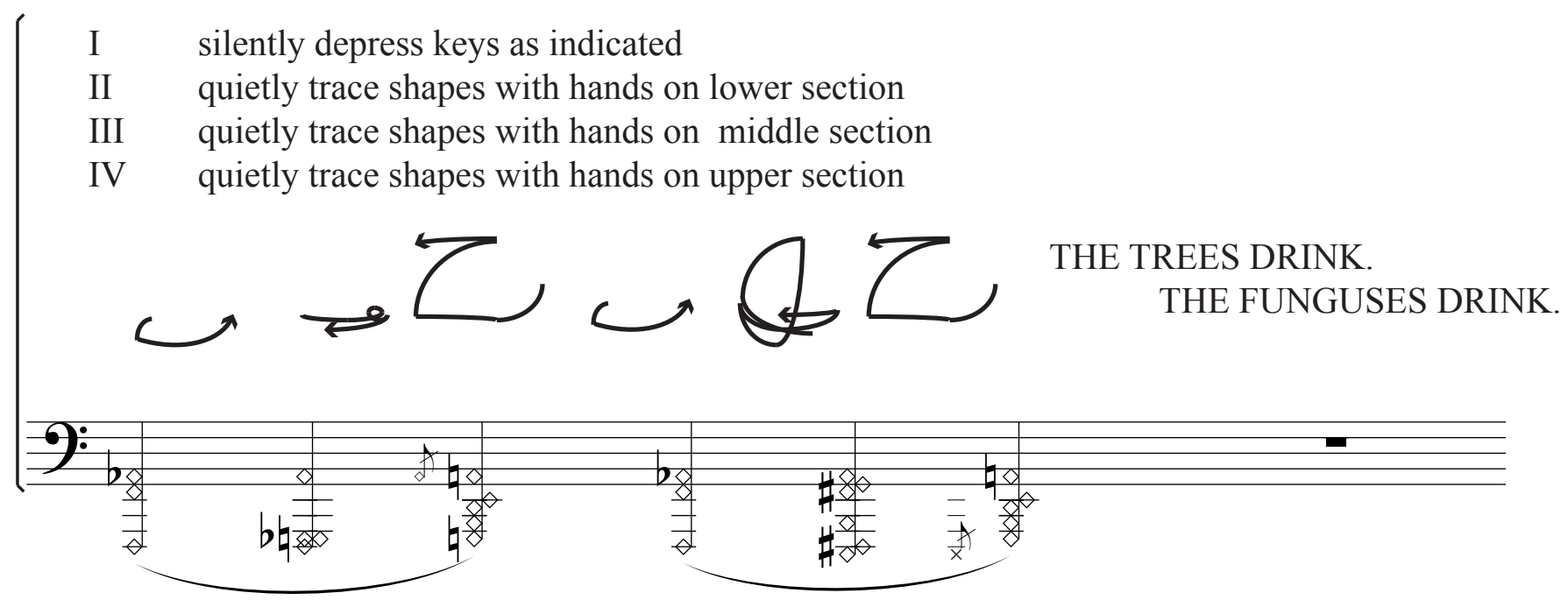

Silently depress keys while other percussionists play inside piano 


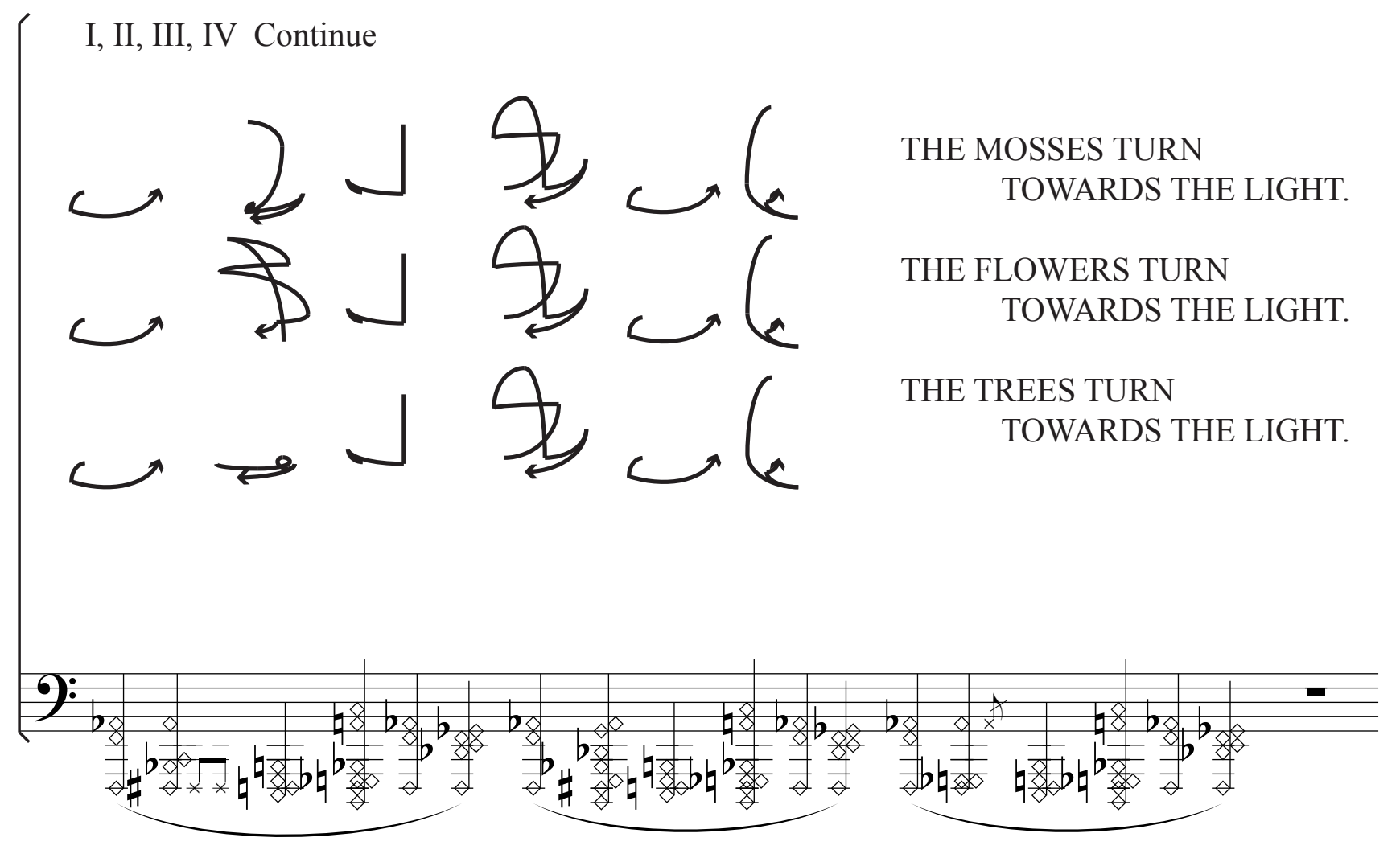




\section{Bibliography}

Ablinger, Peter. "Black Square and Bottle Rack: Noise and Noises." In Noise in and as Music, edited by Aaron Cassidy and Aaron Einbond, 5-8. Huddersfield: University of Huddersfield Press, 2013.

Adorno, Theodor W. Philosophy of New Music. Translated by Robert Hullot-Kentor. Minneapolis: University of Minnesota Press, 2006.

- - . "Vers Une Musique Informelle." In Quasi Una Fantasia: Essays on Modern Music, 269-322. London, New York: Verso, 1992.

Adorno, Theodor W, Gretel Adorno, and Rolf Tiedeman. Aesthetic Theory. Theory and History of Literature. Minneapolis: University of Minnesota Press, 1997.

Akrich, Madeleine, and Bruno Latour. "A Summary of a Convenient Vocabulary for the Semiotics of Human and Nonhuman Assemblies." In Shaping Technology, Building Society: Studies in Sociotechnical Change, edited by Wiebe E. Bijker and John Law, 259-64. Cambridge, MA and London: The MIT Press, 1992.

Alburger, Mark. "Lemonte Young to 1960." 21st Century Music 10, no. 3 (n.d.): 3-9.

Anthony DiFlorio. Jimmy Durante A Piano Is a Delicate Thing 44 70. Accessed April 22, 2017. https://www.youtube.com/watch?v=sGiT-uJR4ZY.

Armstrong, Newton. "An Enactive Approach to Digital Musical Instrument Design.” PhD dissertation, Princeton University, 2006.

Attali, Jacques. Noise: The Political Economy of Music. Translated by Brian Massumi. Theory and History of Literature. Minneapolis: University of Minnesota Press, 1985.

Bailey, Derek. Improvisation: Its Nature and Practice in Music. Ashborune, England: Da Capo Press, 1992.

Bartee, Jordan. "Ontological Toys." PhD dissertation, Brown University, 2014.

Bathes, Roland. "The Grain of the Voice." In Image Music Text, translated by Stephen Heath, 179-89. London: Fontana Press, 1977.

Bayar, Tildy. "Music Inside Out: Spectral Music's Chords of 'Nature.” Spectral World Musics, October 2008, $107-18$.

Belting, Hans. The Invisible Masterpiece. London: Reaktion, 2001.

Bennett, Jane. Vibrant Matter: A Political Ecology of Things. Durham: Duke University Press, 2010.

Benson, Bruce Ellis. The Improvisation of Musical Dialogue: A Phenomenology of Music. Cambridge, New York: Cambridge University Press, 2003.

Berliner, Paul. The Soul of Mbira: Music and Traditions of the Shona People of Zimbabwe. Perspectives on Southern Africa ; 26. Berkeley: University of California Press, 1978.

- - . Thinking in Jazz: The Infinite Art of Improvisation. Chicago Studies in Ethnomusicology. Chicago: University of Chicago Press, 1994.

Bernstein, J. M. Against Voluptuous Bodies: Late Modernism and the Meaning of Painting. Stanford, CA: Stanford University Press, 2006.

Bogue, Ronald. “Scoring the Rhizome: Bussotti’s Musical Diagram.” Deleuze Studies 8, no. 4 (2014): 470-90. doi:10.3366/dls.2014.0166.

Britt, Neil Cameron. “Actuated Acoustic Instruments: Relationships and Mind-Sets." Princeton University, 2014.

Bruner, Jerome. Toward a Theory of Instruction. The Belknap Press of Harvard University Press, 1966.

Cage, John. Silence: Lectures and Writings. Wesleyan pbk. ed. Middletown, Conn: Wesleyan University Press, 1973

Cardew, Cornelius. Memories of You: Piano Solo. London: Universal Edition, 1967.

Christensen, Thomas Street. The Cambridge History of Western Music Theory. Cambridge History of Music. New York: Cambridge University Press, 2002.

Cimini, Amy. "Giles Deleuze and the Musical Spinoza." In Sounding the Virtual: Gilles Deleuze and the Theory and Philosophy of Music, edited by Brian Clarence Hulse and Nick Nesbitt, 129-44. Farnham, England, Burlington, VT: Ashgate, 2010. 
Clarke, Eric F. Ways of Listening: An Ecological Approach to the Perception of Musical Meaning. New York: Oxford University Press, 2005.

Concert: For Piano and Orchestra. n.p: Edition Peters, 1960.

Conty, Arianne. "Techno-Phenomenology: Martin Heidegger and Bruno Latour on How Phenomena Come to Presence." South African Journal Of Philosophy 32, no. 2 (2013): 211-326.

Cook, Nicholas. Beyond the Score: Music as Performance. New York: Oxford University Press, 2013.

- - - Music, Imagination, and Culture. Clarendon paperback ed. Oxford, New York: Clarendon Press, 1990.

Corbett, John. "Ephemera Underscored: Writing Around Free Improvisation." In Jazz Among the Discourses, edited by Krin Gabbard, 217-42. Durham: Duke University Press, 1995.

Craenen, Paul. Composing under the Skin: The Music-Making Body at the Composer's Desk. Leuven: Leuven Univsersity Press, 2014.

Dimpker, Christian. Extended Notation: The Depiction of the Unconventional. Wien; Zürich; Berlin; Münster: Lit, 2013.

Dolphijn, Rick, Iris van der Tuin, and OAPEN. New Materialism: Interviews and Cartographies. Open Humanitites Press [Imprint], 2012.

Driscoll, John, and Matt Rogalsky. "David Tudor's 'Rainforest': An Evolving Exploration of Resonance." Leonardo Music Journal 14 (2004): 25-30.

Eck, Cathy van. Between Air and Electricity: Microphones and Loudspeakers as Musical Instruments. New York: Bloomsbury Academic, an imprint of Bloomsbury Publishing Inc, 2017.

Email interview with Evan Parker. "Evan Parker." In The Ashgate Reserach Companion to Experimental Music, 331-36. Surrey, England and Burlingtion, VT: Ashgate, 2009.

Everett, Yayoi Uno. "Toshi Ichiyanagi and the Art of Indeterminacy I Post.” Accessed April 22, 2017. http://post.at.moma.org/content_items/90-toshi-ichiyanagi-and-the-art-of-indeterminacy.

evilpaul. Keith Rowe - Prepared Guitar. YouTube video, 2006.

Feenberg, Andrew. "Critical Theory of Technology: An Overview" 1, no. 1 (Winter 2005): 47-64.

- - - Transforming Technology: A Critical Theory Revisited.2nd ed. New York: Oxford University Press, 2002.

Feller, Ross. "Resistant Strains of Postmodernism.” In Postmodern Music/Postmodern Thought, edited by Judith Lockhead and Joseph Auner, 249-62. Studies in Contemporary Music and Culture. New York, London: Routledge, 2002.

Felski, Rita. Uses of Literature. Blackwell Manifestos. Malden, MA, Oxford: Blackwell Pub, 2008.

Ferneyhough, Brian. "Shaping Sound." In Sound, edited by Patricia Kruth and Henry Stobart, 151-72. The Darwin College Lectures, 2000.

"G O R D O N . M U M M A.” Accessed April 24, 2017. http://brainwashed.com/mumma/scores.htm.

Galliano, Luciana. "Toshi Ichiniyagi, Japanese Composer and 'Fluxus'"." Perspectives of New Music 22, no. 2 (Summer 2006): 250-56.

Gaver, William. "Technology Affordances." In Human Factors in Computing Systems Conference Proceedings, 79-84. ACM Press, 1991.

Gibson, James J. The Ecological Approach to Visual Perception. Hillsdale, N.J: Lawrence Erlbaum Associates, 1986.

Godøy, Rolf Inge, and Marc Leman. Musical Gestures: Sound, Movement, and Meaning. New York: Routledge, 2010.

Goehr, Lydia. The Imaginary Museum of Musical Works: An Essay in the Philosophy of Music. Oxford [England]: Clarendon Press, 1992.

Grant, M. J. Serial Music, Serial Aesthetics: Compositional Theory in Post-War Europe. Music in the Twentieth Century. New York: Cambridge University Press, 2001.

Grusin, Richard. "Introduction." In The Nonhuman Turn, edited by Richard Grusin, vii-xxix. Center for 21st Century Studies; 21 st Century Studies. Minneapolis, London: University of Minnesota Press, 2015.

Guest, Ann Hutchinson. Labanotation: Or, Kinetography Laban; The System of Analyzing and Recording Movement. Rev. and expanded ed. TAB Paperbook No. 27. New York: [New York] Theatre Arts Books [1973, c1970], 1970.

Haenisch, Matthias. "Materiality and Agency in Improvisation: Andrea Neumann's 'Inside Piano."” In Noise in and as Music, edited by Aaron Cassidy and Aaron Einbond, 147-70. Huddersfield: University of Huddersfield Press, 2013. 
Harding, James Martin, and John Rouse. Not the Other Avant-Garde: The Transnational Foundations of AvantGarde Performance / C Edited by James M. Harding and John Rouse. Theater--Theory/Text/Performance. Ann Arbor: The University of Michigan Press, 2006.

Hardjowirogo, Sarah-Indriyati. "Instrumentality, On the Construction of Instrumental Identity." In Musical Instruments in the 21st Century: Identities, Configurations, Practices, edited by Till Bovermann, Alberto de Campo, Hauke Egermann, Sarah-Indriyati Hardjowirogo, and Stefan Weinzierl. Singapore: Springer Nature Singapore, 2016.

Harman, Graham. "Brief SR/OOO Tutorial." Object-Oriented Philosophy, n.d. https://doctorzamalek2.wordpress.com/2010/07/23/brief-srooo-tutorial/.

- - - . Prince of Networks: Bruno Latour and Metaphysics. Anamnesis. Prahran, Vic: re.press, 2009.

- - - Tool-Being: Heidegger and the Metaphysics of Objects. Open Court: Chicago and La Salle, IL, 2002.

Harris, Tony. The Legacy of Cornelius Cardew. New York and London: Routledge, 2013.

Heath, Chris. "The New Jerry Lee Lewis Biography Is Definitively Undefinitive.” GQ, October 27, 2014. http://www.gq.com/story/the-new-jerry-lee-lewis-biography-is-definitively-undefinitive.

Hegarty, Paul. Noise/Music: A History. New York and London: Continuum, 2007.

Heidegger, Martin. Being and Time. Translated by John Macquarrie and Edward Robinson. Harper Perennial Modern Thought. New York: Harper \& Row, 1962.

- - - "The Origin of the Work of Art." In Poetry, Language, Thought, translated by Albert Hofstadter, 15-88. New York: Harper \& Row, 1971.

- - - "The Thing." In Poetry, Language, Thought, 163-86. New York: Harper \& Row, 1971.

Helgeson, Aaron. "What Is Phenomenological Music, and What Does It Have to Do With Salvatore Sciarrino?" Perspectives of New Music 51, no. 2 (Summer 2013): 4-36.

Hennion, Antoine. "The Cultural Study of Music: A Critical Introduction." In Music and Mediation: Towards a New Sociology of Music, 80-91. London: Routledge, 2003.

- - - The Passion for Music: A Sociology of Mediation. Music and Change: Ecological Perspectives; Music and Change. London, New York: Routledge, 2016.

Holmes, Thomas B. Electronic and Experimental Music: Foundations of New Music and New Listening. 2nd ed.,Revised. New York: Routledge, 2002.

Hulse, Brian Clarence. "Thinking Musical Difference: Music Theory as a Minor Science." In Sounding the Virtual: Gilles Deleuze and the Theory and Philosophy of Music, edited by Brian Clarence Hulse and Nick Nesbitt, 23-50. Farnham, England, Burlington, VT: Ashgate, 2010.

Ichiyanagi, Toshi. Music for Piano No.7. New York: C.F. Peters, 1963.

Ingarden, Roman. The Work of Music and the Problem of Its Identity. Edited by Jean G Harrell. Translated by Adam Czerniawski. Berkeley: University of California Press, 1986.

"Introduction to the PIANO PIECES," April 4, 2014. http://web.archive.org/web/20140404172922/http://www.stockhausen.org/intro_piano_pieces.html.

Ives, Charles. Piano Sonata No. 2: “Concord, Mass., 1840-1860.” New York: Associated Music, 1947.

Kanaga, David. "Wombflash Forest: Intro to Ludic Ecologonomy (Pt. 1).” Wombflash Forest, July 26, 2015. http://wombflashforest.blogspot.com/2015/04/intro-to-ludic-ecologonomy-pt-1.html.

Kartomi, Margaret J. On Concepts and Classifications of Musical Instruments. Chicago Studies in Ethnomusicology. Chicago: University of Chicago Press, 1990.

Keep, Andy. "Instrumentalizing: Approaches to Improvising with Sounding Objects in Experimental Music." In The Ashgate Research Companion to Experimental Music, edited by James Sunders, 113-30. Ashgate Research Companion. Farnham, England, Burlington, VT: Ashgate, 2009.

Kennedy, Michael, ed. Oxford Concise Dictionary of Music. Oxford, New York: Oxford University Press, 2004. Kojs, Juraj. "Notating Action-Based Music.” Leonardo Music Journal 21, no. 1 (2011): 65-72.

- - - "The Language of Action and Cyberaction-Based Music: Theory and Practice." Journal of New Music Research 38, no. 3 (2009): 285-94. doi:10.1080/09298210903141155.

Kostelanetz, Richard. Conversing With Cage. New York: Limelight Editions, 1988.

Krauss, Rosalind. "Sculpture in the Expanded Field." October 8 (Spring 1979): 30-44.

Kvifte, Tellef. "What Is a Musical Instrument?" Svensk Tidskrift För Musikforskning 90, no. 1 (2008): 45-56.

Lachenmann, Helmut. "Composing in the Shadow of Darmstadt." Translated by Richard Toop. Contemporary Music Review 23, no. 3/4 (2004): 43-53. doi:10.1080/0749446042000285663.

Latour, Bruno. "Another Way to Compose the Modern World," 1-6. Chicago, IL, 2013.

- - - . Pandora's Hope: Essays on the Reality of Science Studies. Cambridge, MA and London: Harvard University Press, 1999. 
- - - Politics of Nature: How to Bring the Sciences Into Democracy. Cambridge, Mass: Harvard University Press, 2004.

- - - Reassembling the Social: An Introduction to Actor-Network-Theory. Oxford, New York: Oxford University Press, 2005.

_ - _ "Technology Is Society Made Durable." In A Sociology of Monsters: Essays on Power, Technology and Domination, edited by J Law, 103-32. Sociological Review Monograph No. 38, 1991. http://www.brunolatour.fr/node/263.

- - - We Have Never Been Modern. Translated by Catherine Porter. Cambridge, Mass: Harvard University Press, 1993.

_ _ _. "Where Are the Missing Masses? The Sociology of a Few Mundane Artifacts." In Shaping Technology, Building Society: Studies in Sociotechnical Change, edited by Wiebe E. Bijker, 225-59. Cambridge, MA and London: The MIT Press, 1992.

- - - "Why Has Critique Run out of Steam? From Matters of Fact to Matters of Concern." Critical Inquiry 30 (Winter 2004): 225-48.

Laviolette, Patrick. "Toward a New Organology: Material Culture and the Study of Musical Instruments." Material World, November 22, 2007. http://www.materialworldblog.com/2007/11/toward-a-neworganology-material-culture-and-the-study-of-musical-instruments/.

Le Guin, Elisabeth. Boccherini's Body: An Essay in Carnal Musicology. Berkeley: University of California Press, 2006.

Leach, Neil. "Digital Tool Thinking: Object Oriented Ontology versus New Materialism," 344-51. University of Michigan Taubman College, Ann Arbor, Michigan, October 27-29. http://papers.cumincad.org/data/works/att/acadia16_344.pdf.

Leddy, Tom. "Aesthetics Today: Is Object-Oriented Ontology Good for Artists?” Aesthetics Today, August 6, 2015. http://aestheticstoday.blogspot.com/2015/08/is-object-oriented-ontology-good-for.html.

Leman, Marc. Embodied Music Cognition and Mediation Technology. Cambridge, Mass: MIT Press, 2008.

Lewis, George. "Improvised Music after 1950: Afrological and Eurological Perspectives.” In Audio Culture, edited by Christoph Cox and Daniel Warner, 272-82. New York and London: Continuum, 2004.

LIFE. Time Inc, 1948.

Lockhead, Judith. "Controlling Liberation: David Tudor and the 'Experimental' Sound Ideal." Getty Research Institute, 2001.

Lockwood, Annea. "How To Prepare a Piano." In Sound Scripts: Proceedings of the Inaugural Totally Huge New Music Festival Conference 2005, Vol. 1, 2006.

Lucier, Alvin. Action Music for Piano. Kiel: Material Press, 1967.

Mac Low, Jackson, and Anne Tardos. Thing of Beauty: New and Selected Works. Berkeley: University of California Press, 2008.

Maconie, Robin. The Works of Karlheinz Stockhausen. London, New York: Oxford University Press, 1976.

Maus, Fred Everett. "Somaesthetics of Music." Action, Criticism, and Theory for Music Education 9, no. 1 (2010): 10-25.

Merleau-Ponty, Maurice. Phenomenology of Perception. Translated by Landes David A. London, New York: Routledge, 2012.

Merriam, Alan P. The Anthropology of Music. Evanston, Ill: [Evanston, Ill.] Northwestern University Press, 1964, 1964.

Michael Evans. Piano Burning (HD). Accessed May 8, 2017. https://www.youtube.com/watch?v=WTyMmzqBjys.

Mirka, Danuta. Sonoristic Structuralism of Krzysztof Penderecki. Music Academy in Katowice, 1997.

Mol, Annemarie. "Actor-Network Theory: Sensitive Terms and Enduring Tensions." Kölner Zeitschrift Für Soziologie Und Sozialpsychologie 50, no. 1 (2010): 253-69.

Moran, Dermot. Introduction to Phenomenology. London, New York: Routledge, 2000.

Morton, Timothy. Hyperobjects. Minneapolis, MN: University of Minnesota Press, 2013.

Mumma, Gordon. Don Mills, Ont., BMI Canada [1967]. Don Mills, Ont.: BMI Canada, 1967.

_ _ _. "Gordon Mumma: MEDUIM SIZE MOGRAPH 1962." Leonardo Music Journal 21 (December 2011): $77-78$.

Nattiez, Jean-Jacques. Music and Discourse: Toward a Semiology of Music. Translated by Carolyn Abbate. Princeton, N.J: Princeton University Press, 1990.

Nercessian, Andy. The Duduk and National Identity in Armenia. Lanham, MD: Scarecrow Press, 2001. 
“Newmaterialistscartographies - New Materialism.” Accessed April 21, 2017. https://newmaterialistscartographies.wikispaces.com/New+Materialism.

Nyman, Michael. Experimental Music: Cage and Beyond. 2nd ed. Music in the 20th Century; Music in the Twentieth Century. Cambridge, U.K, New York: Cambridge University Press, 1999.

Ong, Walter J. Orality and Literacy: 30th Anniversary Edition. Routledge, 2013.

“Oral History Interview with Lee Krasner, 1964 Nov. 2-1968 Apr. 11.” Text. Accessed April 24, 2017. https:/www.aaa.si.edu/collections/interviews/oral-history-interview-lee-krasner-12507.

Orning, Tanja. "Pression - a Performance Study." Music Performance Research 5 (2012): 12-31.

Paddison, Max, and Irène Deliège. Contemporary Music: Theoretical and Philosophical Perspectives. Burlington, VT: Ashgate, 2009.

Palmer, Robert. “The Devil and Jerry Lee Lewis.” Rolling Stone, December 13, 1979. http://www.rollingstone.com/music/news/the-devil-and-jerry-lee-lewis-19791213.

Piekut, Benjamin. Experimentalism Otherwise: The New York Avant-Garde and Its Limits. California Studies in 20th-Century Music. Berkeley: University of California Press, 2011.

Revill, David. The Roaring Silence: John Cage, a Life. 1st U.S. ed. New York: Arcade Pub, 1992.

Riis, Søren. "The Symmetry Between Bruno Latour and Marin Heidegger: The Technique of Turning a Police Officer into a Speed Bump.” Social Studies of Science 38, no. 2 (April 2008).

Roell, Craig H. The Piano in America, 1890-1940. Chapel Hill: University of North Carolina Press, 1989.

Ross, Daniel. "A Summary of Bernard Stiegler, Technics and Time, 1.” Accessed April 21, 2017. https://www.academia.edu/20136235/A_Summary_of_Bernard_Stiegler_Technics_and_Time_1.

Russolo, Luigi. “U B U W E B :: Luigi Russolo - The Art of Noises.” Accessed April 17, 2017. http://www.ubu.com/papers/russolo.html.

Sachs, Curt. The History of Musical Instruments. New York: W. W. Norton \& company, inc, 1940.

Schaeffer, Pierre. In Search of a Concrete Music. Translated by Christine North and John Dack. Berkeley and Los Angeles: University of California Press, 2012.

Schmidt, Gunnar. Klavierzerstörungen in Kunst Und Popcultur. Bonn: Reimer, 2013.

- - - .Piano Activities." Piano Activities. Accessed April 24, 2017. http://piano-activities.de/englindex.html.

Schoenberg, Arnold. Theory of Harmony. Berkeley: University of California Press, 1978.

Small, Christopher. Musicking: The Meanings of Performing and Listening. Music/Culture. Hanover, NH: University Press of New England, 1998.

Steigler, Bernard. Technics and Time, 1: The Fault of Epimetheus. Translated by Richard Beardsworth and George Collins. Stanford: Stanford University Press, 1998.

Sudnow, David. Ways of the Hand: The Organization of Improvised Conduct. Cambridge, Mass: Harvard University Press, 1978.

Swithinbank, Chris. “A Structure of Physicalities: Helmut Lachenmann’s temA.” Chris Swithinbank, 14:45 200AD. http://chrisswithinbank.net/2011/03/a-structure-of-physicalities-helmut-lachenmann-tema/.

Szendy, Peter. Phantom Limbs: On Musical Bodies. First edition. New York: Fordham University Press, 2016.

Takahashi, Yuji, Sumi Adachi, and Roger Reynolds. "The Piano and Its Transformation." Perspectives of New Music 30, no. 2 (Summer 1992): 86-89.

Tarkovskiŭ, Andre ̌ Arsen'evich. Sculpting in Time: Reflections on the Cinema. New York: Alfred A. Knopf, 1987.

Théberge, Paul. Any Sound You Can Imagine: Making Music/Consuming Technology. Music/Culture. Hanover [N.H.]: Wesleyan University Press, published by University Press of New England, 1997.

Thomás, Enrique. "Musical Instruments as Scores: A Hybrid Approach.” In Second Annual Conference for Technolgoies for Music Notation and Representation. Anglia Ruskin University, Cambridge, UK: Anglia Ruskin University, 2016. http://tenor2016.tenor-conference.org/papers/16_Tomas_tenor2016.pdf.

Treatise Handbook: Including Bun No. 2, Volo Solo. London, New York: Edition Peters, 1971.

Vágnerová, Lucie. "Sirens/Cyborgs: Sound Technologies and the Musical Body." PhD dissertation, Columbia University, 2016.

Varela, Francisco J, Evan Thompson, and Eleanor Rosch. The Embodied Mind: Cognitive Science and Human Experience. Cambridge, Mass: MIT Press, 1991.

Waksman, Steve. Instruments of Desire: The Electric Guitar and the Shaping of Musical Experience. Cambridge, Mass: Havard University Press, 2000.

Wambacq, Judith. "Subject-Object in Martin Heidegger, Bruno Latour and Manuel De Landa." Constant Verlag . Accessed April 21, 2017. http://www .constantvzw.org/verlag/spip.php?article79\#. 
Warburton, Dan. Keith Rowe. Paris Transatlantic, January 2001. http://www.paristransatlantic.com/magazine/interviews/rowe.html.

Wardlaw, Matt. "Pete Townshend Recalls Negotiating with Jimi Hendrix at the Monterey Pop Festival." Ultimate Classic Rock, October 26, 2011. http://ultimateclassicrock.com/pete-townshend-jimi-hendrix-negotiatingmonterey/.

Wawzenek, Bryan. “The Day Jimi Hendrix Set His Guitar on Fire for the First Time.” Ultimate Classic Rock, March 31, 2015. http://ultimateclassicrock.com/jimi-hendrix-guitar-fire/.

Wolff, Christian, Gisela Gronemeyer, and Reinhard Oehlschlägel. Cues: Writings \& Conversations $=$ Hinweise : Schriften und Gespräche. 1. Aufl. Edition MusikTexte. Köln: MusikTexte, 1998.

Wong, Mandy Suzanne. "Sound Objects: Speculative Perspectives." PhD dissertation, University of California Los Angeles, 2012.

Žižek, Slavoj. “Objects, Objects Everywhere: A Critique of Object Oriented Ontology,” n.d. http://mariborchan.si/text/articles/slavoj-zizek/objects-objects-everywhere/. 